\title{
TU/e EmonONEN

\section{Contrast sensitivity of the human eye and its effects on image quality}

Citation for published version (APA):

Barten, P. G. J. (1999). Contrast sensitivity of the human eye and its effects on image quality. [Phd Thesis 1 (Research TU/e / Graduation TU/e), Institute for Perception Research, Eindhoven]. Technische Universiteit Eindhoven. https://doi.org/10.6100//R523072

DOI:

10.6100/IR523072

Document status and date:

Published: 01/01/1999

\section{Document Version:}

Publisher's PDF, also known as Version of Record (includes final page, issue and volume numbers)

\section{Please check the document version of this publication:}

- A submitted manuscript is the version of the article upon submission and before peer-review. There can be important differences between the submitted version and the official published version of record. People interested in the research are advised to contact the author for the final version of the publication, or visit the $\mathrm{DOI}$ to the publisher's website.

- The final author version and the galley proof are versions of the publication after peer review.

- The final published version features the final layout of the paper including the volume, issue and page numbers.

Link to publication

\section{General rights}

Copyright and moral rights for the publications made accessible in the public portal are retained by the authors and/or other copyright owners and it is a condition of accessing publications that users recognise and abide by the legal requirements associated with these rights.

- Users may download and print one copy of any publication from the public portal for the purpose of private study or research.

- You may not further distribute the material or use it for any profit-making activity or commercial gain

- You may freely distribute the URL identifying the publication in the public portal.

If the publication is distributed under the terms of Article 25fa of the Dutch Copyright Act, indicated by the "Taverne" license above, please follow below link for the End User Agreement:

www.tue.nl/taverne

Take down policy

If you believe that this document breaches copyright please contact us at:

openaccess@tue.nl

providing details and we will investigate your claim. 


\section{Contrast sensitivity of the human eye and its effects on image quality}

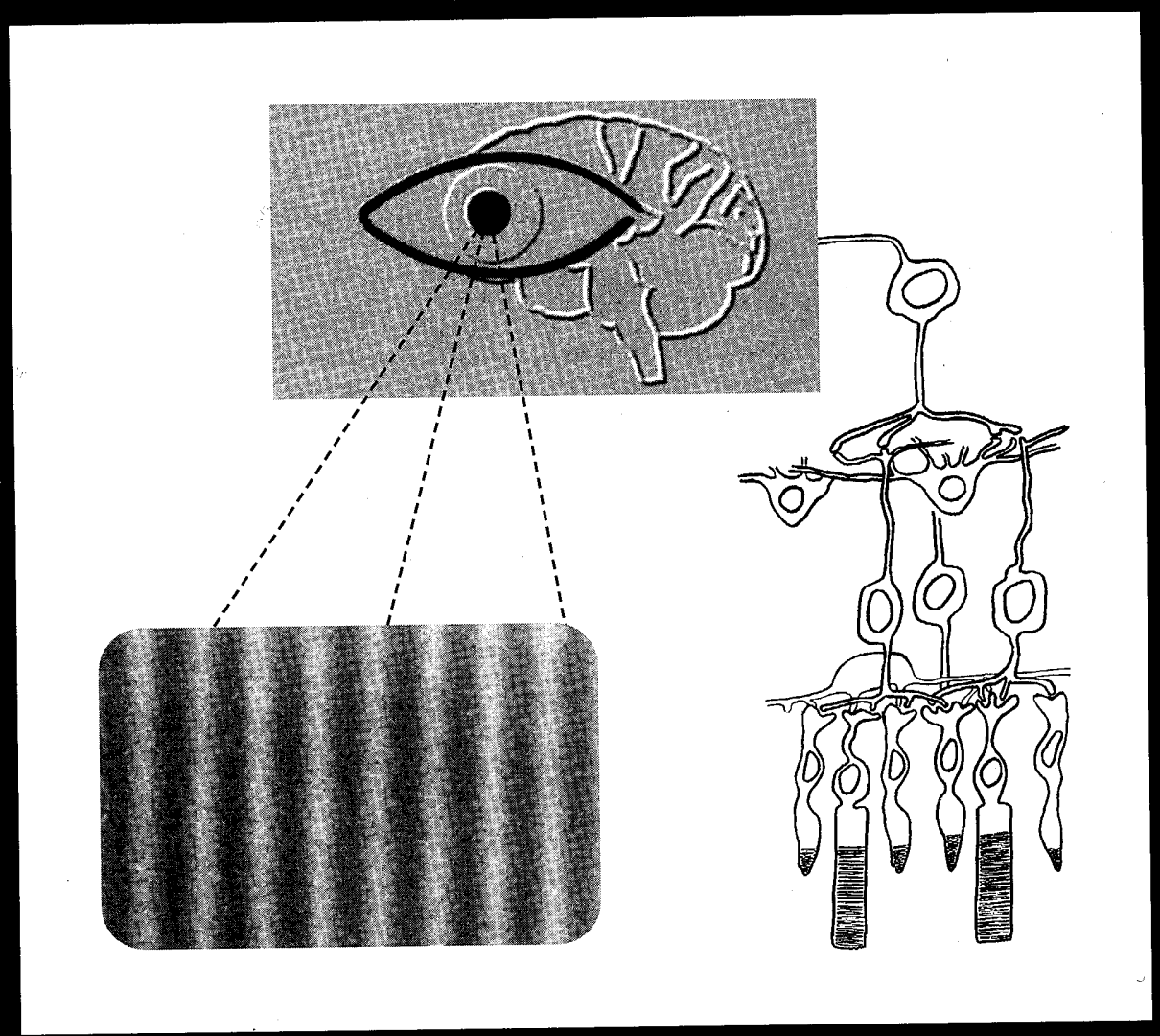

Peter G. J. Barten 


\section{Contrast sensitivity of the human eye and its effects on image quality}


Printed: All Color, Eersel

Published: HV Press, Knegsel

Copyright @ P.G.J. Barten, 1999

\section{CIP-DATA LIBRARY TECHNISCHE UNIVERSITEIT EINDHOVEN}

Barten, Peter G.J.

Contrast sensitivity of the human eye and its effects on image quality / by Peter G.J. Barten. - Knegsel : HV Press, 1999.

Proefschrift Technische Universiteit Eindhoven, 1999.

ISBN 90-9012613-9

NUGI 832

Trefwoorden: menselijk oog / visuele perceptie / contrastgevoeligheid / beeldkwaliteit Keywords: human vision / visual perception / contrast sensitivity / image quality 


\title{
Contrast sensitivity of the human eye and its effects on image quality
}

\author{
Proefschrift
}

ter verkrijging van de graad van doctor aan de Technische Universiteit Eindhoven, op gezag van de Recor Magnificus, prof.dr. M. Rem, voor een commissie aangewezen door het College voor Promoties in het openbaar te verdedigen op dinsdag 4 mei 1999 om 16.00 uur

door

Piet Gerard Joseph Barten

geboren te Amsterdam 
Dit proefschrift is goedgekeurd door de promotoren:

prof.dr.ir. J.A.J. Roufs

en

prof.dr. F.N. Hooge

Copromotor:

dr.ir. F.J.J. Blommaert 
voor Tineke,

Koen, Yvonne en Marianne 



\section{Contents}

I Introduction 1

References ................................. 5

2 Modulation threshold and noise $\quad 7$

$2.1 \quad$ Introduction . . . . . . . . . . . . . . . . . . . 7

2.2 Psychometric function $\ldots \ldots \ldots \ldots \ldots \ldots \ldots \ldots \ldots$

2.3 Basic properties of image noise $\ldots \ldots \ldots \ldots \ldots \ldots \ldots \ldots \ldots$

2.4 Effect of noise on modulation threshold $\ldots \ldots \ldots \ldots \ldots \ldots, 18$

2.5 Summary and conclusions $\ldots \ldots \ldots \ldots \ldots \ldots \ldots \ldots \ldots \ldots \ldots \ldots \ldots$

References.............................. 22

3 Model for the spatial contrast sensitivity of the eye 25

3.1 Introduction . . . . . . . . . . . . . . . . . . . . . . . 25

3.2 Outline of the model $\ldots \ldots \ldots \ldots \ldots \ldots \ldots \ldots \ldots \ldots \ldots$

3.3 Optical MTF $\ldots \ldots \ldots \ldots \ldots \ldots \ldots \ldots \ldots \ldots \ldots \ldots \ldots \ldots \ldots \ldots \ldots$

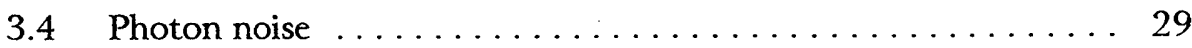

3.5 Neural noise $\ldots \ldots \ldots \ldots \ldots \ldots \ldots \ldots \ldots \ldots \ldots \ldots \ldots \ldots$

3.6 Lateral inhibition $\ldots \ldots \ldots \ldots \ldots \ldots \ldots \ldots \ldots \ldots \ldots \ldots \ldots$

3.7 Monocular versus binocular vision $\ldots \ldots \ldots \ldots \ldots \ldots \ldots . . \ldots$

3.8 Complete model . . . . . . . . . . . . . . . . . . . 36

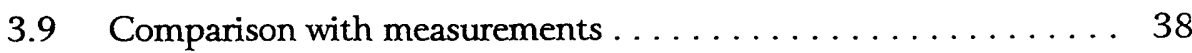

3.9.1 Measurements by DePalma and Lowry .......... . 38

3.9.2 Measurements by Patel $\ldots \ldots \ldots \ldots \ldots \ldots \ldots . \ldots \ldots$ 
3.9.3 Measurements by Robson $\ldots \ldots \ldots \ldots \ldots \ldots \ldots .40$

3.9.4 Measurements by van Nes and Bouman ......... 41

3.9.5 Measurements by Campbell and Robson .......... 44

3.9.6 Measurements by Watanabe et al. . . . . . . . . . 45

3.9.7 Measurements by Sachs et al. . . . . . . . . . . 46

3.9.8 Measurements by van Meeteren and Vos. . . . . . . . 48

3.9.9 Measurements by Howell and Hess ... . . . . . . . . 49

3.9.10 Measurements by Virsu and Rovamo .......... 50

3.9.11 Measurements by Carlson ................ 51

3.9.12 Measurements by Rovamo et al. (1992) . . . . . . . . . 52

3.9.13 Measurements by Rovamo et al. (1993a) ......... 54

3.9.14 Measurements by Rovamo et al. (1993b) ......... 55

3.9.15 Survey of the measurements. . . . . . . . . . 56

3.10 Summary and conclusions $\ldots \ldots \ldots \ldots \ldots \ldots \ldots \ldots \ldots \ldots$

Appendix A. Photon conversion factor $\ldots \ldots \ldots \ldots \ldots \ldots \ldots . \ldots \ldots$

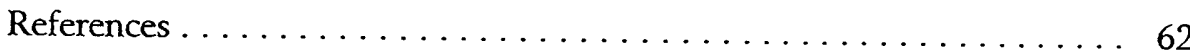

4 Extension of the contrast sensitivity model to extra-foveal vision 65

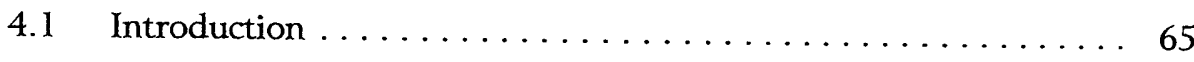

4.2 Density distribution of cones and ganglion cells ......... 66

4.2.1 Geometrical relations ................. 66

4.2 .2 Cone density distribution $\ldots \ldots \ldots \ldots \ldots \ldots \ldots 67$

4.2.3 Ganglion cell density distribution ............. 69

4.3 Effect of eccentricity on the different constants used in the model 72

4.3.1 Effect of eccentricity on resolution ............ . 72

4.3.2 Effect of eccentricity on neural noise ........... 74

4.3.3 Effect of eccentricity on lateral inhibition ........ 75

4.3.4 Effect of eccentricity on quantum efficiency ....... 76

4.3.5 Effect of eccentricity on the maximum the integration area 77 
4.4 Comparison with measurements . . . . . . . . . . 78

4.4.1 Measurements by Virsu and Rovamo . . . . . . . . . 79

4.4.2 Measurements by Robson and Graham .......... 81

4.4 .3 Measurements by Kelly. . . . . . . . . . . 83

4.4.4 Measurements by Mayer and Tyler ........... 84

4.4 .5 Measurements by Johnston. . . . . . . . . . 85

4.4.6 Measurements by Pointer and Hess ........... 86

4.4.7 Survey of the measurements $\ldots \ldots \ldots \ldots \ldots \ldots$

4.5 Summary and conclusions $\ldots \ldots \ldots \ldots \ldots \ldots \ldots$

References ........................... 90

5 Extension of the contrast sensitivity model to the temporal domain 9 I

5.1 Introduction . . . . . . . .

5.2 Generalization of the spatial contrast sensitivity model ...... 92

5.3 Temporal filter functions $\ldots \ldots \ldots \ldots \ldots \ldots \ldots$

5.4 Spatiotemporal contrast sensitivity measurements . . . . . . . . 94

5.5 Temporal contrast sensitivity measurements ........... 98

5.6 Effect of a surrounding field . . . . . . . . . . . . 100

5.7 Effect of retinal illuminance and field size on the time constants . 102

5.8 Flicker sensitivity: Ferry-Porter law . . . . . . . . . . 110

5.9 Temporal impulse response $\ldots \ldots \ldots \ldots \ldots \ldots \ldots \ldots \ldots$

5.10 Summary and conclusions $\ldots \ldots \ldots \ldots \ldots \ldots \ldots \ldots \ldots \ldots \ldots \ldots \ldots$

References ......................... 117

$6 \quad$ Effect of nonwhite spatial noise on contrast sensitivity 121

6.1 Introduction . . . . . . . . . . . . . . . . 121

6.2 Model for the masking effect of nonwhite spatial noise . . . . . 121

6.3 Measurements with narrow noise bands by Stromeyer and Julesz 124

6.4 Measurements with nonwhite noise by van Meeteren and Valeton 127

6.5 Summary and conclusions . . . . . . . . . . . . . 129 
References .............................. 130

7 Contrast discrimination model 131

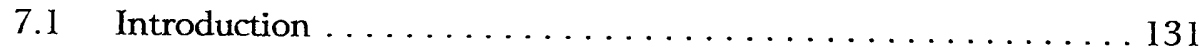

7.2 Evaluation of the psychometric function $\ldots \ldots \ldots \ldots \ldots \ldots 132$

7.3 Evaluation of the contrast discrimination model ......... 136

7.4 Comparison with contrast discrimination measurements ..... . 139

7.5 Generalized contrast discrimination model .......... 143

7.6 Summary and conclusions $\ldots \ldots \ldots \ldots \ldots \ldots \ldots \ldots \ldots$

References.............................. 147

$8 \quad$ Image quality measure $\quad 149$

$8.1 \quad$ Introduction . . . . . . . . . . . . . . . . . 149

8.2 Nonlinear effect of modulation $\ldots \ldots \ldots \ldots \ldots \ldots \ldots \ldots \ldots$

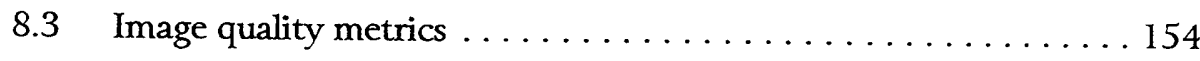

8.3.1 Modulation transfer area (MTFA) . . . . . . . . . . 155

8.3.2 Integrated contrast sensitivity (ICS) $\ldots \ldots \ldots \ldots \ldots 155$

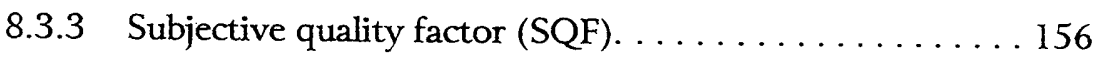

8.3.4 Discriminable difference diagram (DDD) $\ldots \ldots \ldots 156$

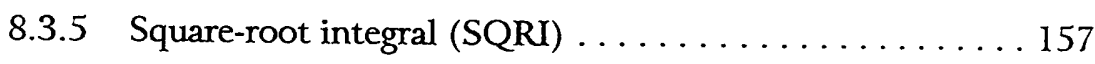

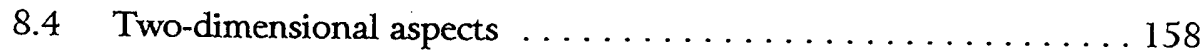

8.5 Functional analysis of image quality metrics . . . . . . . . 159

8.6 Effect of differently shaped MTFs .............. 165

8.7 Summary and conclusions $\ldots \ldots \ldots \ldots \ldots \ldots \ldots \ldots \ldots$

References.................................. 169

9 Effect of various parameters on image quality 171

$9.1 \quad$ Introduction $\ldots \ldots \ldots \ldots \ldots \ldots \ldots \ldots \ldots \ldots \ldots \ldots \ldots \ldots \ldots$

9.2 Resolution and image size $\ldots \ldots \ldots \ldots \ldots \ldots \ldots \ldots \ldots \ldots \ldots \ldots$

$9.3 \quad$ Luminance and image size $\ldots \ldots \ldots \ldots \ldots \ldots \ldots \ldots \ldots \ldots$ 
9.4 Anisotropic resolution $\ldots \ldots \ldots \ldots \ldots \ldots \ldots \ldots$

9.5 Viewing distance, display size, and number of scan lines size . . . 177

9.6 Contrast . . . . . . . . . . . . . . . . . . . . . 179

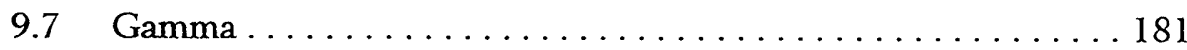

9.8 Noise .......................... 185

9.9 Pixel density and luminance quantization . . . . . . . . . . 190

9.10 Summary and conclusions ................. 193

References . . . . . . . . . . . . . . . . . . . . 194

$\begin{array}{llr}10 & \text { Epilogue } & 197\end{array}$

$\begin{array}{lr}\text { Summary } & 199\end{array}$

$\begin{array}{ll}\text { Samenvatting } & 203\end{array}$

$\begin{array}{ll}\text { Acknowledgement } & 207\end{array}$

$\begin{array}{ll}\text { Curriculum Vitae } & 209\end{array}$ 


\section{Chapter 1}

\section{Introduction}

The eye plays an important role in our life, not only for seeing objects in the surrounding world, but also for reading letters, viewing paintings, photographs, films, etc. The visual acuity of the eye is generally concerned as the most important factor for the ability of the eye for seeing objects. The acuity of the eye is usually measured by acuity tests where single black letters on a white background have to be recognized, or where the minimum visible separation is measured of black rings with a small interrupted part (Landolt rings). These tests are used for decisions about the use of certain types of eye glasses, but give no information about several other factors that also play a role in the properties of the human visual system.

Objects can generally better be distinguished from each other or from their background, if the difference in luminance or colour is large. Of these two factors, luminance plays the most important role. This study will, therefore, be concentrated on luminance, and colour will be left out of consideration. In practice, it appears that not the absolute difference in luminance is important, but the relative difference. This relative difference can be expressed by the ratio between two luminance values, which is called contrast ratio, or by the difference between two luminance values divided by the sum of them, which is simply called contrast. Both are dimensionless quantities. Objects that have only a small contrast with respect to their background are difficult to observe. The eye is more sensitive for the observation of objects, if the required amount of contrast is lower. The reciprocal of the minimum contrast required for detection is called contrast sensitivity.

For the investigation of the visual properties of the eye, different types of test patterns can be used. Generally sinusoidal test patterns are used for this purpose, as sinusoidal test patterns have an important advantage. According to Fourier analysis, the luminance pattern of an image can be considered as the sum of a number of sinusoidal luminance variations. As far as the visual system can be described by a linear system, the visibility of an image can be predicted with the aid of a Fourier analysis based on the sensitivity of the eye for sinusoidal luminance variations. Although the image forming process in the human eye is not completely linear, Fourier analysis can still be used for the area near the detection threshold, since the 
response may be assumed to be linear in that area. The use of Fourier analysis for the description of the reproduction capability of an imaging system has been introduced by Schade (1951-1955). He used it first for television systems, where the effect of cameras, signal transport and image tubes on the reproduced image can be described with the aid of Fourier analysis. Later he applied it also on the human eye as the final step in the image forming process (Schade, 1956). In this respect, also the work of Campbell \& Robson (1968) may be mentioned, who stimulated the application of Fourier analysis and the use of sinusoidal test patterns for the investigation of the visual sensitivity of the eye.

For a sinusoidal luminance pattern, contrast is defined by the amplitude of the sinusoidal variation divided by the average luminance. This quantity is called modulation depth, or shortly modulation. The minimum modulation required for the detection of this pattern is called the modulation threshold. As the contrast sensitivity is usually measured with sinusoidal luminance variations, the contrast sensitivity of the eye is generally defined as the reciprocal of the modulation threshold. The modulation threshold generally depends on the wavelength of the sinusoidal luminance variation, i.e., the distance between the maxima. The reciprocal of this wavelength is called spatial frequency. The contrast sensitivity is usually expressed as a function of this spatial frequency.

Apart from spatial luminance variations, often also temporal luminance variations occur. The contrast sensitivity of the eye for these variations can be described in the same way as for spatial luminance variations. In this case the spatial frequency has to be replaced by the temporal frequency. For the investigation of the effect of temporal luminance variations, the pioneering work by de Lange (1952, 1954) and by Kelly (1961) may be mentioned, who both applied Fourier analysis at the evaluation of temporal luminance variations.

Contrast sensitivity is sometimes measured with a periodic non-sinusoidal luminance variation. In these cases, contrast is determined by the difference between the maximum and minimum luminance divided by the sum of them. Contrast defined in this way is called Michelson contrast. For a sinusoidal luminance variation, the Michelson contrast is equal to the modulation. For a repeated non-sinusoidal luminance pattern, the equivalent sinusoidal modulation can be found by calculating the fundamental wave of this pattern with the aid of a Fourier analysis. For a square wave pattern, for instance, the modulation of the fundamental wave is $4 / \pi$ times the Michelson contrast. This has to be taken into account in the evaluation of contrast sensitivity data obtained from this type of measurements.

Knowledge of the contrast sensitivity function is important for the understanding of the visual properties of the eye. Contrary to the colorimetric sensitivity curves of the eye adopted as standard by the CIE (Commission International de l'Éclairage), 
there exists no such standard for the contrast sensitivity function of the eye. Beyond the spatial or temporal frequency of the luminance pattern, the contrast sensitivity of the eye also depends on other parameters, like luminance and field size. Defining a standard would be difficult because of the strong dependence of the contrast sensitivity on these parameters. A practical expression for the spatial contrast sensitivity function, where also these two parameters were taken into account, has been given some years ago by the author (Barten, 1990). It is an approximation formula based on contrast sensitivity measurements by van Meeteren \& Vos (1972) for a large range of luminance levels and on contrast sensitivity measurements by Carlson (1982) for a large range of field sizes. Although in this way a practical solution was found for technical applications, the formula gives no insight in the fundamental basis of the contrast sensitivity of the eye.

The main purpose of this study is to give equations for various aspects of contrast sensitivity based on fundamental assumptions about the functioning of the human eye. From these assumptions, models for the contrast sensitivity will be derived that give not only a qualitative description of contrast sensitivity but also a quantitative description. The models will be given in the form of mathematical expressions that can easily be used for practical applications. The so obtained models will extensively be compared with published measurements. The central idea of these models is the assumption that contrast sensitivity is determined by internal noise in the visual system. A part of these models was already shortly mentioned by the author in earlier publications (Barten, 1992, 1993, 1995). For practical reasons the use of the models is restricted to photopic luminance conditions. In practice, most spatial contrast sensitivity measurements are made with sinusoidal patterns in horizontal or vertical direction. In these directions, the contrast sensitivity appears to be equal. Although the contrast sensitivity of the eye can be slightly different in intermediate directions, the effect of an orientation different from the horizontal or vertical direction will be omitted, as this effect is generally very small.

In Chapter 2 first an insight will be given in the psychometric function with which the modulation threshold can be determined in a well-defined way. Based on the assumption that the contrast sensitivity is caused by internal noise, a formula will be given for the calculation of the modulation threshold from the noise. Furthermore, expressions will be given for the basic properties of image noise and for the limits of the visual system at the processing of the noise. These expressions will be used in the following chapters.

In Chapter 3 a model will be given for the spatial contrast sensitivity of the eye based on internal noise in the visual system. For this model additional assumptions will be made for the optical modulation transfer by the eye and the neural process of lateral inhibition. The model given in this chapter forms the basis of the models used in the other chapters. 
The model given in Chapter 3 is restricted to the normal condition of foveal vision where the centre of the object is imaged on the centre of the retina. Sometimes extra-foveal vision is used to observe objects that are outside the area on which the eye is concentrated. At extra-foveal vision, contrast sensitivity is reduced because of the non-homogeneity of the retina that has its optimum efficiency in the centre. In Chapter 4, the model for foveal vision given in Chapter 3 will be extended to extrafoveal vision by making some assumptions about the variation of the numerical constants used in the model with increasing eccentricity.

In Chapter 5, the model given in Chapter 3 for the spatial contrast sensitivity will be extended to the temporal domain by using some additional assumptions about the temporal behaviour of the neural elements that play a role in the transport of information. In this way a combined spatiotemporal contrast sensitivity model is obtained. With this model also flicker effects occurring at the display of television and computer images can be explained.

The contrast sensitivity can also be influenced by the presence of noise in a displayed image. Although the most common type of noise is white noise, also nonwhite noise can sometimes influence the contrast sensitivity. In Chapter 6 a generalization will be made of the expressions for white noise given in Chapter 2 . This generalization is based on the assumption of a distribution function that describes the masking of one spatial frequency by the presence of another spatial frequency.

Besides experiments with contrast detection, where a distinction has to be made between the object and a uniformly illuminated background, sometimes also experiments are made with contrast discrimination, where a distinction has to be made between two sinusoidal signals with a small difference in modulation. In Chapter 7, a model will be given for contrast discrimination. The model is based on the assumption that contrast discrimination can be considered as a special form of masking by nonwhite noise. With this model, the typical dipper shaped curves of the measurement results can be explained.

Visual aspects play an important role in the judgment of image quality. In Chapter 8 a measure will be given for the perceived quality of an image. As images largely contain modulations at suprathreshold level, not only the contrast sensitivity of the eye at threshold level is important, but also the sensitivity of the eye at higher modulation levels. Although the contrast sensitivity is defined at threshold level, it is also related to the sensitivity of the eye at higher modulation levels. In this chapter a model will be given for the nonlinear behaviour of the eye at suprathreshold levels of modulation. For this model use is made of the linear relation between perceived image quality and the number of just-noticeable differences that can be derived from the contrast discrimination model given in the previous chapter. An image quality 
measure will be given that is based on this model. This measure is called square-root integral or SQRI. This measure was already published by the author some years ago (Barten, 1990). It was only based on practical experience without the more basic information that will be given here. In this chapter also an analysis will be given of the functional suitability of various image quality measures for the description of perceived image quality.

In Chapter 9 an analysis will be given of the effect of various parameters on image quality with the aid of the image quality measure given in the previous chapter. The results will be comparted with published measurements of perceived image quality.

\section{References}

Barten, P.G.J. (1990). Evaluation of subjective image quality with the square-root integral method. Journal of the Optical Society of America A, 7, 2024-2031.

Barten, P.G.J. (1992). Physical model for the contrast sensitivity of the human eye. Human Vision, Visual Processing, and Digital Display III, Proc. SPIE, 1666, 57-72.

Barten, P.G.J. (1993). Spatio-temporal model for the contrast sensitivity of the human eye and its temporal aspects. Human Vision, Visual Processing, and Digital Display IV, Proc. SPIE, 1913, 2-14.

Barten, P.G.J. (1995). Simple model for spatial frequency masking and contrast discrimination. Human Vision, Visual Processing, and Digital Display VI, Proc. SPIE, 2411, 142-158.

Campbell, F.W. \& Robson, J.G. (1968). Application of Fourier analysis to the visibility of gratings. Journal of Physiology, 197, 551-566.

Carlson, C.R. (1982). Sine-wave threshold contrast-sensitivity function: dependence on display size. RCA Review, 43, 675-683.

Kelly, D.H. (1961). Visual responses to time-dependent stimuli. I. Amplitude sensitivity measurements. Journal of the Optical Society of America, 51, 422-429.

de Lange, H. (1952). Experiments on flicker and some calculations on an electrical analogue of the foveal systems. Physica, 18, 935-950.

de Lange, H. (1954). Relationship between critical flicker-frequency and a set of low-frequency characteristics of the eye. Journal of the Optical Society of America, 44, 380-389.

van Meeteren, A. \& Vos, J.J. (1972). Resolution and contrast sensitivity at low luminance levels. Vision Research, 12, 825-833. 
Schade, O. (1951-1955). Image gradation, graininess, and sharpness in television and motion picture systems. Journal of the SMPTE, 56, 137-171 (1951), 58, 181-222 (1952), 61, 97-164 (1953), and 64, 593-617 (1955).

Schade, O. (1956). Optical and photoelectric analog of the eye. Joumal of the Optical Society of America, 46, 721-739. 


\section{Chapter 2}

\section{Modulation threshold and noise}

\subsection{Introduction}

The modulation of a sinusoidal luminance pattern is defined by the amplitude of the sinusoidal variation divided by the average luminance. According to the generally used definition, the contrast sensitivity is the reciprocal of the modulation threshold. Therefore, the modulation threshold plays an important role in the contrast sensitivity. In this chapter the concept of the modulation threshold will be treated and the effects of noise on this threshold. In practice, it appears that there is not a fixed threshold below which a signal is not observed and above which the signal is always observed, but, instead, there is a gradual increasing probability for observing the signal. To avoid confusion, it is therefore necessary, to define which modulation level is considered as threshold. A detection probability of $50 \%$ is generally defined as the threshold and the modulation with this detection probability is defined as modulation threshold. The function that describes the detection probability as a function of signal strength is called psychometric function. This function is very useful to determine the modulation threshold in a well-defined way. The first part of this chapter will, therefore, be devoted to this function.

The psychometric function gives also a good understanding of the underlying detection mechanism. The statistical factors that influence the shape of the psychometric function may be considered to be caused by noise. This noise consists of internal noise generated in the visual system, and external noise already present in the observed image. Therefore, the basic properties of image noise will be treated in this chapter and equations will be given for the calculation of the modulation threshold from the data of the noise. The given equations will be used in the following chapters. 


\subsection{Psychometric function}

Fig. 2.1 shows an example of the psychometric function for a detection experiment by Foley and Legge (1981). In this figure the detection probability is plotted as a function of the modulation for a sinusoidal luminance pattern with a spatial frequency of 2 cycles/deg. The continuous curve drawn through the data points is a cumulative Gaussian probability function. It has already been known for more than hundred years that the psychometric function has generally the form of this function. See, for instance, Guilford (1954, pp. 3 and 126) and Le Grand (1968, pp. 237-238). After Sir Francis Galton (1885) the curve has been called Galton's ogive. The function can be described by the following expression:

$$
p(s)=\frac{1}{\sigma \sqrt{2 \pi}} \int_{-\infty}^{s} \mathrm{e}^{-\frac{\left(x-s_{0}\right)^{2}}{2 \sigma^{2}}} \mathrm{~d} x
$$

where $p$ is the detection probability, $s$ is the signal strength, $s_{0}$ is the signal strength where the detection probability is $50 \%, x$ is an integration variable, and $\sigma$ is the standard deviation of the Gaussian distribution. The minimum value of $s$ is zero. A remarkable phenomenon of this description is the fact that at a signal strength zero the probability for detection is not zero. This corresponds in practice with the wellknown situation that a signal is observed when no signal is present. This situation is

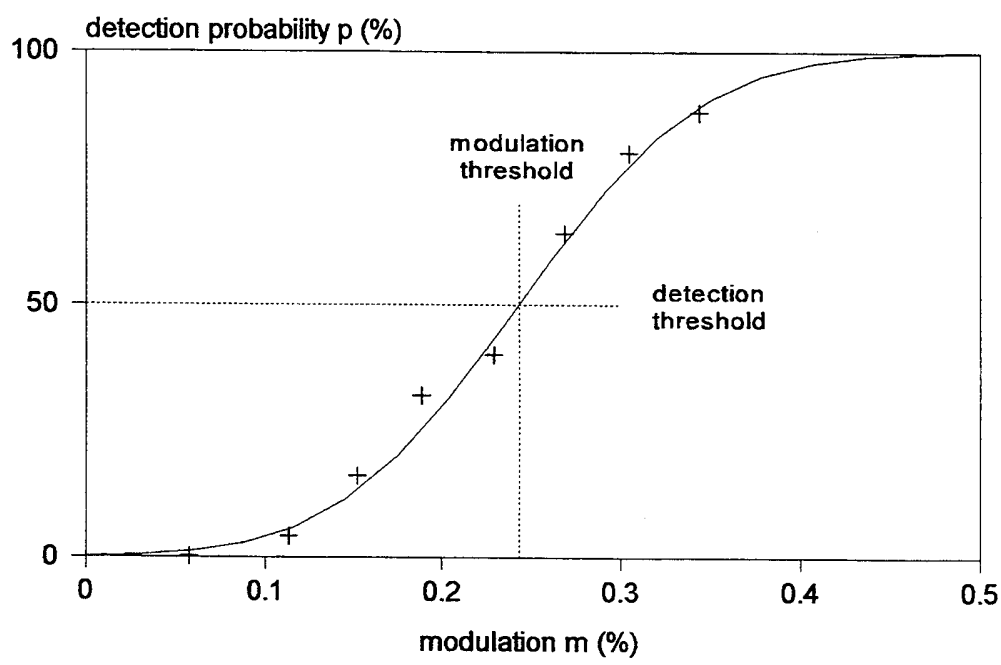

Figure 2.1: Example of a psychometric function for a sinusoidal luminance pattern with measured data from Foley \& Legge (1981). A detection probability of $50 \%$ is defined as detection threshold. The modulation with this detection probability is called modulation threshold. The continuous curve through the measured data is a cumulative probability distribution function. 


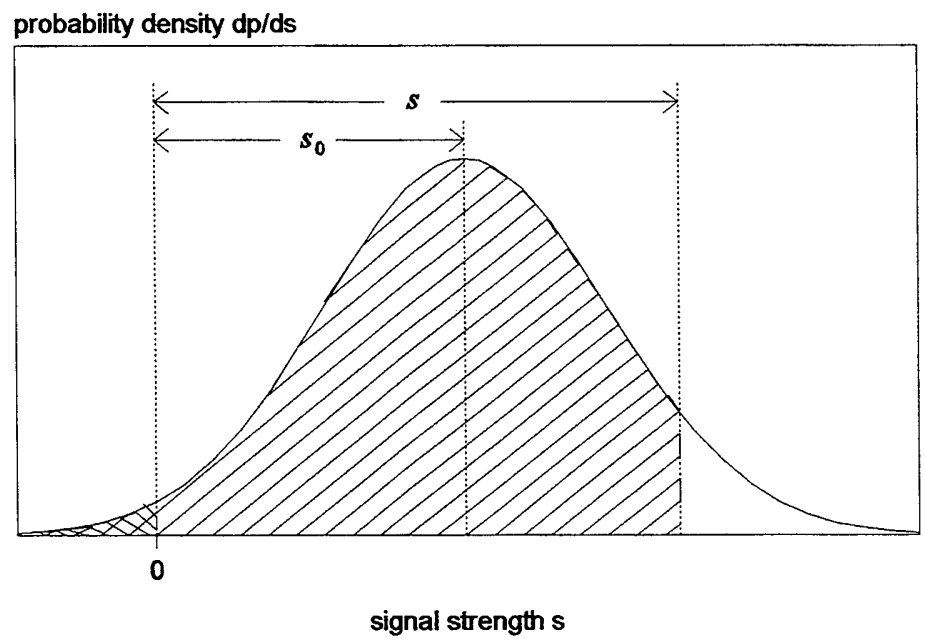

Figure 2.2: Probability density distribution for the detection of a signal with strength $s$. The shaded area indicates the detection probability $p(s)$. The double shaded area indicates the probability for false alarm $p(s=0)$.

called false alarm. Some authors use the logarithm of the signal strength as independent variable for the psychometric function. Zero signal strength then corresponds with negative infinity on the log scale. This excludes the possibility of false alarm and is therefore not in agreement with practical experience. For a sinusoidal luminance pattern, the signal strength $s$ is equal to the modulation $m$ and the signal strength $s_{0}$ is equal to the modulation threshold $m_{\mathrm{t}}$.

Fig. 2.2 shows a plot of the probability density distribution. The shaded area in this figure indicates the detection probability for a signal with strength $s$ and the double shaded area indicates the probability for false alarm. The signal strength $s_{0}$ at the maximum of the Gaussian distribution corresponds with a detection probability of $50 \%$. At this probability the slope of the psychometric function reaches a maximum. As the detection threshold is defined by a detection probability of $50 \%$, the signal strength $s_{0}$ at this probability is by definition the threshold of the signal strength. The shape of this function may be explained by assuming that the detection process is subject to statistical variations that have a Gaussian distribution. See, for instance, Thurstone (1927). These variations may be considered as internal noise. The causes of this noise will be treated in more detail in the following chapter. integral

Eq. (2.1) can also be written in the form of the well-known normal probability 
where

$$
p(z)=\frac{1}{\sqrt{2 \pi}} \int_{-\infty}^{z} \mathrm{e}^{-\frac{x^{2}}{2}} \mathrm{~d} x
$$

$$
z=\frac{s-s_{0}}{\sigma}
$$

Experimentally, the ratio between $s_{\circ}$ and $\sigma$ appears to be constant. This property is often called Crozier's law, as Crozier (1935) already found that this ratio is substantially constant over a wide range of signal conditions. Therefore, a constant $k$ can be introduced by the following expression:

$$
k=\frac{s_{0}}{\sigma}
$$

This constant may be considered as the signal-to-noise ratio required for detection. The constant $k$ has been already used by Rose (1948) to relate the luminance threshold to the external noise present in an image. Schade (1956) found that $k$ was in the range from 1.5 to 4.3 . Roufs $(1974 \mathrm{~b}, \mathrm{p}$. 875$)$ found from a large number of measurements published by several investigators, average $k$ values ranging from 2.3 to 4 . (These values were expressed by him as values of the Crozier coefficient, which is the inverse of $k$.) In practice, the $k$ can be different for different subjects and can be slightly different for different times of an experiment. It is assumed here that $k$ is about equal to 3 . This value was also used for the calculation of the curve through the data points in Fig. 2.1. The value $k=3$ may seem quite high for a signal-to-noise ratio required for detection. However, one should consider that the probability for false alarm decreases with increasing value of $k$. For $k=3$ the probability for false alarm is only $0.13 \%$. By using a high value of $k$, the visual system has probably developed during the evolution in a direction where false alarm is avoided. This goes, however, at the expense of the sensitivity for detection.

Introducing $k$ in Eq. (2.3) gives

$$
z=k\left(s / s_{0}-1\right)
$$

From this relation follows that the psychometric function can be plotted as a normalized function of $s / s_{\circ}$ with $k$ as parameter. Fig. 2.3 shows normalized plots of the psychometric function for different values of $k$. As can be seen, the steepness of the curves depends on the value of $k$. By comparing actual measurement of detection probability with these curves, the value of $k$ can easily be determined. $k$ can also be determined together with the threshold signal $s_{\circ}$ by a linear regression of $s$ with the inverse $z$ of the normal probability integral given by Eq. (2.2). In the past this regression was often made by plotting de detection probability $p$ as a function of the signal strength $s$ on probability paper. In the following some methods will be treated that are used by investigators to determine the detection threshold. They are given here for the interpretation of the measurement results that will be used in the 


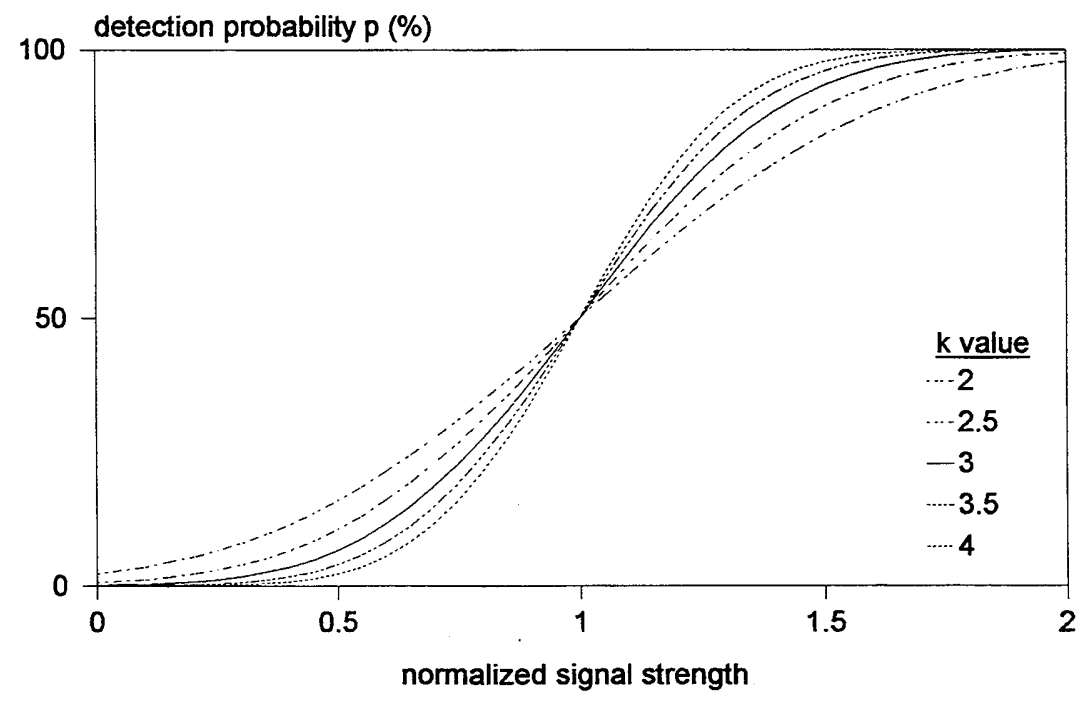

Figure 2.3: Normalized psychometric functions for different values of $k$.

following chapters.

The psychometric function is sometimes approximated by a Weibull function (Weibull, 1951). This function can be useful in the case that several processes could play a role in the detection process (Quick, 1974). The Weibull function is given by

$$
p(s)=1-\mathrm{e}^{-(\mathrm{s} / \alpha)^{\beta}}
$$

where $\alpha$ is the signal strength for a detection probability of $1-1 / e=0.632$ and $\beta$ is an exponent that determines the steepness of the psychometric function. This exponent is comparable with the constant $k$. Sometimes $\alpha$ is used as threshold signal. The signal strength $s_{0}$ where the detection probability is $50 \%$ can be calculated from $\alpha$ if $\beta$ is known:

$$
s_{0}=(\ln 2)^{1 / \beta} \alpha
$$

For the slope of the Weibull function follows from Eq. (2.6) for $s=s_{0}$

$$
\left(\frac{\mathrm{d} p}{\mathrm{~d} s}\right)_{s=s_{0}}=\frac{\ln 2}{2} \frac{\beta}{s_{0}}
$$

whereas from Eqs. (2.1) and (2.4) follows for the slope of the cumulative probability integral follows at $s=s_{0}$

$$
\left(\frac{\mathrm{d} p}{\mathrm{~d} s}\right)_{s=s_{0}}=\frac{1}{\sqrt{2 \pi}} \frac{k}{s_{0}}
$$

By making the slope of the psychometric function equal to that of the Weibull function for $s=s_{0}$ a good fit with the Weibull function can be obtained. From Eqs. 


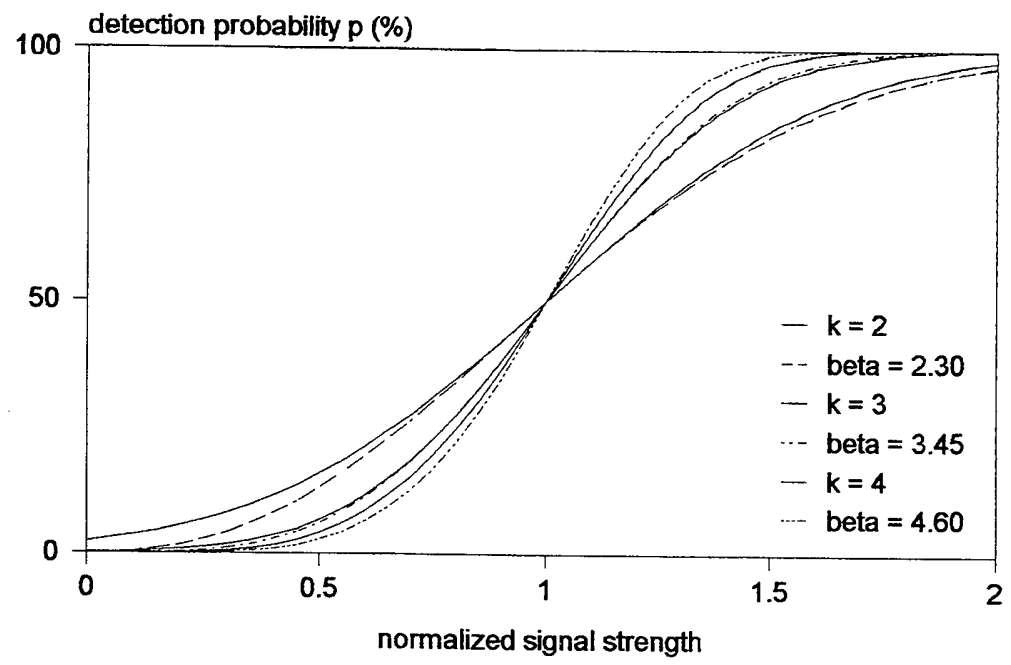

Figure 2.4: Comparison of the psychometric function for $k=2,3$, and 4 (solid curves) with the Weibull function for $\beta=2.3,3.45$, and 4.6 , respectively (dashed and dotted curves).

(2.8) and (2.9) follows that in this case

$$
k=\sqrt{\pi / 2} \ln 2 \beta=0.87 \beta
$$

This relation can be used to calculate $k$ from measured values of $\beta$. Fig. 2.4 shows a comparison of the psychometric function with the Weibull function calculated under this condition for three different values of $k$. From the figure, it can be seen that the Weibull function forms a good approximation of the psychometric function, especially for $k=3$. It can also be seen that the Weibull function excludes the possibility of false alarm.

The psychometric function is usually measured with the method of constant stimuli where each point of the function is the result of a constant number of about 100 presentations of the same signal strength. Besides the value of the detection threshold, also the value of $k$ is obtained. However, the method is very time consuming. By using some well chosen values of the signal strength around the expected threshold, the required effort can be reduced, but is still considerable. Therefore, in practice only the value of the detection threshold is measured. The most simple method to obtain this threshold is the method of adjustment, where the signal strength is varied until the signal can just be observed. This method is quicker than other possible methods, but is less reliable, because the threshold criterion is not very well defined. In measurements that will be treated in section 3.9.4 of Chapter 3, van Nes $\&$ Bouman (1967) used the method of adjustment with a lower and a higher limit for the detection. From their measurements can be derived that these limits differed 
about $12 \%$ from the average of both. This gives an indication about the accuracy that can be obtained with this method.

Another often used method is the two-alternative forced choice method or 2AFC method. The observer has to tell which one of two presented stimuli contains the test signal. If he cannot make a distinction, he has to make a guess. Taking into account that the guessing has a probability of $50 \%$ to be correct, the total probability of correct response in this experiment is given by

$$
p_{2 \mathrm{AFC}}(s)=\frac{1}{2}+\frac{1}{2} p(s)
$$

This means that a probability of $75 \%$ correct response corresponds with $50 \%$ detection probability. More generally, $x \%$ correct response corresponds with a detection probability of $2(x-50) \%$. This relation has been used to calculate the detection probability of the measurement data shown in Fig. 2.1, which were originally given as the results of a 2 AFC experiment. To arrive quickly at the situation of $75 \%$ correct response often a staircase procedure is used where the presented signal strength is changed depending on the result of foregoing observations. Sometimes staircase procedures are used that give a result corresponding with $79 \%, 84 \%$, or $90 \%$ correct response, instead of $75 \%$. This corresponds with a detection probability of $58 \%, 68 \%$, or $80 \%$ instead of $50 \%$. In such a case the obtained results must be corrected. The value of $k$ obtained from the results without correction can be described by

$$
k^{*}=\frac{s}{\sigma}
$$

where $s$ differs from the value $s_{0}$ used in Eq. (2.4). From Eq. (2.3) follows

$$
z=\frac{s-s_{0}}{\sigma}=k^{*}-k
$$

so that

$$
k=k^{*}-z
$$

The value of $z$ in this expression can be calculated by using Eq. (2.2) in inverse form. For a correct response of $79 \%, 84 \%$, and $90 \%$ in a 2 AFC experiment, the obtained $z$ value is $0.20,0.47$, and 0.84 , respectively. The value of $k$ for such an experiment has to be corrected with one of these values.

The results of a 2 AFC experiment are sometimes described with the detectability index $d^{\prime}$. The quantity $d^{\prime}$ is a measure for the detectability of a signal and is equal to the distance between the means of two distributions divided by the square root of the sum of squares of their standard deviations. It was originally designed for acoustical experiments. See Tanner \& Birdsall (1958). For a 2AFC experiment $d^{\prime}$ is defined by

$$
d^{\prime}=\sqrt{2} p_{2 \mathrm{AFC}}^{-1}(z)
$$




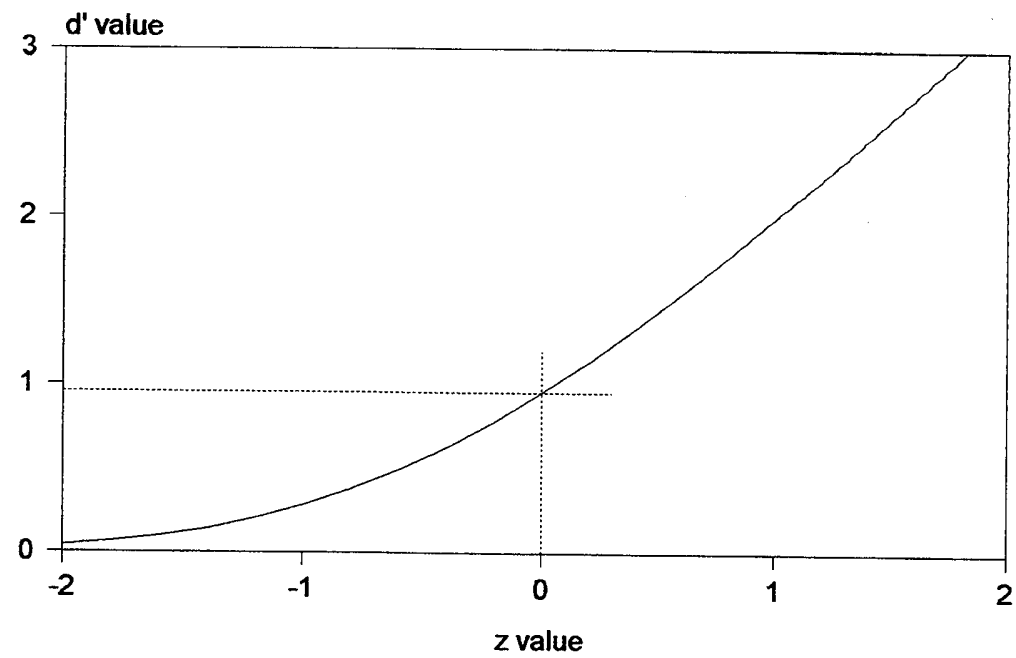

Figure 2.5: Relation between the value for $d^{\prime}$ and $z$ for a 2AFC experiment. For $p_{2 \mathrm{AFC}}=$ $75 \%, z=0$ and $d^{\prime}=0.954$.

where $p_{2 \mathrm{AFC}}{ }^{-1}(z)$ is the inverse of the normal probability integral given by Eq. (2.2), but using the probability of correct response in a 2AFC experiment, instead of the detection probability. See, for instance, Legge (1984) and Pelli (1985). According to this definition the probability of correct response is given by

$$
p_{2 \mathrm{AFC}}\left(d^{\prime}\right)=\frac{1}{\sqrt{2 \pi}} \int_{-\infty}^{\frac{d^{\prime}}{\sqrt{2}}} \mathrm{e}^{-\frac{x^{2}}{2}} \mathrm{~d} x
$$

Care should be taken in the use of $d^{\prime}$ values, because $d^{\prime}$ is not linearly related with the value of $z$ used in Eq. (2.2). The relation between $d^{\prime}$ and $z$ can be derived from Eqs. (2.16), (2.11) and (2.2) and is shown in Fig 2.5. In practice the value $d^{\prime}=1$ is used to characterize the threshold. This value corresponds with $p_{2 \mathrm{AFC}}=76 \%$ instead of $75 \%$.

For a sinusoidal luminance pattern, the signal strength $s$ is given by the modulation $m$ of the luminance pattern and the threshold signal $s_{0}$ by the modulation threshold $m_{\mathrm{t}}$ at $50 \%$ probability of detection. For a sinusoidal luminance pattern, Eq. (2.5) can be replaced by

and Eq. (2.4) by

$$
z=k\left(m / m_{t}-1\right)
$$

$$
k=\frac{m_{t}}{\sigma_{m}}
$$

where $\sigma_{\mathrm{m}}$ represents the standard deviation of the probability density distribution of 
the modulation. The value of $k$ appears not to be different from the value of $k$ for other types of signals. The probability density distribution of the modulation required for detection may be assumed to be caused by noise. It is assumed here that the standard deviation $\sigma_{\mathrm{m}}$ of this distribution is equal to the average modulation $m_{\mathrm{n}}$ of the noise wave components, where this average modulation is defined as the RMS (= root of the mean of squares) of the noise multiplied by $\sqrt{ } 2$. According to this assumption

$$
k=\frac{m_{\mathrm{t}}}{m_{\mathrm{n}}}
$$

or

$$
m_{\mathrm{t}}=k m_{\mathrm{n}}
$$

This equation forms the basis of the here given contrast sensitivity model that will be treated in the following chapters. It says that the modulation threshold is a factor $k$ larger than the average modulation of the noise wave components. The noise needs not to consist only of internal noise, but can also partly consist of external noise. In the following section will be treated how $m_{\mathrm{n}}$ can be derived from the spectral density of the noise.

\subsection{Basic properties of image noise}

According to Fourier analysis, an arbitrary luminance pattern $L(x, y, t)$ can be considered to consist of sinusoidal luminance components with spatial frequency $u$ and $v$, and temporal frequency $w$. This is also valid for the difference between the luminance $L(x, y, t)$ and the average luminance $\bar{L}$ of the pattern. In complex notation, this difference can be written as

$$
L(x, y, t)-\bar{L}=\int_{-\infty}^{+\infty} \int_{-\infty}^{+\infty} \int_{-\infty}^{+\infty} F(u, v, w) \mathrm{e}^{\mathrm{j} 2 \pi(u x+v y+w t)} \mathrm{d} u \mathrm{~d} v \mathrm{~d} w
$$

where $\mathrm{j}=\sqrt{ }-1$ and $F(u, v, w)$ is the Fourier transform of $(L(x, y, t)-\bar{L})$ given by

$$
F(u, v, w)=\int_{-\infty}^{+\infty} \int_{-\infty}^{+\infty} \int_{-\infty}^{+\infty}(L(x, y, t)-\bar{L}) \mathrm{e}^{-\mathrm{j} 2 \pi(u x+v y+w t)} \mathrm{d} x \mathrm{~d} y \mathrm{~d} t
$$

See, for instance, Bracewell (1965, p. 251). For the situations that will be considered here, the luminance pattern has limited spatial and temporal dimensions given by $X$, $Y$ and $T$, where $L$ is assumed to be zero outside this range. In this case, Eq. (2.22) can be written as

$$
F(u, v, w)=\int_{-1 / 2 X}^{+1 / 2 X} \int_{-1 / 2 Y}^{+1 / 2 Y Y} \int_{-1 / 2 T}^{+1 / 2 T}(L(x, y, t)-\bar{L}) \mathrm{e}^{-\mathrm{j} 2 \pi(u x+v y+w t)} \mathrm{d} x \mathrm{~d} y \mathrm{~d} t
$$


At the application possible truncation errors will be neglected. The average luminance of this pattern is given by

$$
\bar{L}=\frac{1}{X Y T} \int_{-1 / 2 X}^{+1 / 2 X} \int_{-1 / 2 Y}^{+1 / 2 Y} \int_{-1 / 2 T}^{+1 / 2 T} L(x, y, t) \mathrm{d} x \mathrm{~d} y \mathrm{~d} t
$$

The complex amplitude $A(u, v, w)$ of the various frequency components is given by

$$
A(u, v, w)=\frac{F(u, v, w)}{X Y T}
$$

From the last equations, it follows that for zero spatial and temporal frequencies,

$$
A(0,0,0)=0
$$

For the real amplitude $a(u, v, w)$ of the sinusoidal luminance components, it can be derived that

$$
a(u, v, w)=|A(u, v, w)|+|A(-u,-v,-w)|
$$

From Eqs. (2.23) and (2.25), it follows that

so that

$$
|A(-u,-v,-w)|=|A(u, v, w)|
$$

$$
a(u, v, w)=2|A(u, v, w)|
$$

For the applications considered here, not the amplitude of the luminance components is used, but the modulation $m(u, v, w)$, which is equal to the amplitude divided by the average luminance. So,

$$
m(u, v, w)=\frac{2|A(u, v, w)|}{\bar{L}}
$$

An important quantity of the luminance pattern is the relative variance of the luminance, defined by

$$
\sigma_{\mathrm{r}}^{2}=\frac{1}{X Y T} \int_{-1 / 2 X}^{+1 / 2 X} \int_{-1 / 2 Y}^{+1 / 2 Y} \int_{-1 / 2 T}^{+1 / 2 T} \frac{\{L(x, y, z)-\bar{L}\}^{2}}{\bar{L}^{2}} \mathrm{~d} x \mathrm{~d} y \mathrm{~d} t
$$

$\sigma_{\mathrm{r}}$ is the relative standard deviation of the luminance and is a dimensionless quantity. Applying Parseval's theorem on Eq. (2.21) gives

So,

$$
\int_{-1 / 2 X}^{+1 / 2 X} \int_{-1 / 2 Y}^{+1 / 2 Y} \int_{-1 / 2 T}^{+1 / 2 T}\{L(x, y, z)-\bar{L}\}^{2} \mathrm{~d} x \mathrm{~d} y \mathrm{~d} t=\int_{-\infty}^{+\infty} \int_{-\infty}^{+\infty} \int_{-\infty}^{+\infty}|F(u, v, w)|^{2} \mathrm{~d} u \mathrm{~d} v \mathrm{~d} w
$$

$$
\sigma_{\mathrm{r}}^{2}=\frac{1}{X Y T} \int_{-\infty}^{+\infty} \int_{-\infty}^{+\infty} \int_{-\infty}^{+\infty} \frac{|F(u, v, w)|^{2}}{\bar{L}^{2}} \mathrm{~d} u \mathrm{~d} v \mathrm{~d} w
$$

This expression shows that $\sigma_{\mathrm{r}}^{2}$ consists of the contributions of the various frequency 
components of the luminance pattern. The equation can be simplified by introducing the relative power spectral density $\Phi(u, v, w)$ defined by

$$
\Phi(u, v, w)=\frac{1}{X Y T} \frac{|F(u, v, w)|^{2}}{\bar{L}^{2}}
$$

For practical reasons this function will simply be called here spectral density, so the additions "relative" and "power" will further be omitted. By using this function, Eq. (2.33) becomes

$$
\sigma_{\mathrm{r}}^{2}=\int_{-\infty}^{+\infty} \int_{-\infty}^{+\infty} \int_{-\infty}^{+\infty} \Phi(u, v, w) \mathrm{d} u \mathrm{~d} v \mathrm{~d} w
$$

The spectral density used here is the two-sided defined spectral density (See Legge et al. 1987) contrary to the sometimes also used one-sided defined spectral density. From Eq. (2.28) follows that for a simultaneous sign change of the three frequencies

$$
\Phi(-u,-v,-w)=\Phi(u, v, w)
$$

By introducing Eq. (2.34) also in Eq. (2.30), one obtains with the aid of Eq. (2.25)

$$
m(u, v, w)=2 \sqrt{\frac{\Phi(u, v, w)}{X Y T}}
$$

For noise, the same expressions are valid as for a normal image. For noise, Eq. (2.35) can be written in the form

$$
\sigma_{\mathrm{n}}{ }^{2}=\int_{-\infty}^{+\infty} \int_{-\infty}^{+\infty} \int_{-\infty}^{+\infty} \Phi_{\mathrm{n}}(u, v, w) \mathrm{d} u \mathrm{~d} v \mathrm{~d} w
$$

where $\sigma_{n}$ is the relative standard deviation of the noise and $\Phi_{n}(u, v, w)$ is the spectral density of the noise. Since for noise, the spectral density is also the same for nonsimultaneous sign changes of the three frequencies, this expression can be simplified to

$$
\sigma_{\mathrm{n}}^{2}=\int_{0}^{\infty} \int_{0}^{\infty} \int_{0}^{\infty} \Phi_{\mathrm{n}}(u, v, w) 2 \mathrm{~d} u 2 \mathrm{~d} v 2 \mathrm{~d} w
$$

where the integration is extended only over positive frequencies.

The commonly occurring type of noise is white noise. White noise is characterized by the property that the (power) spectral density is constant over all frequencies. However, white noise is strictly only a theoretical quantity. In practical situations, white noise occurs as an approximation, valid within a given limited frequency range. Outside this range the spectral density decreases to zero. For white noise with an idealized rectangular spectrum, Eq. (2.39) becomes 


$$
\sigma_{\mathrm{n}}^{2}=\int_{u_{\min }}^{u_{\max }} \int_{v_{\min }}^{v_{\max }} \int_{w_{\min }}^{w_{\max }} \Phi_{\mathrm{n}} 2 \mathrm{~d} u 2 \mathrm{~d} v 2 \mathrm{~d} w
$$

where $u_{\min }, u_{\max }$, etc. are the minimum and maximum spatial and temporal frequencies, which are defined here as positive values. The minimum frequencies are usually nearly zero. In this case

$$
\Phi_{\mathrm{n}}=\frac{\sigma_{\mathrm{n}}{ }^{2}}{2 u_{\max } 2 v_{\max } 2 w_{\max }}
$$

whereas for nonzero minimum frequencies, $u_{\max }$ has to be replaced by $\left(u_{\max }-u_{\min }\right)$, etc. With this equation, the spectral density of the noise can be calculated when the relative standard deviation of the noise is given. If the noise is measured with samples with dimensions $\Delta x, \Delta y$ and $\Delta t$ and truncation errors are neglected, $u_{\max }=1 /(2 \Delta x)$, $v_{\max }=1 /(2 \Delta y)$, and $w_{\max }=1 /(2 \Delta t)$, as a minimum of two samples is needed to obtain one cycle. From Eq. (2.41) follows that in this case

$$
\Phi_{\mathrm{n}}=\sigma_{\mathrm{n}}^{2} \Delta x \Delta y \Delta t
$$

For the average modulation $m_{\mathrm{n}}$ of the noise wave components, one obtains with the aid of Eq. (2.37)

$$
m_{\mathrm{n}}=2 \sqrt{\frac{\Phi_{\mathrm{n}}}{X Y T}}
$$

With the aid of this equation, $m_{\mathrm{n}}$ can be calculated from the spectral density of the noise. In this equation, $X, Y$, and $T$ are the spatial and temporal size of the object covered with noise. From this relation follows that for the idealized situation of white noise, the value of $m_{\mathrm{n}}$ is equal for all frequencies.

Noise can be internal noise, present in the visual system, or external noise present in the observed image, or a combination of both. External noise can be purely static, like, for instance, grain noise in a photographic picture. Then no temporal noise is present and the factor $T$ in Eq. (2.43) and $w_{\max }$ in Eq. (2.41) have to be omitted. Sometimes the spatial noise is only present in one dimension. Then the factors $Y$ and $v_{\max }$ in these expressions have to be omitted. In these situations the spectral density has a different dimension. However, by expressing the effects of the noise in the dimensionless quantity $m_{n}$, these situations can easily be compared with each other.

\subsection{Effect of noise on modulation threshold}

The effect of external noise on the modulation threshold can also reveal much about the behaviour of the visual system with respect to internal noise. External noise can 
easily be measured, whereas these possibilities do not exist for internal noise.

Van Meeteren \& Valeton (1988) empirically found that the effect of external noise on the modulation threshold can be described by the following equation:

$$
\boldsymbol{m}_{\mathrm{t}}^{\prime}=\sqrt{\boldsymbol{m}_{\mathrm{t}}^{2}+c^{2} \boldsymbol{m}_{\mathrm{n}}{ }^{2}}
$$

where $m_{\mathrm{t}}^{\prime}$ is the modulation threshold with external noise, $m_{\mathrm{t}}$ is the modulation threshold without external noise, $m_{\mathrm{n}}$ is the average modulation of the external noise, and $c$ is a dimensionless constant. From Eq. (2.20) one would expect that the constant $c$ is equal to $k$. To verify this, investigations published by various authors have been analyzed, which consist of contrast sensitivity measurements with and without noise. These measurements were made with different types of noise: onedimensional static noise, one-dimensional dynamic noise, two-dimensional static noise, and two-dimensional dynamic noise. See Table 2.1. For these measurements $m_{\mathrm{n}}$ has been calculated with Eqs. (2.43) and (2.41) and $c$ with Eq. (2.44). The results are given in the table. In some cases the results differ considerably from the expected value 3 mentioned in section 2.2. However, during a previous study by the author (Barten, 1991), it was found that a value closer to 3 can be obtained by the introduction of some limits to the values of $X, Y$ and $T$ used in Eq. (2.43) which represent the integration area given by Eq. (2.23). These limits are caused by the limited capability of the eye to perform this integration.

For $T$, it has been assumed that with a presentation time $T_{\circ}$ of the object and an integration time $T_{\mathrm{e}}$ of the eye, the shortest of both has to be used. This may be expressed by the following expression:

$$
T=\left(\frac{1}{T_{\mathrm{o}}^{2}}+\frac{1}{T_{\mathrm{e}}^{2}}\right)^{-0.5}
$$

This expression also holds when the two are about equal. For the integration time of the eye Schade (1956) mentioned a value of $0.1 \mathrm{sec}$ for nearly all luminance levels, and only a slightly higher value at very low luminance levels. Although, values varying from $15 \mathrm{msec}$ to $300 \mathrm{msec}$ can be found in published papers for different types of conditions (Barlow, 1958; Roufs, 1974a), a constant value of $0.1 \mathrm{sec}$ will be used here under all conditions. This value may be considered as a practical average that appears to give a best fit with the data for most conditions.

In a similar way, it has been assumed that the spatial dimensions $X$ and $Y$ are limited by a maximum angular size. This limitation may be expressed by the following equations:

$$
X=\left(\frac{1}{X_{0}^{2}}+\frac{1}{X_{\max }^{2}}\right)^{-0.5}
$$

and 
Table 2.1 Values of $c$ and $k$ calculated from measurements with external noise.

\begin{tabular}{|c|c|c|c|c|c|c|c|}
\hline author & $\begin{array}{l}\text { noise } \\
\text { type }\end{array}$ & $\begin{array}{l}\text { lumin- } \\
\text { ance } \\
\left(\mathrm{cd} / \mathrm{m}^{2}\right)\end{array}$ & $\begin{array}{c}\text { object } \\
\text { size } \\
\left(\mathrm{deg}^{2}\right)\end{array}$ & $\begin{array}{c}\text { viewing } \\
\text { time } \\
\text { (sec) }\end{array}$ & $\begin{array}{l}\text { spatial } \\
\text { freq. } \\
\text { (c/deg) }\end{array}$ & $c$ & $k$ \\
\hline $\begin{array}{l}\text { Strohmeyer et al. } \\
(1972)\end{array}$ & $\begin{array}{l}1-d \\
\text { dyn. }\end{array}$ & 16 & $2.5 \times 1$ & no limit & $\begin{array}{c}1.77 \\
5 \\
10\end{array}$ & $\begin{array}{l}3.0 \\
3.2 \\
4.0\end{array}$ & $\begin{array}{l}2.9 \\
2.8 \\
2.9\end{array}$ \\
\hline Pelli (1985) & $\begin{array}{l}2-d \\
\text { dyn }\end{array}$ & 300 & $4 \times 4$ & 0.07 & 4 & $\left.4.3^{*}\right)$ & $\left.2.9^{*}\right)$ \\
\hline Thomas (1985) & $\begin{array}{l}1-d \\
\text { stat. }\end{array}$ & 65 & $3 \times 3$ & 0.5 & $\begin{array}{l}6.25 \\
8.75\end{array}$ & $\begin{array}{l}3.0 \\
4.1\end{array}$ & $\begin{array}{l}2.5 \\
3.0\end{array}$ \\
\hline Legge et al. (1987) & $\begin{array}{l}2-\mathrm{d} \\
\text { dyn. }\end{array}$ & 340 & $1 \times 1$ & 0.16 & 2 & 5.8 & 4.5 \\
\hline $\begin{array}{l}\text { van Meeteren et al. } \\
\text { (1988) }\end{array}$ & $\begin{array}{l}2-\mathrm{d} \\
\text { stat. }\end{array}$ & 100 & $1 \times 1$ & 0.2 & $\begin{array}{c}1 \\
2 \\
4 \\
9 \\
18\end{array}$ & $\begin{array}{l}3.6 \\
3.1 \\
3.1 \\
4.1 \\
4.0\end{array}$ & $\begin{array}{l}3.6 \\
3.0 \\
3.0 \\
3.5 \\
2.5\end{array}$ \\
\hline
\end{tabular}

${ }^{*}$ ) After correction of the spectral density with a factor $2^{3}$ due to the use of a different definition of the spectral noise density.

$$
Y=\left(\frac{1}{Y_{0}^{2}}+\frac{1}{Y_{\max }^{2}}\right)^{-0.5}
$$

where $X$ and $Y$ are expressed in angular size for the eye, $X_{\mathrm{o}}$ and $Y_{\mathrm{o}}$ are the angular size of the object in the $x$ and $y$ direction, respectively, and $X_{\max }$ and $Y_{\max }$ are the maximum angular dimensions of the integration area. It may further be assumed that $X_{\max }=Y_{\max }$ From measurements by Carlson (1982), which be treated in section 3.9.11 of Chapter 3, can be derived that $X_{\max }$ is about $12^{\circ}$.

However, from several published measurements (Hoekstra et al., 1974, Savoy \& McCann, 1975, Howell \& Hess, 1978, Virsu \& Rovamo, 1979, Robson \& Graham, 1981, Jamar \& Koenderink, 1983), it appears that there is also a limit of the integration area formed by a maximum number of cycles. If $N_{\max }$ is the maximum number of cycles, the maximum angular size caused by this limit is $N_{\max } u u$ where $u$ is the spatial frequency. The combined effect of this limit with the last two limits may be expressed by 


$$
X=\left(\frac{1}{X_{0}^{2}}+\frac{1}{X_{\max }^{2}}+\frac{u^{2}}{N_{\max }^{2}}\right)^{-0.5}
$$

and

$$
Y=\left(\frac{1}{Y_{0}^{2}}+\frac{1}{Y_{\max }^{2}}+\frac{u^{2}}{N_{\max }^{2}}\right)^{-0.5}
$$

These expressions replace Eqs. (2.46) and (2.47). The spatial frequency $u$ used in these expressions is the total spatial frequency, independent of orientation. As the limit formed by the maximum number of cycles is inversely proportional to spatial frequency, it has mainly effect at high spatial frequencies. In the mentioned papers, a maximum number of cycles ranging from 5 to 25 can be found. The large spread of this number is probably caused by the difference in measurement conditions. See, for instance, Estevez \& Cavonius (1975), McCann et al. (1978), McCann \& Hall (1980), van der Wildt \& Waarts (1983). A number of 15 cycles will be used here for $N_{\max }$, which gives a best fit with most of the published measurements.

The limitation of the integration area by a maximum number of cycles looks somewhat strange. However, this limitation is probably caused by the decrease of contrast sensitivity with increasing distance from the centre of the retina. This subject will be treated in more detail in section 4.4 .2 of Chapter 4 .

By using the above given expressions for $X, Y$, and $T$, corrected values of $c$ are found that are given in the last column of Table 2.1. As they may be considered to represent the actual value of $k$, they are indicated with $k$. They vary with some spread around 3 , but show a systematic dependence neither on noise type, nor on object size, luminance or spatial frequency. This means that Eq. (2.44) can be replaced by

$$
m_{\mathrm{t}}^{\prime}=\sqrt{m_{\mathrm{t}}^{2}+k^{2} m_{\mathrm{n}}^{2}}
$$

if the limitations for $X, Y$, and $T$ given by Eqs. (2.48), (2.49) and (2.45), respectively, are used for the calculation of $m_{\mathrm{n}}$.

For the often occurring situation that the object dimensions in $x$ and $y$ directions are equal, Eqs. (2.48) and (2.49) reduce to

$$
X=Y=\left(\frac{1}{X_{0}^{2}}+\frac{1}{X_{\max }^{2}}+\frac{u^{2}}{N_{\max }^{2}}\right)^{-0.5}
$$

If $X$ and $Y$ are not too much different, or if the object is circular, the same expression can be used. Then, $X_{\circ}^{2}$ has to be taken equal to the total angular area of the object.

A short time after the publication of the here given expressions for the spatial and temporal limits of the integration by the author (Barten, 1991, 1992), Rovamo et al. (1993) proposed a similar equation. However, they applied the spatial limita- 
tion only to the total area, instead of separately to the two dimensions of it. This makes no difference when the angular dimensions in $x$ and $y$ direction are nearly equal. However, when the dimensions in these directions are substantially different, the here given expressions will give a better fit with the measurements.

\subsection{Summary and conclusions}

The contrast sensitivity of the eye is defined by the modulation threshold for the detection of sinusoidal signals. In this chapter a treatment has been given of the psychometric function by which this modulation threshold can be determined in a well-defined way. Also some methods has been described that are generally used for the measurement of the modulation threshold.

It is here assumed that the modulation threshold is caused by noise. The noise consists of internal noise present in the visual system, but can partly also consist of external noise present in the observed image. According to the here given model, the modulation threshold is a fixed factor $k$ larger than the average modulation of the noise wave components. The factor $k$ is about 3.

From the basic properties of image noise, expressions have been derived for the calculation of the average modulation of the noise wave components from the data of the noise. Furthermore, equations have been given for the maximum spatial and temporal dimensions over which the eye can integrate the information of a signal. All these equations will be used in the following chapters for a further evaluation of the noise model.

\section{References}

Barlow, H.B. (1958). Temporal and spatial summation in human vision at different background intensities. Journal of Physiology, 141, 337-350.

Barten, P.G.J. (1991). Evaluation of the effect of noise on subjective image quality. Human Vision, Visual Processing, and Digital Display II, Proc. SPIE, 1453, 2-15.

Barten, P.G.J. (1992). Physical model for the contrast sensitivity of the human eye. Human Vision, Visual Processing, and Digital Display III, Proc. SPIE, 1666, 57 72.

Bracewell, R. (1965). The Fourier transform and its applications. McGraw-Hill, New York-San Francisco-Toronto-London-Sydney. 
Carlson, C.R (1982). Sine-wave threshold contrast-sensitivity function dependence on display size. RCA Review, 43, 675-683.

Crozier, W.J. (1935). On the variability of critical illumination for flicker fusion and intensity discrimination. Journal of General Physiology, 19, 503-522.

Estevez, O. \& Cavonius, C.R. (1975). Low-frequency attenuation in the detection of gratings; sorting out the artefacts. Vision Research, 16, 497-500.

Foley, J.M. \& Legge, G.E. (1981). Contrast detection and near-threshold discrimination in human vision. Vision Research, 21, 1041-1053.

Galton, F. (1885). Journal of the Anthropological Institute, 15, $205 \mathrm{ff}$.

le Grand, Y. (1968). Light, colour and vision. 2nd edition, Chapman and Hall, London.

Guilford, J.P. (1954). Psychometric methods. 2nd edition, McGraw-Hill, New York.

Hoekstra, J, van der Goot, D.P.J, van den Brink, G, and Bilsen, F.A. (1974). The influence of the number of cycles upon the visual contrast threshold for spatial sine wave patterns. Vision Research, 14, 365-368.

Howell, E.R. \& Hess, R.F. (1978). The functional area for summation to threshold for sinusoidal gratings. Vision Research, 18, 369-374.

Jamar, J.H.T. \& Koenderink, J. (1983). Sine-wave gratings scale invariance and spatial integration at suprathreshold contrast. Vision Research, 23, 805-810.

Legge, G.E. (1984). Binocular contrast summation-I. Detection and discrimination. Vision Research, 24, 373-383.

Legge, G.E., Kersten, D., and Burgess, A.E. (1987). Contrast discrimination in noise. Journal of the Optical Society of America A, 4, 391-404.

McCann, J.J. \& Hall, J.A. Jr (1980). Effects of average-luminance surrounds on the visibility of sine-wave gratings. Joumal of the Optical Society of America, 70, 212219.

McCann, J.J., Savoy R.L., and Hall, J.A. Jr (1978). Visibility of low-frequency sinewave targets dependence on number of cycles and surround parameters. Vision Research, 18, 891-894.

van Meeteren, A \& Valeton, J. (1988). Effects of pictorial noise interfering with visual detection. Journal of the Optical Society of America A, 5, 438-444.

Nachmias, J. \& Sansbury, R.V. (1974). Grating contrast discrimination may be better than detection. Vision Research, 14, 1039-1041.

van Nes, F.L. \& Bouman, M.A. (1967). Spatial modulation transfer in the human eye. Journal of the Optical Society of America, 57, 401-406.

Pelli, D.G. (1985). Uncertainty explains many aspects of visual contrast detection and discrimination. Journal of the Optical Society of America A, 2, 1508-1532.

Quick, R.F. (1974). A vector magnitude model of contrast detection. Kybernetik, 16, 
65-67.

Robson, J.G. \& Graham, N. (1981). Probability summation and regional variation in contrast sensitivity across the visual field. Vision Research, 21, 409-418.

Rose, A (1948). The sensitivity performance of the human eye on an absolute scale. Journal of the Optical Society of America, 38, 196-208.

Roufs, J.A.J. (1974a). Dynamic properties of vision-IV. Thresholds of decremental flashes, incremental flashes and doublets in relation to flicker fusion. Vision Research, 14, 831-851.

Roufs, J.A.J. (1974b). Dynamic properties of vision-VI. Stochastic threshold fluctuations and their effect on flash-to-flick er sensitivity ratio. Vision Research, 14, $871-888$.

Rovamo, J., Luntinen, O., and Näsänen, R. (1993). Modelling the dependence of contrast sensitivity on grating area and spatial frequency. Vision Research, 33, 2773-2788.

Savoy, R.L. \& McCann, J.J. (1975). Visibility of low-spatial-frequency sine-wave targets dependence on the number of cycles. Journal of the Optical Society of America, 65, 343-350.

Schade, O. (1956). Optical and photoelectric analog of the eye. Journal of the Optical Society of America, 46, 721-739.

Stromeyer, C.F. \& Julesz, B. (1972). Spatial frequency masking in vision: critical bands and spread of masking. Journal of the Optical Society of America, 62, 1221 1232.

Tanner, W.P. \& Birdsall, T.G. (1958). Definitions of $d^{\prime}$ and $\eta$ as psychophysical measures. Journal of the Acoustical Society of America, 30, 922-928.

Thomas, J.P. (1985). Effect of static-noise and grating masks on detection and identification of grating targets. Journal of the Optical Society of America A, 2, 1586-1592.

Thurstone, L.L. (1927). Psychophysical analysis. American Journal of Psychology, 38, 368-389.

Virsu, V. \& Rovamo, J. (1979). Visual resolution, contrast sensitivity, and the cortical magnification factor. Experimental Brain Research, 37, 475-494.

Weibull, W. (1951) A statistical distribution function of wide applicability. Journal of Applied Mechanics, 18, 292-297.

van der Wildt, G.J. \& Waarts, R.G. (1983). Contrast detection and its dependence on the presence of edges and lines in the stimulus field. Vision Research, 23, 82 l -830 . 


\section{Chapter 3}

\section{Model for the spatial contrast sensitivity of the eye}

\subsection{Introduction}

In the previous chapter equations have been given for the effect of noise on contrast sensitivity. In this chapter these equations will be used for a model of the spatial contrast sensitivity of the eye. This model is based on the assumption that the contrast sensitivity is mainly determined by the internal noise of the visual system. For this model additional assumptions have to be made about the optical properties of the eye and about the neural processing of the information by lateral inhibition. In this way a quantitative description of the contrast sensitivity function is obtained that also explains the dependence of the contrast sensitivity on luminance and field size. The predictions by this model will be compared with a large number of published measurements of the contrast sensitivity. For practical reasons the application of the model is restricted to photopic vision.

In the model use will be made of the modulation transfer function or MTF. This function describes the filtering of the modulation by an image forming system as a function of the spatial frequency. The use of an MTF has the advantage that the MTFs of different parts of an image forming system can simply be multiplied with each other to obtain the total effect on the final image. The MTF is based on the application of Fourier analysis and can, therefore, only be applied to linear systems. However, as the model is based on threshold signals and signals may be assumed to be linear in that area, nonlinearity effects may be neglected. From a comparison of the model with measured data, it appears that this neglect is justified.

\subsection{Outline of the model}

In the model, it is assumed, that a luminance signal that enters the eye is first filtered by the optical MTF of the eye and then by the MTF of a lateral inhibition process. It 
is further assumed that the optical MTF is mainly determined by physical processes taking place in the eye lens and the retina, and that the MTF of the lateral inhibition process is determined by neural processing. For a comparison of the signal with the internal noise Eq. (2.20) in Chapter 2 has to be modified into

$$
m_{\mathrm{t}} M_{\text {opt }}(u) M_{\mathrm{lat}}(u)=k m_{\mathrm{n}}
$$

where $M_{\text {opt }}(u)$ is the optical MTF of the eye, $M_{\text {lat }}(u)$ is the MTF of the lateral inhibition process and $m_{\mathrm{n}}$ is the average modulation of the internal noise. After applying Eq. (2.43) to $m_{\mathrm{n}}$ one obtains

$$
m_{\mathrm{t}} M_{\mathrm{opt}}(u) M_{\mathrm{lat}}(u)=2 k \sqrt{\frac{\Phi_{\mathrm{n}}}{X Y T}}
$$

where $\Phi_{\mathrm{n}}$ is the spectral density of the internal noise and $X, Y$, and $T$ are given by Eqs. (2.48), (2.49), and (2.45), respectively, that describe the spatial and temporal dimensions of the observed object, taking into account the limited integration area of the visual system.

Internal noise is partly due to photon noise caused by statistical fluctuations of the number of photons that generate an excitation of the photo-receptors, and partly due to neural noise caused by statistical fluctuations in the signal transport to the brain. Although the original image contains already photon noise before entering the eye, photon noise is not considered here as external noise, but as internal noise. This treatment might be clear from the fact that the high spatial frequency components of this noise are not filtered by the lowpass filter formed by the optical MTF at the entrance of the eye. The spectral density of the internal noise may, therefore, be written in the form

$$
\Phi_{\mathrm{n}}=\Phi_{\mathrm{ph}} M_{\mathrm{lat}}^{2}(u)+\Phi_{0}
$$

where $\Phi_{\mathrm{ph}}$ is the spectral density of the photon noise, and $\Phi_{0}$ is the spectral density of the neural noise. In this expression, it is assumed that the photon noise is filtered together with the signal by the lateral inhibition process.

Fig. 3.1 shows a block diagram of the model. For completeness, also external noise consisting of display noise is mentioned in this figure. Display noise occurs, for instance, at the observation of a television image with noise or at the observation of a photograph with grain noise. The spectral noise density of this external noise adds to the spectral noise density of the internal noise after multiplication by $M_{\text {opt }}{ }^{2}(u) M_{\text {lat }}{ }^{2}(u)$. However, in most of the cases treated in this chapter no external noise is present.

Insertion of Eq. (3.3) in Eq. (3.2) gives

$$
m_{\mathrm{t}} M_{\mathrm{opt}}(u) M_{\mathrm{lat}}(u)=2 k \sqrt{\frac{\Phi_{\mathrm{ph}} M_{\mathrm{lat}}{ }^{2}(u)+\Phi_{0}}{X Y T}}
$$




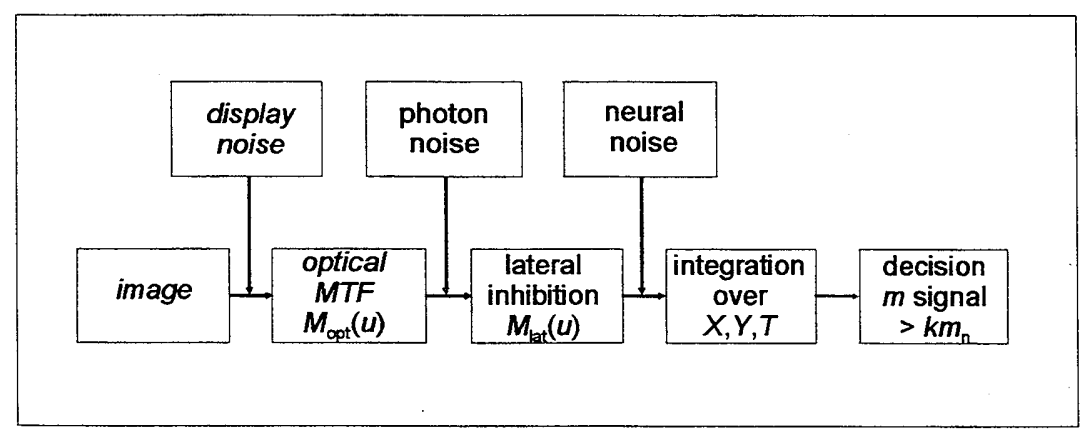

Figure 3.1: Block diagram of the processing of information and noise in the human visual system according to the here given spatial contrast sensitivity model.

For the contrast sensitivity, which is the inverse of the modulation threshold, follows from this relation

$$
S(u)=\frac{1}{m_{\mathrm{t}}}=\frac{M_{\mathrm{opt}}(u)}{2 k} \sqrt{\frac{X Y T}{\Phi_{\mathrm{ph}}+\Phi_{0} / M_{\mathrm{lat}}^{2}(u)}}
$$

This expression forms the basis of the here given contrast sensitivity model. The various components of this expression will be treated in more detail in the following sections.

\subsection{Optical MTF}

The optical MTF used in the model does not include only the optical MTF of the eye lens, but also the effects of stray light in the ocular media, diffusion in the retina and the discrete structure of the photo-receptors. These effects have to be convolved with each other to obtain the total effect. For many convolutions the central limit theorem may be applied. This theorem says that the total effect of several lowpass MTFs can be described by a Gaussian function. See, for instance Bracewell (1965, pp. 168-173). Therefore, it is assumed here that the optical MTF of the eye can be described by the following Gaussian function:

$$
M_{\text {opt }}(u)=\mathrm{e}^{-2 \pi^{2} \sigma^{2} u^{2}}
$$

where $\sigma$ is the standard deviation of the line-spread function resulting from the convolution of the different elements of the convolution process. That this function forms a good approximation of the optical MTF of the eye, appears from a comparison of the high frequency behaviour of the model with measured data of the contrast 
sensitivity function.

The quantity $\sigma$ in Eq. (3.6) generally depends on the pupil diameter $d$ of the eye lens. For very small pupil diameters, $\sigma$ increases inversely proportionally with pupil size because of diffraction, and for large pupil diameters, $\sigma$ increases about linearly with pupil size because of chromatic aberration and other aberrations. See Vos et al. (1976, Fig. 3). As according to these authors, diffraction effects become noticeable only at pupil diameters smaller than $2 \mathrm{~mm}$, they may be neglected under normal viewing conditions. Therefore, it is assumed here that the dependence on pupil size can simply be expressed by the following equation:

$$
\sigma=\sqrt{\sigma_{0}^{2}+\left(C_{\mathrm{ab}} d\right)^{2}}
$$

where $\sigma_{0}$ is a constant, $C_{\mathrm{ab}}$ is a dimension constant that describes the increase of $\sigma$ at increasing pupil size and $d$ is the diameter of the pupil. From an evaluation of contrast sensitivity measurements, it appears that for observers with a good vision, $\sigma_{0}$ is about $0.5 \mathrm{arcmin}$ and $C_{\mathrm{ab}}$ is about $0.08 \mathrm{arc} \mathrm{min} / \mathrm{mm}$. The value of $\sigma_{0}$ is only partly determined by the optical effect of the eye lens. It is also determined by the density of the photo-receptors. As the density of the photo-receptors decreases with increasing distance from the centre of the retina, $\sigma_{0}$ increases with this distance. See Chapter 4. However, for the normal situation of foveal vision treated in this chapter $\sigma_{0}$ may be considered as constant.

The diameter $d$ of the pupil generally depends on the average luminance of the observed object. To calculate the pupil size for a given luminance, the following simple approximation formula given by Le Grand (1969, p. 99) can be used:

$$
d=5-3 \tanh (0.4 \log L)
$$

where $d$ is the pupil diameter in $\mathrm{mm}$ and $L$ is the average luminance in $\mathrm{cd} / \mathrm{m}^{2}$. This expression is similar to other formulae earlier given by Crawford (1936), Moon \& Spencer (1944) and De Groot \& Gebhard (1952). These formulae represent an average of various measurement data that show a large spread. Apart from the difference between different observers, this spread is also caused by the difference in the angular size of the object fields used in the experiments. Bouma (1965) investigated the effect of different field sizes. From his measurements an approximately quadratic dependence on field size can be derived. By assuming that Eq. (3.8) is valid for an average field size of $40^{\circ} \times 40^{\circ}$, one obtains the following approximation formula where also the field size is taken into account

$$
d=5-3 \tanh \left\{0.4 \log \left(L X_{0}^{2} / 40^{2}\right)\right\}
$$

where $X_{\circ}$ is the angular field size of the object in degrees. For a rectangular field $X_{\circ}^{2}$ has to be replaced by $X_{\circ} Y_{\circ}$, and for a circular field $X_{\circ}^{2}$ has to be replaced by $\pi / 4 \times D^{2}$ where $D$ is the field diameter in degrees. This expression will generally be used here as a refinement of Eq. (3.8). It is in fact only valid for young adult observers. At older ages, the pupil size decreases with age. See, for instance, Kumninck (1954, Fig. 4) 
and Bouma (1965, Fig. 7.30).

\subsection{Photon noise}

The effect of photon noise on the contrast sensitivity of the eye was first discovered by de Vries (1943) and was later evaluated by Rose (1948) who explicitly cites the paper of de Vries. Often an earlier paper of Rose (1942) is cited for this effect, but this paper does not contain any mentioning of this effect.

According to de Vries the detection threshold at low luminance levels is determined by fluctuations in the number of photons that cause an excitation of the photo-receptors. Let the number of these photons within an area $\Delta x \Delta y$ and time $\Delta t$ be $n$. For the statistical process of the arbitrary arriving photons, the standard deviation of this number is equal to $\sqrt{n} \bar{n}$ where $\bar{n}$ is the average value of $n$. This average value may be expressed in the average flux density $j$ of the photons with the equation

$$
\bar{n}=j \Delta x \Delta y \Delta t
$$

For the relative standard deviation $\sigma_{n}$ of $n$ holds

$$
\sigma_{n}=\frac{\sqrt{\bar{n}}}{\bar{n}}=\frac{1}{\sqrt{j \Delta x \Delta y \Delta t}}
$$

According to de Vries these fluctuations form the background noise that hampers the observation of an object. Application of Eq. (2.42) gives for the spectral density of the photon noise

$$
\Phi_{\mathrm{ph}}=\sigma_{n}^{2} \Delta x \Delta y \Delta t
$$

where $\sigma_{n}$ has replaced $\sigma_{n}$ and $\phi_{\text {ph }}$ has replaced $\phi_{n}$. Inserting Eq. (3.11) in this expression gives

$$
\Phi_{\mathrm{ph}}=\frac{1}{j}
$$

This equation says that the spectral density of photon noise is equal to the inverse of the average flux density of the photons on the retina that cause an excitation of the photo-receptors. The flux density on the retina can be derived from the luminous intensity of the light entering the eye with the following expression:

$$
j=\eta p E
$$

where $\eta$ is the quantum efficiency of the eye, $p$ is the photon conversion factor for the conversion of light units in units for the flux density of the photons and $E$ is a luminance quantity proportional to the retinal illuminance. Each of these quantities will be treated in more detail in this section.

The quantum efficiency $\eta$ is defined here as the average number of photons 
causing an excitation of the photo-receptors, divided by the number of photons entering the eye. Although the quantum efficiency varies in principle with the wavelength, the wavelength dependence will be taken into account in the photon conversion factor. See also Appendix A of this chapter. In this way $\eta$ represents the quantum efficiency at the maximum of the spectral sensitivity curve. Contrary to what one would expect, the quantum efficiency of the eye is very low. From an evaluation of contrast sensitivity measurements, it appears that $\eta$ is about $3 \%$ or less (See, for instance, Table 3.1 in section 3.9.15). Van Meeteren (1978) found even values of $2 \%$ and less by measuring the contrast sensitivity with and without artificial image intensification. He tried to explain the low quantum efficiency by various causes of losses. A part of the light is lost by absorption in the ocular media, another part falls in the interstices between the photo-receptors, a part of the light falling on a photo-receptor is not absorbed, and finally not every absorbed photon causes excitation. However, van Meeteren could not explain the low quantum efficiency that he measured by an estimate of these losses. An explanation could maybe found in fluctuations of the excitation of the photo-receptors. If these fluctuations are not negligible, they form an additional noise source, which could be translated in effectively lower quantum efficiency.

The photon conversion factor $p$ in Eq. (3.14) is defined as the number of photons per unit of time, per unit of angular area, and per unit of luminous flux per angular area of the light entering the eye. Absorption losses and other losses are already taken into account in the quantum efficiency $\eta$. The number of photons generally depends on the spectral wave length of the light. Equations for the calculation of the photon conversion factor from the spectral composition of the light source are given in Appendix $\mathrm{A}$ of this chapter. They are derived from basic photometric and physical quantities. For the calculation of the photon conversion factor a distinction has to be made between photopic vision (= daylight vision), and scotopic vision (= night vision). At photopic vision the cones act as photo-receptor, whereas at scotopic vision the rods act as photo-receptor. The spectral sensitivity for photopic vision is different from that for scotopic vision, as the cones are less sensitive for blue light and the rods are less sensitive for red light. In Table 3.2 of Appendix A of this chapter, numerical values of the photon conversion factor are given for different light sources. Although the use of the here given contrast sensitivity model is restricted to photopic viewing, also data for scotopic viewing are given as general information.

The quantity $E$ in Eq. (3.14) is proportional to the retinal illuminance and can be calculated from the luminance $L$ of the object and the pupil size $d$ with the following equation

$$
E=\frac{\pi d^{2}}{4} L
$$

If the pupil size is expressed in $\mathrm{mm}$ and the luminance in $\mathrm{cd} / \mathrm{m}^{2}, E$ is given in 
Trolands. 1 Troland corresponds with a retinal illuminance of $2 \times 10^{-3}$ lux, taking into account the absorption of the light in the ocular media and the angular area of the pupil seen from the retina. Although the Troland has not the dimension of illuminance, it is for practical reasons chosen as a measure of retinal illuminance. The transition between scotopic and photopic vision occurs at a level between 1 and 10 Troland. The pupil size can be measured, or can be derived from the luminance with Eq. (3.9).

For the photopic viewing conditions used here, Eq. (3.15) has to be corrected for the Stiles-Crawford effect. For light falling on the cones Stiles \& Crawford (1933) found that rays entering near the edge of the pupil are visually much less effective than rays near the center of the pupil. From the work by Stiles and Crawford, Moon $\&$ Spencer (1944) and Jacobs (1944) derived an expression that forms a modification of Eq. (3.15) and may be written in the following form:

$$
E=\frac{\pi d^{2}}{4} L\left\{1-(d / 9.7)^{2}+(d / 12.4)^{4}\right\}
$$

where $d$ is expressed in mm. This expression will be used in the model. Although the decrease of the quantum efficiency by the Stiles-Crawford effect could also have been taken into account in the quantum efficiency $\eta$, the use of this expression is preferred here for practical reasons. For large pupil sizes, the correction for the Stiles-Crawford effect can amount to $50 \%$.

By combining Eqs. (3.13) and (3.14) one obtains

$$
\Phi_{\mathrm{ph}}=\frac{1}{\eta p E}
$$

According to this equation and Eq. (3.5), contrast sensitivity increases at low luminance levels with the square root of retinal illuminance. At these levels the effect of photon noise is so large that the effect of neural noise may be neglected. This square root behaviour is known as de Vries-Rose law. An example of this behaviour will be shown by the measurement data given in Fig. 3.21 of section 3.9.13.

\subsection{Neural noise}

In the model, it is assumed that neural noise is caused by statistical fluctuations in the signal transported to the brain. Contrary to electronic image systems, where usually only one wire is used for the transport of a signal, the image formed on the retina of the eye is transported to the brain by many fibres in parallel. When the image consists of a uniformly illuminated field, one may not expect that the different parts of this field will be reproduced by all nerve fibres in the same amount. Small differences between the different fibres will cause noise in the image arriving in the brain. The size of these differences can be estimated from the spectral density of the 
noise. From a comparison of contrast sensitivity measurements with the results obtained with the model, the spectral density $\Phi_{0}$ of the neural noise may be estimated to be about $0.03 \times 10^{-6} \mathrm{sec} \mathrm{deg}^{2}$ (See, for instance, the measurements shown in sections 3.9.11 and 3.9.12). From Eq. (2.42) follows for the relative standard deviation of the signal transported by an individual nerve fibre:

$$
\sigma=\sqrt{\frac{\Phi_{0}}{\Delta x \Delta y \Delta t}}
$$

where $\Delta x \Delta y$ is the retinal angular area covered by one nerve fibre, and $\Delta t$ is the integration time of the visual system. The density of ganglion cells from which the nerve fibers originate may be estimated to be about 1,800 cells per $\mathrm{deg}^{2}$ in the centre of the retina (See section 4.2 of Chapter 4 ). This means that $l /(\Delta x \Delta y) \approx 1,800 / \mathrm{deg}^{2}$. If for the integration time of the eye a value of $0.1 \mathrm{sec}$ is used, the relative standard deviation of the signal transported by the individual nerve fibres becomes

$$
\sigma=\sqrt{\frac{0.03 \cdot 10^{-6} \cdot 1,800}{0.1}}=0.023
$$

This is a fluctuation of $2.3 \%$, which may be considered as a reasonable value.

In the model, it is assumed that neural noise does not depend on retinal illuminance. At high retinal illuminance levels where the effect of photon noise decreases, neural noise remains as only noise source. According to Eq. (3.5) contrast sensitivity becomes then independent of luminance. This behaviour is known as Weber's law. An example of this behaviour will be shown by the measurement data given in Fig. 3.21 of section 3.9.13.

\subsection{Lateral inhibition}

In the here given model, it is assumed that the luminance signal and the added photon noise are filtered in the neural system by a lateral inhibition process that attenuates low spatial frequency components. Since the contrast sensitivity appears to decrease linearly with the inverse of spatial frequency at low spatial frequencies, the effect of lateral inhibition can be characterized by an MTF that increases linearly with spatial frequency at low spatial frequencies up to $l$ at a certain spatial frequency and remains further constant at higher spatial frequencies. From an investigation of natural scenes, Field (1987) found that the amplitude of the spatial frequency components of natural images decreases linearly with spatial frequency. This property of natural scenes is obviously compensated at low spatial frequencies by the increase of the MTF in this area due to the lateral inhibition. The existence of lateral inhibition may, therefore, probably be explained by the fact that the eye can make in this way a more efficient use of the dynamic range of signals that it can handle. 
As was already supposed by Schade (1956) and was experimentally confirmed by Enroth-Cugell and Robson (1966) at their investigation with cats, lateral inhibition consists of the subtraction of a spatially lowpass filtered signal from a signal that is directly collected from the photo-receptors. Enroth-Cugell and Robson described the point-spread function of this process by a difference of two Gaussian functions, which has the form of a Mexican hat. In publications this model is called DOG model (difference of Gaussians). However, it leads to a quadratic increase of contrast sensitivity at low spatial frequencies, whereas measurements of the contrast sensitivity clearly show a linear increase. Therefore, a different approach will be followed here.

From an evaluation of published contrast sensitivity measurements, it was found that the MTF of the lateral inhibition process may be described by the following approximation formula (Barten, 1992):

$$
M_{\text {lat }}(u)=\sqrt{1-\mathrm{e}^{-\left(\mathrm{u} / \mathrm{u}_{0}\right)^{2}}}
$$

This function is shown by the solid curve in Fig. 3.2. It gives the required linear increase of the MTF with spatial frequency up to the value $I$ at a spatial frequency $u_{0}$ above which the lateral inhibition ceases. From a best fit of the model with the published contrast sensitivity measurements given in section 3.9 , it appears that $u_{0}$ is about 7 cycles/deg. It is further assumed that the lateral inhibition process is rotational ly symmetric.

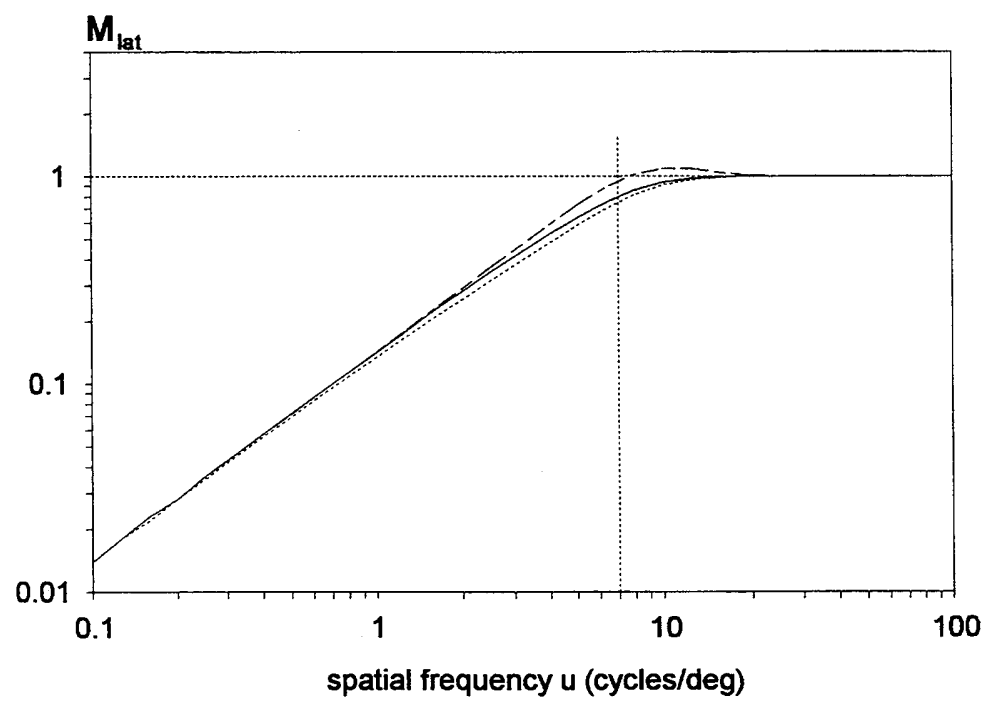

Figure 3.2: Solid curve: MTF of the lateral inhibition process calculated with the approximation formula given by Eq. (3.19) with $u_{0}=7$ cycles/deg. Dotted curve: approximation calculated with the low-pass filter given by Eq. (3.23). Dashed curve: approximation calculated with the annular low-pass filter given by Eq. (3.24). 
As the MTF of the lateral inhibition process is the result of the subtraction of a lowpass filtered signal from a signal that is directly obtained from the photoreceptors, the MTF of the lateral inhibition process may also be described by

$$
M_{\text {lat }}(u)=1-F(u)
$$

where $F(u)$ is the MTF of the spatial lowpass filter. Combination of Eqs. (3.19) and (3.20) gives

$$
F(u)=1-\sqrt{1-\mathrm{e}^{-\left(\mathrm{u} / \mathrm{u}_{0}\right)^{2}}}
$$

The point-spread function that gives such an MTF can be found by an inverse Hankel transform of this expression. The result can numerically be calculated but cannot be represented in mathematical form. This becomes, however, possible, if Eq. (3.21) is replaced by the following expression:

$$
F(u)=0.5 \mathrm{e}^{-2 \mathrm{u} / \mathrm{u}_{0}}+0.5 \mathrm{e}^{-\left(\mathrm{u} / \mathrm{u}_{0}\right)^{2}}
$$

An inverse Hankel transformation of this expression gives

$$
f(r)=\frac{0.25 \pi u_{0}^{2}}{\left(1+\pi^{2} u_{0}^{2} r^{2}\right)^{3 / 2}}+0.5 \pi u_{0}^{2} \mathrm{e}^{-\pi^{2} u_{0}^{2} r^{2}}
$$

This function describes the receptive field of the inhibition process. The MTF of the lateral inhibition process obtained with this function is shown by the dotted curve in Fig. 3.2. This curve nearly coincides with the curve given by Eq. (3.19).

After the classical DOG model, in recent years a model consisting of a ring of Gaussians has been introduced for the description of the lateral inhibition process. See, for instance, Young (1991). This model is called DOOG model (difference of offset Gaussians). These Gaussians form together an annular shaped lowpass filter, instead of the continuous Gaussian lowpass filter used in the DOG model. An annular lowpass filter seems to give a better description of the lateral inhibition process. The lowpass filter given by Eq. (3.23) can be changed in an annular filter by modifying Eq. (3.23) into

$$
f(r)=\frac{0.25 \pi u_{0}^{2}}{\left(1+\pi^{2} u_{0}^{2} r^{2}\right)^{3 / 2}}+1.5 \pi u_{0}^{2} \mathrm{e}^{-\pi^{2} u_{0}^{2} \mathrm{r}^{2}}-1.75 \pi u_{0}^{2} \mathrm{e}^{-1.75 \pi^{2} u_{0}^{2} \mathrm{r}^{2}}
$$

A Hankel transform of this expression gives

$$
F(u)=0.5 \mathrm{e}^{-2 \mathrm{u} / \mathrm{u}_{0}}+1.5 \mathrm{e}^{-\left(\mathrm{w} / \mathrm{u}_{0}\right)^{2}}-1.0 \mathrm{e}^{-\frac{1}{1.75}\left(\mathrm{w} / \mathrm{u}_{0}\right)^{2}}
$$

This function gives a slightly different description of the MTF of the lateral inhibition process than Eq. (3.19). The MTF obtained in this way is shown by the dashed curve in Fig. 3.2.

Fig. 3.3 shows a cross-section of the total point-spread function of the eye obtained by a combination of the optical point-spread function of the eye with the 


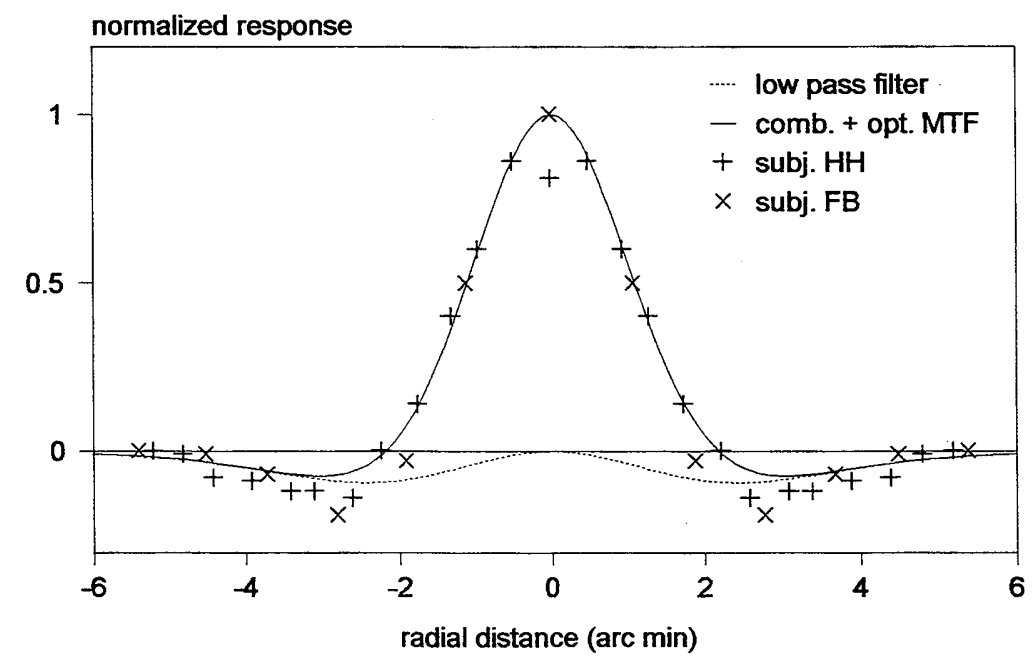

Figure 3.3: Solid curve: cross-section of the total point-spread function of the eye obtained by a combination of the optical point-spread function of the eye with the annular point-spread function of the low-pass inhibition filter given by Eq. (3.23). Dotted curve: cross-section of the annular point-spread function of the lowpass inhibition filter. Data points: measurements of the total point-spread function by Blommaert et al. (1987). For the calculation of the solid curve, the $\sigma$ of the optical point-spread function has been adapted to the measurements.

point-spread function of the annular lowpass filter given by Eq. (3.24). The shape of the annular lowpass filter is shown by the dotted curve in this figure, which is plotted with a negative sign. For $u_{o}$ the mentioned value of $7 \mathrm{cycles} / \mathrm{deg}$ is used. The figure further shows measurement data of the total point-spread function given by Blommaert et al. (1987). These data were obtained with a sophisticated perturbation technique based on peak detection of a combination of sub-threshold stimuli. The measurements were made with an artificial pupil of $2 \mathrm{~mm}$ and a retinal illuminance of $1200 \mathrm{Td}$. The value used for $\sigma$ in the calculated point spread-function has been adapted to the measurements and appears to be somewhat higher than the usual value of 0.5 arc min. Apart from this, the calculations reasonably agree with the measurements. However, the measurements show slightly deeper negative side lobes.

Although the annular filter might give a somewhat better description of the receptive field of the lateral inhibition process, still some uncertainties remain. Therefore, and for the sake of simplicity, still the simple formula given by Eq. (3.19) will be used in the model. 


\subsection{Monocular versus binocular vision}

In comparing visual thresholds, it is important to take into account wether the observation is made with one eye, or with both eyes. At binocular vision the information of both eyes is combined, while the internal noise of both eyes is not correlated. This can be considered as a doubling of the effective integration area. According to Eq. (2.43), the modulation of the internal noise is then reduced with a factor $\sqrt{2}$. So, the contrast sensitivity for binocular viewing increases with a factor $\sqrt{2}$ compared with monocular viewing, if the information of both eyes is indeed completely combined. This appears to be the case. Campbell \& Green (1965) found that the contrast sensitivity for binocular viewing is a factor $\sqrt{2}$ higher than for monocular viewing and these results were later also confirmed by measurements by van Meeteren (1973).

As binocular vision is the most common type of viewing, the factor $\sqrt{2}$ is used as standard in the here given contrast sensitivity model. The contrast sensitivity given by Eq. (3.5) has, therefore to be multiplied with this factor. For monocular vision the contrast sensitivity is a factor $\sqrt{2}$ smaller. If the contrast sensitivity is limited by external noise, the noise presented to both eyes is correlated. Then the contrast sensitivity has also to be taken a factor $\sqrt{2}$ smaller. In this situation it makes no difference if the object is observed with one eye or with two eyes.

\subsection{Complete model}

After correcting Eq. (3.5) with this factor $\sqrt{2}$ and after inserting Eq. (2.48) given in Chapter 2 and the equations given in the preceding sections, the following formula for the spatial contrast sensitivity function at binocular vision is obtained:

$$
S(u)=\frac{1}{m_{\mathrm{t}}(u)}=\frac{M_{\mathrm{opt}}(u) / k}{\sqrt{\frac{2}{T}\left(\frac{1}{X_{\mathrm{o}}^{2}}+\frac{1}{X_{\max }^{2}}+\frac{u^{2}}{N_{\max }^{2}}\right)\left(\frac{1}{\eta p E}+\frac{\Phi_{0}}{1-\mathrm{e}^{-\left(\mathrm{u} / \mathrm{u}_{0}\right)^{2}}}\right)}}
$$

For monocular vision, $S(u)$ is a factor $\sqrt{2}$ smaller. In this equation, $M_{\mathrm{opt}}(u)$ is the optical MTF given by Eq. (3.6), $u$ is the spatial frequency, $k$ is the signal-to-noise ratio, $T$ is the integration time of the eye, $X_{\mathrm{o}}$ is the angular size of the object, $X_{\max }$ is the maximum angular size of the integration area, $N_{\max }$ is maximum number of cycles over which the eye can integrate the information, $\eta$ is the quantum efficiency of the eye, $p$ is the photon conversion factor that depends on the light source and is given in Table 3.2 in Appendix A of this chapter, $E$ is the retinal illuminance in Troland, $\Phi_{0}$ 
is the spectral density of the neural noise, and $u_{0}$ is spatial frequency above which the lateral inhibition ceases. This formula holds for the situation that the object dimensions in $x$ and $y$ directions are equal. For nonequal dimensions, the factor between the brackets that contains the object size has to be replaced by $1 / X Y$ where $X$ and $Y$ are given by Eqs. (2.48) and (2.49), respectively. The typical values of the constants used in the model are:

$$
\begin{array}{lll}
\mathrm{k}=3.0 & T=0.1 \mathrm{sec} & \eta=0.03 \\
\sigma_{0}=0.5 \mathrm{arcmin} & X_{\max }=12^{\circ} & \Phi_{0}=3 \times 10^{-8} \mathrm{sec} \mathrm{\textrm {deg } ^ { 2 }} \\
C_{\mathrm{ab}}=0.08 \mathrm{arcmin} / \mathrm{mm} & N_{\max }=15 \mathrm{cycles} & u_{0}=7 \mathrm{cycles} / \mathrm{deg}
\end{array}
$$

For $T$, it is assumed that the presentation time is long with respect to the integration time of the eye; otherwise Eq. (2.45) has to be used. The given constants are valid for an average observer, foveal vision and photopic viewing conditions. They have been obtained from a best fit with measurement data. For an arbitrary individual subject, only the values of $\sigma_{0}, \eta$, and $k$ have to be adapted.

Fig. 3.4 shows the cumulative effect of various factors on the shape of the contrast sensitivity function. The figure has been calculated with Eq. (3.26) for a field size of $10^{\circ} \times 10^{\circ}$ using the given typical values of the constants. The horizontal line at the top of the figure gives the ultimate limit of the contrast sensitivity for this field size. This limit is determined by neural noise. Lateral inhibition causes a linear attenuation of this limit at low spatial frequencies. The maximum number of cycles

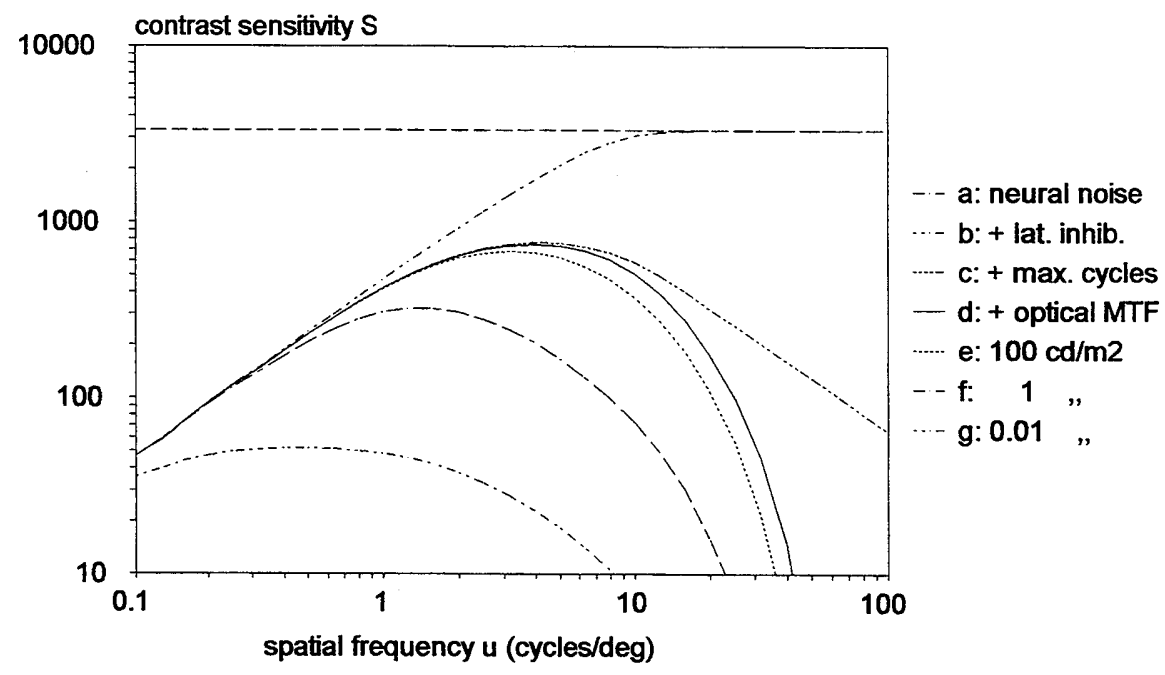

Figure 3.4: Cumulative effect of different factors on contrast sensitivity: (a) neural noise; (b) + lateral inhibition; (c) + limited number of cydes; (d) + optical MTF; (e), (f), and (g) + photon noise at $100 \mathrm{~cd} / \mathrm{m}^{2}, 1 \mathrm{~cd} / \mathrm{m}^{2}$, and $0.01 \mathrm{~cd} / \mathrm{m}^{2}$, respectively. The curves are calculated with Eq. (3.26) for a field size of $10^{\circ} \times 10^{\circ}$. 
causes a decay at high spatial frequencies, which is further enforced by the optical MTF of the eye. Photon noise makes that the contrast sensitivity further decreases and that the contrast sensitivity function changes in shape at lower luminance levels. The figure shows that for low luminance and not too low spatial frequency, the contrast sensitivity increases with the square root of the luminance according to the de Vries-Rose law. The figure also shows that for high luminance or low spatial frequency, the contrast sensitivity is nearly independent of the luminance according to Weber's law. The dependence of the contrast sensitivity on the field size is not shown in the figure. The field size causes a vertical shift of the low frequency part of the curves, whereas the high frequency part remains the same, due to the effect of the limited number of cycles. This will later be shown in sections 3.9.11 and 3.9.14 where the model is compared with contrast sensitivity measurements for different field sizes.

\subsection{Comparison with measurements}

The contrast sensitivity function that can be calculated with the model will now be compared with several published measurements. For the orientation of the sinusoidal test patterns mentioned in these publications, it should be noted that "vertically oriented" means that the bars are vertically oriented and that the sinusoidal luminance variation takes place in horizontal direction. The measurements will be given in chronological order of publication. The constants $\sigma_{0}, \eta$, and $k$ will in each case be adapted to obtain a best fit with the measurements. This fit will be made by trial and error. The constant $k$ appears to influence the fit mainly at low spatial frequencies, $\eta$ appears to influence mainly the fit at medium spatial frequencies, and $\sigma_{0}$ appears to influence mainly the fit at high spatial frequencies. It should be noted that for measurements with a series of data curves, a simultaneous fit will be made for all curves. If the contrast sensitivity is determined with the aid of a 2AFC method where the results do not correspond with $75 \%$ correct response, $k$ will be corrected with Eq. (2.14) given in Chapter 2. A survey of the values used for $\sigma_{0}, \eta$, and $k$ will be given in Table 3.1 at the end of this section.

\subsubsection{Measurements by DePalma and Lowry}

DePalma \& Lowry (1962) measured the contrast sensitivity function at two different luminance levels: $1028 \mathrm{~cd} / \mathrm{m}^{2}$ and $69 \mathrm{~cd} / \mathrm{m}^{2}$ ( $300 \mathrm{ftL}$ and $20 \mathrm{ftL}$ ). The test object was a vertically oriented sinusoidal grating illuminated with a variable luminance and combined with a veiling illumination to obtain a variable contrast. The colour temperature of the illumination was $2850 \mathrm{~K}$ The measurements were made at a viewing distance of 35 inches $(0.89 \mathrm{~m})$ with a field size of $6^{\circ} \times 6^{\circ}$. The observer looked 


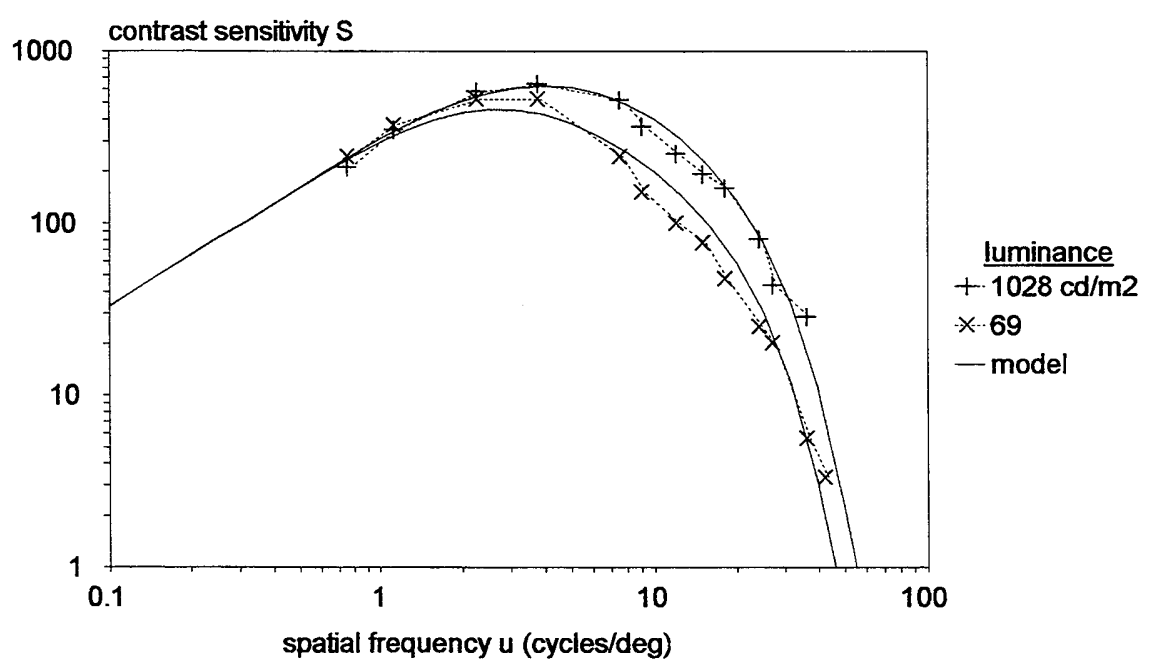

Figure 3.5: Contrast sensitivity function measured by DePalma \& Lowry (1962) at two different luminance levels. Field size $6^{\circ} \times 6^{\circ}$. Binocular viewing with a natural pupil. The solid curves have been calculated with Eq. (3.26).

at the test object with both eyes and without an artificial pupil. The modulation threshold was determined by the method of adjustment.

Measurements and calculations are shown in Fig. 3.5. The values of $\sigma_{0}, \eta$, and $k$ used for the calculation were 0.45 arc min, $0.5 \%$, and 3.0, respectively. The value of $\eta$ is rather low. The general agreement between measurements and calculations is good, although the measured data merge somewhat earlier at low spatial frequencies than the calculated curves.

\subsubsection{Measurements by Patel}

Patel (1966) measured the contrast sensitivity function at four different retinal illuminance levels ranging from $3 \mathrm{Td}$ to $1000 \mathrm{Td}$. The test object was a vertically oriented sinusoidal grating pattern generated on the screen of an oscilloscope tube provided with a green phosphor (P31). The luminance of the pattern was adjusted by using appropriate filters. The measurements were made at a distance of $1 \mathrm{~m}$ with a field size of $2^{\circ} \times 2^{\circ}$. The observer looked at the test object with one eye through an artificial pupil of $2 \mathrm{~mm}$. The modulation threshold was determined by the method of adjustment. Measured data were given for only one subject a male student between 20 and 25 years of age, whose measurement results were considered "typical."

Measurements and calculations are shown in Fig. 3.6. The values of $\sigma_{0}, \eta$, and 


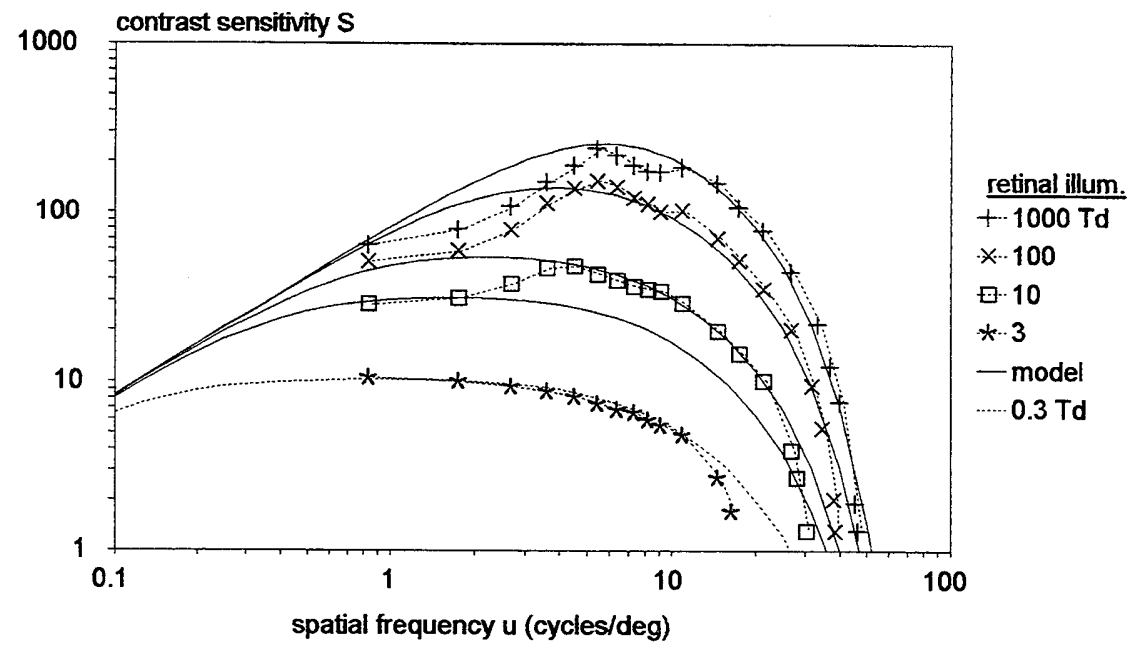

Figure 3.6: Contrast sensitivity function measured by Patel (1966) at four different retinal illuminance levels. Field size $2^{\circ} \times 2^{\circ}$. Monocular viewing with an artificial pupil of $2 \mathrm{~mm}$. The solid curves have been calculated with Eq. (3.26) for the retinal illuminance of the data. The dotted curve gives a fit with the data for $3 \mathrm{~T}$, but has been calculated for $0.3 \mathrm{Td}$.

$k$ used for the calculation were 0.5 arc $\min , 3 \%$, and 3.1 , respectively. The measurements at 10,100, and $1000 \mathrm{Td}$ generally agree with the calculations, apart from a severe dip at about 2 cycles/deg. Such a dip is not found in measurements by other authors, so that it has to be assumed that it was caused by some particular measurement error. This possibility was already mentioned by the authors. Another difference between measurements and calculations is the fact that the measured data for $3 \mathrm{Td}$ do not correspond with calculations for $3 \mathrm{Td}$, but with calculations for $0.3 \mathrm{Td}$ shown by the dotted curve in the figure. This is probably also due to some error.

\subsubsection{Measurements by Robson}

Robson (1966) measured the contrast sensitivity function at a luminance of 20 $\mathrm{cd} / \mathrm{m}^{2}$. The test object was a vertically oriented sinusoidal grating pattern generated on the screen of an oscilloscope tube provided with a green phosphor (P31). Although the grating pattern was simultaneously modulated with a temporal frequency of $1 \mathrm{~Hz}$, this frequency may be assumed to be sufficiently low to consider the measurements as static. See Chapter 5. The angular size of the test object was $2.5^{\circ} \times 2.5^{\circ}$ and the measurements were made at a viewing distance of $1 \mathrm{~m}$. The observer looked at the test object with both eyes and without an artificial pupil. The modulation threshold was probably determined by the method of adjustment. The author himself (a corrected myope) was the observer. 


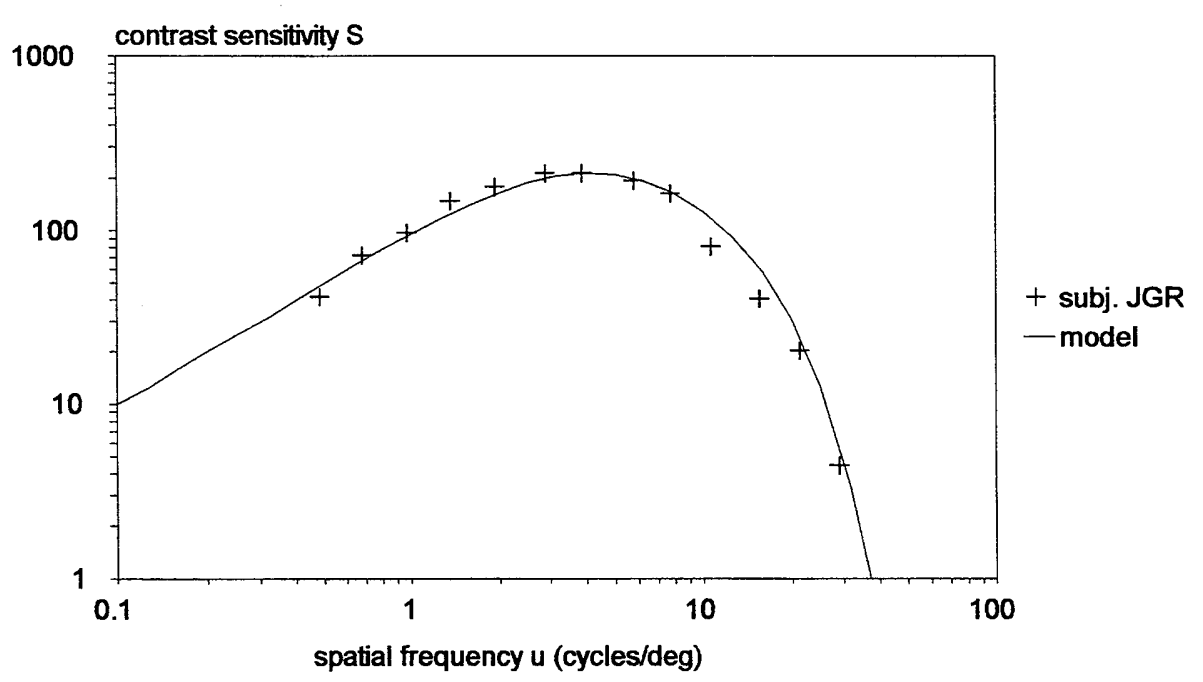

Figure 3.7: Contrast sensitivity function measured by Robson (1966) at a luminance of $20 \mathrm{~cd} / \mathrm{m}^{2}$. Field size $2.5^{\circ} \times 2.5^{\circ}$. Binocular viewing with a natural pupil. The curve through the data points has been calculated with Eq. (3.26).

Measurements and calculations are shown in Fig. 3.7. The values of $\sigma_{0}, \eta$, and $k$ used for the calculation were 0.53 arc $\mathrm{min}, 2.0 \%$, and 4.5 , respectively. The measurements show a very good agreement with the calculations.

\subsubsection{Measurements by van Nes and Bouman}

Van Nes \& Bouman (1967)made similar measurements as Patel, but with monochromatic light and with a larger field size. The measurements were made with monochromatic green light with a wavelength of $525 \mathrm{~nm}$, monochromatic red light with a wavelength of $650 \mathrm{~nm}$, and monochromatic blue light with a wavelength of $450 \mathrm{~nm}$. The measurements with green light extended over a retinal illuminance range of six decades. The test object was a vertically oriented transparent sinusoidal grating illuminated by a variable luminance and combined with a veiling luminance to obtain a variable contrast. The angular size of the test object was $4.5^{\circ}$ in horizontal direction and $8.25^{\circ}$ in vertical direction. The surrounding field was completely dark. The observer looked at the test object with one eye through an optical system with an artificial pupil of $2 \mathrm{~mm}$. The modulation threshold was determined by the method of adjustment where the observer could vary the modulation in steps of one tenth of a decade. In this procedure a lower and a higher limit were determined which appears to differ about $12 \%$ from the arithmetic average of both. See van Nes (1968). The arithmetic average of these limits will be used here as modulation threshold. The first author was the observer. 


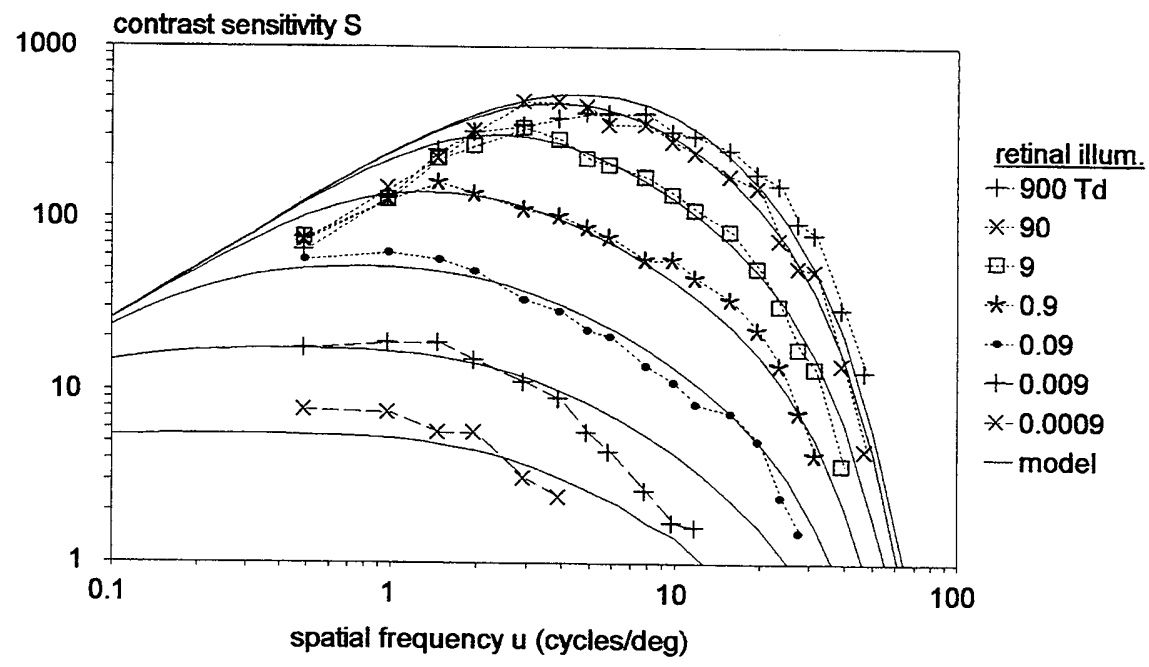

Figure 3.8: Contrast sensitivity function measured by van Nes \& Bouman (1967) over a large range of retinal illuminance levels using monochromatic green light with a wavelength of $525 \mathrm{~nm}$. Field size $4.5^{\circ} \times 8.25^{\circ}$. Monocular viewing with an artificial pupil of $2 \mathrm{~mm}$. The solid curves have been calculated with Eq. (3.26).

Measurements and calculations for green, red, and blue light are shown in Fig.3.8, Fig. 3.9, and Fig. 3.10, respectively. It should be remarked that the light level of the lowest curves in these figures is actually scotopic, whereas the model is only valid for photopic conditions. For all three colours the same values for $\eta$ and $k$ could be used in the calculations $30 \%$ and 2.7 , respectively, whereas for $\sigma_{0}$ different values

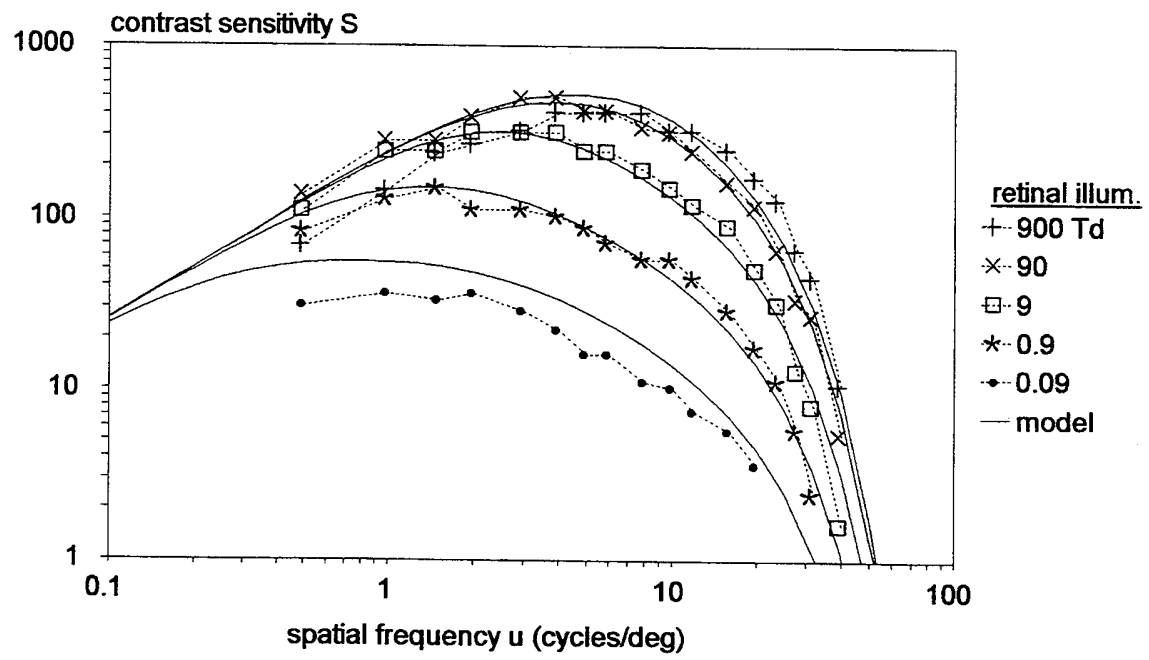

Figure 3.9: Same as Fig. 3.8, but for monochromatic red light with a wavelength of $650 \mathrm{~nm}$. 


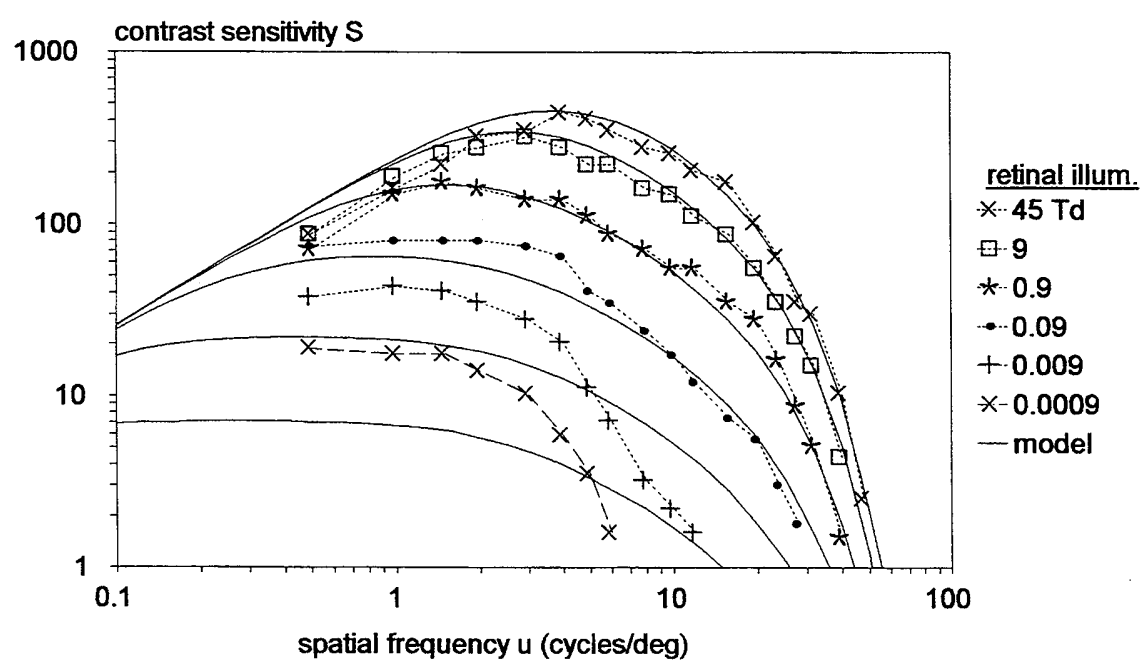

Figure 3.10: Same as Fig. 3.8, but for monochromatic blue light with a wavelength of $450 \mathrm{~nm}$.

had to be used: 0.45 arc min for green light, 0.54 arc min for red light, and 0.50 arc min for blue light. For blue light, a photon conversion factor was used that was derived from the CIE $V_{10}(\lambda)$ curve for fields of $10^{\circ}$ and larger (See Appendix $A$ of this chapter), because of the large field size of the measurements. This factor was used, instead of the factor mentioned in Table 3.2, which is based on the commonly used $V_{2}(\lambda)$ curve that is actually only valid for smaller fields. For the other colours this makes no difference. The value of $30 \%$ for the quantum efficiency $\eta$ of all three colours is unlikely high compared with the results of other investigations. For the more usual value of $3.0 \%$, the retinal illuminance should have been a factor 10 higher.

Measurements and calculations further show a good agreement over a large range of retinal illuminance levels. Not only the vertical position of the calculated curves agrees with the measurements, but also their changing shape at variation of the retinal illuminance. An exception is formed by the lowest curves of which the light level is scotopic, instead of photopic, as assumed in the model. The measurement data for the lowest curves in Figs. 3.9 and 3.10 clearly show the low sensitivity of the rods for red light and the high sensitivity of the rods for blue light, respectively, compared with the sensitivity of the cones used in the calculation. The measurement data for the lowest curves in Figs. 3.8 and 3.10 further show that scotopic resolution is much less than photopic resolution. 


\subsubsection{Measurements by Campbell and Robson}

Campbell \& Robson (1968) measured the contrast sensitivity function at a luminance of $500 \mathrm{~cd} / \mathrm{m}^{2}$. The test object was a vertically oriented sinusoidal grating pattern generated on the screen of a monochrome CRT provided with a white phosphor (P4). The modulating voltage was switched on and off at a rate of $0.5 \mathrm{~Hz}$. This rate may be assumed to be still sufficiently low to consider the presentation as static (See Chapter 5). Measurements for lower spatial frequencies were made at a viewing distance of $0.57 \mathrm{~m}$ with a field size of $10^{\circ} \times 10^{\circ}$, whereas measurements for higher spatial frequencies were made at a distance of $2.85 \mathrm{~m}$ with a field size of $2^{\circ} \times 2^{\circ}$. The observer looked at the test object with one eye through an artificial pupil of $2.5 \mathrm{~mm}$. The modulation threshold was determined by the method of adjustment where the observer varied the modulation until the grating was barely detectable. Either five or ten observations were made to determine each threshold. Both authors served as subjects.

Measurements and calculations for the second author are shown in Fig. 3.11. The values of $\sigma_{0}, \eta$, and $k$ used for the calculation were $0.53 \operatorname{arc} \mathrm{min}, 2.5 \%$, and 3.9 , respectively. For the sake of simplicity all calculations have been made for a field size of $10^{\circ} \times 10^{\circ}$ also for the six highest spatial frequencies measured with a field size of $2^{\circ} \times 2^{\circ}$. For these frequencies this makes hardly any difference. As can be seen from the figure, the measurements show a very good agreement with the calculations. The observer was the same person as the observer at the measurements given in section

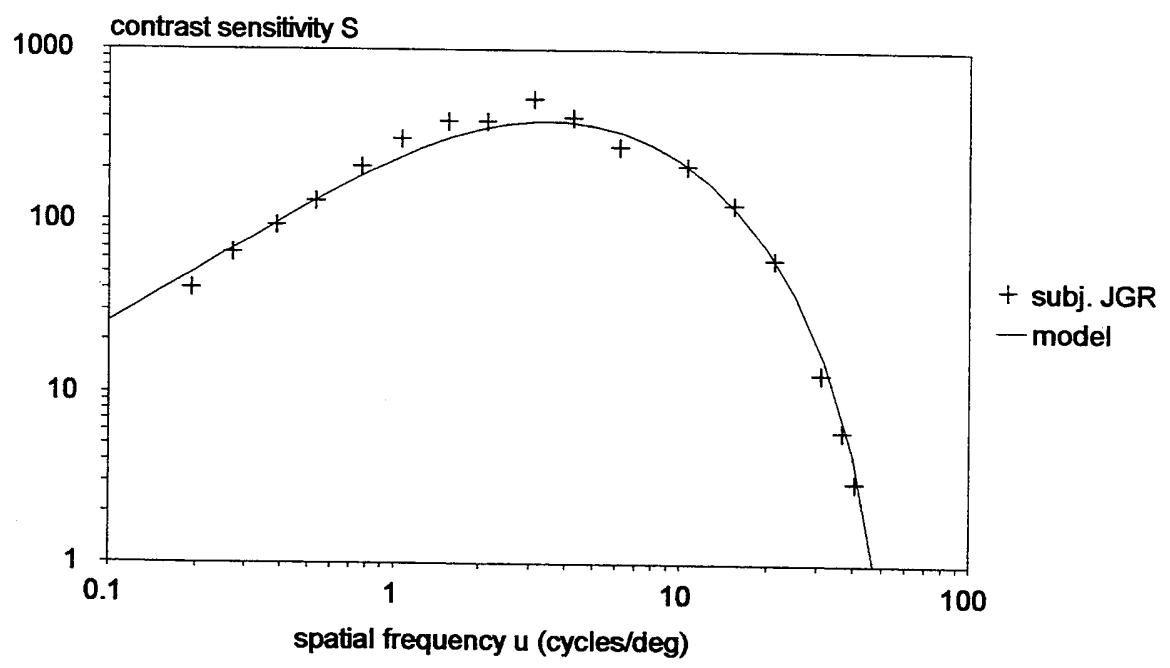

Figure 3.11: Contrast sensitivity function measured by Campbell \& Robson (1968) at a luminance of $500 \mathrm{~cd} / \mathrm{m}^{2}$. Field size $10^{\circ} \times 10^{\circ}$. Monocular viewing with an artificial pupil of $2.5 \mathrm{~mm}$. Subject JGR. The curve through the data points has been calculated with Eq.(3.26). 


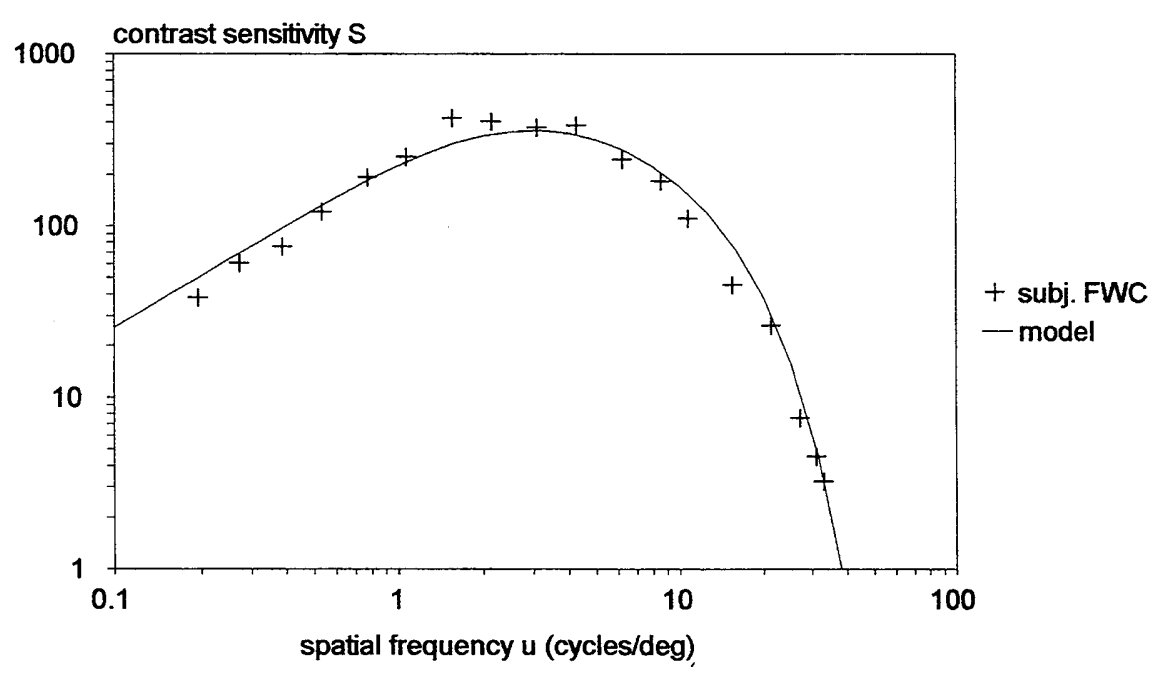

Figure 3.12: Same as Fig. 3.11, but for subject FWC.

3.9.3 where for this subject the same value was found for $\sigma_{0}$ and nearly the same value for $\eta$. These measurements were made under different conditions of luminance and field size and were made with both eyes and a natural pupil.

Measurements and calculations for the first author are shown in Fig. 3.12. The value of $k$ for this observer was the same, but the values of $\sigma_{0}$ and $\eta$ were $0.69 \operatorname{arc} \min$ and $1.0 \%$, respectively. The higher value of $\sigma_{0}$ and the lower value of $\eta$ could be caused by the higher age of this subject.

\subsubsection{Measurements by Watanabe et al.}

Watanabe et al. (1968) measured the contrast sensitivity function at a luminance of $34 \mathrm{~cd} / \mathrm{m}^{2}(10 \mathrm{ftL})$. The test object was a vertically oriented sinusoidal grating pattern generated on the screen of a monochrome TV monitor provided with a white phosphor (P4). The size of the test object was $24 \mathrm{~cm}$ in horizontal direction and 18 $\mathrm{cm}$ in vertical direction. Measurements at lower spatial frequencies were made at a viewing distance of $0.72 \mathrm{~m}$, whereas measurements at higher spatial frequencies were made at a viewing distance of $3.24 \mathrm{~m}$. This corresponded with a field size of $19^{\circ} \times 14^{\circ}$ and $4^{\circ} \times 3^{\circ}$, respectively. The observer looked at the test object with both eyes and without an artificial pupil. The modulation threshold was determined by the method of adjustment where the observer varied the modulation to the point where the grating pattern just disappeared. The reported data are from one of the subjects that took part in the experiments. 


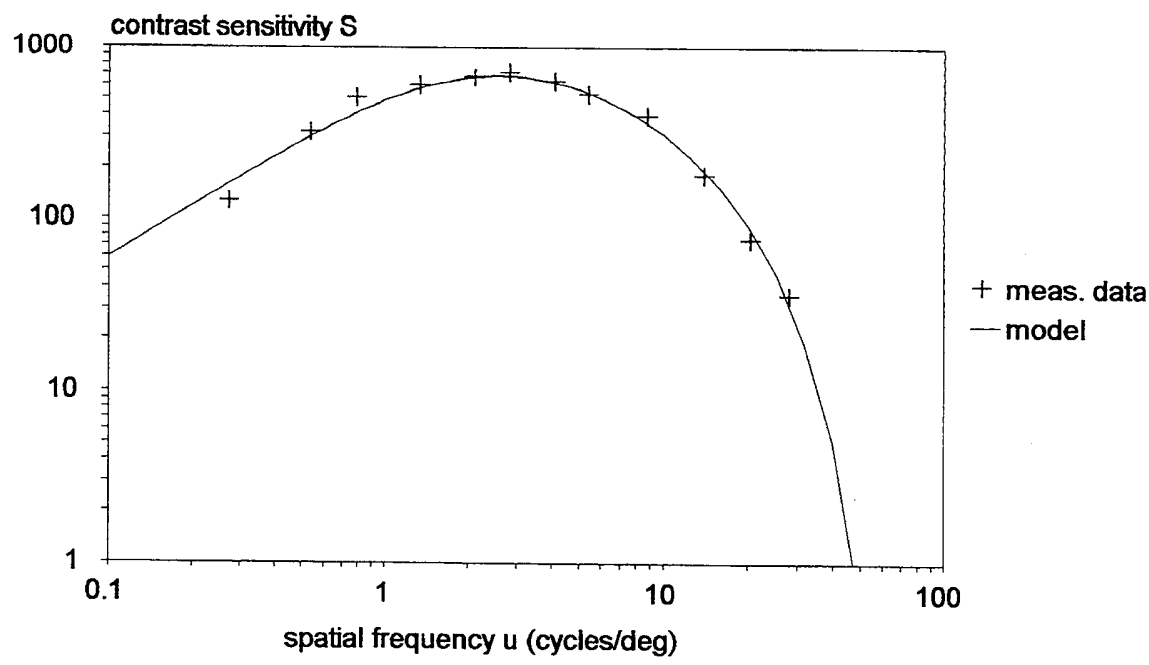

Figure 3.13: Contrast sensitivity function measured by Watanabe et al. (1968) at a luminance of $34 \mathrm{~cd} / \mathrm{m}^{2}$. Field size $19^{\circ} \times 14^{\circ}$. Binocular viewing with a natural pupil. The curve through the data points has been calculated with Eq. (3.26).

Measurements and calculations are shown in Fig. 3.13. The values of $\sigma_{0}, \eta$, and $k$ used for the calculation were 0.48 arc min, $4 \%$, and 3.0, respectively. As with the measurements by Campbell and Robson, the calculations have been made for only the largest field size. This makes also here hardly any difference for the high spatial frequencies. Also these measurements show a very good agreement with the calculations.

\subsubsection{Measurements by Sachs et al.}

Sachs et al. (1971) measured the contrast sensitivity for five different spatial frequencies $(1.4,2.8,5.6,11.2$ and $22.4 \mathrm{cycles} / \mathrm{deg})$ at a luminance of $64 \mathrm{~cd} / \mathrm{m}^{2}(20$ $\mathrm{mL})$. The test object was a vertically oriented sinusoidal grating pattern generated on the screen of a monochrome CRT provided with P31 phosphor. The test object was surrounded by a large cardboard at the same luminance as the stimulus. The measurements were made at a viewing distance of $2.4 \mathrm{~m}$ with a field size of $4.5^{\circ} \times 4.5^{\circ}$. The observer looked at the test object with both eyes and without an artificial pupil. The observer was MS, the first author.

Besides other data, also measurements were given of the psychometric function. From these data not only the contrast sensitivity could be determined, but also the $k$ value that occurred in the measurements. This is different from other published measurements given in this chapter, where only data of the contrast 


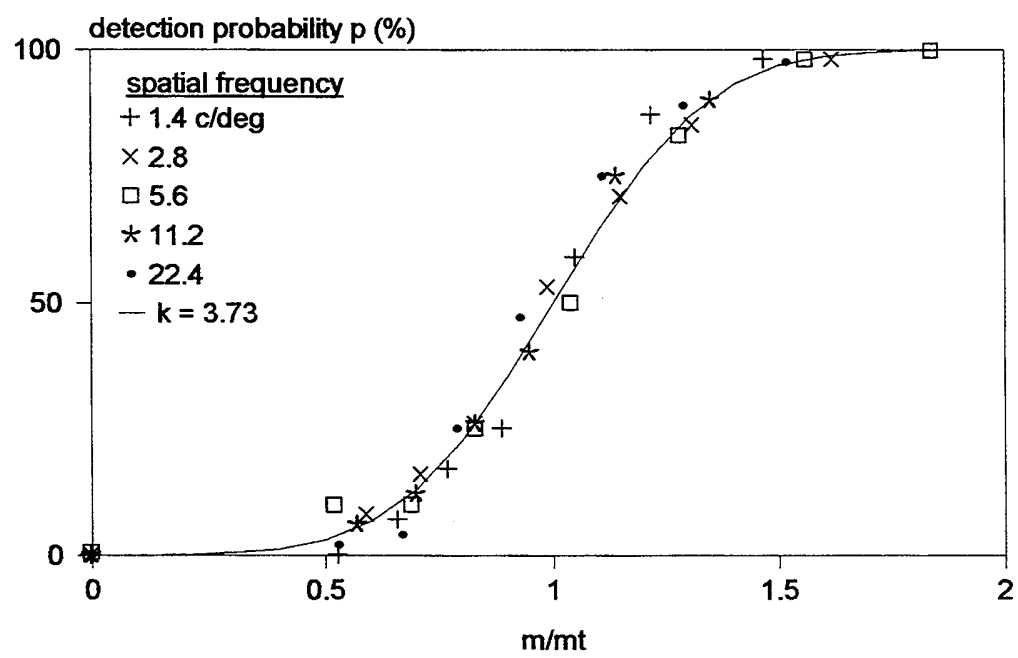

Figure 3.14: Normalized psychometric function for measurements by Sachs et al. (1971) at five different spatial frequencies. The curve through the data points has been calculated with Eqs. (2.2) through (2.4) for the combined results of the normalized data. For this curve $k=3.73$.

sensitivity were available and the $k$ value had to be determined by a best fit with the measurements.

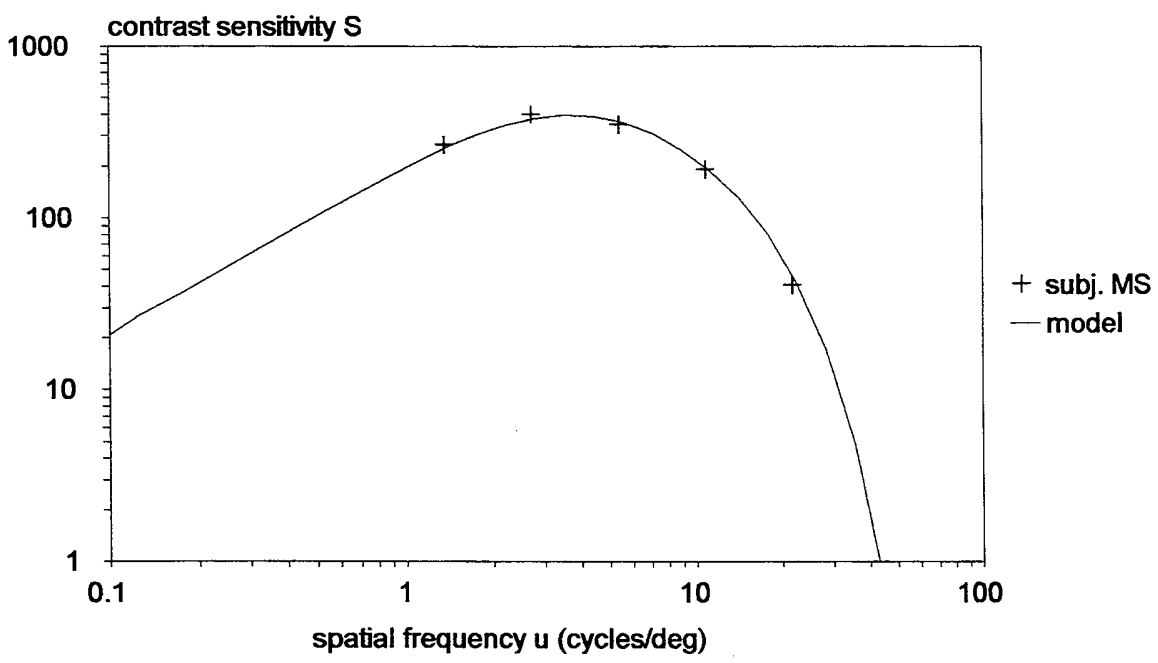

Figure 3.15: Contrast sensitivity function determined from measurements of the psychometric function by Sachs et al. (1971). Luminance $64 \mathrm{~cd} / \mathrm{m}^{2}$. Field size $4.5^{\circ} \times 4.5^{\circ}$. Binocular viewing with a natural pupil. The curve through the data points has been calculated with Eq. (3.26) using for $k$ the value determined from the psychometric function for the combined set of data. 
Fig. 3.14 shows the psychometric functions for the five spatial frequencies, plotted in a normalized way as a function of $\mathrm{m} / \mathrm{m}_{\mathrm{t}}$, as described in Chapter 2 . The curve through the data points has been calculated with Eqs. (2.2) through (2.4) for the combined results of the normalized data. For this curve, the $k$ value appeared to be 3.73 . Fig 3.15 shows the contrast sensitivity obtained from the psychometric functions of these data as a function of the spatial frequency, together with the contrast sensitivity function calculated with the value of $k$ obtained from the combined psychometric function. The values of $\sigma_{0}$ and $\eta$ used for this calculation were 0.59 arc $\min$ and $3 \%$, respectively. The agreement between measurements and calculations shows that the values of $u_{0}$ and $\Phi_{0}$ used in Eq. (3.26) are in good agreement with the measurements, as otherwise a different value of $k$ had to be used to obtain a fit with the data.

\subsubsection{Measurements by van Meeteren and Vos}

Van Meeteren \& Vos (1972) made measurements at various luminance levels similarly as van Nes \& Bouman. However, they used white light, both eyes and a natural pupil, in order to study contrast sensitivity under conditions that were closer to natural vision. The measurements extended over a luminance range of five decades from $10^{-4} \mathrm{~cd} / \mathrm{m}^{2}$ to $10 \mathrm{~cd} / \mathrm{m}^{2}$. The test objects consisted of slides with vertically and horizontally oriented sinusoidal grating patterns projected on a white screen at a distance of $3.5 \mathrm{~m}$ from the observer. The field size was $17^{\circ}$ in horizontal direction and $11^{\circ}$ in vertical direction. A uniform luminance was superimposed on this field by

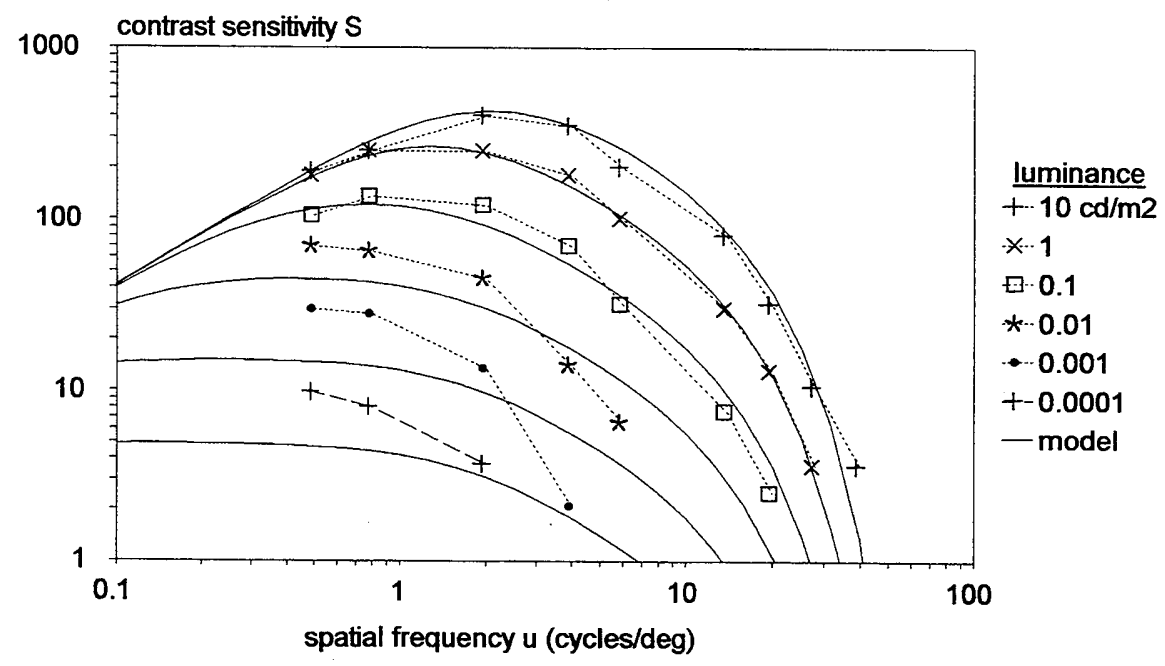

Figure 3.16: Contrast sensitivity function measured by van Meeteren \& Vos (1972) over a luminance range of five decades. Field size $17^{\circ} \times 11^{\circ}$. Binocular viewing with a natural pupil. The solid curves have been calculated with Eq. (3.26). 
a second projector. Modulation and luminance level were partly varied by inserting neutral density filters and partly varied by controlling the lamp currents. The colour temperature of the light source was $2850 \mathrm{~K}$. The observer looked at the test object with both eyes and without an artificial pupil. Horizontal and vertical gratings were presented in random order to the subject, who had to say "horizontal," "vertical," or "no choice." The transition point from "no choice" answers to correct answers was used as threshold. This threshold appeared to correspond with $75 \%$ correct response in a two-alternative forced-choice experiment. The measurements were made by two subjects. The results were averaged over both subjects and both pattern orientations.

Measurements and calculations are shown in Fig. 3.16. The values of $\sigma_{0}, \eta$, and $k$ used for the calculation were 0.5 arc $\mathrm{min}, 3 \%$, and 4.0 , respectively. The agreement between measurements and calculations for the three highest luminance levels is good. Deviations from the curves for the three lowest luminance levels are caused by the fact that the light level of these curves is scotopic, whereas photopic conditions were assumed in the model.

\subsubsection{Measurements by Howell and Hess}

Howell \& Hess (1978) measured the contrast sensitivity function at a luminance of $100 \mathrm{~cd} / \mathrm{m}^{2}$. The test object was a vertically oriented sinusoidal grating pattern generated on the screen of a television tube provided with a white phosphor ( $\mathrm{P} 4)$. The surrounding area was matched to the stimulus with respect to luminance and colour. The test object had a width of 5 cycles and a height of 20 cycles. In this way the authors obtained a constant number of cycles for the integration area at all spatial frequencies. The spatial frequency was varied by varying the size of the object and by varying the viewing distance. By doing so the viewing distance varied between 0.23 $\mathrm{m}$ and $5.7 \mathrm{~m}$. The observer looked at the test object with both eyes and without an artificial pupil. The modulation threshold was determined by the method of adjustment where the observer could vary the modulation in steps of $0.5 \%$. The data are the averages of at least five measurements. Two subjects took part in the experiment. Both subjects were corrected myopes. The data of one subject, JF, are used here.

Measurements and calculations are shown in Fig. 3.17. The values of $\sigma_{0}, \eta$, and $k$ used for the calculation were 0.47 arc $\min , 3 \%$, and 5.0 , respectively. The measurements show a very good agreement with the calculations. The flattened shape of the curve compared with other measurements is caused by the fact that a fixed number of cycles was used in the experiment, instead of a fixed field size. This reduces the angular field size at increasing spatial frequency. The good agreement between measurements and calculations for this situation shows that also this aspect is very well taken into account in the model. 


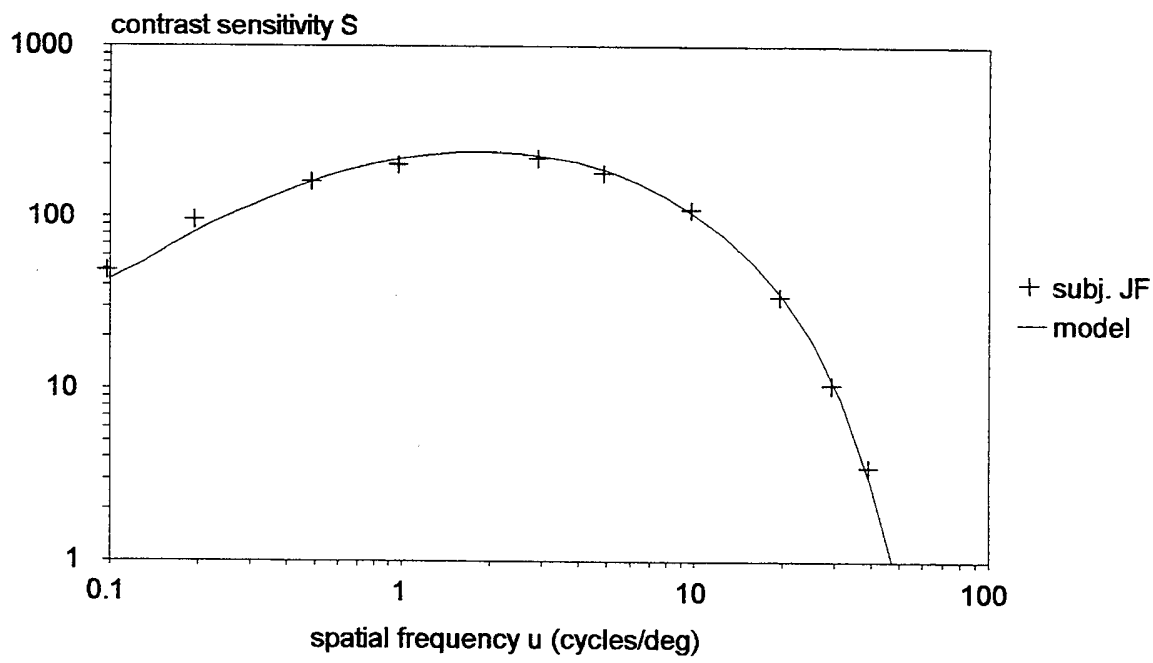

Figure 3.17: Contrast sensitivity function measured by Howell \& Hess (1978) at a luminance of $100 \mathrm{~cd} / \mathrm{m}^{2}$. The test field had a width of 5 cycles and a height of 20 cycles. Binocular viewing with a natural pupil. The curve through the data points has been calculated with Eq. (3.26). The slightly flattened shape is caused by the use of a fixed number of cycles.

\subsubsection{Measurements by Virsu and Rovamo}

Virsu \& Rovamo (1979) measured the contrast sensitivity as a function of the number of cycles at different spatial frequencies. The luminance was $10 \mathrm{~cd} / \mathrm{m}^{2}$. The test object was a vertically oriented sinusoidal grating pattern generated on the screen of a high resolution monitor provided with a white phosphor (P4). The shape of the grating patterns was square except for a few measurements below 1 cycle. In these situations one full cycle was used in horizontal direction, but the height in the other direction was smaller. The luminance of the surrounding area was the same as that of the test object. Viewing distance was varied in addition to a variation of the size of the grating, to achieve a large variation in angular size of the object at all spatial frequencies. The observer looked at the test object with both eyes and without an artificial pupil. The modulation threshold was determined with the aid of a twoalternative forced-choice algorithm where the threshold corresponded with $84 \%$ correct response. Gratings with zero and non-zero modulation were presented in random order. Seven subjects with ages between 25 and 36 participated in the experiments. The reported data are from one subject, the second author.

Measurements and calculations are shown in Fig. 3.18. In this figure contrast sensitivity is plotted as a function of the number of cycles, with spatial frequency as parameter. The values of $\sigma_{0}, \eta$, and $k$ used for the calculation were 0.46 arc min, $2.5 \%$, and 3.2 , respectively, after correcting $k$ for the difference between $84 \%$ and 


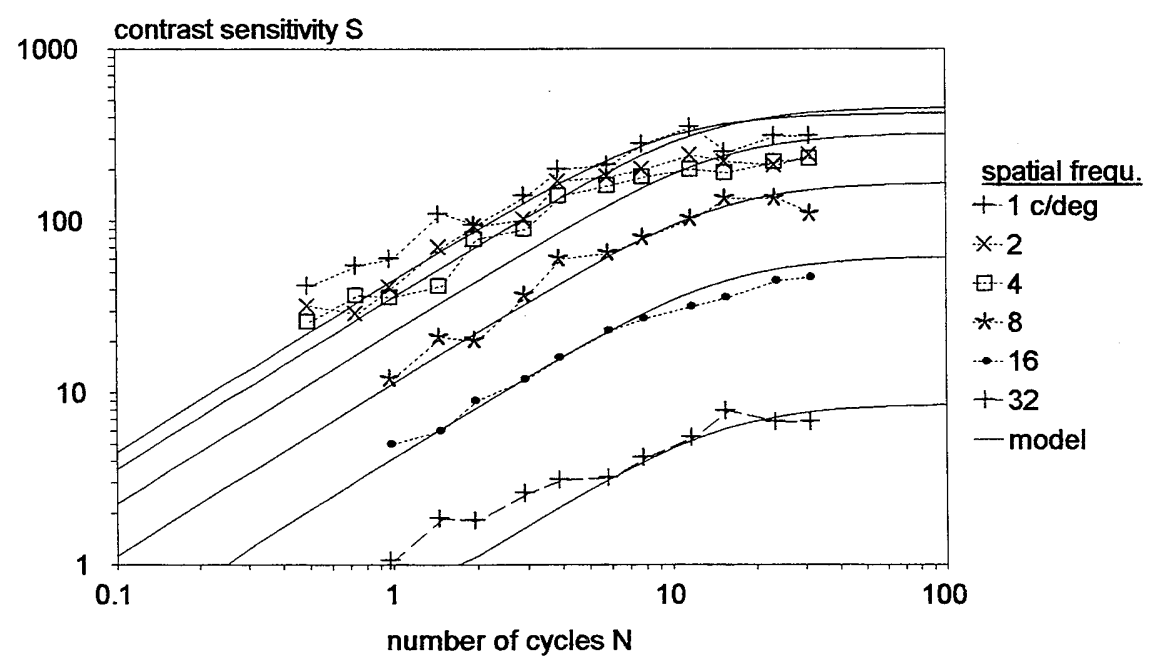

Figure 3.18: Contrast sensitivity as a function of the number of cycles measured by Virsu \& Rovamo (1979) at a luminance of $10 \mathrm{~cd} / \mathrm{m}^{2}$. Binocular viewing with a natural pupil. The solid curves have been calculated with Eq. (3.26).

$75 \%$ correct response with the aid of Eq. (2.14). Apart from the lowest spatial frequencies the calculated curves agree well with the measurements. Measurements and calculations clearly show a saturation of contrast sensitivity at the maximum number of cycles.

\subsubsection{Measurements by Carlson}

Carlson (1982) measured the contrast sensitivity function for a large range of field sizes extending from $0.5^{\circ}$ to $60^{\circ}$. The luminance of the test object was $108 \mathrm{~cd} / \mathrm{m}^{2}$ $(34 \mathrm{~mL})$ with a surrounding luminance of one tenth of this value. The test object was a vertically oriented sinusoidal grating pattern of square size. Patterns with angular field sizes of $0.5^{\circ}, 1.0^{\circ}, 2.3^{\circ}$, and $6.5^{\circ}$ were generated on the screen of a monochrome television monitor, whereas patterns with angular field sizes of $6.5^{\circ}$ and $60^{\circ}$ were projected on the screen of an optical projection system. Both types of measurements gave the same results at an angular size of $6.5^{\circ}$. In both situations the viewing distance was $1.9 \mathrm{~m}$. The observer looked at the test object with both eyes and without an artificial pupil. The modulation threshold was determined by the method of adjustment. The measurements were made with two subjects, one of which was the author. Ten readings were taken at each measurement point for each observer, and the results were averaged. 


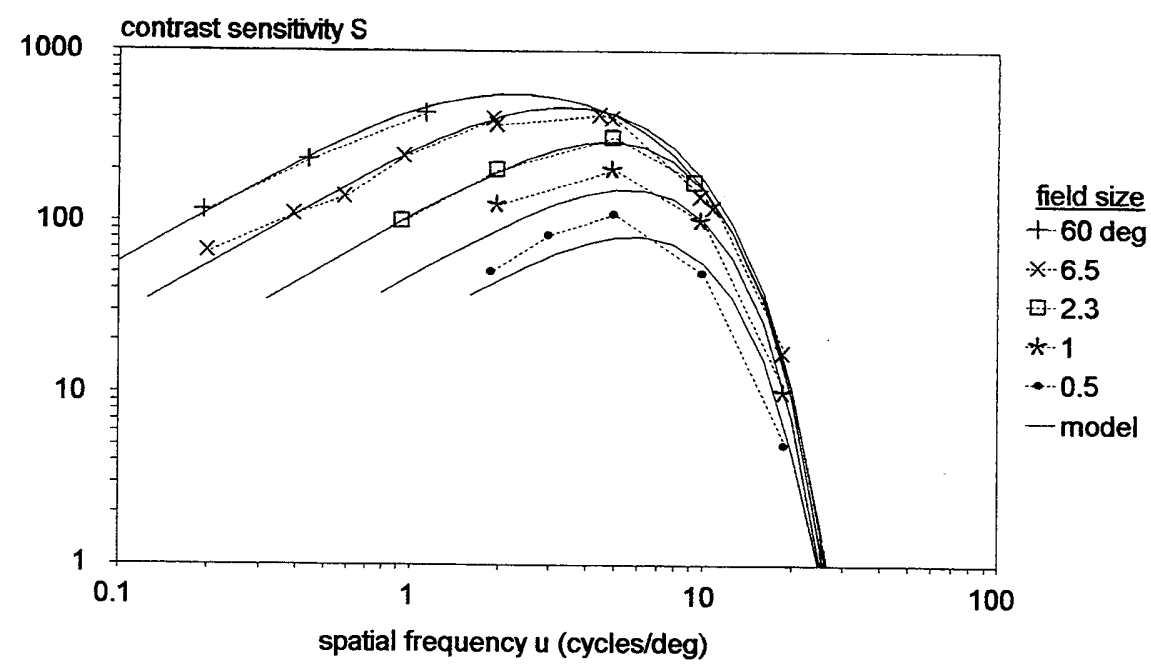

Figure 3.19: Contrast sensitivity function measured by Carlson (1982) at a luminance of $108 \mathrm{~cd} / \mathrm{m}^{2}$ for square test fields with a large range of angular sizes. Binocular viewing with a natural pupil. The solid curves have been calculated with Eq. (3.26).

Measurements and calculations are shown in Fig. 3.19. The values of $\sigma_{0}, \eta$, and $k$ used for the calculation were 1.1 arc $\min , 3.0 \%$, and 3.8 , respectively. The value of $\sigma_{0}$ is very high compared with other measurements. It was probably caused by the fact that one of the two subjects used very strong glasses. The measurements show a good agreement with the calculations. They further show a good illustration for the dependence of contrast sensitivity on field size. At low spatial frequencies, contrast sensitivity decreases with decreasing field size. At high spatial frequencies, the effect of field size gradually disappears. Furthermore, the position of the maximum of the contrast sensitivity function shifts to higher spatial frequencies at smaller field sizes. As the measurements extend to very large angular field sizes, they have been used to determine the value of $X_{\max }$ used in the model.

\subsubsection{Measurements by Rovamo et al. (1992)}

Rovamo et al. (1962) measured the contrast sensitivity function with and without two-dimensional static noise. The test object was a square-shaped vertically oriented sinusoidal grating pattern generated on the screen of a high resolution colour CRT of which only the green phosphor was used. The luminance was $11 \mathrm{~cd} / \mathrm{m}^{2}$. The size of the test object was constant. It contained 16 cycles with a spatial frequency of 1.5 cycle/cm. The angular spatial frequency was varied by changing the viewing distance. This means that simultaneously the angular field size was varied. The test object had an equiluminous surrounding area of $33 \mathrm{~cm} \times 24 \mathrm{~cm}$. Noise was produced by adding 
to each pixel a random luminance value from an even distribution with zero mean. The pixel size was $0.53 \mathrm{~mm} \times 0.53 \mathrm{~mm}$ on the screen. The relative sigma of the noise was 0.289 . The observer looked at the test object with both eyes and without an artificial pupil. The modulation threshold was determined with the aid of a twoalternative forced-choice algorithm where the threshold corresponded with $84 \%$ correct response. The contrast sensitivity was not expressed in the usual way as the inverse of the modulation but as the inverse of RMS contrast. This means that the given contrast sensitivity values had to be divided by a factor $\sqrt{2}$. Two experienced subjects (RF and JR), 24 and 37 years of age, served as observers. Tthe data from subject JR, the first author, will be used here. This is the same subject as took part in the investigation by Virsu and Rovamo that was treated earlier. At the time of this investigation his age was 25.

Measurements and calculations are shown in Fig. 3.20. The values of $\sigma_{0}, \eta$, and $k$ used for the calculation were 0.47 arc $\mathrm{min}, 3.0 \%$, and 3.5 , respectively, after correcting $k$ for the difference between $84 \%$ and $75 \%$ correct response with the aid of Eq. (2.14). For the calculation of the effect of noise, Eqs. (2.42), (2.43) and (2.50) have been used. The agreement between measurements and calculations is very good. The simultaneous fit with the data with and without noise shows that in the model the right value for $\Phi_{0}$ has been chosen. This value cannot accurately be confirmed by measurements without noise.

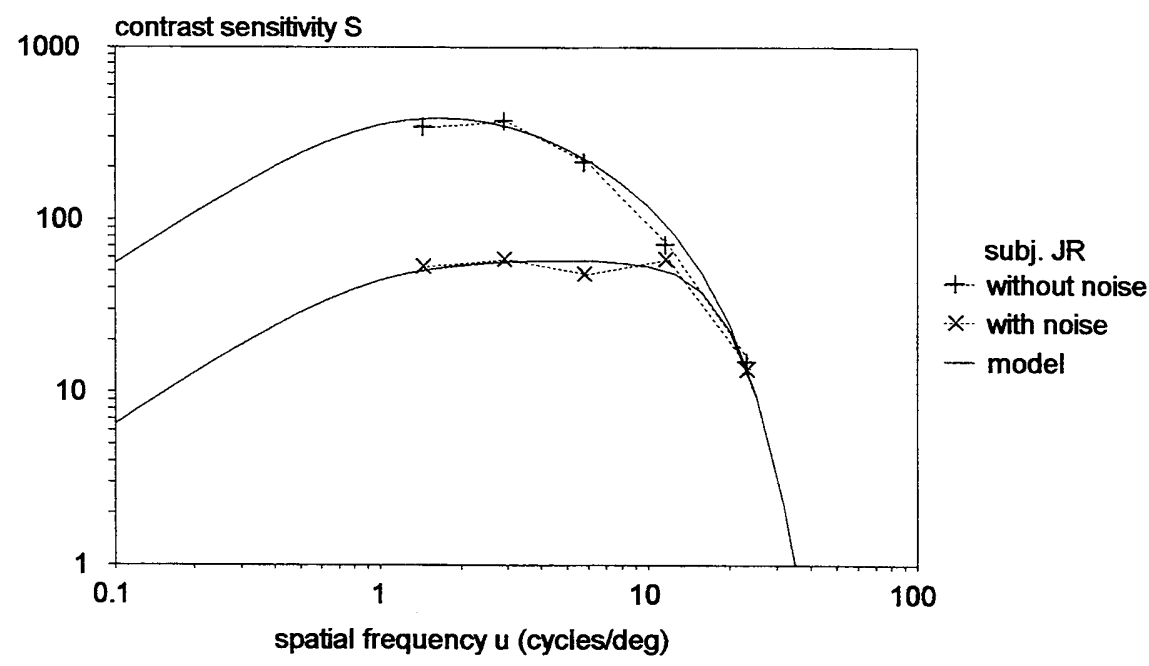

Figure 3.20: Contrast sensitivity function measured by Rovamo et al. (1992) with and without two-dimensional static noise for a square test field containing 16 cycles. Luminance $11 \mathrm{~cd} / \mathrm{m}^{2}$. Binocular viewing with a natural pupil. The solid curves have been calculated with Eq. (3.26). For the calculation of the effect of noise Eqs. (2.42), (2.43) and (2.50) have been used. 


\subsubsection{Measurements by Rovamo et al. (1993a)}

Rovamo et al. (1963a) also measured the contrast sensitivity with and without twodimensional static noise over a wide range of retinal illuminance levels extending over nearly five decades. Only a single spatial frequency was used. The test object was a vertically oriented sinusoidal grating pattern generated on the screen of a high resolution colour CRT used in the white mode. The test pattern had a circular shape with a diameter of $20 \mathrm{~cm}$ and contained 20 cycles and was viewed at a distance of $1.15 \mathrm{~m}$. This corresponded with a circular angular field size of $5^{\circ}$ and a spatial frequency of 4 cycles/deg. Two-dimensional spatial noise was produced by adding to each pixel a random luminance value from an even distribution with zero mean. The pixel size was $0.42 \mathrm{~mm} \times 0.42 \mathrm{~mm}$ on the screen. In one part of the experiment with retinal illumination levels up to $2500 \mathrm{Td}$, the relative sigma of the noise was 0.4 , and in a second part of the experiment with higher retinal illuminance levels, it decreased from this value inversely proportionally with retinal illuminance. The retinal illuminance was varied by using neutral density filters for the lower levels and by adding external light on the screen for the higher levels. Viewing was monocular with the dominant eye of which the pupil was diluted with a drug to a diameter of $8 \mathrm{~mm}$. The modulation threshold was determined with the aid of a two-alternative forcedchoice algorithm where the threshold corresponded with $84 \%$ correct response. As in

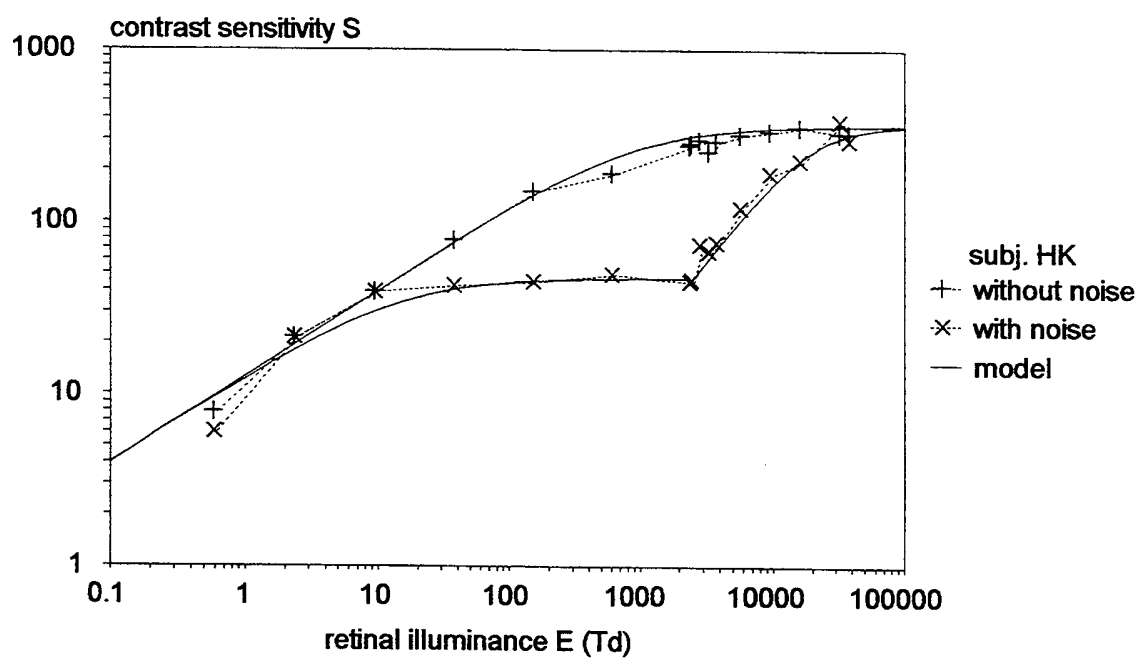

Figure 3.21: Contrast sensitivity with and without two-dimensional static noise measured by Rovamo et al. (1993a) as a function of retinal illuminance. Circular test field with a diameter of $5^{\circ}$ and a spatial frequency of 4 cycles/deg. Monocular viewing with a pupil size of $8 \mathrm{~mm}$. In the measurements with noise, the amount of noise was decreased proportionally with the retinal illuminance above $2500 \mathrm{Td}$. This explains the sudden increase of the contrast sensitivity above this level. The solid curves have been calculated with Eqs. (3.26). For the calculation of the effect of noise Eqs. (2.42), (2.43) and (2.50) have been used. 
the previous experiment, the contrast sensitivity was defined as the inverse of RMS contrast, so that the given contrast sensitivity values had to be divided by a factor $\sqrt{2}$. Two experienced subjects (KT and HK), 25 and 27 years of age, served as observers. The data from subject $\mathrm{HK}$, the second author, will be used here.

Measurements and calculations are shown in Fig. 3.21. In this figure the measurement results of the two parts of the experiment are combined. The sudden increase of the contrast sensitivity above $2500 \mathrm{Td}$ for the measurements with noise is caused by the decrease of noise in the area above this level proportionally with retinal illuminance. The values of $\sigma_{0}, \eta$, and $k$ used for the calculation were 0.50 arc $\min , 1.8 \%$, and 3 , respectively, after correcting $k$ for the difference between $84 \%$ and $75 \%$ correct response with the aid of Eq. (2.14). The agreement between measurements and calculations is very good. As for the previous experiment, a simultaneous fit was made for the curves with and without noise. Also these measurements confirm the value of $\Phi_{0}$ used in the model.

The measurements and calculations for the situation without noise clearly show that the contrast sensitivity increases with the square root of retinal illuminance at low illuminance levels, according to the de Vries-Rose law, and that the contrast sensitivity is constant at high illuminance levels, according to Weber's law. The transition takes place at a level of about $1000 \mathrm{Td}$. This corresponds with a luminance of about $100 \mathrm{~cd} / \mathrm{m}^{2}$ for viewing with a natural pupil. The deviation between measurements and calculations at the lowest illuminance level is caused by the fact that vision at this level is scotopic.

\subsubsection{Measurements by Rovamo et al. (1993b)}

Rovamo et al. (1993b) also measured the dependence of contrast sensitivity on field size, similarly to the measurements made by Carlson. Their measurements extended over a large range of field sizes up to $32^{\circ}$. The luminance was $50 \mathrm{~cd} / \mathrm{m}^{2}$. The test object was a square-shaped vertically oriented sinusoidal grating pattern generated on the screen of a high resolution colour CRT with B22 phosphor used in the white mode (colour coordinates $0.31,0.34$ ). The size of the test object varied from $0.5 \mathrm{~cm}$ $\times 0.5 \mathrm{~cm}$ to $16 \mathrm{~cm} \times 16 \mathrm{~cm}$ and the angular size was further additionally varied by changing the viewing distance. The test object had an equiluminous surrounding area of $27 \mathrm{~cm} \times 20 \mathrm{~cm}$, whereas the further surrounding was completely dark. The observer looked at the test object with both eyes and without an artificial pupil. The pupil size increased with viewing distance from $3.5 \mathrm{~mm}$ to $6 \mathrm{~mm}$. For the calculation an average pupil size of $4.9 \mathrm{~mm}$ was assumed corresponding with an average illuminance level of $940 \mathrm{Td}$. The modulation threshold was determined with the aid of a two-alternative forced-choice algorithm where the threshold corresponded with $84 \%$ correct response. Six experienced subjects from 24 to 33 years of age took part 


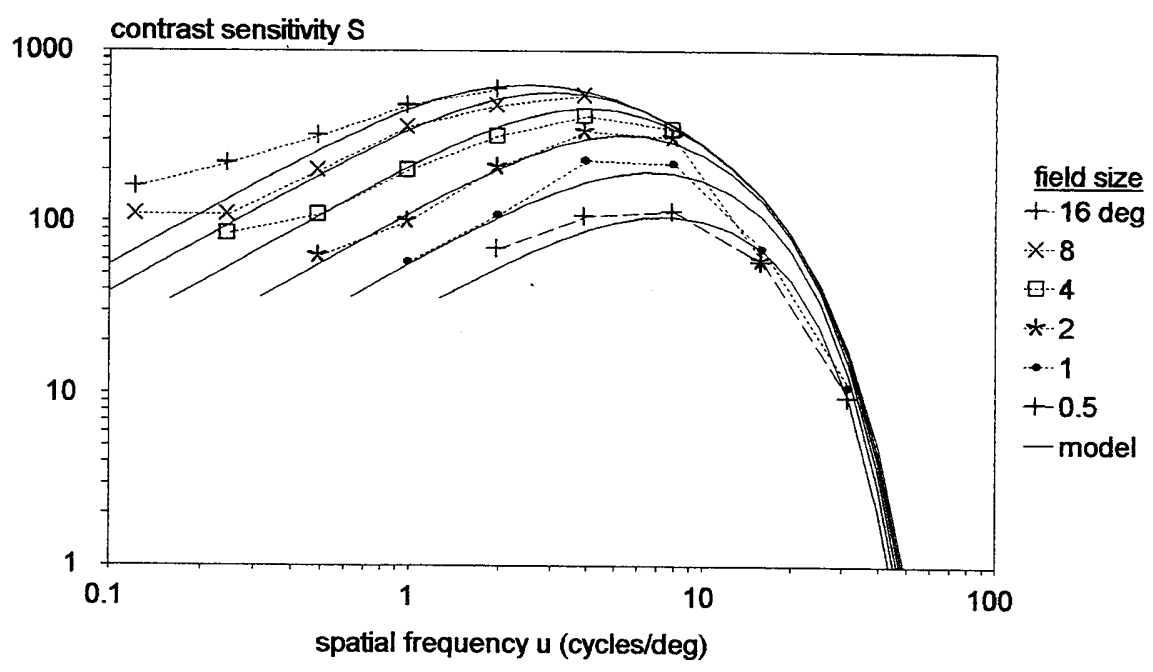

Figure 3.22: Contrast sensitivity function measured by Rovamo et al. (1993b) at a luminance of $50 \mathrm{~cd} / \mathrm{m}^{2}$ for square test fields with a large range of angular sizes. Binocular viewing with a natural pupil. The solid curves have been calculated with Eq. (3.26).

in the investigation.

Measurements and calculations for one subject, the second author, are shown in Fig. 3.22. The values of $\sigma_{0}, \eta$, and $k$ used for the calculation were 0.5 arc $\mathrm{min}, 3 \%$, and 2.7 , respectively, after correcting $k$ for the difference between $84 \%$ and $75 \%$ correct response with the aid of Eq. (2.14). The agreement between measurements and calculations is good, except for the measurements at the lowest spatial frequencies where the data deviate from a linear decay with spatial frequency. Such deviations cannot be found in the measurements by Carlson shown in Fig. 3.19.

\subsubsection{Survey of the measurements}

In the model nine constants play a role, of which six were kept fixed and three were adapted to the measurements, as they could differ for the different subjects. For the fixed constants, the values mentioned in section 3.8 have been used. A survey of the adapted constants is given in Table 3.1. For measurements with a series of data the same constants have been used for all data. The value of $\eta$ is influenced by the measurement accuracy of the luminance or the retinal illuminance. In the past the equipment for measuring the luminance was often not well very well calibrated. The value of $\eta$ for the measurements by van Nes \& Bouman would have been $3 \%$, instead of $30 \%$, if the retinal illuminance was a factor 10 higher. Apart from some exceptions the values of the constants do not much differ from the typical values given in 
section 3.8 .

Table $3.1 \sigma_{0}, \eta$, and $k$ values used for the evaluation of the measurements

\begin{tabular}{|l|c|c|c|}
\hline \multicolumn{1}{|c|}{ author } & $\begin{array}{c}\sigma_{0} \\
(\operatorname{arc} \min )\end{array}$ & $\begin{array}{c}\eta \\
(\%)\end{array}$ & $k$ \\
\hline DePalma \& Lowry (1962) & 0.45 & 0.5 & 3.0 \\
\hline Patel (1966) & 0.50 & 3.0 & 3.1 \\
\hline Robson (1966) & 0.53 & 2.0 & 4.5 \\
\hline van Nes \& Bouman (1967) & 0.45 & 30 & 2.7 \\
& 0.54 & 30 & 2.7 \\
& 0.50 & 30 & 2.7 \\
\hline Campbell \& Robson (1968) & 0.53 & 2.5 & 3.9 \\
& 0.69 & 1.0 & 3.9 \\
\hline Watanabe et al. (1968) & 0.48 & 3.0 & 4.0 \\
\hline Sachs et al. (1971) & 0.59 & 3.0 & 3.7 \\
\hline van Meeteren \& Vos (1972) & 0.50 & 3.0 & 4.0 \\
\hline Howell \&Hess (1978) & 0.49 & 3.0 & 5.0 \\
\hline Virsu \& Rovamo (1979) & 0.46 & 2.5 & 3.2 \\
\hline Carlson (1982) & 1.10 & 3.0 & 3.8 \\
\hline Rovamo et al. (1992) & 0.47 & 3.0 & 3.5 \\
\hline Rovamo et al. (1993a) & 0.50 & 1.8 & 3.0 \\
\hline Rovamo et al. (1993b) & 0.50 & 3.0 & 2.7 \\
\hline
\end{tabular}

\subsection{Summary and conclusions}

In this chapter a model has been given for the spatial contrast sensitivity of the human eye. This model is based on the assumption that contrast sensitivity is limited by internal noise in the visual system. For this model the basic expressions given in the previous chapter have been used for the evaluation of the effect of noise on the modulation threshold. Furthermore, additional assumptions have been made for the 
optical MTF of the eye and for lateral inhibition. This model gives not only a qualitative description but also a quantitative description of the contrast sensitivity function and its dependence on luminance and field size. The results obtained with the model appeared to be in good agreement with a large range of published measurement data, which were used for the comparison. Especially the measurements with and without external noise give a strong support to the model.

In the model, the contrast sensitivity of the eye is explained by effects that mainly take place on retinal level. This does not mean that there would not exist selective spatial frequency channels for different spatial frequency areas and different orientations, as is often assumed. However, from the good agreement between measurements and calculations obtained with the here given model, it appears that many aspects of spatial contrast sensitivity can already be explained without the need to assume such a model.

\section{Appendix A. Photon conversion factor}

The photon conversion factor is defined by the number of photons per unit of time, per unit of area, and per unit of luminous flux per angular area entering the eye. It can be derived from basic photometric and physical quantities. See, for instance, Scheibner \& Baumgardt (1967). For the luminous flux per angular area use will be made of the Troland that is a measure for the retinal illuminance.

For the energy of a photon holds

$$
\varepsilon=h v=h c / \lambda=1.9858 \times 10^{-16} / \lambda \text { Joule }
$$

where the wavelength $\lambda$ is expressed in nm. Taking into account that 1 Watt $=1$ Joule/sec, this means that for monochromatic light

$$
1 \text { Watt }=\frac{1}{1.9858} \times 10^{16} \lambda \text { photons } / \mathrm{sec}
$$

whereas for non-monochromatic light

$$
\text { lWatt }=\frac{1}{1.9858} \times 10^{16} \frac{\int P(\lambda) \lambda d \lambda}{\int P(\lambda) d \lambda} \text { photons } / \mathrm{sec}
$$

where $P(\lambda)$ is the spectral energy distribution function of the light source.

For monochromatic light and photopic vision holds

$$
1 \text { Watt }=683 V(\lambda) \text { lumen }
$$

where $V(\lambda)$ is the standard spectral sensitivity distribution for photopic vision 
adopted by the CIE (Commission Internationale de l'Éclairage) in 1924. This function has as maximum value 1 at $555 \mathrm{~nm}$. For non-monochromatic light

$$
1 \text { Watt }=683 \frac{\int P(\lambda) V(\lambda) d \lambda}{\int P(\lambda) d \lambda} \text { lumen }
$$

Combining these expressions with Eqs. (3.28) and (3.29), respectively, gives for monochromatic light

$$
1 \text { lumen }=7.373 \times 10^{12} \lambda / V(\lambda) \text { photons } / \mathrm{sec}
$$

and for non-monochromatic light

$$
\text { I lumen }=7.373 \times 10^{12} \frac{\int P(\lambda) \lambda d \lambda}{\int P(\lambda) V(\lambda) d \lambda} \text { photons } / \mathrm{sec}
$$

The luminous flux entering the eye from a small axial angular area $d \Omega$ of the object is

$$
\mathrm{d} F=\frac{\pi d^{2}}{4} L \mathrm{~d} \Omega
$$

where $d$ is the diameter of the pupil and $L$ is the luminance of the object. The retinal illuminance caused by this flux is

$$
E_{\text {ret }}=\frac{\tau \mathrm{d} F}{l^{2} \mathrm{~d} \Omega}
$$

where $\tau$ is the transmission coefficient of the eye media and $l$ is the distance between the nodal point of the eye lens and the retina. From Eq. (3.34) follows that

$$
E_{\text {ret }}=\frac{\tau}{l^{2}} \frac{\pi d^{2}}{4} L
$$

The retinal illuminance is usually expressed in the quantity $E$ in Trolands given by Eq. (3.15). With the aid of this equation one obtains

$$
E_{\mathrm{ret}}=\frac{\tau}{l^{2}} E
$$

If $E$ is expressed in Trolands and $E_{\text {ret }}$ is expressed in lumen $/ \mathrm{m}^{2}$, one obtains with $\tau=0.5$ and $l=17 \mathrm{~mm}$

$$
E_{\text {ret }}=2 \times 10^{-3} E
$$

From Eqs. (3.34) and (3.15) follows that the luminous flux per angular area entering the eye is given by

$$
\frac{\mathrm{d} F}{\mathrm{~d} \Omega}=\frac{\pi d^{2}}{4} L=E
$$

If $E$ is expressed in Trolands and $\mathrm{d} F / \mathrm{d} \Omega$ is expressed in lumen/deg ${ }^{2}$, instead of 
lumen/sterad, one obtains

This gives

$$
\left(\frac{\pi}{180}\right)^{2} \frac{\mathrm{d} F}{\mathrm{~d} \Omega}=10^{6} E
$$

$$
1 \text { Troland }=\left(\frac{\pi}{180}\right)^{2} 10^{-6} \text { lumen } / \mathrm{deg}^{2}=3.0462 \times 10^{-10} \text { lumen } / \mathrm{deg}^{2}
$$

For monochromatic light, one obtains with Eq. (3.32)

$$
1 \text { Troland }=2.246 \times 10^{3} \lambda / V(\lambda) \text { photons } / \mathrm{sec} / \mathrm{deg}^{2}
$$

and for non-monochromatic light, one obtains with Eq. (3.33)

$$
1 \text { Troland }=2.246 \times 10^{3} \frac{\int P(\lambda) \lambda d \lambda}{\int P(\lambda) V(\lambda) d \lambda} \text { photons } / \mathrm{sec} / \mathrm{deg}^{2}
$$

Photons at the extreme ends of the visual spectrum have only a small contribution to the viewing process. Multiplying the contribution of each part of the spectrum by $V(\lambda)$ is, therefore, more convenient. In this way, all contributions are weighted by their relative sensitivity with respect to the most sensitive part of the spectrum where $V(\lambda)=1$. For this photopic weighted number of photons holds for monochromatic light and for non-monochromatic light

$$
\text { I Troland }=2.246 \times 10^{3} \lambda \text { photons } / \mathrm{sec} / \mathrm{deg}^{2}
$$

$$
1 \text { Troland }=2.246 \times 10^{3} \frac{\int P(\lambda) V(\lambda) \lambda d \lambda}{\int P(\lambda) V(\lambda) d \lambda} \text { photons } / \mathrm{sec} / \mathrm{deg}^{2}
$$

For photopic viewing, the photopic weighted number of photons has to be used for the photon conversion factor. This gives for the photon conversion factor for monochromatic light

$$
p=2.246 \times 10^{3} \lambda \text { photons } / \mathrm{sec} / \mathrm{deg}^{2} / \mathrm{Td}
$$

and for the photon conversion factor for non-monochromatic light

$$
p=2.246 \times 10^{3} \frac{\int P(\lambda) V(\lambda) \lambda d \lambda}{\int P(\lambda) V(\lambda) d \lambda} \text { photons } / \mathrm{sec} / \mathrm{deg}^{2} / \mathrm{Td}
$$

For scotopic viewing a scotopic weighted photon conversion factor has to be used. Then the $V(\lambda)$ function in the numerator of Eq. (3.47) has to be replaced by the $V^{\prime}(\lambda)$ function for scotopic vision standardized by the CIE in 1951. This function 
has as a maximum value 1 at $507 \mathrm{~nm}$. Simultaneously the right-hand side of Eq. (3.46) for monochromatic light has to be multiplied by $V^{\prime}(\lambda) / V(\lambda)$.

Usually the luminance is measured in photopic units based on the $V(\lambda)$ function. However, if the luminance is measured in scotopic units based on the $V^{\prime}(\lambda)$ function, Eq. (3.30) has to be replaced by

$$
\text { I Watt }=1700 V^{\prime}(\lambda) \text { lumen }
$$

where also the numerical constant is different because of the different shape of the $V(\lambda)$ function. Consequently, the factor $2.246 \times 10^{3}$ in Eqs. (3.46) and (3.47) has to be replaced by $0.9024 \times 10^{3}$ and $V(\lambda)$ in the denominator of Eq. (3.47) has to be replaced by $V(\lambda)$, whereas the right-hand side of Eq. (3.46) has to be multiplied by $V(\lambda) / V(\lambda)$.

Note that in addition to the standard $V(\lambda)$ curve, which was adopted in 1924 and is in principle only valid for fields with a diameter of $2^{\circ}$, the CIE adopted in 1963 a $V_{10}(\lambda)$ curve to be used for larger fields with a diameter of $10^{\circ}$. The reason for the difference is the presence of macular pigment in the fovea, which mainly absorbs blue light. Therefore, the main difference between the curves is the sensitivity for blue. Outside the fovea the sensitivity for blue light is about a factor two higher. Normal light-meters are based on the standard $V(\lambda)$ curve for $2^{\circ}$.

Table 3.2 Photon conversion factor $p$ for different light sources in $10^{6}$ photons $/ \mathrm{sec} / \mathrm{deg}^{2} / \mathrm{Td}$

\begin{tabular}{|l|c|c|c|c|}
\hline \multirow{2}{*}{ light source } & \multicolumn{2}{c|}{ photopic vision } & \multicolumn{2}{c|}{ scotopic vision } \\
\cline { 2 - 5 } & $\begin{array}{c}\text { per } \\
\text { phot. Td }\end{array}$ & $\begin{array}{c}\text { per } \\
\text { scot. Td }\end{array}$ & $\begin{array}{c}\text { per } \\
\text { phot. Td }\end{array}$ & $\begin{array}{c}\text { per } \\
\text { scot. Td }\end{array}$ \\
\hline monochromatic blue 450 nm & 1.011 & 0.0330 & 12.42 & 0.406 \\
\hline monochromatic green 525 nm & 1.179 & 0.4204 & 1.329 & 0.474 \\
\hline monochromatic green 555 nm & 1.247 & 1.229 & 0.508 & 0.501 \\
\hline monochromatic red 650 nm & 1.460 & 87.34 & 0.0099 & 0.586 \\
\hline illumin. A (colour temp. 2854 K) & 1.285 & 0.891 & 0.671 & 0.466 \\
\hline P1 (green CRT phosphor) & 1.201 & 0.467 & 1.212 & 0.472 \\
\hline P31 (blue-green CRT phosphor) & 1.221 & 0.510 & 1.116 & 0.466 \\
\hline P4 (white CRT phosphor) & 1.240 & 0.432 & 1.287 & 0.449 \\
\hline
\end{tabular}


A survey of the photon conversion factor for different light sources is given in Table 3.2. They have been calculated with these equations and have been used for the evaluation of measured contrast sensitivity data with the here given model. The mentioned values for scotopic viewing are given as general information, as the model may only be used for photopic viewing conditions.

\section{References}

Barten, P.G.J. (1992). Physical model for the contrast sensitivity of the human eye. Human Vision, Visual Processing, and Digital Display III, Proc. SPIE, 1666, 57-72.

Blommaert, F.J.J., Heijnen, H.G.M., and Roufs, J.A.J. (1987). Point spread functions and detail detection. Spatial Vision, 2, 99-1 15.

Bouma, H. (1965). Receptive systems. Mediating certain light reactions of the pupil of the human eye. Ph. D. Thesis, Technical University Eindhoven, The Netherlands. Philips Research Reports Supplements, 5.

Bracewell, R. (1965). The Fourier transform and its applications. McGraw-Hill, New York-San Francisco-Toronto-London-Sydney.

Campbell, F.W. \& Green, D.G. (1965) Monocular versus binocular visual acuity. Nature, 208, No. 5006, 191-192.

Campbell, F.W. \& Robson, J.G. (1968). Application of Fourier analysis to the visibility of gratings. Journal of Physiology, 197, 551-566.

Carlson, C.R. (1982). Sine-wave threshold contrast-sensitivity function: dependence on display size. RCA Review, 43, 675-683.

Crawford, B.H. (1936). The dependence of pupil size upon external light stimulus under static and variable conditions. Proceedings Royal Society, B 121, 376-395.

DePalma, J.J. \& Lowry, E.M. (1962). Sine-wave response of the visual system. II. Sine-wave and square-wave contrast sensitivity. Journal of the Optical Society of America, 52, 328-335.

Enroth-Cugell, C. \& Robson, J.G. (1966). The contrast sensitivity of retinal ganglion cells of the cat. Journal of Physiology, 187, 517-522.

Field, D.J. (1987). Relations between the statistics of natural images and the response properties of cortical cells. Journal of the Optical Society of America A, 4, 2379-2394.

le Grand, Y. (1969). Light, colour and vision. 2nd edition, Chapman and Hall, London.

de Groot, S.G. \& Gebhard, J.W. (1952). Pupil size determined by adapting luminance. Journal of the Optical Society of America, 42, 492-495. 
Howell, E.R \& Hess, R.F. (1978). The functional area for summation to threshold for sinusoidal gratings. Vision Research, 18, 369-374.

Jacobs, D.H. (1944). The Stiles-Crawford effect and the design of telescopes. Journal of the Optical Society of America, 34, 694.

Kumnick, L.S. (1954). Pupillary psychosensory restitution and aging. Journal of the Optical Society of America, 44, 735-741.

van Meeteren, A. (1973). Visual aspects of image intensification. Ph. D. Thesis, Utrecht University, Utrecht, The Netherlands.

van Meeteren, A. (1978). On the detective quantum efficiency of the human eye. Vision Research, 18, 257-267.

van Meeteren, A. \& Vos, J.J. (1972). Resolution and contrast sensitivity at low luminance levels. Vision Research, 12, 825-833.

Moon, P. \& Spencer, D.E. (1944). On the Stiles-Crawford effect. Journal of the Optical Society of America, 34, 319-329.

van Nes, F.L. (1968). Experimental studies in spatiotemporal contrast transfer by the human eye. Ph. D. Thesis, Utrecht University, Utrecht, The Netherlands.

van Nes, F.L. \& Bouman, M.A. (1967). Spatial modulation transfer in the human eye. Journal of the Optical Society of America, 57, 401-406.

Patel, A.S. (1966). Spatial resolution by the human visual system. The effect of mean retinal illuminance. Journal of the Optical Society of America, 56, 689-694.

Robson, J.G. (1966). Spatial and temporal contrast-sensitivity functions of the visual system. Journal of the Optical Society of America, 56, 11417-1 142.

Rose, A. (1942). The relative sensitivities of television pickup tubes, photographic film, and the human eye. Proceedings of the IRE, 30, 293-300.

Rose, A (1948). The sensitivity performance of the human eye on an absolute scale. Journal of the Optical Society of America, 38, 196-208.

Rovamo, J., Franssila, R, and Näsänen, R. (1992). Contrast sensitivity as a function of spatial frequency, viewing distance and eccentricity with and without spatial noise. Vision Research, 32, 631-637.

Rovamo, J., Kukkonen, H., Tiippana, K, and Näsänen, R. (1993a). Effects of luminance and exposure time on contrast sensitivity in spatial noise. Vision Research, 33, $1123-1129$.

Rovamo, J., Luntinen, O., and Näsänen, R. (1993b). Modelling the dependence of contrast sensitivity on grating area and spatial frequency. Vision Research, 33, 2773-2788.

Sachs, M.B., Nachmias, J., Robson, J.G. (1971). Spatial-frequency channels in human vision. Journal of the Optical Society of America, 61, 1176-1186.

Schade, O. (1956). Optical and photoelectric analog of the eye. Journal of the Optical 
Society of America, 46, 721-739.

Scheibner, H. \& Baumgardt, E. (1967). Sur l'emploi en optique physiologique des grandeurs scotopiques. Vision Research, 7, 59-63.

Stiles, W.S. \& Crawford, B.H. (1933). The luminance efficiency of rays entering the eye at different points. Proceedings Royal Society, B 112, 428-450.

Virsu, V. \& Rovamo, J. (1979). Visual resolution, contrast sensitivity, and the cortical magnification factor. Experimental Brain Research, 37, 475-494.

Vos, J.J., Walraven, J., and van Meeteren, A. (1976) Light profiles of the foveal image of a point source. Vision Research, 16, 215-219.

de Vries, H. (1943). The quantum character of light and its bearing upon threshold of vision, the differential sensitivity and visual acuity of the eye. Physica, 10, 553-564.

Watanabe, A., Mori, T., Nagata S., and Hiwatashi, K. (1968) Spatial sine-wave responses of the human visual system. Vision Research, 9, 1245-1263.

Young, RA. (1991). Oh say, can you see? The physiology of vision. Human Vision, Visual Processing, and Digital Display II, Proc. SPIE, 1453, 92-123. 


\section{Chapter 4}

\section{Extension of the contrast sensitivity model to extra-foveal vision}

\subsection{Introduction}

The spatial contrast sensitivity model given in the previous chapter is restricted to the normal situation of foveal vision. In this chapter the model will be extended, so that it can also be used for extra-foveal vision. At foveal vision, the eyes of an observer are directed to an object in such a way that the centre of the object is imaged on the centre of the retina where the contrast sensitivity of the eye is maximum. This process of imaging is called foveal fixation. However, in daily practice, as for instance in traffic, it is also important that the eye can observe objects that are outside the area on which the main attention is directed. Outside the fovea, the contrast sensitivity and the resolution of the eye is much less. To measure the local contrast sensitivity outside the fovea, a marker is usually placed in the object plane and the observer is asked to direct his eye on this marker, while the actual object is placed at some distance from the marker. This distance is usually expressed in an angular measure called eccentricity. and the contrast sensitivity is measured as a function of eccentricity. As the instruction to concentrate the eye on the marker is not always easy to follow, extra-foveal contrast sensitivity measurements usually show more spread than foveal contrast sensitivity measurements.

The contrast sensitivity model given in the previous chapter is valid for the situation that the centre of the observed field is imaged on the centre of the retina. This centre is called the fovea and has a diameter of about $1^{\circ}$. Although this situation is called foveal vision, usually a large part of the observed object is imaged on the retinal area outside the fovea. At extra-foveal vision the centre of the object is imaged outside the fovea, but a part of the image can still cover the foveal area.

For the extension of the contrast sensitivity model to extra-foveal vision, it is sufficient to adapt the constants used in the model as a function of eccentricity. It may be assumed that the variation of the constants with eccentricity is caused by the density variation of the cones and ganglion cells over the retina. Therefore, first some 
approximation formulae will be given for the density distribution of these types of cells over the retina, then the effect of these cell types on the contrast sensitivity will be analyzed, and finally the so extended contrast sensitivity model will be compared with various published measurements.

\subsection{Density distribution of cones and ganglion cells}

For photopic vision the density variation of the cones and the ganglion cells over the retina plays the most important role at the variation of contrast sensitivity with eccentricity. Before going into details about the density distribution of these cells first some geometrical relations will be given that will be used here for both cell types.

\subsubsection{Geometrical relations}

From microscopical investigations of the retina, it appears that the cells are largely arranged in hexagonal patterns with randomly different orientations. See, for instance, Polyak (1957, pp. 268-271) and Curio et al. (1987, Fig. 1). In principle a strictly hexagonal array is not possible, as the cell density varies over the retina. It can, therefore, be found only in local areas where the density is approximately constant. However, the hexagonal pattern may still be used as description of the average local situation.

Under this assumption, the distance $s$ between two neighbouring rows of cells is given by

$$
s=\frac{1}{2} \sqrt{3} d
$$

where $d$ is the centre-to-centre distance of the cells. See Fig. 4.I. The available surface area $A$ per cell is

$$
A=s d=\frac{2}{\sqrt{3}} s^{2}
$$

and the density $N$ of the cells per unit area is

$$
N=\frac{1}{A}=\frac{\frac{1}{2} \sqrt{3}}{s^{2}}
$$

The row distance $s$ is usually expressed in arc min of the corresponding visual angle in the object space, and the density $N$ is usually expressed in the number of cells per $\mathrm{deg}^{2}$ of visual angle. Then 


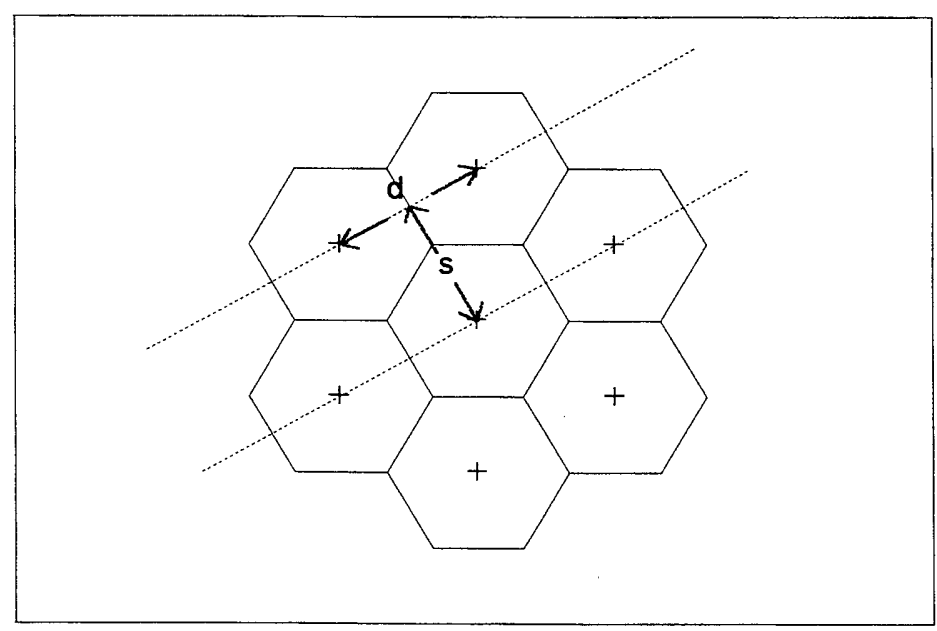

Figure 4.1: Schematic view of the hexagonal structure of the cells on the retina. $d$ is te centre-to-centre distance between the cells and $s$ is the distance between neighbouring rows of cells. Their mutual relation is given by Eq. (4.1).

$$
N=\frac{\frac{1}{2} \sqrt{3} \times 60^{2}}{s^{2}}=\frac{3118}{s^{2}} \mathrm{deg}^{-2}
$$

In biological publications the cell density is usually expressed in cells per square mm on the surface of the retina. The conversion factor from $\mathrm{mm}$ on the retina to the visual angle in the object space amounts to about $0.291 \mathrm{~mm} / \mathrm{deg}$ for the adult human eye. See Williams (1988, footnote, page 441 ). So, for the human eye holds

$$
N=\frac{\frac{1}{2} \sqrt{3} \times 60^{2}}{0.291^{2}} \frac{1}{s^{2}}=\frac{36817}{s^{2}} \mathrm{~mm}^{-2}
$$

The cell density $N$ varies with retinal eccentricity. This eccentricity is usually expressed in degrees.

\subsubsection{Cone density distribution}

Measurements of the density of cones and rods as a function of retinal eccentricity have already been made more than sixty years ago by Østerberg (1935) who investigated tissues of the human retina with the aid of a microscope. The measurement results for the cones are confirmed by more recent measurements in the living eye made by Williams and co-workers (Coletta \& Williams, 1987, Williams, 1988). They 
measured the distance between rows of cones as a function of eccentricity by using the interference between the rows of cones and a grating pattern projected on the retina of the observer, which was visible as a moire pattern. Observers varied the spatial frequency of the grating pattern until the moire pattern was as coarse as possible. This method can be used for eccentricities up to $15^{\circ}$. At larger eccentricities the regularity of the hexagonal array is too much disturbed by local variations to obtain reliable results.

Fig. 4.2 shows the cone density plotted as a function of eccentricity on a double logarithmic scale. The measured data are from Østerberg, Coletta \& Williams, and Williams. The measurements by $\varnothing$ sterberg were given in cells per $\mathrm{mm}^{2}$ at eccentricities in $\mathrm{mm}$. For the conversion from $\mathrm{mm}$ to degrees the factor of 0.291 $\mathrm{mm} / \mathrm{deg}$ mentioned in the section 4.2 .1 was used. The measurements by Coletta \& Williams and Williams were expressed in row spacing. The cone density was calculated from the row spacing with Eq. (4.4). The measurements by Coletta and Williams are the individual results of three observers and the measurements by Williams are the average results of eight observers. From the measurements by Coletta the data for eccentricities above $16^{\circ}$ have been omitted for the reasons mentioned above. The figure shows that the different types of measurements agree very well with each other. The solid curve in the figure has been calculated with the following approximation formula:

$$
N_{\mathrm{c}}=N_{\mathrm{c} 0}\left(\frac{0.85}{1+(e / 0.45)^{2}}+\frac{0.124}{1+(e / 6)^{2}}+0.026\right)
$$

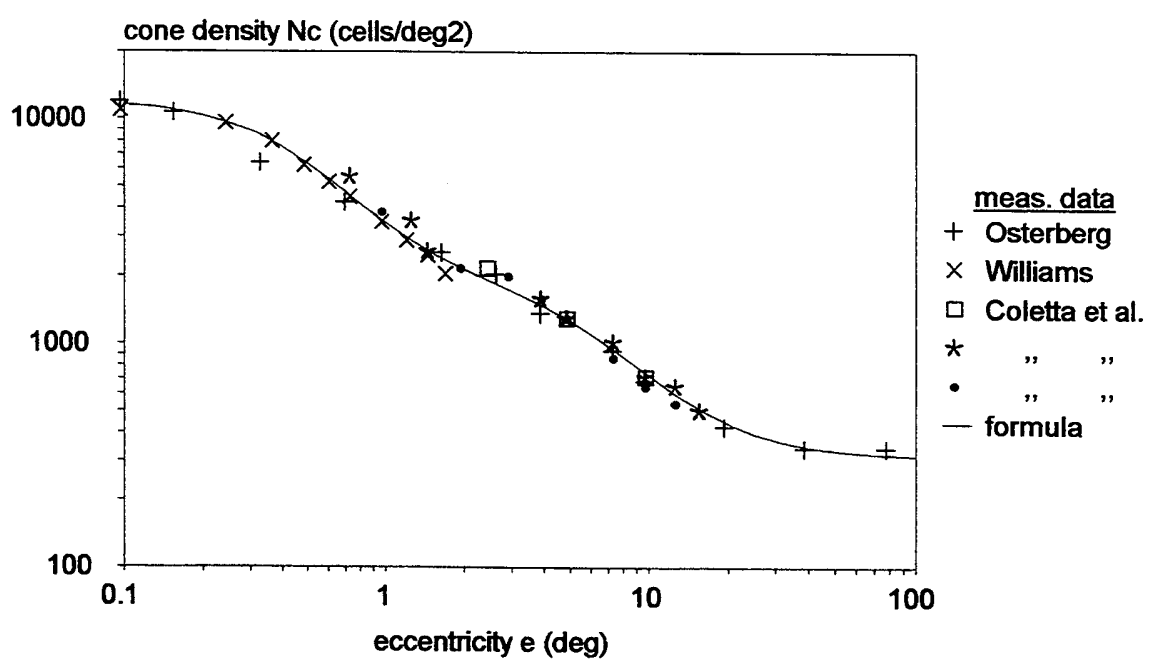

Figure 4.2: Cone density as a function of retinal eccentricity derived from measurements by Østerberg (1935), Williams (1988), and Coletta \& Williams (1987). The curve through the data represents the approximation formula given by Eq. (4.6). 


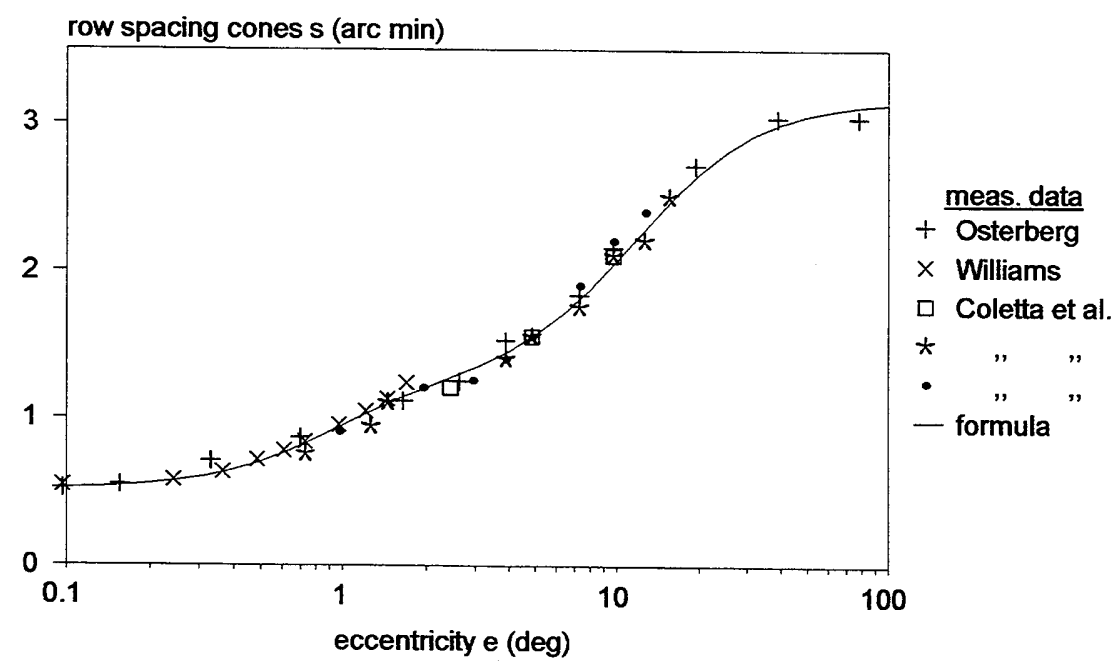

Figure 4.3: Row spacing of cones as a function of retinal eccentricity for the measurement data of Fig. 4.2. The curve through the data points has been calculated with Eqs. (4.6) and (4.4).

where $N_{\mathrm{c}}$ is the cone density, $N_{\mathrm{co}}$ is the cone density in the centre of the retina and $e$ is the eccentricity in degrees. In this formula, the cell density is described as the sum of three overlapping distributions: the density variation in the fovea, the density variation in the area between fovea and periphery, and the density in the periphery, showed in this order by the three terms between brackets. This formula describes the data points very well. The value used for $N_{c o}$ was $12,000 \mathrm{cells} / \mathrm{deg}^{2}$. From the equations given in section 4.2.1 follows that this corresponds for the centre of the retina with a density of 142,000 cells $/ \mathrm{mm}^{2}$, a row spacing $s$ of 0.51 arc min, and a distance $d$ between the centre of the cones of $0.59 \mathrm{arcmin}$. Fig. 4.3 shows the row spacing calculated with Eq. (4.4) for the data given in Fig. 4.2 as a function of the eccentricity. From this figure, it can be seen that the cone spacing varies from about $0.5 \mathrm{arcmin}$ in the fovea to about $3.2 \mathrm{arcmin}$ in the periphery.

\subsubsection{Ganglion cell density distribution}

The ganglion cells in the retina are the cells from which the visual information is transported to the brain. They consist of different types that have different tasks. They can be distinguished (See Henry \& Vidyasagar, 1991) in P-cells, M-cells and Kcells, of which the P-cells are connected with the parvocellular layer of the lateral geniculate nucleus (LGN) in the brain, the M-cells are connected with the magnocellular layer of the LGN and the K-cells are connected with the koniocellular group in the LGN. From the LGN the information is further transported to the visual cortex. According 
to Lee (1996), for the primate retina, $80 \%$ of the ganglion cells are P-cells, $10 \%$ are $\mathrm{M}$-cells and the remaining $10 \%$ are $\mathrm{K}$-cells. The P-cells are responsible for the colour information, the M-cells are responsible for the luminance information and the function of the K-cells is still unknown. The M-cells can be distinguished in on-centre and off-centre cells, which names say that the centre of the receptive field is activated by "light on" and "light off," respectively. Each of them forms $5 \%$ of the total amount of ganglion cells. It is assumed here that this also holds for the human retina. The oncentre M-cells convey the luminance information and are, therefore, responsible for the contrast sensitivity of the eye. They pool the information of several photoreceptors. Therefore, not the density of the photo-receptors itself, but the density of this type of ganglion cells determines the resolution of the eye.

According to Wässle et al. (1990), who investigated the retina of macaque monkeys, there are about three ganglion cells per cone in the centre of the retina. As about $5 \%$ of these cells are on-centre M-cells, there are about 0.15 on-centre M-cells per cone, or about six cones per on-centre M-cell. Because of the resemblance between the eye of the macaque monkey and the human eye, it may be assumed that this number is also valid for the human eye. Using the cone density of 12,000 cells/ $/ \mathrm{deg}^{2}$ in the centre of the retina mentioned in the previous section, the density of the ganglion cells is approximately $3 \times 12,000=36,000$ cells $/ \mathrm{deg}^{2}$ in the centre of the retina and the density of the on-centre $M$ ganglion cells is approximately $0.05 \times$ $36,000=1,800 \mathrm{cells} / \mathrm{deg}^{2}$ in the centre of the retina.

Outside the fovea the ganglion cell density decreases more steeply than the cone density. The decrease is different for the four main directions that are usually distinguished: the nasal and temporal direction along the horizontal meridian of the retina, and the superior and inferior direction along the vertical meridian of the retina. These are the directions of nose and temple, and the upward and downward direction, respectively. Because the image on the retina is an inversed image of the observed object, these directions correspond with opposite directions in the observed field. Curcio \& Allen (1990) made anatomical measurements of the density distribution of ganglion cells in human eyes along these directions. They used eyes of eye bank donors, which they measured within three hours after the death of the donor. The donors were less than 37 years of age. Fig. 4.4 shows the results for the average of six eyes. The given densities are the densities of the total number of ganglion cells. To obtain the densities of the on-centre M-cells, $5 \%$ of this density has to be taken, assuming that this percentage is also valid for the human eye and is constant over the retina. Data for an eccentricity smaller than four degrees have been omitted in the figure, because the ganglion cells at these eccentricities are displaced with respect to the cones with which they are connected. This displacement, which is usually called Henle effect, is caused by an overcrowding of cells in the fovea. There are also no measurements in the nasal area at an eccentricity of $14^{\circ}$ because of the presence of a 


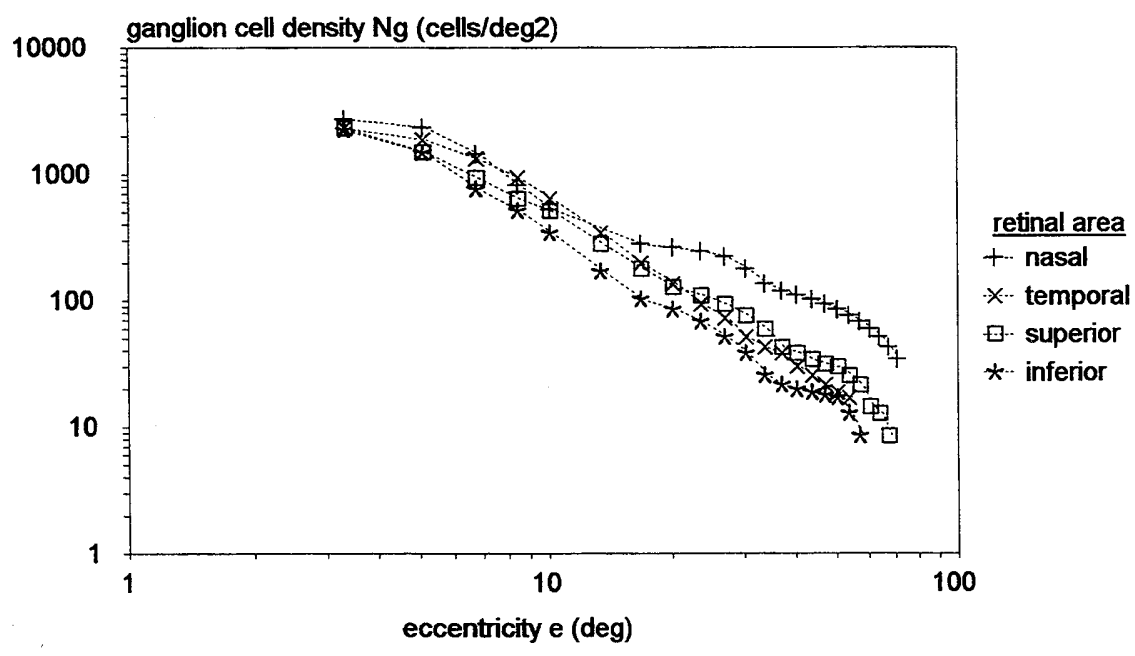

Figure 4.4: Ganglion cell density as a function of eccentricity measured by Curcio \& Allen (1990) for different areas of the human retina.

hole in the retina called the blind spot, which serves for the passage of the nerve fibres. In the remaining part of the nasal area, the ganglion cell density is clearly higher than in the other areas. This can be explained by the need during the evolution of the eye to have a good vision in sideway directions. For probably the same reasons, the density in the inferior area of the retina is less than in the other areas, because the

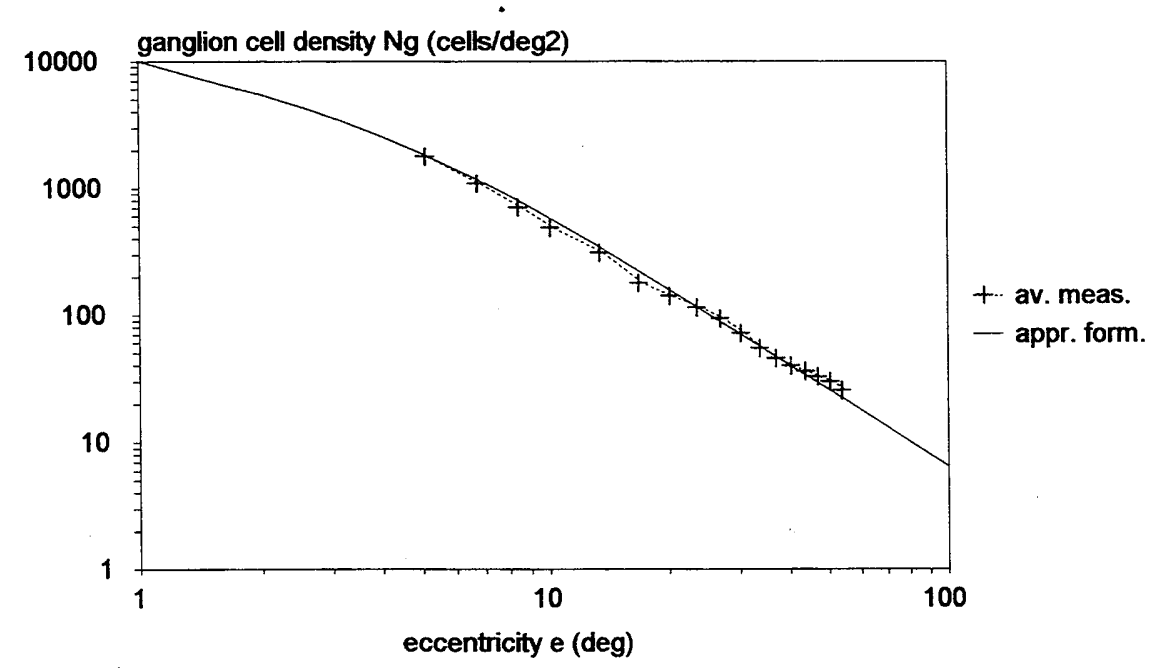

Figure 4.5: Ganglion cell density as a function of eccentricity. Average of the measurements for the different areas given in Fig. 4.4. The solid curve represents the approximation formula given by Eq. (4.7) with $e_{g}=3.3^{\circ}$. 
visual field corresponding with this area is directed to the skies, where less resolution is needed.

The average density variation in the different areas of the retina can be described by an approximation formula that is partly similar as Eq. (4.6) and is given by

$$
N_{\mathrm{g}}=N_{\mathrm{g} 0}\left(\frac{0.85}{1+(e / 0.45)^{2}}+\frac{0.15}{1+\left(e / e_{\mathrm{g}}\right)^{2}}\right)
$$

where $N_{\mathrm{g}}$ is the ganglion cell density, $N_{\mathrm{g} 0}$ is the ganglion cell density in the centre of the retina, $e$ is the eccentricity in degrees, and $e_{\mathrm{g}}$ is a constant that can be different for different subjects. In this expression the first term between brackets is equal to the first term between brackets of Eq. (4.6), because it is assumed here that the ratio between ganglion cells and cones is constant in the foveal area. The function is shown in Fig. 4.5 with the average of the measurements for the different areas given in Fig. 4.4. For $N_{\mathrm{g} 0}$, the value of 36,000 cells/deg mentioned in the previous section is used and for $e_{\mathrm{g}}$ a value of $3.3^{\circ}$. The figure shows that this approximation formula gives a good description of the average of the measured data.

\subsection{Effect of eccentricity on the different constants used in the model}

To extend the use of the contrast sensitivity model to extra-foveal vision, assumptions have to be made about the effect of the eccentricity on the constants for the different parameters used in the model. The variation of these parameters may be assumed to be connected with the variation in density of the different retinal elements with eccentricity.

\subsubsection{Effect of eccentricity on resolution}

As mentioned in section 3.3 of the preceding chapter, the optical MTF used in the model does not include only the optical effect of the eye lens and other optical effects, but also the effect of the discrete structure of the retina. The value of $\sigma$ in Eq. (3.6), which describes the optical MTF, is the standard deviation of the linespread function resulting from the convolution of the different elements by which the optical MTF of the eye is determined. To take the effect of the structure of the retina explicitly into account $\sigma_{0}$ in Eq. (3.7) may be written as

$$
\sigma_{0}=\sqrt{\sigma_{00}^{2}+\sigma_{\text {ret }}^{2}}
$$

where $\sigma_{\text {ret }}$ is the standard deviation of the line-spread function caused by the discrete 
structure of the retina and $\sigma_{00}$ is the standard deviation of the remaining parts of $\sigma_{0}$. In this equation, it is assumed that the standard deviation of the line-spread function resulting from the convolution is equal to the square root of the sum of squares of the standard deviations of the different elements. By inserting Eq. (4.8) in Eq. (3.7), this equation is modified in

$$
\sigma=\sqrt{\sigma_{00^{2}}+\sigma_{r e t}^{2}+\left(C_{\mathrm{ab}} d\right)^{2}}
$$

$\sigma_{\text {ret }}$ can be derived from the size of an elementary area that delivers information to the brain. As already mentioned in the previous section, the on-centre M-cells pool the information of several cones for a further transport to the brain. This means that $\sigma_{\text {ret }}$ is determined by the area covered by these cells. For the ganglion cells, it may be assumed that they are arranged in a hexagonal array similarly as that of the cones. The centre-to-centre distance $s_{\mathrm{g}}$ between rows of ganglion cells can then be derived from the density of the ganglion cells. Using Eq. (4.3), one obtains

$$
s_{\mathrm{g}}=\sqrt{\frac{\frac{1}{2} \sqrt{3}}{N_{\mathrm{g}}}}
$$

Rows of on-centre $M$ ganglion cells form the line-spread function that determines the effect of the retinal structure on the optical MTF of the eye. The $50 \%$ width of the line-spread function is equal to the centre-to-centre distance of neighbouring rows of these ganglion cells. This is shown in Fig. 4.6 where, it is assumed that the collected information is uniformly distributed over the area of the rows. The line-spread function formed by the rows of ganglion cells is shown by the shaded area at the bottom of this figure. The standard deviation of this function can be calculated from an integration over the width of the intensity distribution. From the geometrical configuration given in Fig. 4.6 can be derived in this way that

$$
\sigma_{\text {ret }}=\frac{s_{\mathrm{g}}}{3 \sqrt{6 / 5}}
$$

where $s_{\mathrm{g}}$ is the centre-to centre distance between rows of on-centre $\mathrm{M}$ ganglion cells, and it is assumed that a further overlap of the ganglion cells may be neglected. By replacing $s_{\mathrm{g}}$ in this expression by $N_{\mathrm{g}}$ with the aid of Eq. (4.10) one obtains

$$
\sigma_{\mathrm{ret}}=\frac{1}{\sqrt{7.2 \sqrt{3} N_{\mathrm{g}}}}
$$

where $N_{\mathrm{g}}$ is now the density of the on-centre $\mathrm{M}$ ganglion cells. Applying this equation to the centre of the retina, where the density of these ganglion cells is approximately $1,800 \mathrm{cells} / \mathrm{deg}^{2}$, gives $\sigma_{\mathrm{ret}}=0.40 \mathrm{arc} \min$. In section 3.3 of Chapter 3 , it was mentioned that $\sigma_{0}$ is approximately $0.5 \mathrm{arc}$ min for observers with good vision. From the value of $\sigma_{\text {ret }}$ follows that for these observers $\sigma_{00}=\sqrt{ }\left(0.5^{2}-0.4^{2}\right)=0.30 \mathrm{arcmin}$. This means that for foveal vision and a small size of the eye pupil, the contribution 


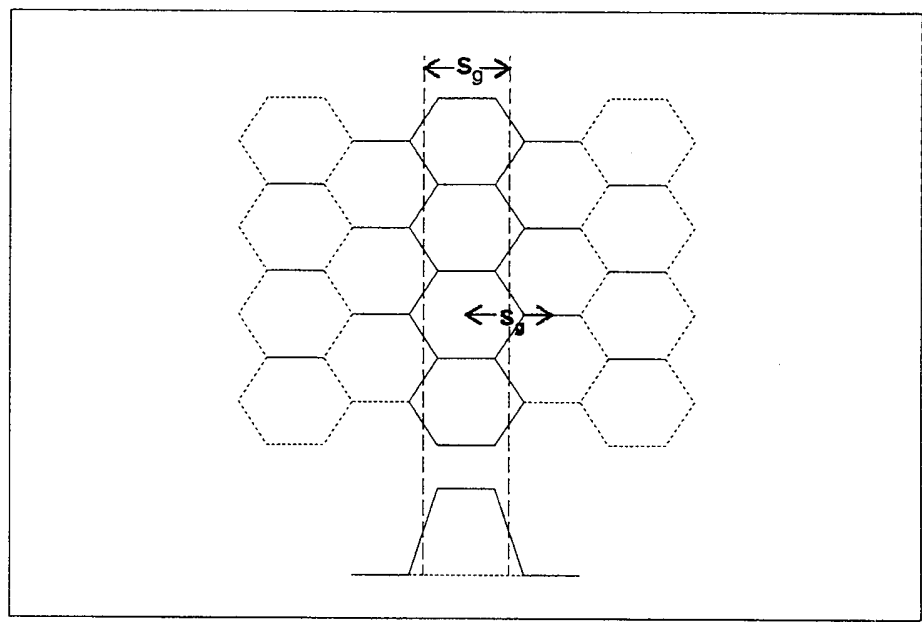

Figure 4.6: Schematic view of a row of on-centre $M$ ganglion cells which determines the effect of the retinal structure on the optical MTF of the eye. The line-spread function formed by this row is indicated by the shaded area at the bottom of the figure. The $50 \%$ width of this function is equal to the centre-to-centre distance $s_{\mathrm{g}}$ between neighbouring rows of these ganglion cells.

of the retinal structure of the eye on the resolution is about equal to that of other factors. The development of the eye during evolution seems, therefore, to be well balanced.

At increasing distance from the centre of the retina, $\sigma_{\text {ret }}$ increases because of the decrease of the ganglion cell density. This means that at some distance from the centre, $\sigma_{\text {ret }}$ becomes dominant and the resolution of the eye becomes completely determined by the density of the ganglion cells, as the other terms in Eq. (4.9) remain nearly constant or increase less with eccentricity. With the aid of Eq. (4.12) the value of $\sigma$ of the optical MTF of the eye can then be calculated from the ganglion cell density at different eccentricities. Inversely, the ganglion cell density at different eccentricities can be calculated from the value of $\sigma$ derived from measurements of the contrast sensitivity at these eccentricities. This method will be used in section 4.4 for the analysis of extra-foveal contrast sensitivity measurements.

\subsubsection{Effect of eccentricity on neural noise}

As already mentioned in section 3.5 of the preceding chapter, neural noise is assumed to be caused by statistical fluctuations of the signals in the nerve fibers by which the luminance information is transported to the brain. By inversion of Eq. (3.18) one obtains 


$$
\Phi_{0}=\sigma^{2} \Delta x \Delta y \Delta t=\frac{\sigma^{2} \Delta t}{N_{\mathrm{g}}}
$$

where $\Delta x \Delta y$ has been replaced by $1 / N_{\mathrm{g}}$ and $N_{\mathrm{g}}$ is the density of the on-centre $\mathrm{M}$ ganglion cells. In this expression $\sigma$ is the relative standard deviation of the signal transported by an individual nerve fibre. If it is assumed, that $\sigma$ and $\Delta t$ do not vary with eccentricity, this expression says that the spectral density of neural noise varies inversely proportionally to the density of the ganglion cells. This means that the spectral density of neural noise can be expressed as a function of eccentricity by the following expression:

$$
\Phi_{0}(e)=\Phi_{0} \frac{N_{\mathrm{g} 0}}{N_{\mathrm{g}}}
$$

where $\Phi_{0}$ at the right-hand side of this expression is the value of $\Phi_{0}$ at foveal vision, given in the previous chapter, and $N_{\mathrm{g}}$ can be calculated with Eq. (4.7), or can be derived from a best fit with measurements of extra-foveal contrast sensitivity functions.

\subsubsection{Effect of eccentricity on lateral inhibition}

From the equations given in section 3.6 of the previous chapter can be calculated that the lowpass filter used in the lateral inhibition process extends over a receptive field with a radius of about 3 arc min. See Fig. 3.3. This holds for foveal vision. At increasing eccentricity not only the mutual distance between the cones increases, but also the size of the receptive field. The spatial frequency $u_{0}$, where the lateral inhibition ceases, varies inversely proportionally with the size of the receptive field and will therefore shift to lower spatial frequencies. One would expect that the diameter of the receptive field would increase proportionally with the distance between the ganglion cells, so that the number of ganglion cells involved in the lateral inhibition process remains about constant. This means that $u_{0}$ would vary proportionally with $\sqrt{ } N_{\mathrm{g}}$. However, as the ganglion cell density decreases rather steeply with increasing eccentricity, this would lead at high eccentricities to a very large field for the lateral inhibition and to a very small value of $u_{0}$. This is not in agreement with measurements. Therefore, it has to be assumed that the receptive field of the inhibition process contains a decreasing number of ganglion cells with increasing eccentricity so that the decrease of $u_{0}$ with eccentricity is limited. From an analysis of the extra-foveal contrast sensitivity measurements given in section 4.4 of this chapter, it appears that the variation of $u_{0}$ with eccentricity may approximately be described by the following empirical formula:

$$
u_{0}(e)=u_{0}\left(\frac{N_{g}}{N_{g o}}\right)^{0.5}\left(\frac{0.85}{1+(e / 4)^{2}}+\frac{0.13}{1+(e / 20)^{2}}+0.02\right)^{-0.5}
$$


where $u_{0}$ at the right-hand side of this expression is the value of the constant at foveal vision, given in the previous chapter, and $e$ is the eccentricity in degrees. The decrease of the number of ganglion cells used in the inhibition process is described by the last factor of this equation.

\subsubsection{Effect of eccentricity on quantum efficiency}

The quantum efficiency of the eye is sometimes defined by the chance that a photon falling on a photon-receptor causes an excitation of this receptor. However, according to the definition used here, the quantum efficiency is defined by the average number of photons causing an excitation of the photo-receptors, divided by the number of photons entering the eye. See section 3.4. This means that the quantum efficiency also depends on the area of the retina covered by the photo-receptors. In the centre of the fovea hardly any rods are present, so that nearly the complete area is covered with cones. This makes that the quantum efficiency in the centre of the fovea is very low for the rods, but is maximum for the cones. Outside the centre of the retina the area covered by the rods increases, and consequently the area covered by the cones decreases, so that the quantum efficiency of the rods increases and that of the cones decreases. In the central area of the retina the quantum efficiency of the cones is flattened by the presence of macular pigment in the foveal area, which acts as a filter for blue light. From an evaluation of the contrast sensitivity measurements given in section 4.4 of this chapter, it appeared that the dependence of the quantum efficiency of the cones with eccentricity can approximately be described by the following empirical formula

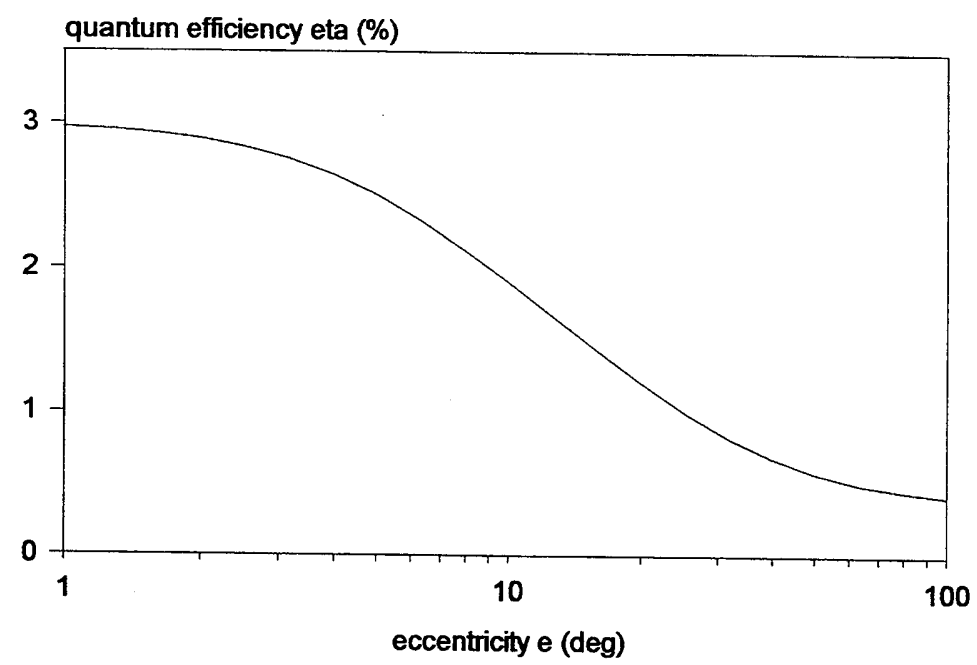

Figure 4.7: Photopic quantum efficiency as a function of retinal eccentricity calculated with Eq. (4.17) and assuming a quantum efficiency of $3 \%$ in the centre of the retina. 


$$
\eta(e)=\eta\left(\frac{0.4}{1+(e / 7)^{2}}+\frac{0.48}{1+(e / 20)^{2}}+0.12\right)
$$

where $\eta$ at the right-hand side of this expression is the quantum efficiency at foveal vision and $e$ is the eccentricity in degrees. Fig. 4.7 shows a plot of this function for the typical situation that the quantum efficiency at foveal vision is $3 \%$.

\subsubsection{Effect of eccentricity on the maximum of the integration area}

As was mentioned in section 2.4 of Chapter 2 , the area where a signal can be compared with noise, is limited by a maximum angular size $X_{\max }$ and by a maximum number of cycles $N_{\max }$. For foveal vision $X_{\max }$ is $12^{\circ}$. For extra-foveal vision, it may be assumed that at an increase of eccentricity, about a constant number of neurons is involved in the integration process. This does not mean that the maximum size of the integration area increases proportionally with the distance between the ganglion cells at increasing eccentricity. A proportional increase could be true for a small part of the area, but is not true for the maximum area as a whole. This is caused by the influence of the high ganglion cell density in the foveal area. At foveal vision, the foveal area is only a small part of the total integration area of $12^{\circ} \times 12^{\circ}$. This makes that for extrafoveal vision the maximum size of the integration area increases much slower than proportionally to the ganglion cell distance in the centre of the object. In many experiments the object field is much smaller than $12^{\circ} \times 12^{\circ}$. For these experiments an increase of $X_{\max }$ at larger eccentricities is irrelevant. From some experiments given in section 4.4 of this chapter, where a large field size was used, it appeared that the variation of $X_{\max }$ with eccentricity can approximately be described by the following expression:

$$
X_{\max }(e)=Y_{\max }(e)=X_{\max }\left(\frac{0.85}{1+(e / 4)^{2}}+\frac{0.15}{1+(e / 12)^{2}}\right)^{-0.5}
$$

where $X_{\max }$ at the right-hand side of this expression is the value of $X_{\max }$ at foveal vision, given in the previous chapter, and $e$ is the eccentricity in degrees.

In section 2.4 of Chapter 2 , it was already mentioned that the limit of the integration area formed by a maximum number of cycles is probably caused by the decrease of contrast sensitivity with increasing distance from the centre of the retina. From measurements by Robson \& Graham (1981, Fig. 3) with strip sized objects, it can be seen that for an eccentric object no limitation of the number of cycles occurs in tangential direction with respect to the centre of the retina. For an object observed in the centre of the retina all dimensions are radial. However, for an eccentric object a distinction has to be made between radial and tangential dimensions. This means that for extrafoveal vision, the last term at the right-hand side of Eqs. (2.48) and 
(2.49) given in Chapter 2 has to be omitted for the tangential direction. For the radial direction, it should be taken into account that the edge of the integration area of an object in the centre of the retina is located at a distance of $1 / 2 N_{\max }$ cycles from the centre. This means that for the maximum number of cycles in radial direction for an eccentric object not $N_{\max }$ should be used but $1 / 2 N_{\max }$. For an eccentric object with radial size $X$ and tangential size $Y$, therefore, the following modifications of Eqs. (2.48) and (2.49) have to be used:

and

$$
X=\left(\frac{1}{X_{0}^{2}}+\frac{1}{X_{\max }^{2}}+\frac{u^{2}}{\left(1 / 2 N_{\max }\right)^{2}}\right)^{-0.5}
$$

$$
Y=\left(\frac{1}{Y_{0}^{2}}+\frac{1}{Y_{\max }^{2}}\right)^{-0.5}
$$

For eccentricities smaller than half the size of the object these formulae cannot be used. To arrive at expressions that can be used for all eccentricities, these equations can further be modified into

and

$$
X=\left(\frac{1}{X_{0}^{2}}+\frac{1}{X_{\max }^{2}}+\frac{\left(1 / 2 X_{\mathrm{o}}\right)^{2}+4 e^{2}}{\left(1 / 2 X_{\mathrm{o}}\right)^{2}+e^{2}} \frac{u^{2}}{N_{\max }^{2}}\right)^{-0.5}
$$

$$
Y=\left(\frac{1}{Y_{0}^{2}}+\frac{1}{Y_{\max }^{2}}+\frac{\left(1 / 2 X_{0}\right)^{2}}{\left(1 / 2 X_{0}\right)^{2}+e^{2}} \frac{u^{2}}{N_{\max }^{2}}\right)^{-0.5}
$$

where by the introduction of an additional factor in the last term a smooth transition is obtained from centric to eccentric vision.

\subsection{Comparison with measurements}

With the formulae given in the preceding section, the constants of the foveal contrast sensitivity model given in section 3.8 of Chapter 3 can be adapted for extra-foveal vision. In this section published contrast sensitivity measurements made at extrafoveal vision will be compared with the so extended model. The measurements are given in chronological order of publication. The density of the on-centre $M$ ganglion cells used in the calculations will be adapted for each eccentricity of the object to obtain a best fit with the high spatial frequency part of the measurements. The so obtained ganglion cell densities will be shown as a function of eccentricity in Fig. 4.15 
at the end of this section in comparison with a curve for the average of the anatomical measurements by Curcio and Allen showed in Fig. 4.5. In the same way as in the preceding chapter, the foveal value of the constants $\sigma_{0}, \eta$, and $k$ will be adapted to the measurements by a simultaneous fit for all eccentricities. This fit will be made by trial and error. If the contrast sensitivity is determined with the aid of a 2AFC method where the results do not correspond with $75 \%$ correct response, $k$ will be corrected with Eq. (2.14) given in Chapter 2. A survey of the values used for $\sigma_{0}, \eta$, and $k$ will be given in Table 4.1 at the end of this section.

\subsubsection{Measurements by Virsu and Rovamo}

In the paper by Virsu \& Rovamo (1979) that was already mentioned in section 3.9.10 of the previous chapter, they published also measurements of the contrast sensitivity function at different retinal eccentricities. The test object was a vertical or horizontal sinusoidal grating pattern generated on the screen of a high resolution monitor provided with a white phosphor (P4). The luminance of the test object was $10 \mathrm{~cd} / \mathrm{m}^{2}$ and the luminance of the surrounding area was the same. The shape of the grating pattern used in one experiment was circular with a constant angular size of $5^{\circ}$. In this experiment, eccentricity was varied along the nasal half of the horizontal meridian of the retina. Fixation was made with both eyes, but the stimulus field was observed with only one eye (the right eye) with a natural pupil. The viewing distance

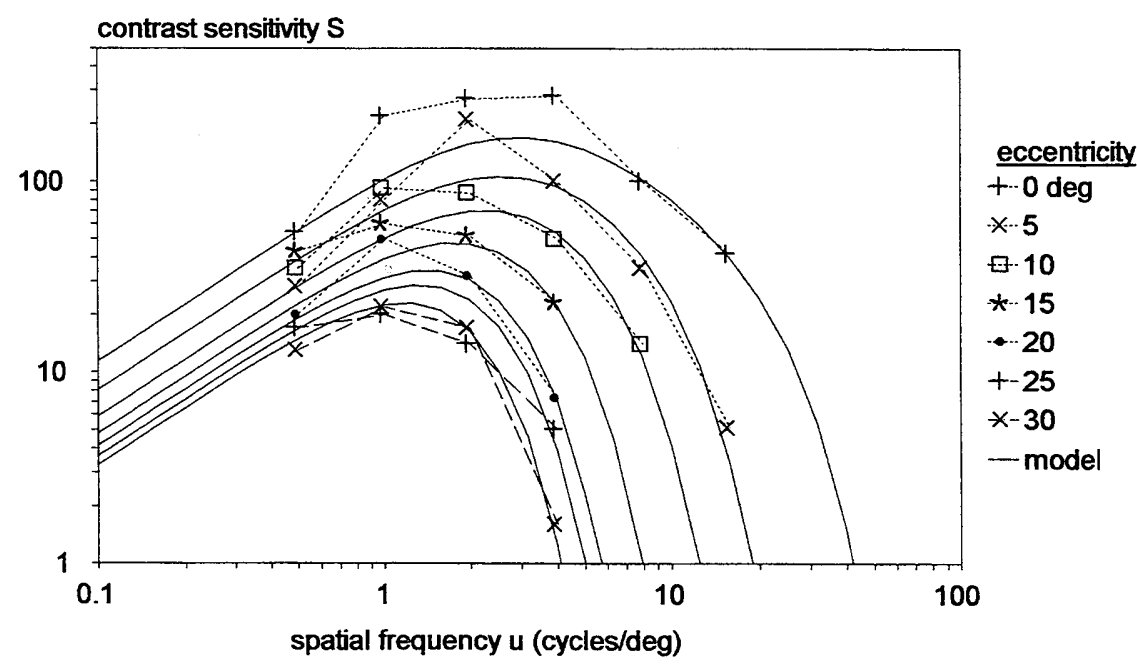

Figure 4.8: Contrast sensitivity function measured by Virsu \& Rovamo (1979) at different eccentricities along the nasal half of the horizontal meridean of the retina. Luminance $10 \mathrm{~cd} / \mathrm{m}^{2}$. Circular field with a diameter of $5^{\circ}$. Monocular viewing with a natural pupil. The solid curves have been calculated with the extended model given in this chapter. 
was $2.28 \mathrm{~m}$. The modulation threshold was determined with the aid of a twoalternative forced-choice method where the threshold corresponded with $84 \%$ correct response. Seven subjects between 25 and 36 years of age participated in the experiments. The reported data are from one subject (VV). The here used measurements were measurements where the orientation of the grating had to be detected.

Measurements and calculations are shown in Fig. 4.8. The values of $\sigma_{0}, \eta$, and $k$ used for the calculation were 0.48 arc $\min , 4.0 \%$, and 4.3 , respectively, after correcting $k$ for the difference between $84 \%$ and $75 \%$ correct response with the aid of Eq. (2.14). Apart from some peaking at intermediate spatial frequencies the general agreement between measurements and calculations is good.

In a second experiment the eccentricity was varied along the superior half of the vertical meridian of the retina. In this experiment the measurements were made with a semicircular grating of which the straight edge was opposing the fixation mark. The radius was $1^{\circ}$ for zero eccentricity and increased with the eccentricity up to $15.8^{\circ}$ in a part of the measurements. This variation was made by changing the viewing distance, starting with a viewing distance of $4.58 \mathrm{~m}$ for zero eccentricity. The eccentricities mentioned by the authors refer to the position of the straight edge of the semicircular pattern that was nearest to the centre of the retina. This has been taken into account in the calculations by adding half the effective radial field size to the mentioned eccentricity. Other conditions were the same as in the first experi-

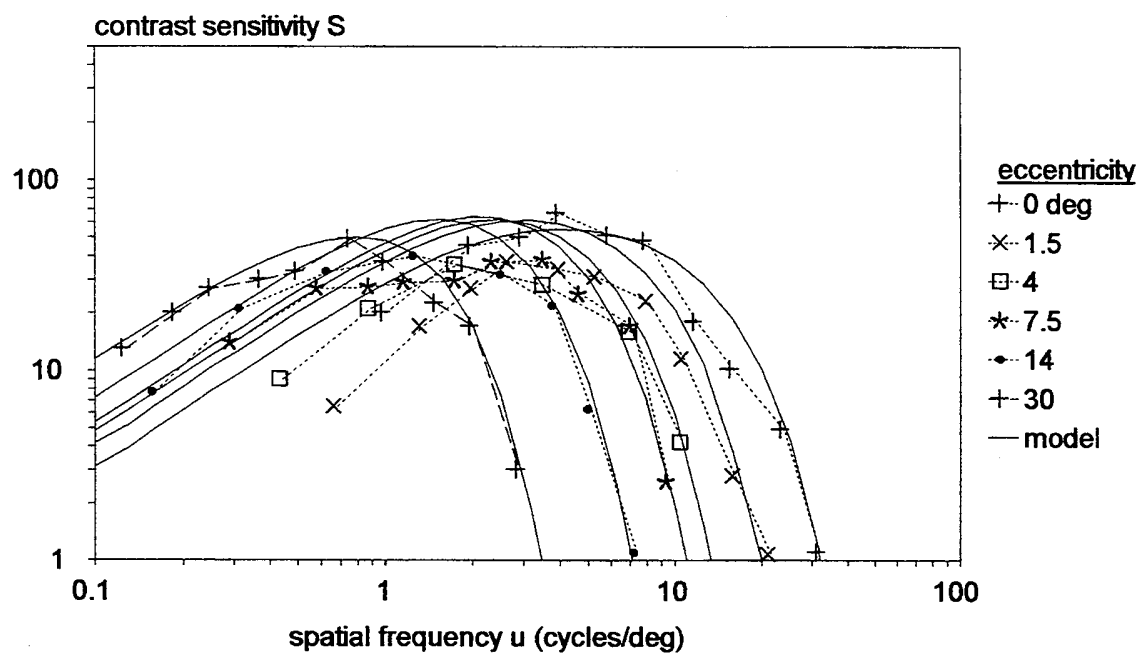

Figure 4.9: Contrast sensitivity function measured by Virsu \& Rovamo (1979) at different eccentricities along the superior half of the vertical meridian of the retina. Luminance $10 \mathrm{~cd} / \mathrm{m}^{2}$. Semicircular field with a radius increasing with eccentricity. Monocular viewing with a natural pupil. The solid curves have been calculated with the extended model given in this chapter. 
ment. The here used measurements are from observer PL for the situation where the field size varied with eccentricity.

Measurements and calculations are shown in Fig. 4.9. The values of $\sigma_{0}, \eta$, and $k$ used for the calculation were 0.68 arc $\min , 3.0 \%$, and 4.7 , respectively, after correcting $k$ for the difference between $84 \%$ and $75 \%$ correct response with the aid of Eq. (2.14). Compared with Fig. 4.7, the curves for the high eccentricities clearly show the effect of the simultaneously increased field size. This overcompensates the drop of contrast sensitivity at low spatial frequencies and makes that the curves as a whole shift to lower spatial frequencies. Apart from some deviations at intermediate eccentricities, the calculated curves show a good agreement with the measurements.

\subsubsection{Measurements by Robson and Graham}

Robson \& Graham (1981) measured the contrast sensitivity at different spatial frequencies as a function of eccentricity. The measurements were made along both halves of the vertical meridian of the retina. The test object was a horizontally oriented sinusoidal grating pattern generated on the screen of a CRT provided with P31 phosphor. The luminance of the test object was $500 \mathrm{~cd} / \mathrm{m}^{2}$ and the surrounding was kept on the same luminance. The test object contained four cycles and had a square shape without sharp edges. The spatial frequency on the display was fixed at $3 \mathrm{cycles} / \mathrm{cm}$ and the angular spatial frequency for the observer was varied by varying the viewing distance. For 3 cycles $/ \mathrm{deg}$, the viewing distance was $0.57 \mathrm{~m}$. The eccentricity was expressed in the number of cycles of the concerning spatial frequency, as they wanted to investigate the effect of the number of cycles of the eccentricity on the contrast sensitivity. The pattern on the display was turned on and off with a Gaussian temporal function lasting $167 \mathrm{msec}$ above half its peak value. The test object was observed with both eyes and with a natural pupil. The observer had to fixate midway between two horizontally separated dark fixation marks. The modulation threshold was determined with the aid of a temporal two-alternative forcedchoice method where the threshold corresponded with $90 \%$ correct response. The authors served as subject. The reported data are from one subject: JR. Here the average of the measurements for the two halves of the vertical meridian is used.

Measurements and calculations are shown in Fig. 4.10. The values of $\sigma_{0}, \eta$, and $k$ used for the calculation were 0.67 arc $\mathrm{min}, 3.0 \%$, and 4.9 , respectively, after correcting $k$ for the difference between $90 \%$ and $75 \%$ correct response with Eq. (2.14). The values of $N_{g}$ have here been calculated with Eq. (4.8) using for $e_{\mathrm{g}}$ a value of $3.9^{\circ}$. Note that the eccentricity is expressed in the number of cycles of the concerning spatial frequency and varies, therefore, along the curves (except for the curve at zero eccentricity). The somewhat flattened shape of the curves at small eccentricities is caused by using a fixed number of cycles, which reduces the angular 


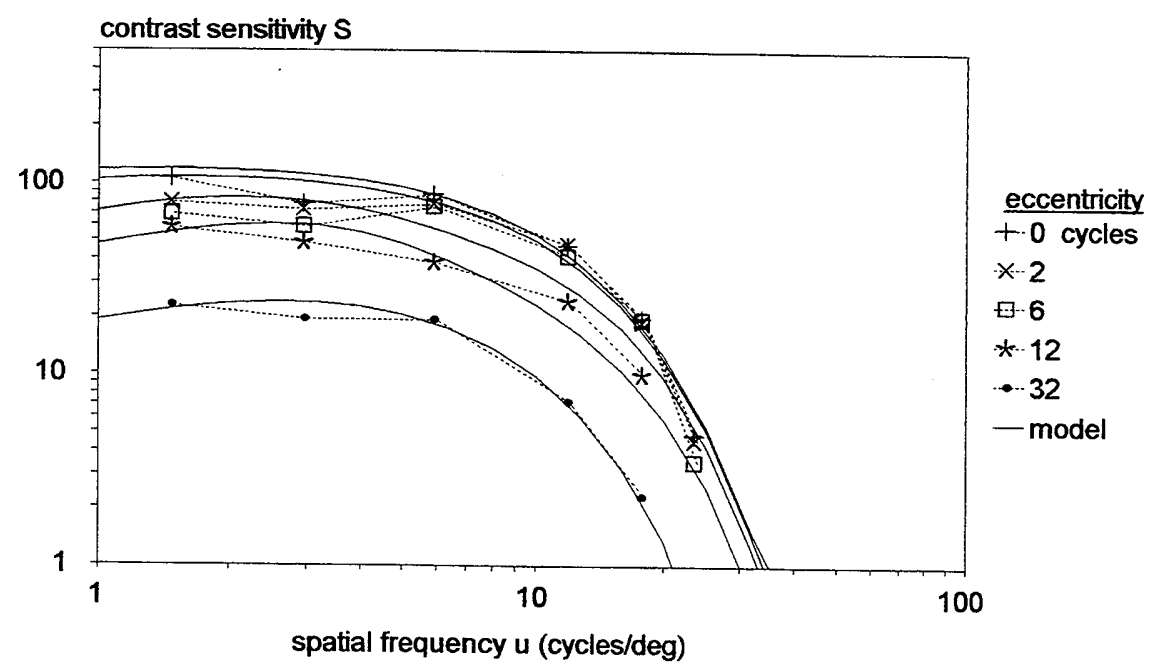

Figure 4.10: Contrast sensitivity function measured by Robson \& Graham (1981) at different eccentricities along the vertical meridian of the retina. The eccentricity is varied with the number of cycles of the concerning spatial frequency and varies, therefore, along the curves (except for that at zero eccentricity). Square test pattern consisting of 4 cycles. Luminance $500 \mathrm{~cd} / \mathrm{m}^{2}$. Binocular viewing with a natural pupil. The solid curves have been calculated with the extended model given in this chapter.

field size at increasing spatial frequency. This is similar as the flattening of the contrast sensitivity curve shown in Fig. 3.17 of Chapter 3, which is due to the same effect. Apart from a remarkable dip at a spatial frequency of 3 cycles/deg, the measurements are in reasonable agreement with the measurements.

Fig 4.11 shows the same measurements and calculations, but now plotted as a function of the eccentricity expressed in the number of cycles of the concerning frequency with the spatial frequency as parameter. From the figure, it can be seen that the measurements show here a nearly constant logarithmic decrease of contrast sensitivity with eccentricity, which is approximately equal for all spatial frequencies. This decrease is not influenced by limitations formed by the size of the object, as the test object contained only four cycles and its angular size was not larger than $2.7^{\circ}$ for the lowest spatial frequency. In section 2.4 of Chapter 2 , it has already been mentioned that the limitation of the integration area by a fixed number of cycles is probably caused by the decrease of contrast sensitivity with increasing distance from the centre of the retina. The approximately equal decrease of contrast sensitivity as a function of the number of cycles shown in Fig. 4.11 supports the idea that the integration limit formed by a fixed number of cycles is in fact caused by an equal decrease of contrast sensitivity for all spatial frequencies at an increase of the eccentricity with an equal number of cycles. At foveal vision, the limit of 15 cycles for the visual angle of an object corresponds with 7.5 cycles over its radial dimensions. 


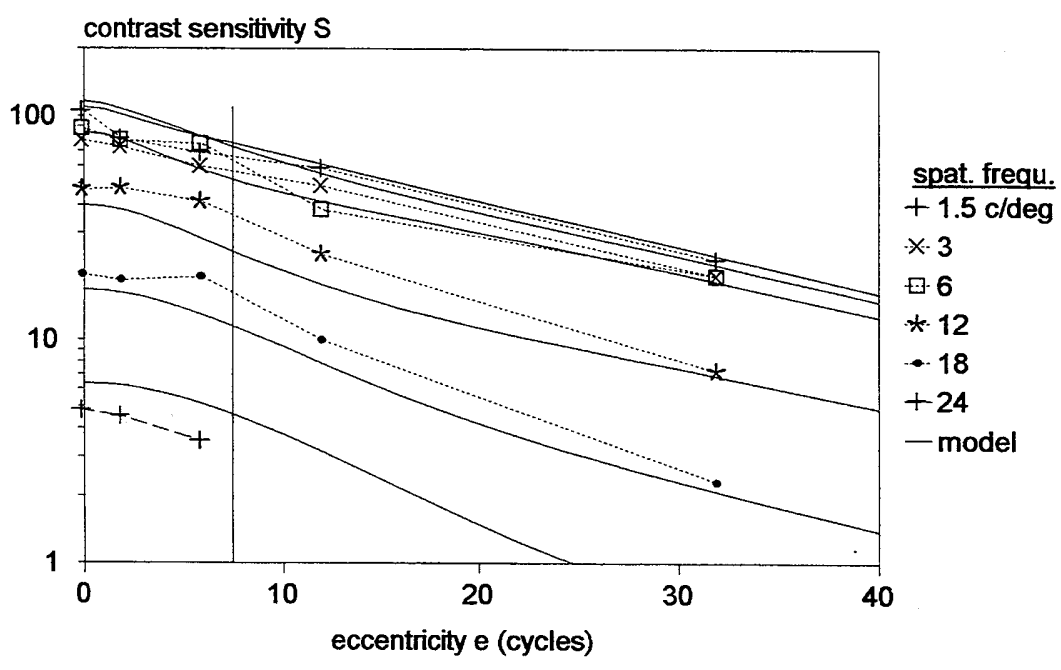

Figure 4.1 l: Same data as Fig. 4.10, but plotted as a function of the eccentricity expressed in the number of cycles of the concerning spatial frequency. The vertical line at an eccentricity of 7.5 cycles indicates the integration limit at foveal vision.

This limit is shown in Fig. 4.11 by the vertical line at an eccentricity of 7.5 cycles. At this eccentricity, the contrast sensitivity has decreased with a factor of about 0.65 for all spatial frequencies. Areas with lower contrast sensitivity do probably not contribute anymore to the integration process.

\subsubsection{Measurements by Kelly}

Kelly (1984) measured the contrast sensitivity function at different eccentricities using contiguous annular zones as stimulus. The stimuli consisted of radial grating patterns generated on the screen of a colour CRT used in white mode. The annular zones had outside diameters of $4^{\circ}, 8^{\circ}, 16^{\circ}$, and $30^{\circ}$. The outside diameter of each zone coincided with the inner diameter of the neighbouring zone. The eccentricity was defined as the average of the inner and outer radius, except for the innermost area where the eccentricity was zero. The pattern was simultaneously varied in counter phase with a temporal sinusoidal variation of $0.5 \mathrm{~Hz}$. This frequency may be assumed to be low enough to consider the measurements as static. See Chapter 5 . The luminance was not mentioned, but was assumed to be $10 \mathrm{~cd} / \mathrm{m}^{2}$ for the calculations, as most probable value. The object had a constant size and the eccentricity and the angular size of the pattern were, therefore, varied together by varying the viewing distance. The subject fixated a small dot in the centre of the stimulus pattern and this pattern was stabilized on the retina by special equipment. Viewing was monocular with an artificial pupil of $3 \mathrm{~mm}$. The modulation threshold was determined by the 


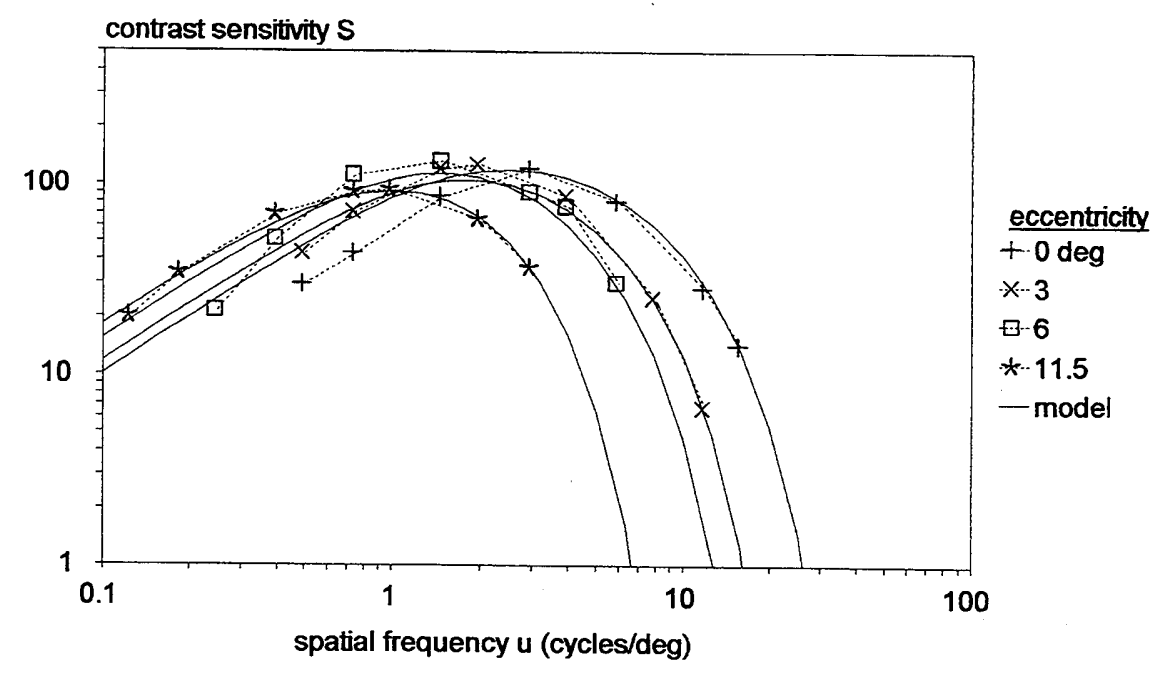

Figure 4.12: Contrast sensitivity function at different eccentricities measured by Kelly (1984) for neighbouring annular zones. Monocular viewing with an artificial pupil of 3 $\mathrm{mm}$. The solid curves have been calculated with the extended model given in this chapter.

method of adjustment. The reported data are from one subject: the author.

Measurements and calculations are shown in Fig. 4.12. The values of $\sigma_{0}, \eta$, and $k$ used for the calculation were 0.9 arc $\min , 2.5 \%$, and 4.1 , respectively. The general agreement between measurements and calculations is good.

\subsubsection{Measurements by Mayer and Tyler}

Mayer \& Tyler (1986) measured the contrast sensitivity function at zero eccentricity and at an eccentricity of $3.5^{\circ}$ along the inferior vertical meridian of the retina. The test object was a vertically oriented sinusoidal grating pattern generated on a monitor screen provided with P31 phosphor. The luminance was $40 \mathrm{~cd} / \mathrm{m}^{2}$. The angular width of the pattern was $4^{\circ}$ and the angular height was $1^{\circ}$. The pattern was surrounded by a cardboard matched in colour and luminance with the grating. Observers viewed the display with both eyes and with a natural pupil. The viewing distance was $1.52 \mathrm{~m}$. The modulation threshold was determined by a Weibull function used as approximation of the psychometric function (See section 2.2 of Chapter 2). The quantity $\alpha$ of this function was used as modulation threshold. Three subjects participated in the experiments. Here the data from one subject, the first author, are used, being the only subject for which foveal and extrafoveal data were reported.

Measurements and calculations are shown in Fig. 4.13. The values of $\sigma_{0}, \eta$, and 


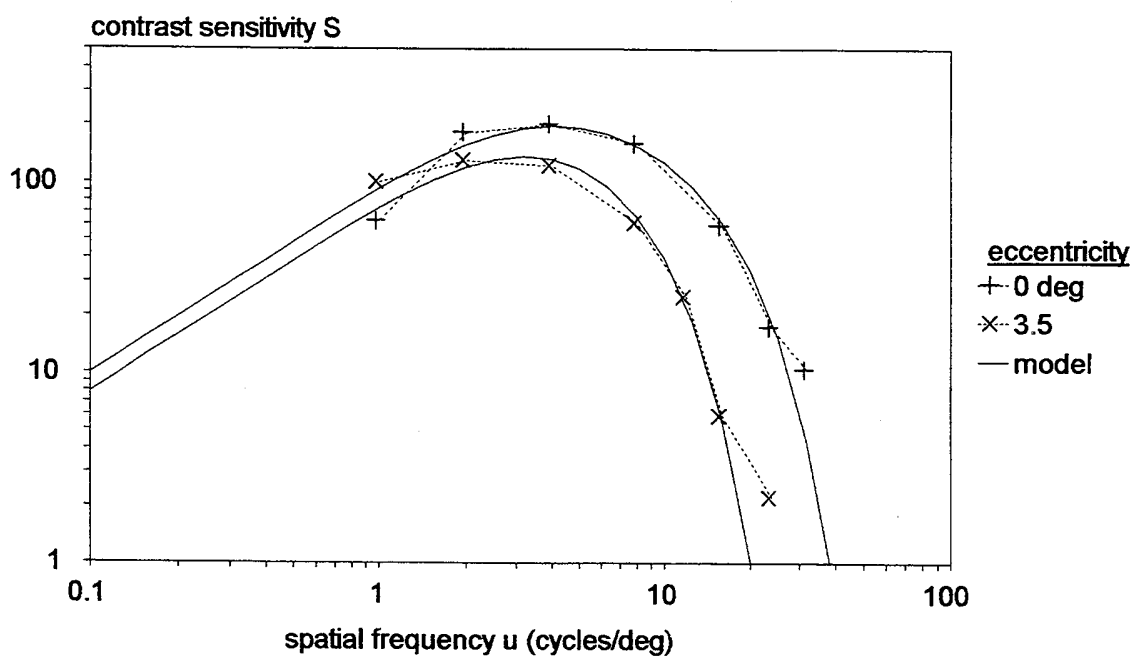

Figure 4.13: Contrast sensitivity function measured by Mayer and Tyler (1986) at zero eccentricity and at an eccentricity of $3.5^{\circ}$ along the inferior half of the vertical meridian of the retina. Luminance $40 \mathrm{~cd} / \mathrm{m}^{2}$. Rectangular field with a width of $4^{\circ}$ and a height of $1^{\circ}$. Binocular viewing with a natural pupil. The solid curves have been calculated with the extended model given in this chapter.

$k$ used for the calculation were 0.66 arc $\min , 2.2 \%$, and 3.3, respectively, after correcting $k$ for the quantity $\alpha$ of the Weibull function with the aid of Eqs. (2.7) and (2.10). The agreement between measurements and calculations is very good, apart from a strange crossing of the measured curves at a spatial frequency of about one cycle/deg and a strange deviation of these curves at the highest spatial frequency.

\subsubsection{Measurements by Johnston}

Johnston (1987) measured the contrast sensitivity function at different eccentricities in the nasal visual field. This field corresponds with the temporal half along the horizontal meridian of the retina. An emphasis was made on the high-frequency part of the contrast sensitivity curve. The test object was a vertical or horizontal sinusoidal grating pattern generated on the screen of an oscilloscope provided with bluegreen P31 phosphor. The luminance was $10 \mathrm{~cd} / \mathrm{m}^{2}$. The surrounding of the pattern was masked with a white card with a luminance matched with the display. The pattern contained 12 cycles. It was square and had a constant size of $5.2 \mathrm{~cm}$. The spatial frequency was varied by varying the viewing distance. In this way, the angular field size varied inversely proportionally with the spatial frequency. At 12 cycles/deg the viewing distance was $3 \mathrm{~m}$ and the angular size was $1^{\circ}$. Fixation was made with a fixation spot. Viewing was monocular with a natural pupil. The modulation threshold was determined with the aid of a temporal two-alternative forced-choice method. The 


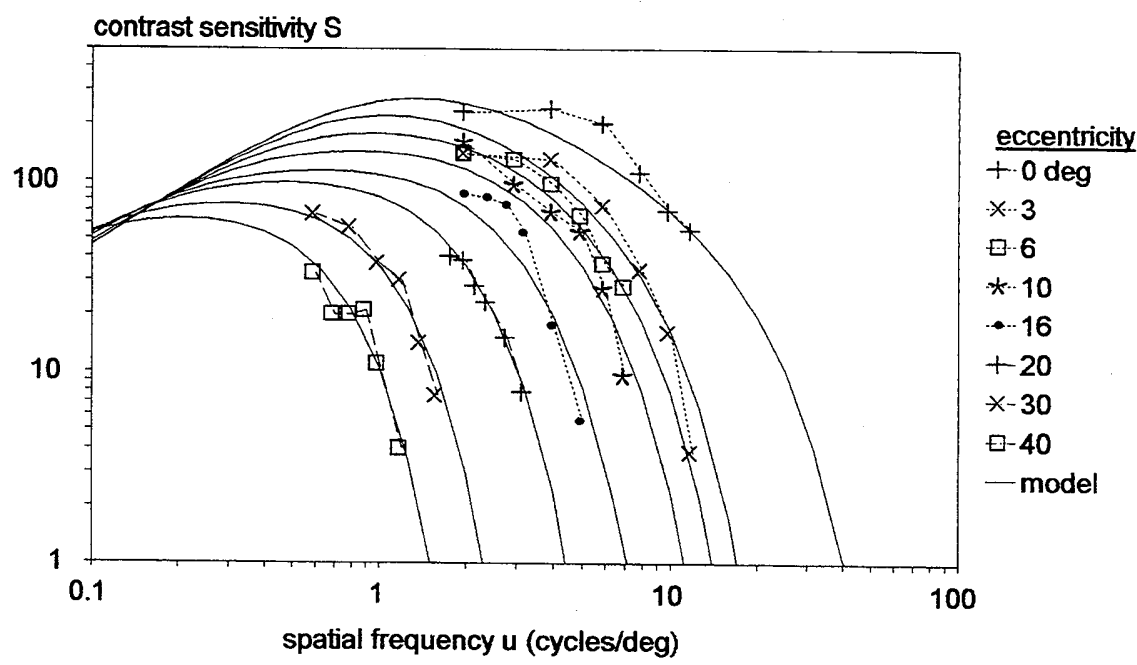

Figure 4.14: Contrast sensitivity function measured Johnston (1987) at different eccentricities along the temporal half of the horizontal meridian of the retina. Luminance $10 \mathrm{~cd} / \mathrm{m}^{2}$. Square field with a size inversely proportional to the spatial frequency. Monocular viewing with a natural pupil. The solid curves have been calculated with the extended model given in this chapter.

here used data are from one subject, the author, for vertically oriented gratings.

Measurements and calculations are shown in Fig. 4.14.The values of $\sigma_{0}, \eta$, and $k$ used for the calculation were $0.5 \mathrm{arc} \min , 3.5 \%$, and 3.4 , respectively. The agreement between measurements and calculations is good.

\subsubsection{Measurements by Pointer and Hess}

Pointer and Hess (1989) measured the contrast sensitivity function at different eccentricities along the horizontal and vertical meridian of the retina with special emphasis on the low spatial frequency range. The test object was a horizontally oriented sinusoidal grating pattern generated on the screen of a CRT monitor provided with a white phosphor $(\mathrm{P} 4)$. The luminance was $100 \mathrm{~cd} / \mathrm{m}^{2}$. The display screen was surrounded by a field with the same luminance. The pattern was circular with a circularly-symmetric two-dimensional Gaussian envelope and a Gaussian time profile. The spatial and temporal Gaussian windows were truncated at their $1 / e$ value. The spatial size was 6.4 cycles and the temporal duration was $500 \mathrm{msec}$. The spatial frequency was varied by varying the viewing distance between 0.18 and $3.7 \mathrm{~m}$. In this way, the angular field size varied inversely proportionally with the spatial frequency. The spatial luminance patterns were presented with a temporal frequency of $1 \mathrm{~Hz}$ in counter phase. This frequency may be assumed to be low enough to consider the 


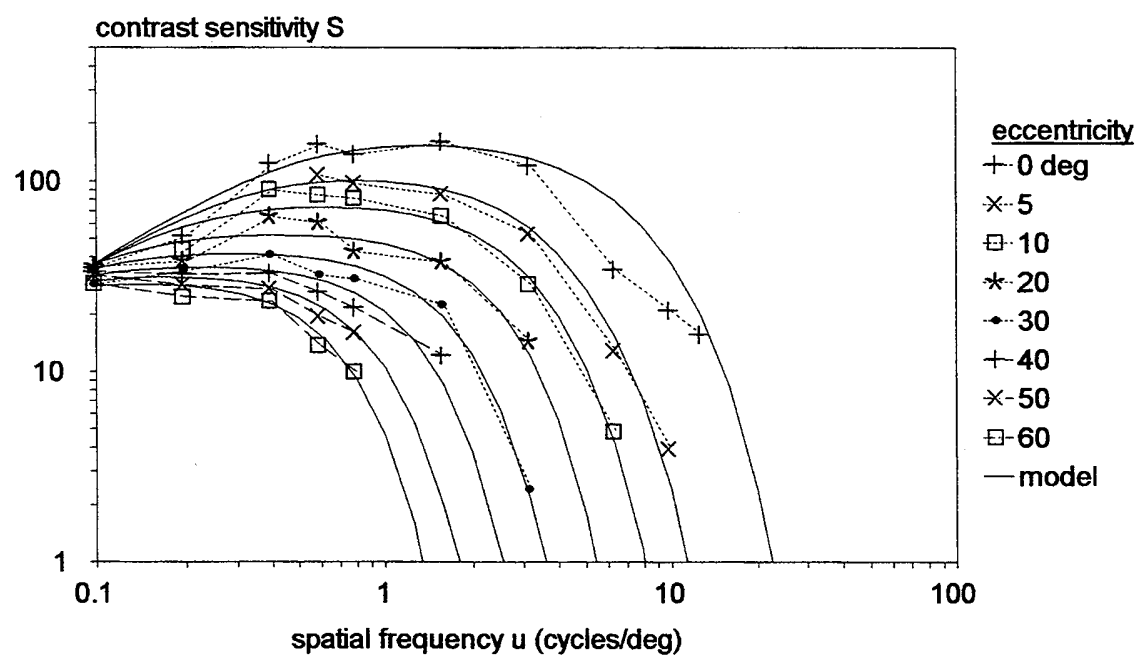

Figure 4.15: Contrast sensitivity function measured Pointer \& Hess (1989) at different eccentricities along the horizontal meridian of the retina. Luminance $100 \mathrm{~cd} / \mathrm{m}^{2}$. Circular field with a size inversely proportional with spatial frequency. Monocular viewing with a natural pupil. The solid curves have been calculated with the extended model given in this chapter.

measurements as static. See Chapter 5. Fixation was made with a fixation light outside the centre of the display. The test object was always in the centre of the display. Viewing was monocular with the observer's dominant eye and with a natural pupil. The modulation threshold was determined with the aid of a temporal twoalternative forced-choice method. Three subjects participated in the experiments. The here used data are the measurement results for the horizontal meridian from two subjects (PAB and JSP, the first author) averaged over subjects and over both halves of the meridian.

Measurements and calculations are shown in Fig. 4.15. The values of $\sigma_{0}, \eta$, and $k$ used for the calculation were 1.1 arc $\mathrm{min}, 3.0 \%$, and 4.1 , respectively. Apart from the curve at zero eccentricity, the calculated curves are in very good agreement with the measurements. The shape of the curves in this figure and in Fig. 4.13 show a resemblance with a bird's head with a sharp beak. This is caused by the increase of the angular field size at low spatial frequencies. In Fig. 4.13 no measurements are available in this part of the curves. However, the measurements for this area shown in Fig. 4.14 confirm very well the predictions by the model. 


\subsubsection{Survey of the measurements}

For the given measurements, the densities of the on-centre $M$ ganglion cells used in the calculations were adapted for each eccentricity to obtain a best fit with the high spatial frequency part of the measurements. The so obtained densities of the oncentre $M$ ganglion cells are shown in Fig. 4.16 as a function of eccentricity. This figure also shows the average ganglion cell density derived from the anatomical measurements by Curcio and Allen given in Fig. 4.5, after multiplication of the density with 0.05 because of the assumption that $5 \%$ of the ganglion cells are oncentre $M$ cells. Because of the spread of the data, it appeared to have no sense to compare the data separately with the measurements for each of the concerning areas on the retina. The figure shows a general agreement between the ganglion cell density derived from the various contrast sensitivity measurements. There is also some agreement with the average density obtained from the anatomical data. However, at higher eccentricities, the density obtained from these data is generally lower than that derived from the contrast sensitivity measurements. This could mean that the assumed constant value of $5 \%$ for the on-centre $M$ cells is in reality closer to 7 or $10 \%$ at these eccentricities.

A survey of the values of $\sigma_{0}, \eta$, and $k$ used for the evaluation of the measurements is given in Table 4.1. The values for $\eta$ are close to $3 \%$, but the values for $\sigma_{0}$ and $k$ are generally somewhat higher than the data given in Table 3.1 of Chapter 3 .

Table 4.1: $\sigma_{0}, \eta$, and $k$ values used for the evaluation of the measurements

\begin{tabular}{|l|c|c|c|}
\hline \multicolumn{1}{|c|}{ author } & $\begin{array}{c}\boldsymbol{\sigma}_{0} \\
(\mathrm{arc} \mathrm{min})\end{array}$ & $\begin{array}{c}\eta \\
(\%)\end{array}$ & $\boldsymbol{k}$ \\
\hline Virsu \& Rovamo (1979) & 0.48 & 4.0 & 4.3 \\
& 0.68 & 3.0 & 4.7 \\
\hline Robson \& Graham (1981) & 0.67 & 3.0 & 4.9 \\
\hline Kelly (1984) & 0.9 & 2.5 & 4.4 \\
\hline Mayer \& Tyler (1986) & 0.66 & 2.2 & 3.3 \\
\hline Johnston (1987) & 0.5 & 3.5 & 3.4 \\
\hline Pointer \&Hess (1989) & 1.1 & 3.0 & 4.1 \\
\hline
\end{tabular}




\subsection{Summary and conclusions}

In this chapter the spatial contrast sensitivity model given in the previous chapter has been extended to extra-foveal vision. For this purpose relations have been developed for the dependence of the constants used in this model on retinal eccentricity. For these relations, use has been made of anatomical and biological data of the density distribution of different retinal cell types. From this analysis, it appeared that especially the density variation of the on-centre $M$ ganglion cells has a large effect on the extra-foveal contrast sensitivity. With the aid of the given relations for the contrast sensitivity, it appears that the density of these cells as a function of eccentricity can even be derived from measurements of the contrast sensitivity function at different eccentricities.

The extended model showed a good agreement with various published measurements of extra-foveal contrast sensitivity. This was, of course, also partly due to the many assumptions made in the model, which were based on results obtained with these measurements. However, the agreement obtained with these assumptions for the different types of measurements shows that these assumptions have more general validity.

The density of the on-centre $M$ ganglion cells that could be derived from the contrast sensitivity measurements with the aid of the given expressions appeared largely to agree with measurements of anatomical data. However, at larger eccentricities, the calculated densities were somewhat higher than the densities derived from the anatomical data. This could mean that the assumed constant value of $5 \%$ for the part of on-centre $M$ cells of the total number of ganglion cells is in reality closer to 7 or $10 \%$ at these eccentricities.

Measurements by Robson \& Graham (1981) confirmed the presumption, made in section 2.4 of Chapter 2, that the limit of the integration area by a fixed number of cycles is probably caused by a decrease of contrast sensitivity with increasing eccentricity. These measurements showed that this decrease is approximately equal for all spatial frequencies at an increase of the eccentricity with an equal number of cycles. From measurements by Robson and Graham, it also appears that this limit occurs only in radial direction. At foveal vision all dimensions are radial so that this limit is always present, but at eccentric vision a distinction has to be made between radial and tangential dimensions of the object with respect to the centre of the retina. 


\section{References}

Coletta, N.J. \& Williams, D.R (1987). Psychophysical estimate of extrafoveal cone spacing. Journal of the Optical Society of America A, 4, 1503-1513.

Curcio, C.A. \& Allen, K.A. (1990). Topography of ganglion cells in human retina. Journal of Comparative Neurology, 300, 5-25.

Curcio, C.A., Sloan, K.R, Packer, O., Hendrickson, A.E., and Kalina, R.E. (1987). Distribution of cones in human and monkey retina: individual variability and radial asymmetry. Science, 236, 579-582.

Henry, G.H. \& Vidyasagar, T.R (1991). Evaluation of mammalian visual pathways. In: J.R. Cronly-Dillon \& R.L. Gregory (Eds.) Vision and visual dysfunction, II. Evolution of the eye and visual system, Chapter 20. MacMillan Press, London.

Johnston, A. (1987). Spatial scaling of central and peripheral contrast-sensitivity functions. Journal of the Optical Society of America A, 4, 1583-1593.

Kelly, D.H. (1984). Retinal inhomogeneity. I. Spatiotemporal contrast sensitivity. Journal of the Optical Society of America A, 1, 107-1 13.

Lee, B.B. (1996). Receptive field structure in the primate retina. Vision Research, 36, 631-644.

Mayer, M.J. \& Tyler, C.W. (1986). Invariance of the slope of the psychometric function with spatial summation. Journal of the Optical Society of America A, 3, 1166-11 172.

Østerberg, G. (1935). Topography of the layer of rods and cones in the human retina. Acta Ophthalmologica. Suppl., 6, 1-103.

Pointer, J.S. \& Hess, R.F. (1989). The contrast sensitivity gradient across the human visual field: with emphasis on the low spatial frequency range. Vision Research, 29, 1133-1151.

Polyak, S. (1957). The vertebrate visual system. University of Chicago Press, Chicago.

Robson, J.G. \& Graham, N. (1981). Probability summation and regional variation in contrast sensitivity across the visual field. Vision Research, 21, 409-418.

Virsu, V. \& Rovamo, J. (1979). Visual resolution, contrast sensitivity, and the cortical magnification factor. Experimental Brain Research, 37, 475-494.

Wässle, H., Grünert, U., Röhrenbeck, J., and Boycott, B.B. (1990). Retinal ganglion cell density and cortical magnification factor in the primate. Vision Research, 30, 1897-1911.

Williams, D.R., (1988) Topography of the foveal cone mosaic in the living human eye. Vision Research, 28, 433-454. 


\section{Chapter 5}

\section{Extension of the contrast sensitivity model to the temporal domain}

\subsection{Introduction}

The contrast sensitivity model given in Chapter 3 was restricted to spatial contrast sensitivity. In this chapter the model will be extended to the temporal domain so that a spatiotemporal model is obtained that can also be used for purely temporal luminance variations. Temporal contrast sensitivity has already intensively been studied in the fifties by de Lange $(1952,1954,1957,1958 \mathrm{a}, 1958 \mathrm{~b}, 1961)$ and in the sixties and seventies by Kelly $(1960,1961,1971,1972,1979)$ and Roufs (1972a, 1972b, 1973, 1974a, 1974b, 1974c). Kelly (1960) proposed to combine spatial and temporal contrast sensitivity measurements by using spatiotemporal stimuli to get more insight in spatiotemporal interactions. The two-dimensional contrast sensitivity function obtained with these types of stimuli is not simply the product of a spatial and a temporal response, but shows a much more complicated behaviour (Robson, 1966; van Nes et al., 1967; Kelly, 1971, 1972, 1979; Koenderink \& van Doorn, 1979). To explain this behaviour, Kulikowski \& Tolhurst (1973) supposed the existence of a sustained and a transient channel in the human visual system, analogous to supposed spatial-frequency channels in the spatial domain.

For the temporal contrast sensitivity, it may be assumed that it is determined by internal noise in the same way as for spatial contrast sensitivity. Besides a spatial character, noise generally has also a temporal character. For the here given spatiotemporal model no different channels will be assumed for a sustained and a transient response but the complicated spatiotemporal behaviour of the visual system will be explained in a much simpler way. In the model is only assumed that the inhibition signal undergoes besides spatial filtering also temporal filtering before being subtracted from the photo-receptor signal in the inhibition process.

This model has some similarity with a model proposed by Burbeck and Kelly (1980), where the spatiotemporal contrast sensitivity function is obtained as the 
difference between an excitatory mechanism and an inhibitory mechanism, and where each of these two responses is the product of an exclusively spatial and an exclusively temporal response curve. The shape of these response curves has to be determined by data fitting. However, the here given model is different from this model and has more resemblance with a model given by Fleet, Hallet and Jepson (1985) where the inhibition signal is subtracted from the photo-receptor signal in a similar way as in the model of Burbeck and Kelly, but where different spatial and temporal functions are used. Also their treatment is different from the treatment that will be used here. Another approach was followed by Watson (1986) in a working model for temporal contrast sensitivity where two temporal response functions are subtracted from each other. However, this model, which is also different from the model that will be given here, does not contain the spatial aspects and it can, therefore, be used only to temporal contrast sensitivity. The model that will be given here is based on some simple assumptions about the temporal effects of the lateral inhibition.

\subsection{Generalization of the contrast sensitivity model}

The basic assumption of the here given spatiotemporal model is, that the inhibition signal undergoes temporal filtering in addition to spatial filtering before being subtracted from the photo-receptor signal. For this purpose, Eq. (3.26) given in Chapter 3 for the spatial contrast sensitivity is modified by introducing a modification of the denominator of the term with $\Phi_{0}$, which represents the squared MTF of the inhibition process (Barten, 1993). As mentioned in section 3.6 of Chapter 3 , the MTF of the lateral inhibition process consists of a highpass filter given by $1-F(u)$, where $F(u)$ is the MTF of the lowpass filter used for the inhibition. The shape of this function is described by Eq. (3.21). For the extension of the spatial contrast sensitivity model to the temporal domain is now assumed that the function $1-F(u)$ has to be replaced by the function

$$
G(u, w)=H_{1}(w)\left\{1-H_{2}(w) F(u)\right\}
$$

where $w$ is the temporal frequency, $H_{1}(w)$ is a function that represents the temporal filtering of the photo-receptor signal on its way from the photo-receptors to the brain and $\mathrm{H}_{2}(w)$ is a function that represents the temporal filtering of the inhibition signal before being subtracted from the photo-receptor signal. Fig 5.1 is a block diagram of the sow obtained processing of information and noise. It is an extension of the block diagram shown in Fig. 3.1.

By replacing the denominator of the term with $\Phi_{0}$ in Eq. (3.26) by $G^{2}(u, w)$ one obtains as the equation for the here proposed spatiotemporal contrast sensitivity function 


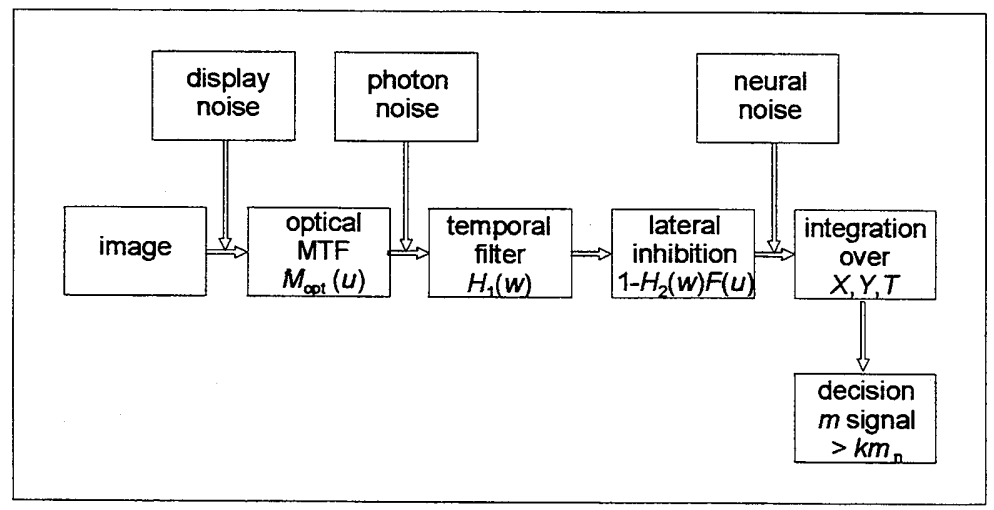

Figure 5.1: Block diagram of the processing of information and noise in the visual system according to the here given spatiotemporal contrast sensitivity model.

$$
S(u, w)=\frac{M_{\mathrm{spat}}(u) / k}{\sqrt{\frac{2}{T}\left(\frac{1}{X_{0}^{2}}+\frac{1}{X_{\max }^{2}}+\frac{u^{2}}{N_{\max }^{2}}\right)\left(\frac{1}{\eta p E}+\frac{\Phi_{0}}{\left[H_{1}(w)\left\{1-H_{2}(w) F(u)\right\}\right]^{2}}\right)}}
$$

where $F(u)$ is given by Eq. (3.21). This equation holds for equal image dimensions in $x$ and $y$ direction. For monocular instead of binocular viewing, the factor of two under the square-root sign has to be replaced by four. The temporal functions $H_{1}(w)$ and $\mathrm{H}_{2}(w)$ will be discussed in more detail in the following section.

\subsection{Temporal filter functions}

The functions $H_{1}(w)$ and $H_{2}(w)$ are temporal filter functions that describe the MTF of the temporal impulse response of the eye. According to de Lange (1952) the temporal filtering in the eye can be compared with the filtering by an electrical circuit consisting of several cascaded RC filters. The impulse response of one stage of such a filter is

$$
h(t)=\frac{1}{\tau} \mathrm{e}^{-t / \tau}
$$

where $h(t)=0$ for $t<0$ and $\tau$ is the RC time constant. For a cascade of $n$ such stages with the same RC time, the impulse response is

$$
h(t)=\frac{n(t / \tau)^{n-1}}{n ! \tau} \mathrm{e}^{-t / \tau}
$$


The MTF resulting from this impulse response can be obtained by taking the absolute value of its Fourier transform. This gives for Eq. (5.4)

$$
H(w)=\frac{1}{\left\{1+(2 \pi \tau w)^{2}\right\}^{n / 2}}
$$

It appears that the functions $H_{1}(w)$ and $H_{2}(w)$ can indeed be described by this function with values $\tau_{1}, n_{1}$ and $\tau_{2}, n_{2}$, respectively, for the constants $\tau$ and $n$. This does not necessarily mean that the concerning impulse response is given by Eq. (5.4). As $H_{1}(w)$ and $H_{2}(w)$ contain only amplitude information and no phase information, the concerning impulse response functions cannot be found by an inverse Fourier transform of these functions. The temporal impulse response function that can be obtained with the here given model will further be treated in section 5.9.

For wt»l the function given by Eq. (5.5) has an asymptotic slope $-n$ on double logarithmic scale. From Eq. (5.2), it follows then that contrast sensitivity as a function of temporal frequency will have a decay with a slope $-n_{1}$ on double logarithmic scale at high temporal frequencies. Daly and Normann (1985) measured the electrical response of cones in the eyes of turtles and found a decay corresponding with an asymptotic slope of about -6 . They also mention that similar results have been found by other authors for other animals. Watson (1986) uses a slope -9 in his model. Roufs (1972b) already remarked that the value of $n$ is not very critical for a fit with measured data, because of the spread of the data points, but that the value of $\tau$ depends on the chosen value of $n$. Here a best fit with measured data was obtained with a slope -7 , so that a value 7 will be used for $n_{1}$ in the here given model. For $n_{2}$.a value 4 will be used, but a different value would also have been possible, as most measurements are not very critical for this value.

The values of $n_{1}$ and $n_{2}$ are essentially fixed, because they are determined by the biological structure of the neural cells or by the number of synapses of these cells. Conversely, the time constants $\tau_{1}$ and $\tau_{2}$ depend on retinal illuminance and field size and can also be different for different subjects. In the following sections a comparison of the model will be given with published contrast sensitivity measurements. For measurements with a series of data curves, a simultaneous fit will be made for all curves similarly as in the preceding chapters.

\subsection{Spatiotemporal contrast sensitivity measurements}

The spatiotemporal stimulus used in spatiotemporal contrast sensitivity measurements can generally be written in the form

$$
L(x, t)=\bar{L}\{1+m \cos (2 \pi u x) \cos (2 \pi w t)\}
$$


where $\bar{L}$ is the average luminance and $u$ and $w$ is the spatial and temporal frequency, respectively. Besides this standing wave pattern, a traveling wave pattern is sometimes used. This has the form

$$
L(x, t)=\bar{L}[1+m \cos \{2 \pi u(x-c t)\}]
$$

where $c$ is the velocity of the pattern. With $w=u \times c$ and the use of a well-known trigonometric relation, Eq. (5.6) can be written as

$$
L(x, t)=\bar{L}[1+1 / 2 m \cos \{2 \pi u(x+c t)\}+1 / 2 m \cos \{2 \pi u(x-c t)\}]
$$

This means that a standing wave can be considered as the sum of two traveling waves moving in opposite directions. Therefore, eye movements can form a problem in measuring spatiotemporal contrast sensitivity. Kelly (1979) showed that with special measures for eye stabilization no difference in contrast sensitivity is found between standing waves and traveling waves.

The first combined spatiotemporal contrast sensitivity measurements have been made by Robson (1966). He measured the spatial contrast sensitivity function at four different temporal frequencies and the temporal contrast sensitivity function at four different spatial frequencies. The measurements were made at a luminance of $20 \mathrm{~cd} / \mathrm{m}^{2}$ using a vertically oriented sinusoidal grating pattern generated on the screen of an oscilloscope tube. The angular size of the test object was $2.5^{\circ} \times 2.5^{\circ}$ and the measurements were made at a viewing distance of $1 \mathrm{~m}$. The observer looked at the test object with both eyes and with a natural pupil. The author (IGR), was the observer. The spatial contrast sensitivity measurements at the lowest temporal frequency $(1 \mathrm{~Hz}$ ) have already been mentioned in section 3.9.3 of Chapter 3, where it was assumed that this frequency is low enough to consider the measurements as static.

Fig. 5.2 shows the measurements of the spatial contrast sensitivity function at different temporal frequencies, while Fig. 5.3 shows the measurements of the temporal contrast sensitivity function at different spatial frequencies. The solid curves in both figures have been calculated with Eq. (5.2). The values used for $\tau_{1}$ and $\tau_{2}$ were $10.1 \mathrm{msec}$ and $11.8 \mathrm{msec}$, respectively, and the values used for $\sigma_{0}, \eta$, and $k$ were the same as used in section 3.9 .3 of Chapter 3 , being 0.53 arc $\mathrm{min}, 2.0 \%$ and 4.5 , respectively. The calculated curves were simultaneously fitted with the combined series of data of both figures. This could explain the worse fit of the model with the measurements at higher spatial frequencies in Fig 5.3. Apart from this, the model gives a good description of the trends shown by the measurements.

From Fig. 5.2, it can be seen that the spatial contrast sensitivity function has its normal bandpass shape at low temporal frequencies, whereas it gets a lowpass shape at high temporal frequencies. According to the here given model, this can simply be explained by the difference in the temporal behaviour of the photo-receptor signal and the subtracted lateral inhibition signal. At low temporal frequencies, 


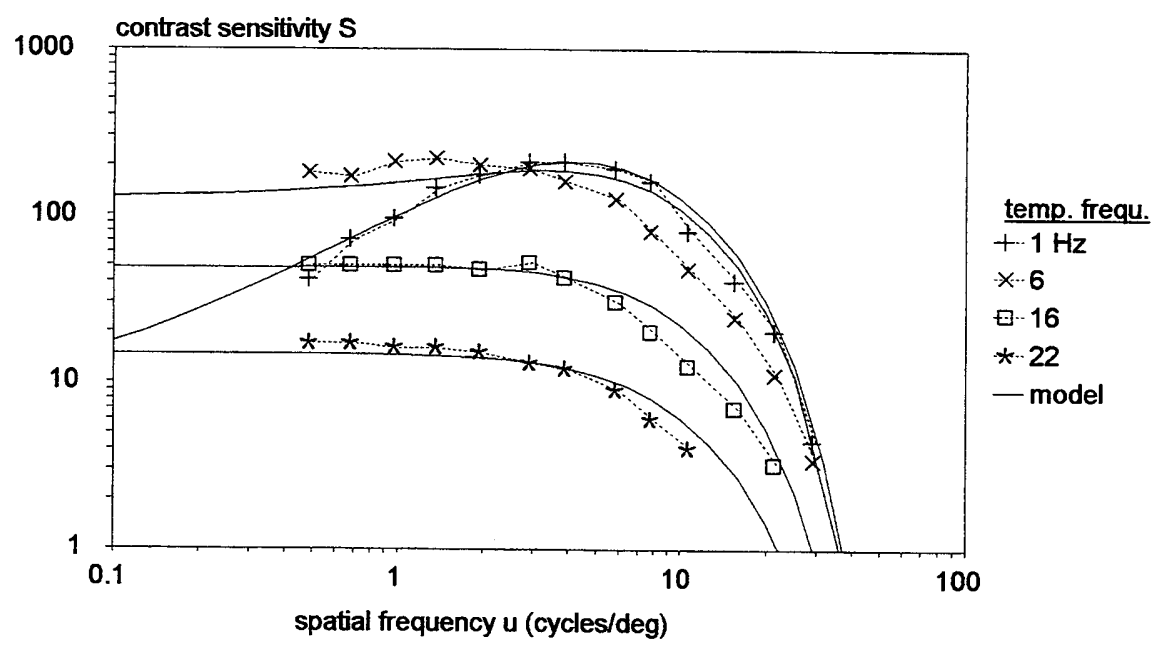

Figure 5.2: Spatial contrast sensitivity function measured by Robson (1966) at four different temporal frequencies. Luminance $20 \mathrm{~cd} / \mathrm{m}^{2}$. Field size $2.5^{\circ} \times 2.5^{\circ}$. Binocular viewing a natural pupil. The solid curves have been calculated with Eq. (5.2).

$H_{1}=H_{2} \approx 1$, so $H_{1}\left\{1-H_{2} F(u)\right\} \approx 1-F(u)$, and the contrast sensitivity function shows its normal spatial frequency behaviour. At medium temporal frequencies $\mathrm{H}_{2}$ is low, so $H_{1}\left\{1-H_{2} F(u)\right\} \approx H_{1}$. This means that the lateral inhibition has disappeared and that the contrast sensitivity curve is flat at low spatial frequencies. At higher temporal

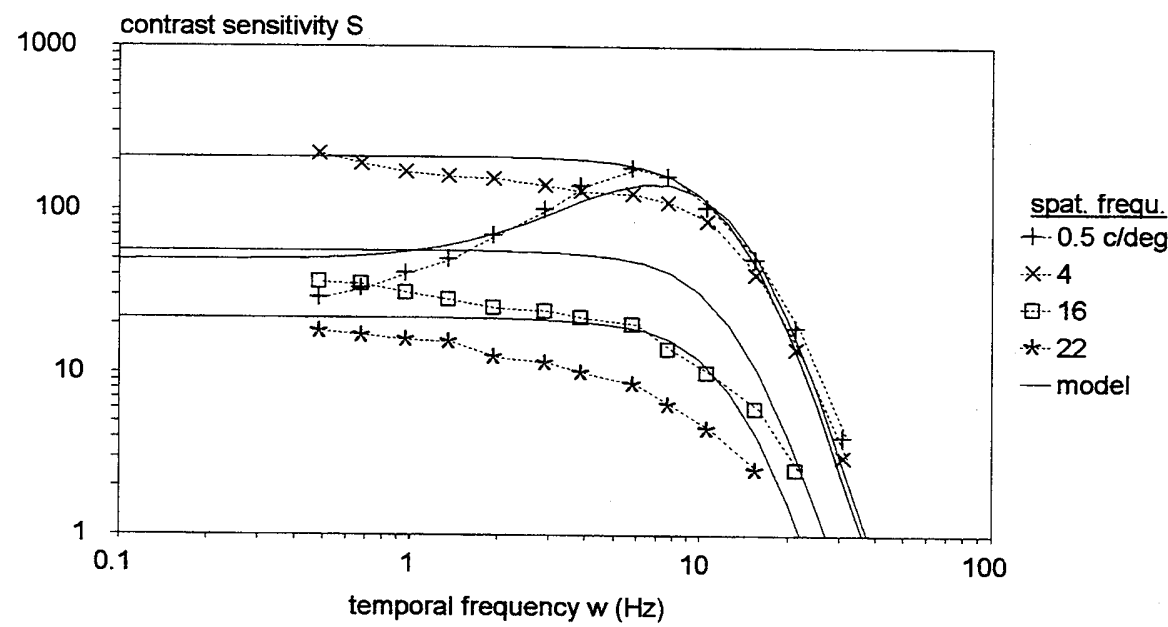

Figure 5.3: Temporal contrast sensitivity function measured by Robson (1966) at four different spatial frequencies. Luminance $20 \mathrm{~cd} / \mathrm{m}^{2}$. Field size $2.5^{\circ} \times 2.5^{\circ}$. Binocular viewing with a natural pupil. The solid curves have been calculated with Eq. (5.2). 
frequencies the shape remains the same but the level of the contrast sensitivity function further decreases proportionally with $H_{1}$.

Fig. 5.3 shows that the temporal contrast sensitivity function has a bandpass shape at low spatial frequencies and a lowpass shape at high spatial frequencies. According to the here given model, the bandpass character at low spatial frequencies is caused by a gradual decrease of the lateral inhibition when the temporal frequency increases. At low spatial frequencies $F(u) \approx 1$, so $H_{1}\left\{1-H_{2} F(u)\right\} \approx H_{1}\left(1-H_{2}\right)$. The factor 1- $\mathrm{H}_{2}$ causes a reduction of the contrast sensitivity at low temporal frequencies in the otherwise flat part of $H_{1}(w)$. For high spatial frequencies $F(u) \approx 0$, so $H_{1}\left\{1-H_{2} F(u)\right\}$ $\approx H_{1}$, which means that the contrast sensitivity curve is flat at low temporal frequencies. All curves start at low temporal frequencies from a value determined by the spatial contrast sensitivity function. Remarkable is the resemblance in shape between the curves of this figure and that of Fig. 5.2.

Kelly (1979) also made measurements of the spatial contrast sensitivity function at different temporal frequencies. They are shown in Fig. 5.4. He used a circular test pattern with a diameter of $7.5^{\circ}$ with a vertically oriented sinusoidal grating pattern generated on the screen of a CRT monitor. Viewing was monocular with an artificial pupil of $2.3 \mathrm{~mm}$ and with the use of special equipment to stabilize the position of the object on the retina. The retinal illuminance was $300 \mathrm{Td}$. The modulation threshold was determined by the method of adjustment. The measurements were made by one subject. The solid curves in the figure have been calculated

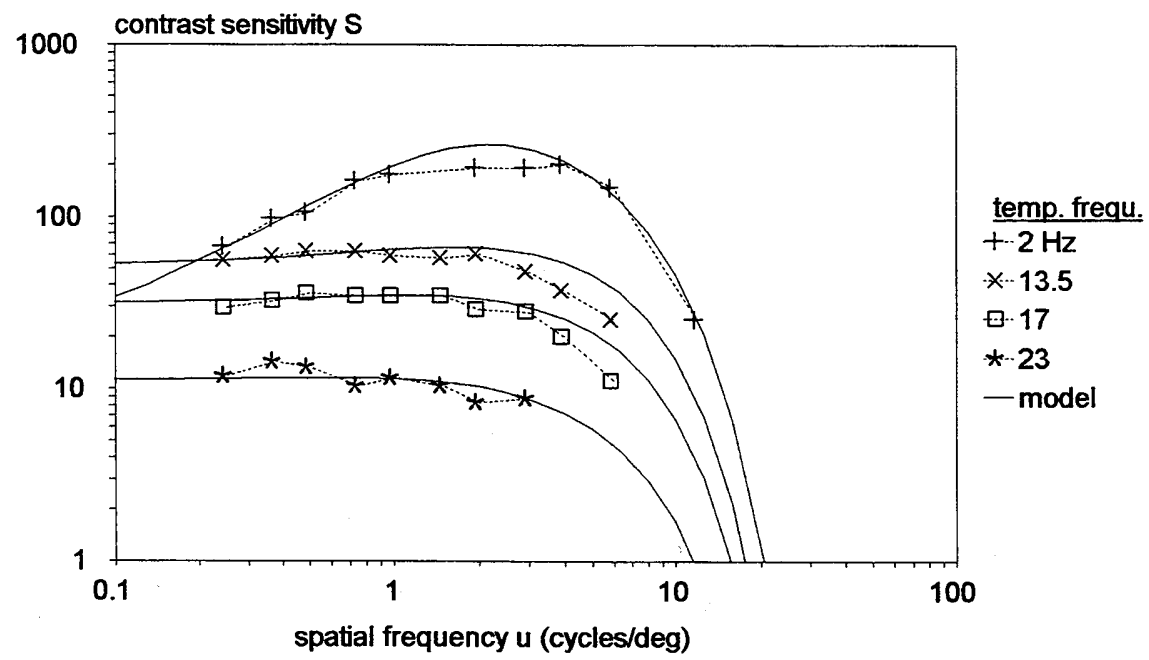

Figure 5.4: Spatial contrast sensitivity function measured by Kelly (1979) at four different temporal frequencies. Retinal illuminance $300 \mathrm{Td}$. Circular field with a diameter of $7.5^{\circ}$. Monocular viewing with an artificial pupil of $2.3 \mathrm{~mm}$. The solid curves have been calculated with Eq. (5.2). 
with Eq. (5.2). The values used for $\tau_{1}$ and $\tau_{2}$ were $11.1 \mathrm{msec}$ and $5.0 \mathrm{msec}$, respectively, and the values used for $\sigma_{0}, \eta$, and $k$ were 1.35 arc $\min , 1 \%$ and 3.2 , respectively. The curves have the same shape as the curves in Fig. 5.2. The calculated curves show a good agreement with the measurements.

\subsection{Temporal contrast sensitivity measurements}

Temporal contrast sensitivity measurements are usually not made with a spatial grating pattern, but with an evenly illuminated uniform field. From the general contrast sensitivity function given by Eq. (5.2), the spatial contrast sensitivity can be obtained by inserting $w=0$. However, the temporal contrast sensitivity function cannot be obtained by simply inserting $u=0$ in this equation. This insertion is correct for the factor $M_{\mathrm{lat}}(u)$, which becomes 1 , but is not correct regarding the function $F(u)$. The reason is that spatial field dimensions still play a role in temporal contrast sensitivity, because they determine the amount of lateral inhibition. For a uniform field the fundamental wave given by the size of the field is the strongest spatial frequency component of the object. The spatial frequency of this wave may therefore be considered to represent the spatial frequency content of the object in the function $F(u)$. If the field is square and has an angular width $X_{\mathrm{o}}$ in degrees, the spatial frequency of the fundamental wave in cycles/deg is

$$
u=\frac{1}{2 X_{0}}
$$

For a circular field, it may be assumed that the effective spatial frequency is equal to that of a square field with the same surface area. If the angular diameter of the field is $D$, the spatial frequency is then given by

$$
u=\frac{1}{\sqrt{\pi} D}
$$

The spatial frequency given by one of these equations has to be inserted in $F(u)$, instead of $u=0$, to obtain a correct expression for the temporal contrast sensitivity from Eq. (5.2).

That the spatial content of a uniform field can well be described by this spatial frequency is shown in Fig. 5.5, where two types of temporal contrast sensitivity measurements made by Kelly (1971) are given. One type was made with a circular uniform field with a diameter of $7^{\circ}$ and the other type was made with a grating pattern of the same size with a spatial frequency of 3 cycles/deg. The test objects were generated on the screen of a CRT monitor. They were observed with the right eye at a distance of $0.5 \mathrm{~m}$ through an artificial pupil of $2.3 \mathrm{~mm}$. The retinal illuminance was $1670 \mathrm{Td}$. The modulation threshold was determined by the method of adjustment. 


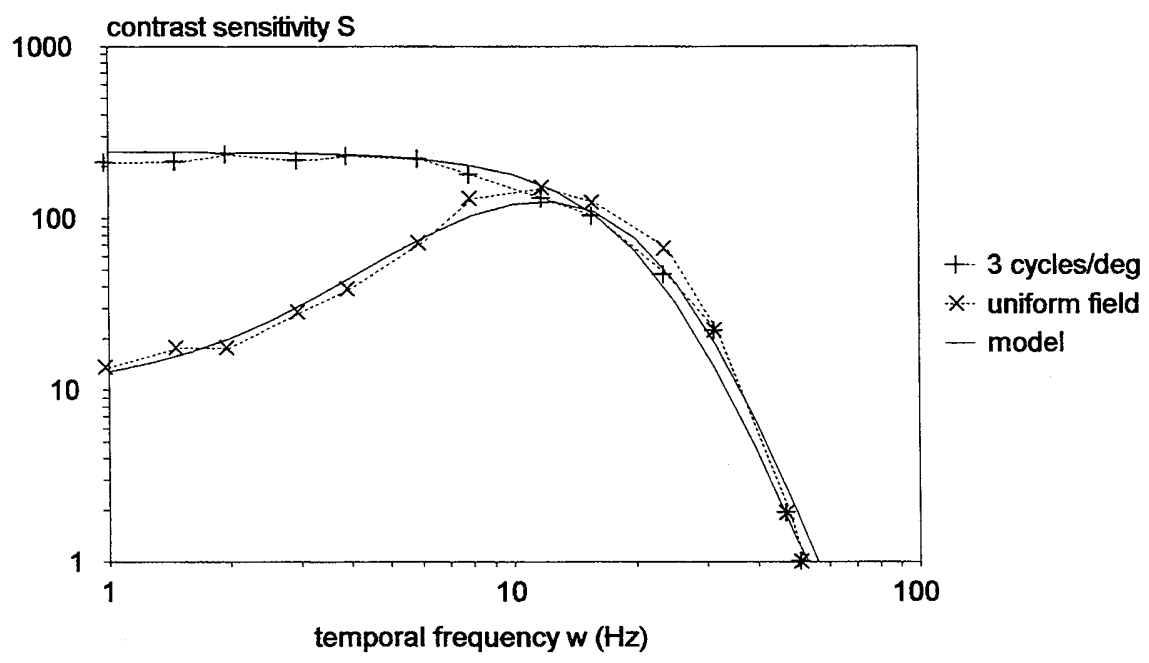

Figure 5.5: Temporal contrast sensitivity measured by Kelly (1971) for a grating with a spatial frequency of 3 cycles/deg and for a uniform field. Both fields are circular with a diameter of $7^{\circ}$. Retinal illuminance $1670 \mathrm{Td}$. Monocular viewing with an artificial pupil of $2.3 \mathrm{~mm}$. The solid curves have been calculated with Eq. (5.2) using for the uniform field the value of $u$ given by Eq. (5.10).

The subject was a female (LH), 20 years of age. The solid curves in the figure have been calculated with Eq. (5.2) where for the uniform field, the fundamental spatial frequency given by Eq. (5.10) was used for $F(u)$ and $M_{\text {spat }}$ was set to 1 . The values used for $\tau_{1}$ and $\tau_{2}$ were $6.9 \mathrm{msec}$ and $6.1 \mathrm{msec}$, respectively, and the values used for $\sigma_{0}, \eta$, and $k$ were 0.5 arc min, $3 \%$ and 5.6 , respectively. The simultaneous fit of both curves with the measurement data shows, that the temporal contrast sensitivity of a uniform field can well be described by Eq. (5.2) by using the spatial frequency of the fundamental wave in $F(u)$.

Another example of temporal contrast sensitivity measurements for a uniform field is given in Fig. 5.6. This figure shows measurements by Roufs \& Blommaert (1981) for a circular field with a diameter of $1^{\circ}$ and a retinal illumination of $1200 \mathrm{Td}$. Viewing was monocular with an artificial pupil of $2 \mathrm{~mm}$. The modulation threshold was determined by measuring the psychometric function and using the modulation at $50 \%$ detection probability. Subject was the first author (JAJR), 46 years of age. The solid curve was calculated in the same way as for the uniform field in Fig. 5.5. The values used for $\tau_{1}$ and $\tau_{2}$ were $7.2 \mathrm{msec}$ and $13.6 \mathrm{msec}$, respectively, and the values used for $\eta$ and $k$ were $3 \%$ and 3.9 , respectively. The curve is very similar to the curve for the uniform field measured by Kelly, which was shown in Fig. 5.5. The fit between calculations and measurements is very good.

Besides the fundamental spatial frequency, in the uniform field also higher 


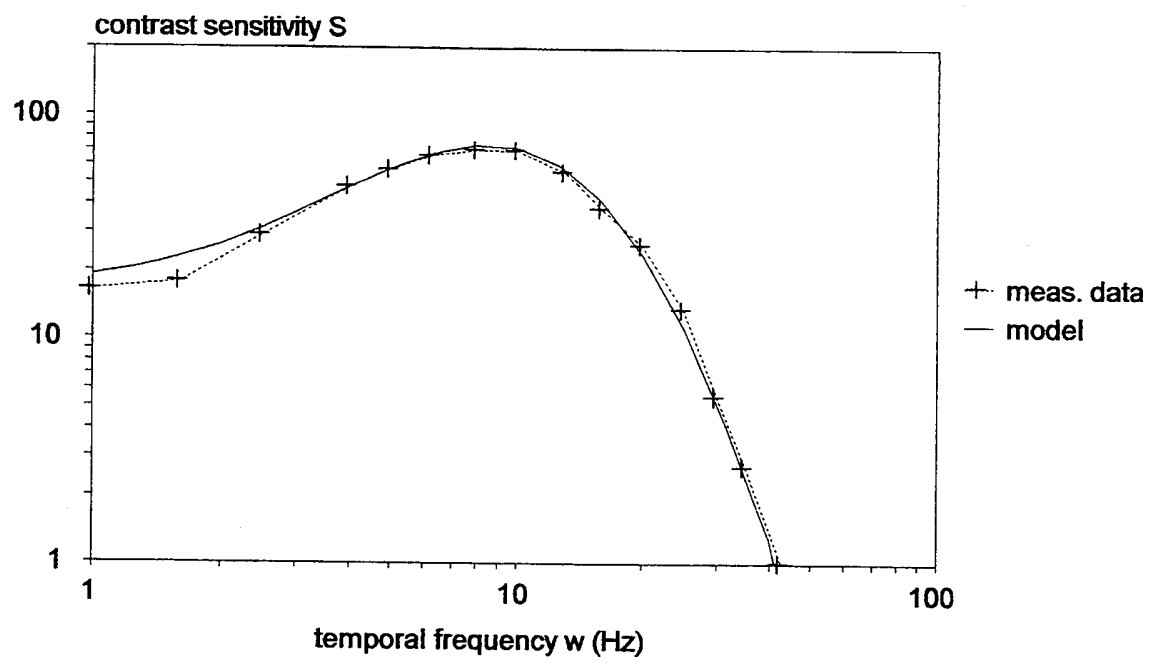

Figure 5.6: Temporal contrast sensitivity measured by Roufs and Blommaert (1981) for a circular uniform field with a diameter of $1^{\circ}$ and a retinal illuminance of $1200 \mathrm{Td}$. Monocular viewing with an artificial pupil of $2 \mathrm{~mm}$. The solid curve has been calculated with Eq. (5.2) using the value of $u$ given by Eq. (5.10).

harmonics can play a role in the detection process. In the spatial frequency spectrum of a uniform field only odd harmonics are present. For small fields, the spatial frequency of the harmonic falls in the declining part of the spatial contrast sensitivity function and can therefore be neglected. For large fields the fundamental spatial frequency is low and the spatial frequency of the higher harmonics falls partly in the rising part of the spatial contrast sensitivity function. This is illustrated by contrast sensitivity measurements made by Campbell \& Robson (1968) for sine-wave and square wave gratings (See, for instance, their Fig. 3). Therefore, with large fields generally a better fit with the measurements is obtained by using for the spatial frequency in $F(u)$ the third harmonic, instead of the fundamental spatial frequency.

The spatial frequency used in $F(u)$ has influence on contrast sensitivity only at low temporal frequencies. At temporal frequencies above $10 \mathrm{~Hz}, F(u)$ has practically no influence because $\mathrm{H}_{2}(w)$ becomes too small. This can be seen from Fig. 5.5 where the curves above $10 \mathrm{~Hz}$ nearly coincide.

\subsection{Effect of a surrounding field}

Most temporal contrast sensitivity measurements are made with a dark area surrounding the test field. However, sometimes a stationary surrounding field is used with a constant luminance equal to the average luminance of the test field. This method was, 


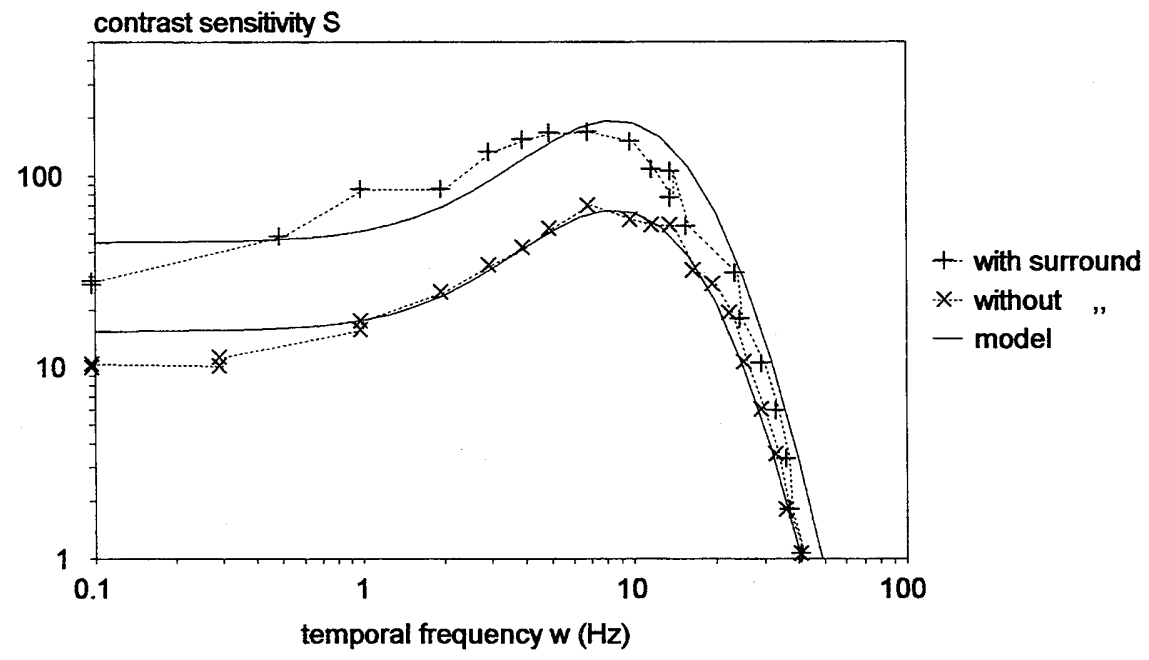

Figure 5.7: Temporal contrast sensitivity measured by Roufs (1972a) for a circular uniform field with a diameter of $1^{\circ}$ with and without a stationary equiluminous surround. Retinal illuminance $1150 \mathrm{Td}$. Monocular viewing with an artificial pupil of $2 \mathrm{~mm}$. The solid curves have been calculated with Eq. (5.2), using for $u$ the value given by Eq. (5.10) and using for the measurements with surrounding field an effective field size with a threefold diameter.

for instance, used by de Lange in his measurements. Roufs (1972a) pointed out that this detail may not be neglected. With a surrounding field the temporal contrast sensitivity is increased. The effect of the surround can be taken into account in the calculations by assuming that the effective size of the object is increased, whereas the fundamental spatial frequency to be used in $F(u)$ remains the same.

To investigate the effect of a surrounding field, Roufs (1972a) measured the temporal contrast sensitivity of a uniform field with and without a surrounding field. The test field was a circular field with a diameter of $1^{\circ}$ and a retinal illuminance of $1150 \mathrm{Td}$. Viewing was monocular with an artificial pupil of $2 \mathrm{~mm}$. The threshold was determined by measuring the psychometric function and using the modulation at $50 \%$ detection probability. The author, JAJR, was the observer, 39 years of age at the time of this investigation.

Measurements and calculations are shown in Fig. 5.7. Both solid curves have been calculated with Eq. (5.2) using for $u$ the value given by Eq. (5.10). The values used for $\tau_{1}$ and $\tau_{2}$ were $7.3 \mathrm{msec}$ and $13.2 \mathrm{msec}$, respectively, and the values used for $\eta$ and $k$ were $3 \%$ and 4.2 , respectively. For the curve with a surrounding field, an integration area with a threefold diameter had to be assumed to obtain an agreement with the measurements. This size increase corresponds with the addition of an annular ring with a width equal to the diameter of the stimulus. From the figure, it 
can be seen that in this way a good description of the measurements is obtained. This rule will therefore also be applied here in other situations where the uniform field is surrounded by an equiluminous stationary field. At the very low frequencies used in this experiment, one can observe some deviation between measurements and calculations. However, one should realize that the measurement of contrast sensitivity at low temporal frequencies is very difficult. At a temporal frequency of $0.1 \mathrm{~Hz}$, one cycle lasts $10 \mathrm{sec}$. It is well known from experiments that it is very difficult for the eye to observe luminance chances that happen so slowly.

\subsection{Effect of retinal illuminance and field size on the time constants}

Temporal contrast sensitivity measurements made at different retinal illuminance cannot be described with a single value for the time constants $\tau_{1}$ and $\tau_{2}$. The same holds for measurements made at different field sizes. The time constants appear to depend from both quantities. They decrease with increasing retinal illuminance and with increasing field size. The dependence on field size is probably related with the variation of cone density and ganglion cell density over the retina. By analyzing the published temporal contrast sensitivity measurements given in this section, it was found that the dependence on retinal illuminance and field size can approximately be described by the following equations:

and

$$
\tau_{1}=\frac{\tau_{10}}{1+0.55 \ln \left\{1+\left(1+\frac{D}{1}\right)^{0.6} \frac{E}{3.5}\right\}}
$$

$$
\tau_{2}=\frac{\tau_{20}}{1+0.37 \ln \left\{1+\left(1+\frac{D}{3.2}\right)^{5} \frac{E}{120}\right\}}
$$

where $\tau_{10}$ and $\tau_{20}$ are fixed time constants that do not depend on retinal illuminance and field size, $D$ is the field diameter in degrees, and $E$ is the retinal illuminance in Troland.

Figs. 5.8 and 5.9 show how $\tau_{1}$ and $\tau_{2}$, respectively, vary with retinal illuminance according to these formulas. Curves are given for two field diameters: $1^{\circ}$ and $59^{\circ}$ with measured data points derived from temporal contrast sensitivity measurements. The data for $1^{\circ}$ were derived from measurements by Roufs (1972a) and the data for $59^{\circ}$ were derived from measurements by Kelly (1961). These measurements will be treated in more detail in the following part of this section. From the figures, it can be 


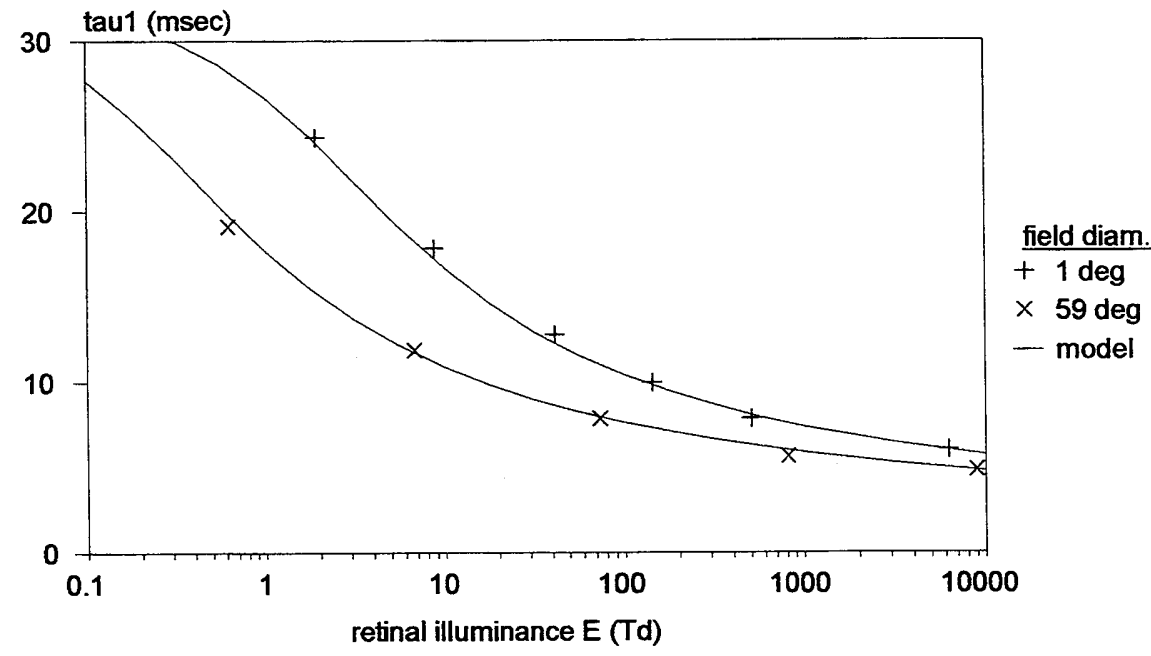

Figure 5.8: Variation of the time constant $\tau_{1}$ with retinal illuminance, calculated with Eq. (5.11) for a field diameter of $1^{\circ}$ and $59^{\circ}$. The data points for $1^{\circ}$ are derived from measurements by Roufs (1972a) and the data points for $59^{\circ}$ are derived from measurements by Kelly (1961).

seen that the field size mainly an influence on $\tau_{2}$. The dependence of $\tau_{2}$ on field size is further shown in Fig. 5.10 for a fixed retinal illuminance of $62 \mathrm{Td}$. The data points in this figure were derived from measurements by Roufs \& Bouma (1980). These

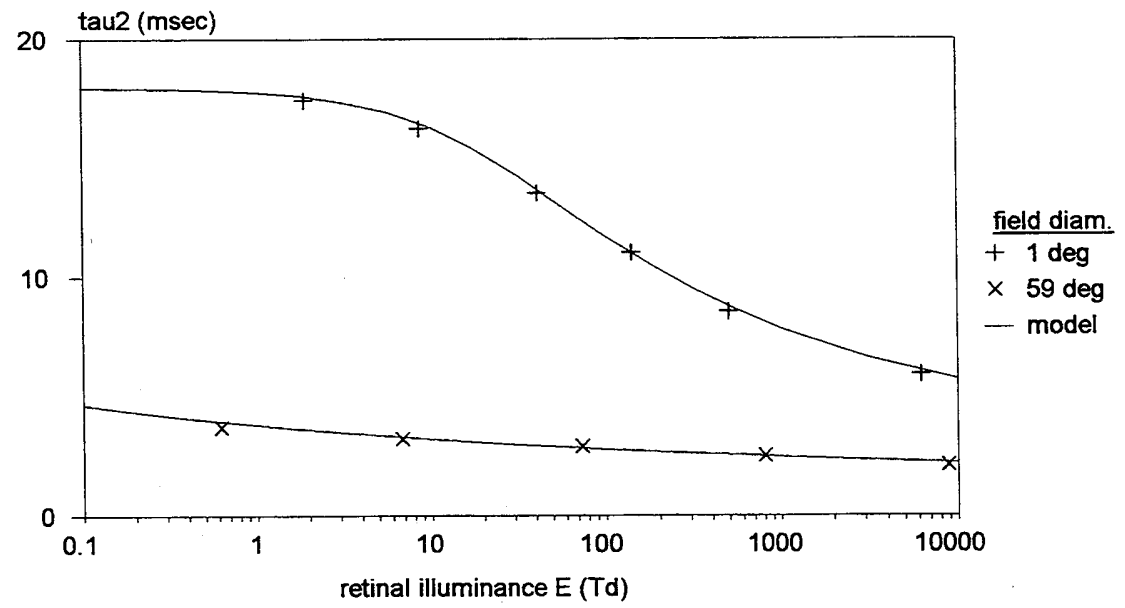

Figure 5.9: Variation of the time constant $\tau_{2}$ with retinal illuminance, calculated with Eq. (5.12) for a field diameter of $1^{\circ}$ and $59^{\circ}$. The data points for $1^{\circ}$ are derived from measurements by Roufs (1972a) and the data points for $59^{\circ}$ are derived from measurements by Kelly (1961). 


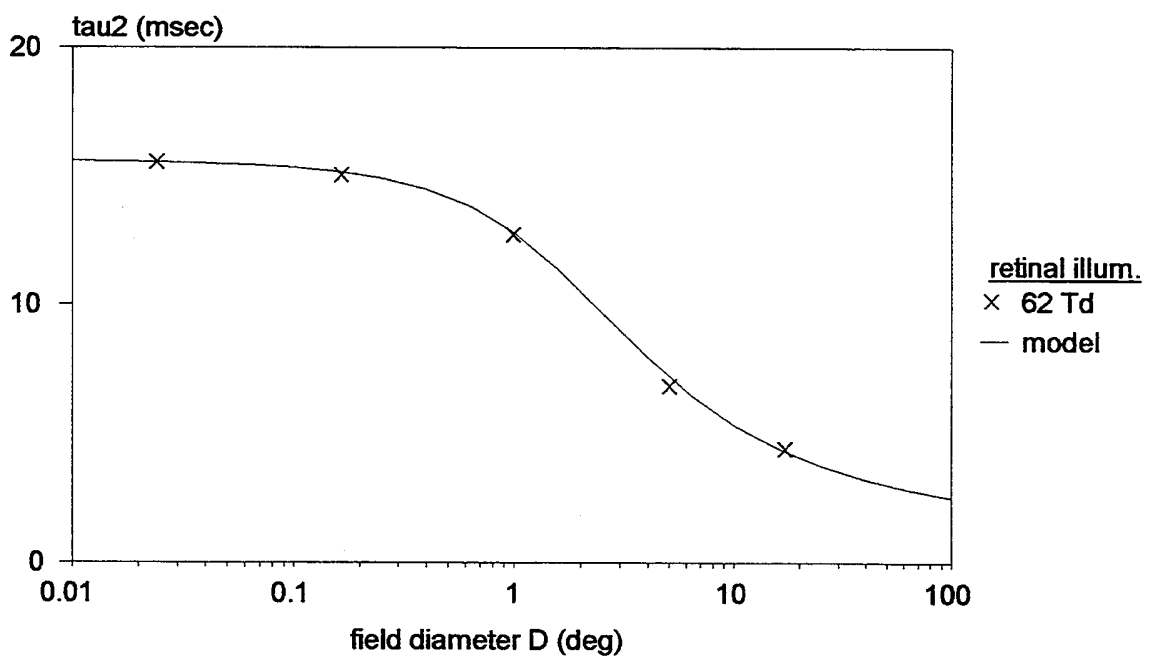

Figure 5.10: Variation of the time constant $\tau_{2}$ with field diameter, calculated with Eq. (5.12) for a retinal illuminance of $62 \mathrm{Td}$. The data points are derived from measurements by Roufs \& Bouma (1980).

measurements will also be treated in more detail in the following part of this section.

At very low retinal illuminance and very small field size the time constants $\tau_{1}$ and $\tau_{2}$ become equal to $\tau_{10}$ and $\tau_{20}$, respectively. The actual values of $\tau_{1}$ and $\tau_{2}$ are characterized by these constants. Ideally $\tau_{10}$ and $\tau_{20}$ have the same value for all measurements, but in practice, they differ for different subjects and for different experiments. In Figs. 5.8 to $5.10, \tau_{10}=32 \mathrm{msec}$ and $\tau_{20}=18 \mathrm{msec}$. These values represent an average value for the given measurements. Eqs. (5.11) and (5.12) will be used in the following temporal contrast sensitivity measurements for a simultaneous description of the curves for different field size or illuminance. For these measurements, the values of $\tau_{10}$ and $\tau_{20}$ will be determined that give a best fit with the data. The results will be given in Table 1 at the end of this section.

Fig. 5.11 shows so obtained calculated curves with the measurement data for temporal contrast sensitivity measurements made by Roufs \& Bouma (1980) for a large range of field sizes. The data given in Fig. 5.10 were obtained from this investigation. The measurements were made with a retinal illuminance of $62 \mathrm{Td}$ and with circular fields of which the diameter was varied from $1.5 \operatorname{arc} \min \left(0.025^{\circ}\right)$ to 1060 arc min $\left(17.7^{\circ}\right)$. Viewing was monocular with the left eye through an artificial pupil of $2 \mathrm{~mm}$. Subject was HJM, 25 years of age. The solid curves have been calculated with Eqs. (5.2) and Eqs. (5.10) through (5.12) with a simultaneous fit for all curves. The values of $\tau_{2}$ shown in Fig. 5.10 were obtained from the same data by a non-simultaneous fit. For the two largest field sizes in the figure the third harmonic 


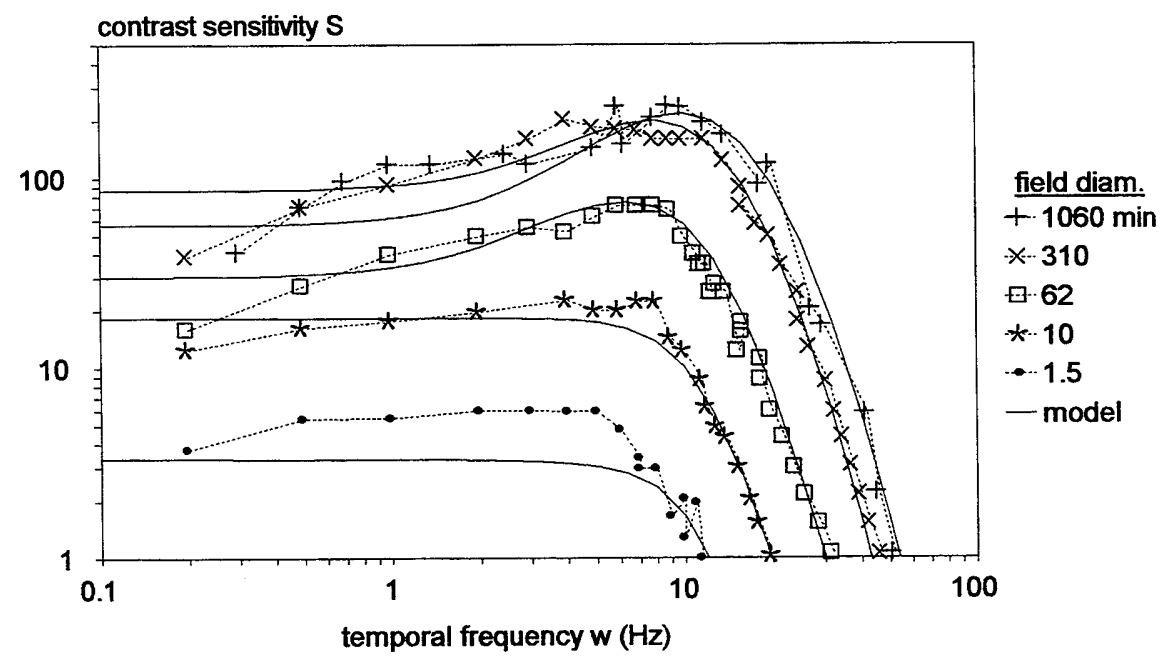

Figure 5.11: Temporal contrast sensitivity measured by Roufs \& Bouma (1980) for circular fields with different field diameters. Retinal illuminance $62 \mathrm{Td}$. Monocular viewing with an artificial pupil of $2 \mathrm{~mm}$. The solid curves have been calculated with Eq. (5.2) and Eqs. (5.10) through (5.12).

of the spatial frequency was used in $F(u)$, instead of the fundamental frequency, for the reasons mentioned in section 5.5. The values of $\tau_{10}$ and $\tau_{20}$ were $32 \mathrm{msec}$ and 18 msec, respectively, and the values used for $\eta$ and $k$ were $6 \%$ and 2.1 , respectively. Apart from a deviation for the very small field diameter of 1.5 arc min $\left(0.025^{\circ}\right)$, measurements and calculations show a very good agreement over a large range of field sizes. This means that the dependence of the time constants on field size is well described by Eqs. (5.11) and (5.12).

Fig. 5.12 shows measurements by de Lange (1958a) for a large range of retinal illuminance levels extending from $0.375 \mathrm{Td}$ to $1000 \mathrm{Td}$. He used a circular test field with a diameter of $2^{\circ}$ surrounded by a uniform field with a diameter of $60^{\circ}$ at the same luminance. Viewing was monocular with an artificial pupil of $2.8 \mathrm{~mm}$. The modulation threshold was determined by the method of adjustment. Two subjects took part in the experiments. In Fig. 5.10 the data are given for one subject, subject $\mathrm{L}$, the author, 52 years of age. The solid curves in the figure have been calculated with Eq. (5.2) and Eqs. (5.10) through (5.12). The presence of the surrounding field was taken into account in the calculations by increasing the diameter of the integration area with a factor 3 , as mentioned in section 5.6. The values of $\tau_{10}$ and $\tau_{20}$ were $29 \mathrm{msec}$ and $18 \mathrm{msec}$, respectively, and the values used for $\eta$ and $k$ were $2 \%$ and 2.8 , respectively. Apart from some considerable deviations, the general trends of the measurements are well described by the calculated curves. The deviations can partly be explained by the primitive conditions under which these first temporal contrast sensitivity measurements had to be made. The deviations of the two lowest curves can 


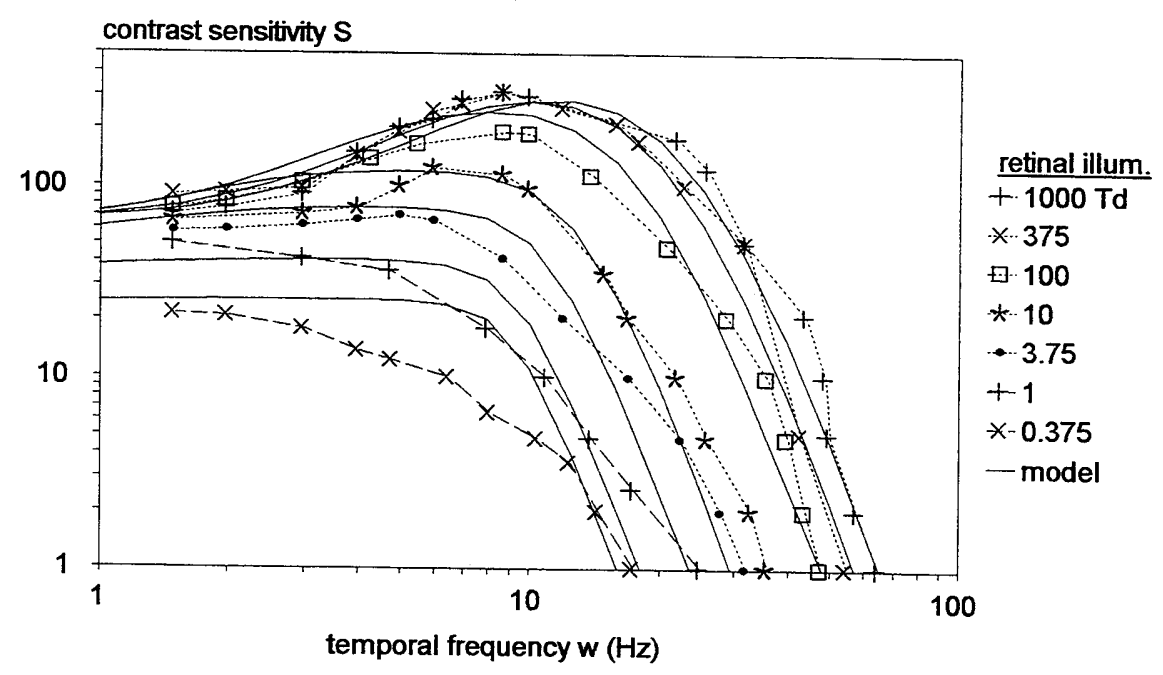

Figure 5.12: Temporal contrast sensitivity curves measured by de Lange (1958a) for a circular field with a diameter of $2^{\circ}$ and a large range of retinal illuminance levels. Monocular viewing with an artificial pupil of $2.8 \mathrm{~mm}$. The solid curves have been calculated with Eq. (5.2) and Eqs. (5.10) through (5.12).

be explained by the fact that the retinal illuminance for these curves is scotopic, instead of photopic.

After the investigation by de Lange, other investigators (e.g., Kelly and Roufs) studied intensively the dependence of temporal contrast sensitivity on luminance. Because of the pioneering work by de Lange, temporal contrast sensitivity curves are often called de Lange curves.

Fig. 5.13 shows measurements by Kelly (1961) for a large circular test field with a smooth edge and a range of retinal illuminance levels extending from $0.65 \mathrm{Td}$ to $9300 \mathrm{Td}$. The $50 \%$ diameter of the test field was $59^{\circ}$. The data for $59^{\circ}$ given in Figs. 5.8 and 5.9 were obtained from this investigation. Viewing was monocular with an artificial pupil of $1.55 \mathrm{~mm}$. The modulation threshold was determined by the method of adjustment. The author, DHK, was the observer. The solid curves in the figure have been calculated with Eq. (5.2) and Eqs. (5.10) through (5.12) with a simultaneous fit for all curves. The values of $\tau_{1}$ and $\tau_{2}$ shown in Figs. 5.8 and 5.9, respectively, were obtained from a non-simultaneous fit. Because of the large field, the third harmonic of the fundamental spatial frequency was used for the spatial frequency in $F(u)$. See section 5.5. The values of $\tau_{10}$ and $\tau_{20}$ were $31 \mathrm{msec}$ and 17 msec, respectively, and the values used for $\eta$ and $k$ were $3 \%$ and 3.6. Apart from the curve for $0.06 \mathrm{Td}$, where vision is scotopic, the agreement between measurements and calculations is very good. 


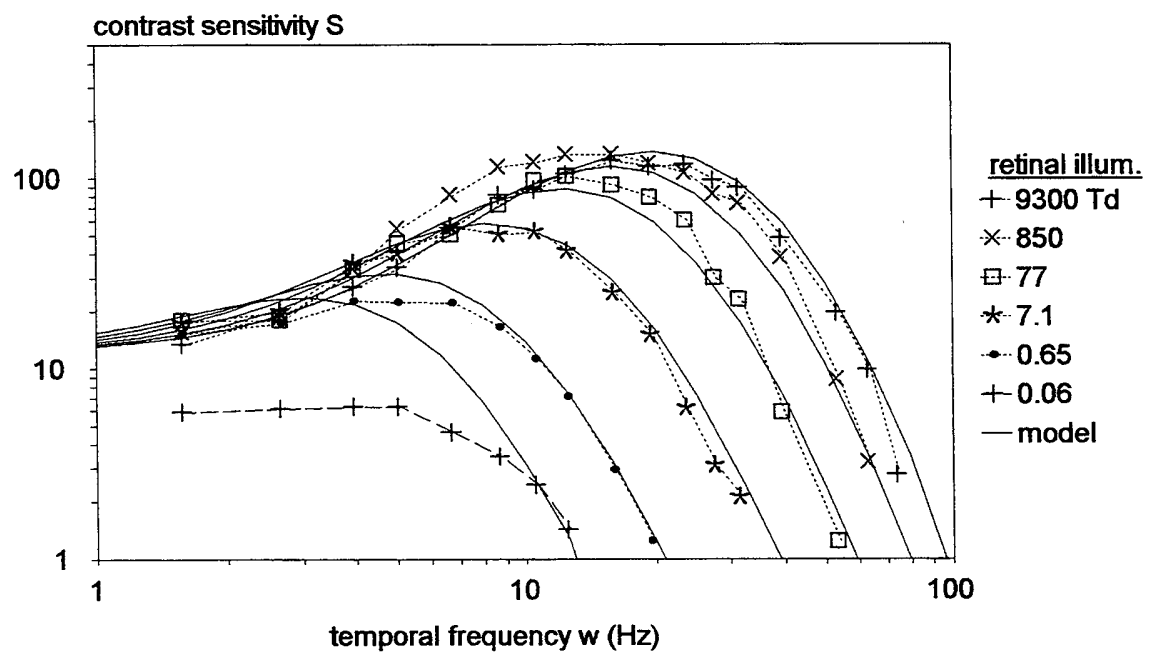

Figure 5.13: Temporal contrast sensitivity measured by Kelly (1961) for a circular field with a diameter of $59^{\circ}$ and a large range of retinal illuminance levels. Monocular viewing with an artificial pupil of $1.55 \mathrm{~mm}$. The solid curves have been calculated with Eq. (5.2) and Eqs. (5.10) through (5.12) using the third harmonic of the fundamental spatial frequency.

Fig. 5.14 shows similar measurements by Roufs (1972a) for a circular test field with a diameter of $1^{\circ}$ and a range of retinal illuminance levels extending from $2 \mathrm{Td}$ to

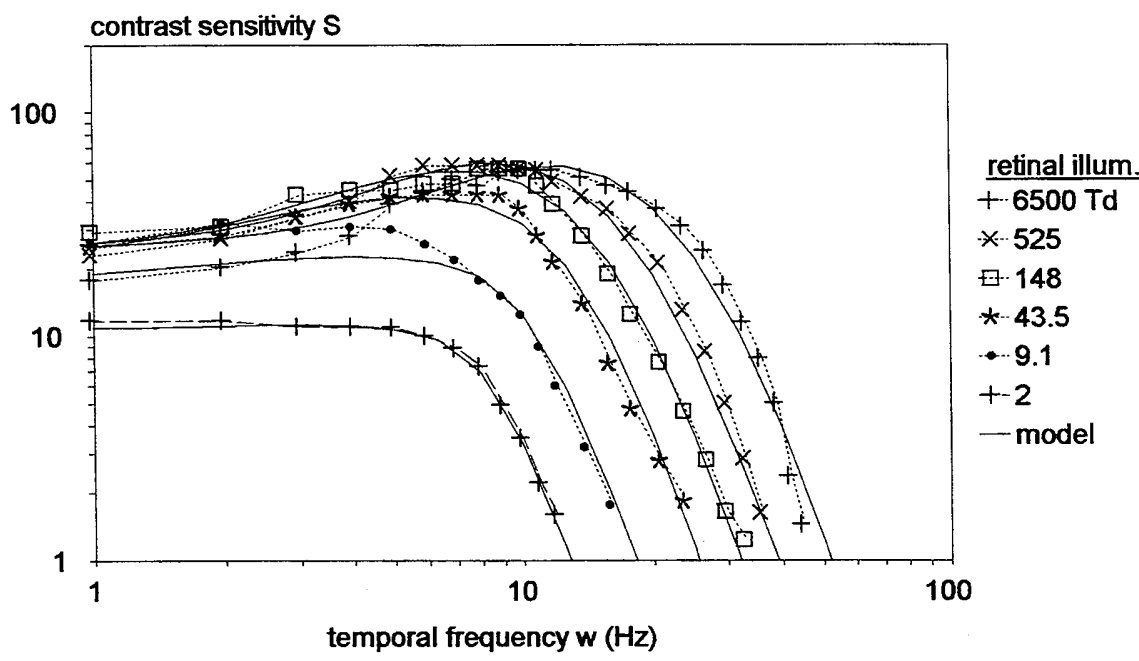

Figure 5.14: Temporal contrast sensitivity curves measured by Roufs (1972a) for a circular field with a diameter of $1^{\circ}$ and a large range of retinal illuminance levels. Monocular viewing with an artificial pupil of $2 \mathrm{~mm}$. The solid curves have been calculated with Eq. (5.2) and Eqs. (5.10) through (5.12). 


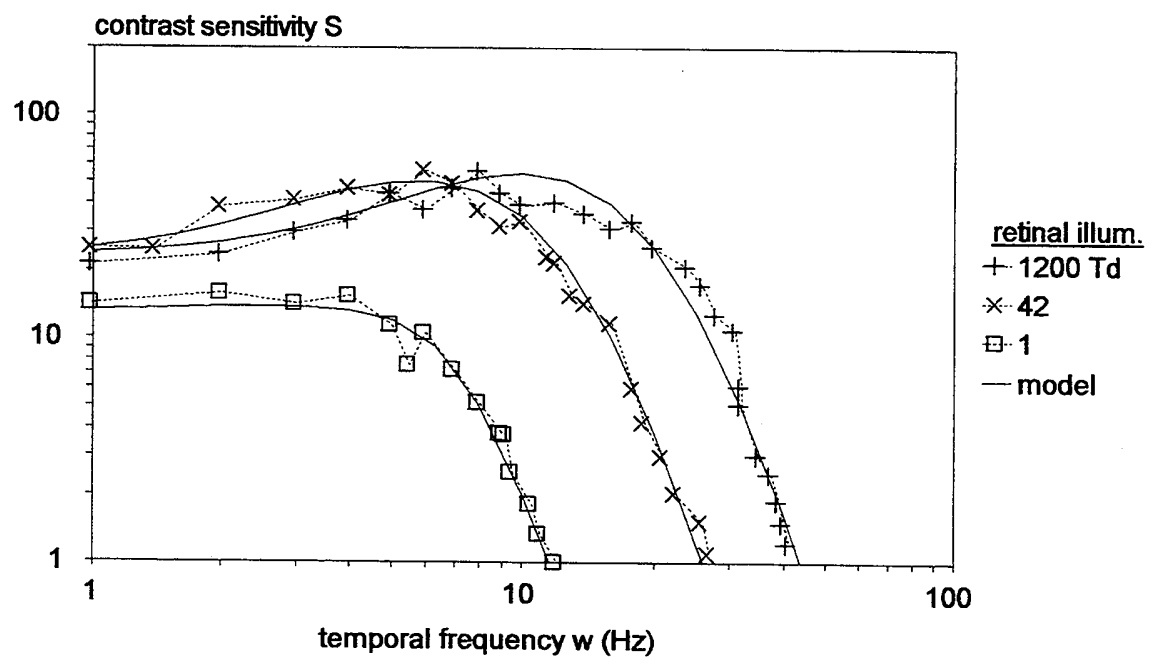

Figure 5.15: Temporal contrast sensitivity curves measured by Roufs (1973) for three different retinal illuminance levels. Apart from a different observer, further conditions are the same as in Fig. 5.14.

$6500 \mathrm{Td}$. The data points for $1^{\circ}$ given in Figs. 5.8 and 5.9 were obtained from these measurements. Viewing was monocular with an artificial pupil of $2 \mathrm{~mm}$. The modulation threshold was determined by measuring the psychometric function and using the modulation at $50 \%$ detection probability. Subject was RK, 18 years of age. The solid curves in the figure have been calculated with Eq. (5.2) and Eqs. (5.10) through (5.12) with a simultaneous fit for all curves. The values of $\tau_{1}$ and $\tau_{2}$ shown in Figs. 5.8 and 5.9, respectively, were obtained from a non-simultaneous fit. The values of $\tau_{10}$ and $\tau_{20}$ were $33 \mathrm{msec}$ and $18 \mathrm{msec}$, respectively, and the values used for $\eta$ and $k$ were $2 \%$ and 2.6 , respectively. From the figure, it can be seen that the general agreement between measurements and calculations is very good.

Fig. 5.15 shows other measurements by Roufs (1973) for three retinal illuminance levels: $1 \mathrm{Td}, 42 \mathrm{Td}$, and $1200 \mathrm{Td}$. The measurements were made under the same conditions as for the measurements described above, but with a different subject, JAJR, the author, 39 years of age at the time of this investigation. The solid curves in the figure have been calculated with Eq. (5.2) and Eqs. (5.10) through (5.12). The values of $\tau_{10}$ and $\tau_{20}$ were $32 \mathrm{msec}$ and $17 \mathrm{msec}$, respectively, and the values used for $\eta$ and $k$ were $8 \%$ and 2.8 , respectively. The agreement between measurements and calculations is very good.

Fig. 5.16 shows measurements by Swanson et al. (1987) for a circular test field with a diameter of $2^{\circ}$ and for two retinal illuminance levels: $9 \mathrm{Td}$ and $900 \mathrm{Td}$. Viewing was monocular with an artificial pupil of $2 \mathrm{~mm}$. The modulation threshold 


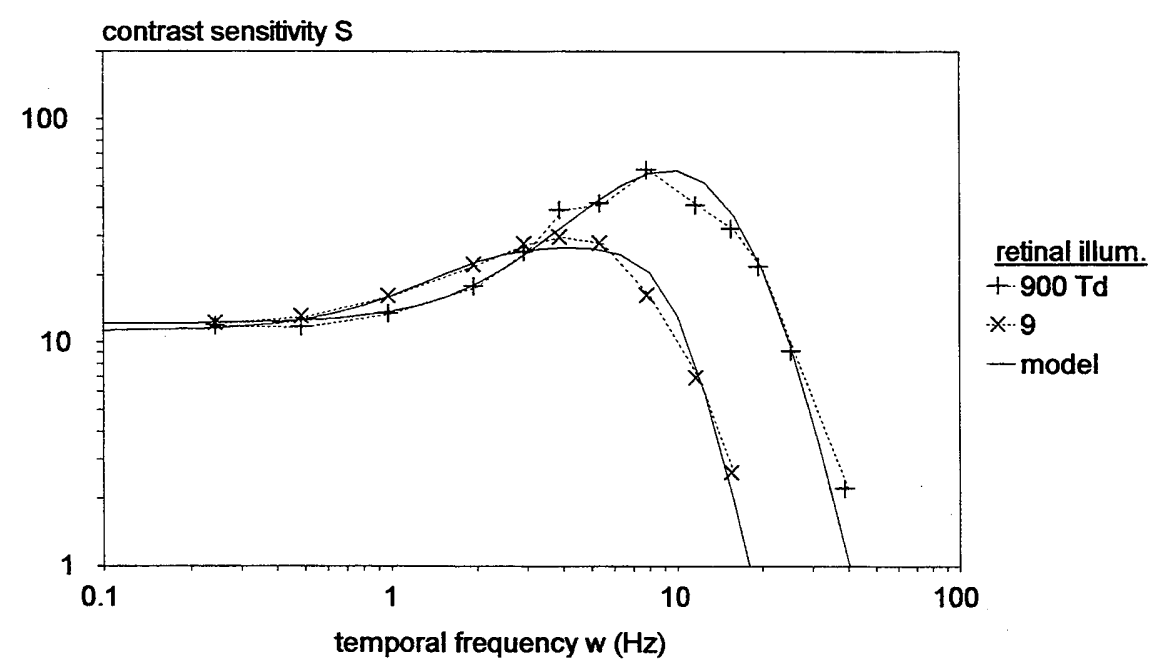

Figure 5.16: Temporal contrast sensitivity curves measured by Swanson et al. (1987) for a circular field with a diameter of $2^{\circ}$ and two different retinal illuminance levels. Monocular viewing with an artificial pupil of $2 \mathrm{~mm}$. The solid curves have been calculated with Eq. (5.2) and Eqs. (5.10) through (5.12).

was determined by the method of adjustment. In the figure, the results for two of the four measured illuminance levels were used. Two subjects took part in the experiments: WS and TU, the first two authors. The given data are the average for these subjects. The solid curves have been calculated with Eq. (5.2) and Eqs. (5.10) through (5.12). The values of $\tau_{10}$ and $\tau_{20}$ were $35 \mathrm{msec}$ and $23 \mathrm{msec}$, respectively, and the values used for $\eta$ and $k$ were $3 \%$ and 5.3 , respectively. The calculated curves show a good agreement with the measurements.

From the evaluation of the measurement data given in this section, it appears that Eqs. (5.11) and (5.12) give a good description of the dependence of the time constants on retinal illuminance and field size. In Table 5.1 a survey is given of the values of $\tau_{10}$ and $\tau_{20}$ used for the evaluation of these measurements. Besides these values, also values are added that were derived from the measurements given in the preceding sections. From the table, it can be seen that $\tau_{10}$ shows a concentration around $32 \mathrm{msec}$ and $\tau_{20}$ shows a concentration around $18 \mathrm{msec}$. These values may be considered as typical values for these constants. Contrary to what one should expect, the data in the table do not show a clear effect of age. However, this corresponds with the results of an investigation by Tyler (1989) who measured the temporal contrast sensitivity as a function of age with a large number of subjects. He found only a very small age dependence for subjects between 16 and 70 years of age. 
Table 5.1: Time constants $\tau_{10}$ and $\tau_{20}$ used for the measurements

\begin{tabular}{|l|c|c|c|c|c|c|}
\hline \multicolumn{1}{|c|}{ author } & $\begin{array}{c}\text { field } \\
\text { size } \\
(\mathrm{deg})\end{array}$ & $\begin{array}{c}\text { retinal } \\
\text { illum. } \\
(\mathrm{Td})\end{array}$ & $\begin{array}{c}\tau_{10} \\
(\mathrm{msec})\end{array}$ & $\begin{array}{c}\tau_{20} \\
(\mathrm{msec})\end{array}$ & subject & $\begin{array}{c}\text { age } \\
\text { (years) }\end{array}$ \\
\hline Robson (1966) & 2.5 & 320 & 41 & 31 & JGR & \pm 26 \\
\hline Kelly (1979) & 7.5 & 300 & 46 & 18 & - & - \\
\hline Kelly (1971) & 7 & 1670 & 35 & 25 & LH & 20 \\
\hline Roufs et al. (1981) & 1 & 1200 & 32 & 32 & JAJR & 46 \\
\hline Roufs (1972a) & 1 & 1150 & 32 & 31 & JAJR & 39 \\
\hline Roufs et al. (1980) & varied & 62 & 32 & 18 & HJM & 25 \\
\hline de Lange (1958) & 2 & varied & 29 & 18 & L & 52 \\
\hline Kelly (1961) & 59 & varied & 31 & 17 & DHK & \pm 25 \\
\hline Roufs (1972a) & 1 & varied & 33 & 18 & RK & 18 \\
\hline Roufs (1973) & 1 & varied & 32 & 17 & JAJR & 39 \\
\hline Swanson et al. (1987) & 1 & varied & 35 & 23 & av. 2 & - \\
\hline
\end{tabular}

\subsection{Flicker sensitivity: Ferry-Porter law}

The study of temporal contrast sensitivity was stimulated by the introduction of motion picture films at the end of the nineteenth century and was stimulated again by the introduction of television halfway the twentieth century. The reason for this interest was the annoying flicker effects that can occur by the simulation of movements with a repeated sequence of images. At the end of the nineteenth century and the beginning of the twentieth century Ferry (1892) and Porter (1902) found that the frequency above which flicker becomes invisible, increases linearly with the logarithm of the luminance. This law is known as Ferry-Porter law. The frequency above which the flicker is not visible anymore is called the critical flicker frequency or CFF. At that time, measurements had to be made with very primitive means. For a part of his experiments, Porter used candles as light source. Later, when television started, the higher luminance of television images caused a new interest for the flicker problem. In this time de Lange did his well-known investigation on the fundamental aspects of periodic temporal luminance variations. 


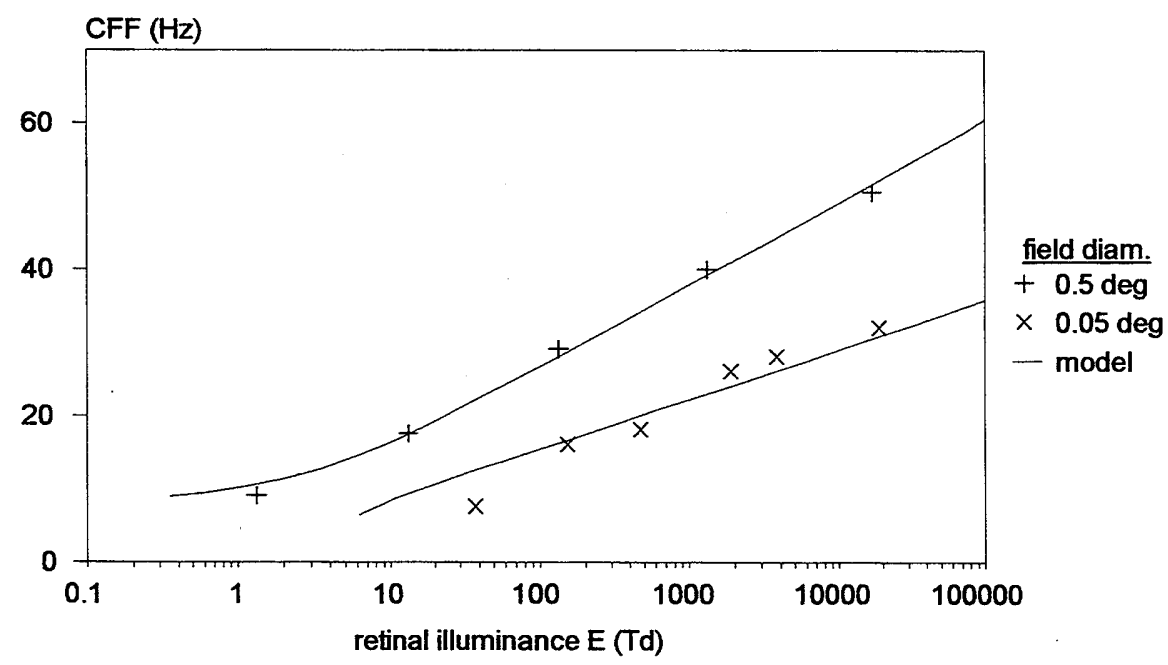

Figure 5.17: Critical flicker frequency as a function of retinal illuminance measured by Tyler \& Hamer (1990) for a circular field with a diameter of $0.5^{\circ}$ and $0.05^{\circ}$ with a $100 \%$ modulated sinusoidal temporal luminance variation. Viewing was monocular. The solid curves have been calculated with Eq. (5.2) and Eqs. (5.10) through (5.12) for $S=1$ using a simultaneous fit for both curves.

The critical flicker frequency can be derived from the temporal contrast sensitivity function. From the temporal contrast sensitivity function, the frequency can be calculated where the contrast sensitivity has a value 1 . At this frequency a modulation of $100 \%$ is needed to observe a luminance variation with a probability of $50 \%$ (or with a probability of $75 \%$ in a 2 AFC experiment). Extensive measurements of the critical flicker frequency have been made by Tyler \& Hamer (1990) who found an accurate match with the Ferry-Porter law over a large range of retinal illuminance levels. Fig. 5.17 shows their measurement results for a circular field with a diameter of $0.5^{\circ}$ and $0.05^{\circ}$ with a $100 \%$ modulated sinusoidal luminance variation. Viewing was monocular. The observer was $\mathrm{RDH}$, the second author. The curves through the data points have been calculated with Eq. (5.2) and Eqs. (5.10) through (5.12) for the situation that $S=1$. The values of $\tau_{10}$ and $\tau_{20}$ were $31 \mathrm{msec}$ and $18 \mathrm{msec}$, respectively, and the values used for $\eta$ and $k$ were $3 \%$ and 3.0, respectively. These values are about equal to the typical values of these constants. The agreement between measurements and calculations is very good.

The given expressions can also be applied to calculate the critical flicker frequency for the practical situation of images displayed on CRTs (cathode ray tubes). In this situation, viewing is binocular and the luminance variation is not sinusoidal, but consists of a continuously repeated exponential decay with a repetition frequency equal to the frame rate. For a non-sinusoidal luminance variation, the first harmonic of the variation has to be used to determine the visibility of flicker. The 
decay time of the luminance is usually short with respect to the repetition time of the signal. For this situation, the amplitude of the first harmonic is nearly twice the average value of the luminance. This would mean that the modulation is nearly $200 \%$, instead of $100 \%$, and that the contrast sensitivity to be used for the calculation of the critical flicker frequency is 0.5 , instead of 1 . This situation would, however, only occur if the light emission starts simultaneously in all the points of the image. This is, however, not so. In practice, the successive start of the light emission in the different points of the complete image causes an effectively flat time dependence of the luminance, except for the complete darkness of the image during the vertical retracing. This causes that the actual luminance variation has a rectangular shape. Fig. 5.18 shows a sketch of this idealized rectangular luminance variation. The first harmonic of this pattern can be considered as the cause of flicker. The modulation of this harmonic can be calculated with the aid of a Fourier analysis. For a rectangular variation holds

$$
m_{1}=\frac{2 \sin (\alpha \pi)}{\alpha \pi}
$$

where $m_{1}$ is the modulation of the first harmonic and $\alpha$ is the relative length of the rectangle. For television and computer display, the vertical ray tracing takes generally about $8 \%$ of the frame time, so that $\alpha$ is 0.92 . From Eq. (5.13) follows that the modulation of the fundamental wave is then $17.2 \%$, which means that for $S$ a value of $1 / 0.172=5.81$ has to be used to calculate the critical flicker frequency. With this value of $S$, the critical flicker frequency has been calculated as a function of the luminance with the aid of Eq. (5.2) and Eqs. (5.10) through (5.12). In this calcula-

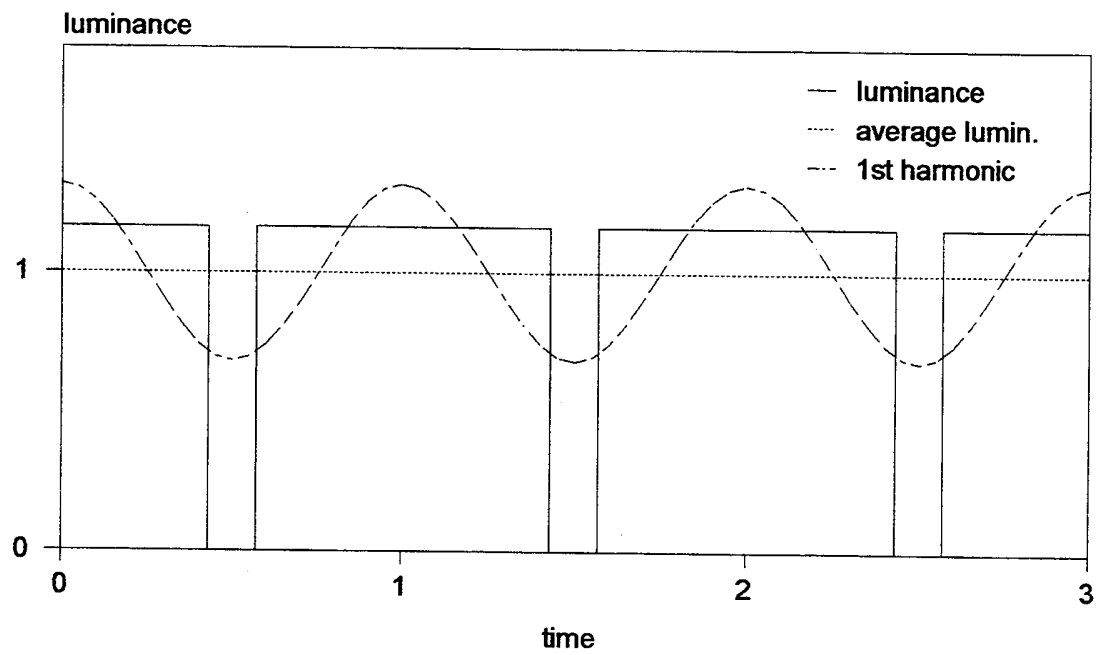

Figure 5.18: Solid curve: idealized temporal luminance variation of CRT images. Dotted curve: average luminance. Dashed curve: first harmonic of the temporal luminance variation. 


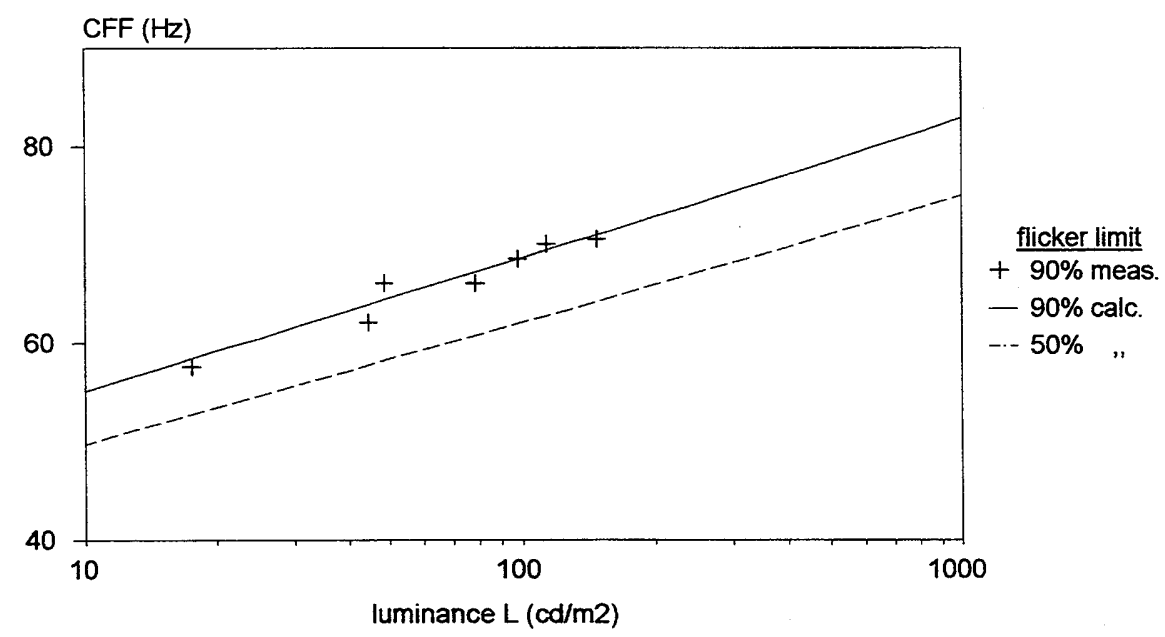

Figure 5.19: Critical flicker frequency as a function of the luminance for a CRT image seen with a subtended angle of $30^{\circ}$. Data points: CFF measurements by Farell et al. (1987) of the $90 \%$ flicker limit that corresponds with a chance of $10 \%$ for seeing flicker. Solid curve: calculation of the $90 \%$ limit with the here given model. Dashed curve: same calculation for $50 \%$ probability of seeing flicker.

tion a circular field with a diameter of $30^{\circ}$ was used, which corresponds with the average viewing situation for computer displays. Further, normal viewing conditions were assumed. For $\tau_{10}$ and $\tau_{20}$ the values $32 \mathrm{msec}$ and $18 \mathrm{msec}$, respectively, were used and for $\eta$, and $k$ the values $3 \%$ and 3.0, respectively, being the typical values of these constants. Besides the normal situation of $50 \%$ correct response, also a calculation was made for $10 \%$ correct response, which corresponds with a probability of $90 \%$ for not seeing flicker. This calculation was made by using a different value for $k$. The $k$ value for this condition is 1.72 , instead of 3.0 , as can be calculated with the aid of Eqs. (2.2) through (2.4) given in Chapter 2. The results are shown in Fig. 5.19. This figure also shows measurements by Farrell et al. (1987) of the $90 \%$ flicker limit for a CRT display seen with a subtended angle of $30^{\circ}$. This limit corresponds with a probability of $90 \%$ for not seeing flicker. The data were derived by Farrell from the mean and the standard deviation of the flicker thresholds observed by 20 observers. These data correspond very well with the calculated curve for this limit. The slope of the curve is about $14 \mathrm{~Hz}$ per decade. From the figure, it can further be seen that for a luminance level of $100 \mathrm{~cd} / \mathrm{m}^{2}$, the flicker is still visible with a probability of $50 \%$ at a frame rate of $62 \mathrm{~Hz}$. At $68 \mathrm{~Hz}$ the probability of seeing flicker is reduced to $10 \%$. From practical experience is known that the choice of a frame rate of $72 \mathrm{~Hz}$ for computer displays is sufficient to avoid flicker completely. 


\subsection{Temporal impulse response}

In the previous sections only the temporal frequency effects of the extended contrast sensitivity model were treated. The temporal impulse response function given by the model can be derived from the following equation that corresponds with Eq. (5.1):

$$
h(t)=h_{1}(t) \star\left\{1-F(u) h_{2}(t)\right\}
$$

where $h_{1}(t)$ is the unit impulse response of the temporal processing that the signal undergoes on its way from the photo-receptors to the brain, $h_{2}(t)$ is the unit impulse response of the temporal filtering of the inhibition signal, $F(u)$ is the MTF of the lowpass filter used for the lateral inhibition given by Eq. (3.21), and the symbol $\star$ denotes convolution. This equation can also be written in the form

$$
h(t)=h_{1}(t)-F(u)\left\{h_{1}(t) \star h_{2}(t)\right\}
$$

The functions $h_{1}(t)$ and $h_{2}(t)$ are the inverse Fourier transforms of the complex functions of which the functions $H_{1}(w)$ and $H_{2}(w)$, respectively, represent the absolute value. As was already mentioned in section 5.3, the functions $H_{1}(w)$ and $H_{2}(w)$ contain only amplitude information and not the required phase information, so that the impulse response function cannot be found by an inverse Fourier transform of these functions. However, if it is here assumed that the impulse response of these functions is simply given by Eq. (5.4), so that the total temporal impulse response function can be calculated by using this equation for $h_{1}(t)$ and $h_{2}(t)$ in Eq. (5.15). For a short pulse, the so obtained impulse response function has a triphasic shape. This means, that it starts with a negative part, followed by a positive part and ending again with a negative part, as is shown in Fig. 5.20. The negative part is caused by the second term in Eq. (5.15) which represents the lateral inhibition. The shape of this function is different from the biphasic shape consisting of a positive part followed by a negative part, which is generally assumed in other models. See Watson (1982).

At present no biological measurements of the impulse response function are available yet. However, Roufs \& Blommaert (1981) developed, a sophisticated psychophysical method to measure this function indirectly. They used a probe flash and a test flash with a very short duration compared with the duration of the impulse response. The duration of both flashes was $2 \mathrm{msec}$. Both flashes were superimposed on a constant luminance level. The test flash has a much smaller intensity than the probe flash and proceeds the probe flash, or is delayed with respect to it, by a variable time difference. At each time difference, the intensity of both flashes is varied with the same factor until the combined intensity of probe and test flash is just observed. The impulse response function is derived from the intensities of the flashes, the time difference between the flashes, and the threshold of the probe flash without test flash. This technique is similar to the technique developed by the same authors for the measurement of the spatial point-spread function, which was mentioned in section 


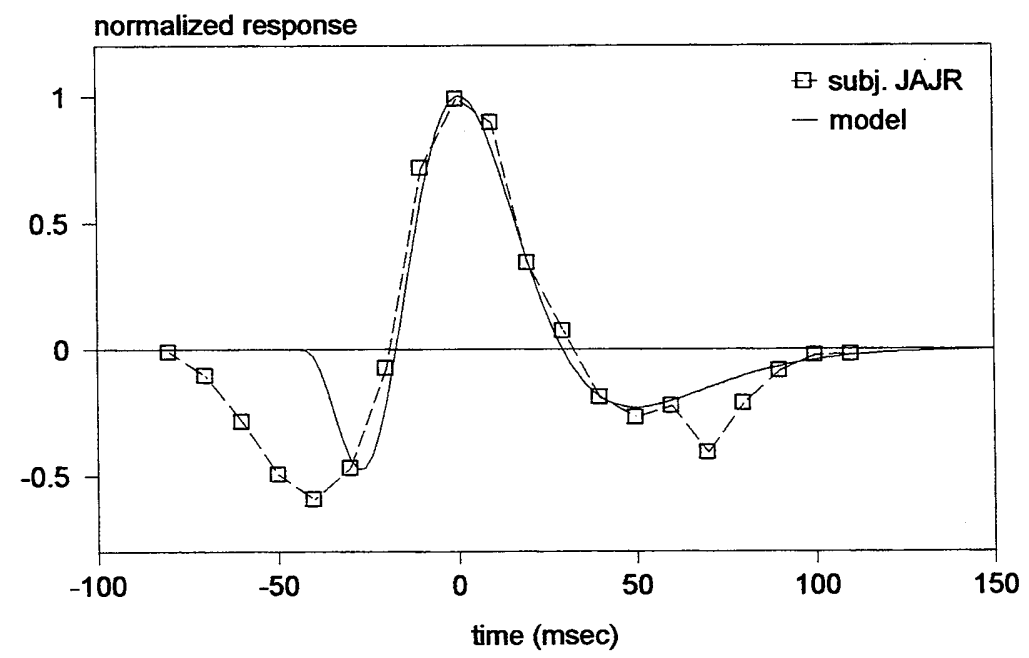

Figure 5.20: Temporal impulse response function measured by Blommaert and Roufs (1987). The solid curve has been calculated with Eqs. (5.18) and (5.7).

\section{6 of Chapter 3.}

Fig. 5.20 shows the measurements results given by Blommaert \& Roufs (1987) for subject JAJR, the second author. The measurements were made with a circular field with a diameter of $1^{\circ}$ and a retinal illuminance of $1200 \mathrm{Td}$. The data points are the average of two measurement series. As the method gives no information about the zero point of the time scale, the time scale was arbitrarily set to zero at the maximum response. Furthermore, the response was arbitrarily set to 1 at this maximum. The solid curve in the figure has been calculated with Eqs. (5.15) and (5.4) with $\tau_{1}=$ $7.2 \mathrm{~ms}$ and $\tau_{2}=13.5 \mathrm{msec}$, being the values that were obtained with the measurements of Fig. 5.6, which were made with the same subject and under the same conditions regarding field size and illuminance. $F(u)$ was calculated with Eqs. (3.21) and (5.10) that give for these measurements a value of 0.93 . The agreement of the calculated curve with the measurements shows that the use of Eq. (5.4) is indeed correct and that the use of this function together with Eq. (5.15) gives a good description of the temporal response function of the eye. Note that in Fig. 5.19 the same values for the time constants were used as in Fig. 5.6. It must be remarked that the calculations show a disagreement with the measurements in the first negative part of the curve. The total area under the negative part of the temporal response function must theoretically be nearly equal to the positive part. For the measurements, the area under the negative part is much larger then follows from the ratio $0.93: 1$ used for the calculations. This means that the negative inhibition would be stronger than the positive excitation. As this is unlikely, it must be assumed that this deviation has probably some other cause. 
Watson (1982) mentioned in a short paper that the results of the measurements by Roufs and Blommaert need not to be equal to the temporal response function and that a temporal response function with a biphasic shape could also explain their results. However, a triphasic shape was later also found by Tyler (1992) with a different measurement technique. It should further be remarked that the triphasic shape has a positive effect on the observation of temporal signals by the eye. With a triphasic shape, a negative part of the temporal response function precedes the main positive part. This gives a sharpening effect of temporal luminance changes, similar to the sharpening effect in the space domain known as Mach-band effect. Both effects are caused by the lateral inhibition and both effects improve the possibility of the eye to observe luminance changes.

\subsection{Summary and conclusions}

In this chapter the spatial contrast sensitivity model described in Chapter 3 has been extended to the temporal domain so that it can also be used for the temporal contrast sensitivity. The extension is based on the assumption that the lateral inhibition signal undergoes temporal filtering in addition to spatial filtering. With the so obtained spatiotemporal model, the remarkable spatiotemporal behaviour of the visual system reported in several publications can simply be explained. Lateral inhibition appears to play an important role in these phenomena.

In the model two different time constants are used that both depend on retinal illuminance and field size. From an analysis of published temporal contrast sensitivity measurements, an approximation formula has been derived for the dependence of the time constants on these parameters. In this way the temporal contrast sensitivity of an individual observer can be characterized by two time constants that are independent of these parameters. The so obtained model appeared to be in very good agreement with published measurements of the temporal contrast sensitivity function.

The model for the temporal contrast sensitivity function has also been used to calculate the critical flicker frequency for television and data display systems. The calculated results appeared to be in very good agreement with published measurements. The given method for the calculation of the flicker sensitivity of these systems can be used for a technical design of these systems.

The temporal contrast sensitivity function can also been used to calculate the temporal impulse response of the eye. With some additional assumption, the so obtained impulse response function appeared to have a triphasic shape. This shape is different from the biphasic shape obtained with other models. Although no biological measurements of the temporal impulse response function are available yet, the 
prediction obtained with the model appeared to be in good agreement with psychophysical measurements by Roufs and Blommaert.

\section{References}

Barten, P.G.J. (1993). Spatio-temporal model for the contrast sensitivity of the human eye and its temporal aspects. Human Vision, Visual Processing, and Digital Display IV, Proc. SPIE, 1913,2-14.

Blommaert, F.J.J. \& Roufs, J.A.J. (1987). Predictions of thresholds and latency on the basis of experimentally determined impulse responses. Biological Cybernetics, $56,329-344$.

Burbeck, C.A. \& Kelly, D.H. (1980). Spatiotemporal characteristics of visual mechanisms: excitatory-inhibitory model. Journal of the Optical Society of America A, 70, $1121-1126$.

Campbell, F.W. \& Robson, J.G. (1968). Application of Fourier analysis to the visibility of gratings. Journal of Physiology, 197, 551-566.

Daly, S.J. \& Normann, R.A. (1985). Temporal information processing in cones: effects of light adaptation on temporal summation and modulation. Vision Research, 25, 1197-1206.

Farrell, J.E., Benson, B.E., and Haynie, C.R. (1987). Predicting flicker thresholds for visual displays. Proceedings of the SID, 28, 449-453.

Ferry, E.S. (1892). Persistence in vision. American Journal of Science, 44, 192-207.

Fleet, D.J., Hallett, P.E., and Jepson, A.D. (1985). Spatiotemporal inseparability in early visual processing. Biological Cybernetics, 52, 153-164.

Kelly, D.H. (1960). Jo stimulus patterns for visual research. Journal of the Optical Society of America, 50, 111 5-11 16.

Kelly, D.H. (1961). Visual responses to time-dependent stimuli. I. Amplitude sensitivity measurements. Journal of the Optical Society of America, 51, 422-429.

Kelly, D.H. (1971). Theory of flicker and transient responses, II: counterphase gratings. Journal of the Optical Society of America, 61, 632-640.

Kelly, D.H. (1972). Adaptation effects on spatio-temporal sine-wave thresholds. Vision Research, 12, 89-101.

Kelly, D.H. (1979). Motion and vision, II: stabilized spatio-temporal threshold surface. Journal of the Optical Society of America, 69, 1340-1349.

Koenderink, J.J. \& van Doorn A.J. (1979). Spatiotemporal contrast detection threshold surface is bimodal. Optics Letters, 4, 32-34.

Kulikowski, J.J. \& Tolhurst, D.J. (1973). Psychophysical evidence for sustained and 
transient detectors in human vision. Joumal of Physiology, 232, 149-162.

de Lange, H. (1952). Experiments on flicker and some calculations on an electrical analogue of the foveal systems. Physica, 18, 935-950.

de Lange, H. (1954). Relationship between critical flicker-frequency and a set of low-frequency characteristics of the eye. Journal of the Optical Society of America, 44, 380-389.

de Lange, H. (1957). Attenuation characteristics and phase-shift characteristics of the human fovea-cortex systems in relation to flicker fusion phenomena. $\mathrm{Ph}$. $\mathrm{D}$. Thesis, Delft Technical University, Delft, The Netherlands.

de Lange, H. (1958a). Research into the dynamic nature of the human fovea-cortex systems with intermittent and modulated light. I. Attenuation characteristics with white and colored light. Journal of the Optical Society of America, 48, 777-784.

de Lange, $H$. (1958b). Research into the dynamic nature of the human fovea-cortex systems with intermittent and modulated light. II. Phase shift in brightness and delay in color perception. Journal of the Optical Society of America, 48, 784-789.

de Lange, H. (1961). Eye's response at flicker fusion to square-wave modulation of a test field surrounded by a large field of equal mean luminance. Journal of the Optical Society of America, 51, 415-421.

van Nes, F.L., Koenderink, J.J., Nas, H., and Bouman, M.A. (1967). Spatiotemporal modulation transfer in the human eye. Journal of the Optical Society of America, 57, 1082-1088.

Porter, T.C. (1902). Contributions to the study of flicker. Proceedings of the Royal Society, London A, 70, 313-329.

Robson, J.G. (1966). Spatial and temporal contrast-sensitivity functions of the visual system. Journal of the Optical Society of America, 56, 1 1417-1 142.

Roufs, J.A.J. (1972a). Dynamic properties of vision-I. Experimental relationships between flicker and flash thresholds. Vision Research, 12, 261-278.

Roufs, J.A.J. (1972b). Dynamic properties of vision-II. Theoretical relationships between flicker and flash thresholds. Vision Research, 12, 279-292.

Roufs, J.A.J. (1973). Dynamic properties of vision-III. Twin flashes, single flashes and flicker fusion. Vision Research, 13, 309-323.

Roufs, J.A.J. (1974a). Dynamic properties of vision-IV. Thresholds of decremental flashes, incremental flashes and doublets in relation to flicker fusion. Vision Research, 14, 831-852.

Roufs, J.A.J. (1974b). Dynamic properties of vision-V. Perception lag and reaction time in relation to flicker and flash thresholds. Vision Research, 14, 853-869.

Roufs, J.A.J. (1974c). Dynamic properties of vision-IV. Thresholds of decremental flashes, incremental flashes and doublets in relation to flicker fusion. Vision 
Research, 14, 831-852.

Roufs, J.A.J. \& Blommaert, F.J.J. (1981). Temporal impulse and step responses of the human eye obtained psychophysically by means of a drift-correcting perturbation technique. Vision Research, 21, 1203-1221.

Roufs, J.A.J. \& Bouma, H. (1980). Towards linking perception research and image quality. Proceedings SID, 21, 247-270.

Swanson, W.H., Ueno, T., Smith, V.C., and Pokorny, J. (1987). Temporal modulation sensitivity and pulse-detection thresholds for chromatic and luminance perturbations. Journal of the Optical Society of America A, 4, 1992-2005.

Tyler, C.W. (1989). Two processes control variations in flicker sensitivity over the life span. Journal of the Optical Society of America A, 6, 481-490.

Tyler, C.W. (1992). Psychophysical derivation of the impulse response through generation of ultrabrief responses: complex inverse estimation without minimum-phase assumptions. Journal of the Optical Society of America A, 9, 10251040.

Tyler, C.W. \& Hamer, R.D. (1990). Analysis of visual modulation sensitivity. IV. Validity of the Ferry-Porter law. Journal of the Optical Society of America A, 7, 743-758.

Watson, A.B. (1982) Derivation of the impulse response: comments on the method of Roufs and Blommaert. Vision Research, 22, 1335-1337.

Watson, A.B. (1986). Temporal sensitivity. In: K.R. Boff, L. Kaufman, and J.P. Thomas (Eds.) Handbook of Perception and Human Performance, I. Sensory Processes and Perception, chapter 6. Wiley, New York. 


\section{Chapter 6}

\section{Effect of nonwhite spatial noise on contrast sensitivity}

\subsection{Introduction}

In Chapter 2 where the effect of external noise on contrast sensitivity has been treated, it was assumed that the noise was white. This means that the spectral density of the noise is constant within the frequency limits of the considered spectrum. Although these conditions are usually met, this is not always the case. Sometimes the spectral density is not constant within the frequency limits of the observed spectrum or is constant only within a limited part of this spectrum. The disturbance of the observation of a signal by noise with frequencies that are different from that of the signal is called masking. In this chapter the effect of nonwhite noise on contrast sensitivity will be treated. It will be investigated how the formulae for the effect of white noise given in Chapter 2 can be generalized to become also valid for the situation of nonwhite noise. This treatment will, however, be restricted to spatial noise. If also temporal noise is present, it will be assumed that this noise is white.

\subsection{Model for the masking effect of nonwhite spatial noise}

For white noise, the threshold elevation of a signal by the presence of external noise is given by Eq. (2.50) in Chapter 2:

$$
m_{\mathrm{t}}^{\prime}=\sqrt{m_{\mathrm{t}}^{2}+k^{2} m_{\mathrm{n}}^{2}}
$$

where $m_{\mathrm{t}}$ is the modulation threshold without noise, $m_{\mathrm{t}}{ }_{\mathrm{t}}$ is the increased modulation threshold with noise, and $m_{\mathrm{n}}$ is the average modulation of the noise wave components given by Eq. (2.43): 


$$
m_{\mathrm{n}}=2 \sqrt{\frac{\Phi_{\mathrm{n}}}{X Y T}}
$$

For white noise, $\Phi_{n}$ and $m_{n}$ are constant over the whole frequency spectrum of the noise band. For nonwhite noise, the spectral density of the noise will vary with the spatial frequency $u_{\mathrm{n}}$ of the noise and $m_{\mathrm{n}}$ will therefore be different for different spatial frequencies $u$ of the signal. To generalize the equations for white noise, so that they can also be used for nonwhite noise, it is assumed here that $m_{\mathrm{n}}$ can be obtained by the following equation (Barten, 1995):

$$
m_{\mathrm{n}}(u)=2 \sqrt{\frac{\Phi_{\mathrm{d}}(u)}{X Y T}}
$$

This expression is similar as Eq. (2.43), but $\Phi_{\mathrm{n}}$ has been replaced by the function $\Phi_{\mathrm{d}}(u)$. This function describes the equivalent effect of the different spatial frequency components of the noise on the signal at spatial frequency $u$. For white noise, the function $\Phi_{d}(u)$ is simply equal to $\Phi_{n}$. For nonwhite noise, it is assumed that $\Phi_{d}(u)$ can be derived from the frequency spectrum of the noise with the aid of the following linear relation:

$$
\Phi_{\mathrm{d}}(u)=\int_{0}^{\infty} \Psi\left(u, u_{\mathrm{n}}\right) \Phi_{\mathrm{n}}\left(u_{\mathrm{n}}\right) \frac{\mathrm{d} u_{\mathrm{n}}}{u}
$$

where $u_{n}$ is the spatial frequency of the noise and $\Psi\left(u, u_{n}\right)$ is a dimensionless weighting function that describes the masking effect of noise wave components with spatial frequency $u_{n}$ on the observation of a signal with spatial frequency $u$.

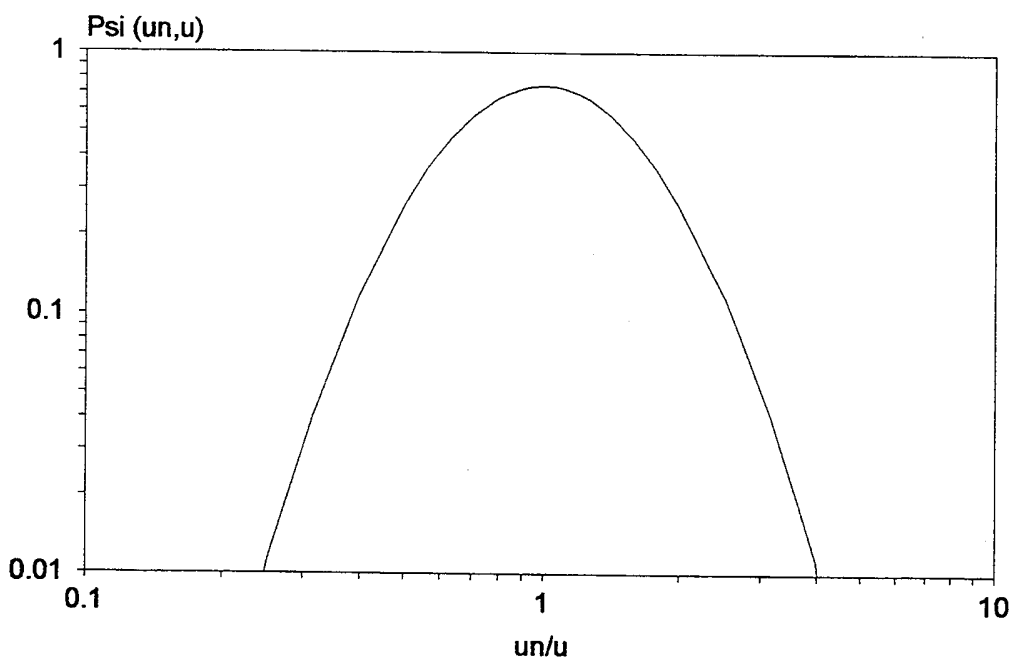

Figure 6.1: Plot on double logarithmic scale of the function $\Psi\left(u, u_{n}\right)$ given by Eq. (6.4) which describes the masking effect of noise components with spatial frequency $u_{n}$ on a signal with spatial frequency $u$. 
To obtain that $\Phi_{d}(u)$ is equal to $\Phi_{n}$ for white noise, the function $\Psi\left(u, u_{n}\right)$ has to meet the following condition:

$$
\int_{0}^{\infty} \Psi\left(u, u_{\mathrm{n}}\right) \frac{\mathrm{d} u_{\mathrm{n}}}{u}=1
$$

The function $\Psi\left(u, u_{n}\right)$ will generally be zero for most values of $u_{n}$ and will differ from zero only for a range of values around $u$. Furthermore, $\Psi\left(u, u_{n}\right)$ appears to be a function of $u_{\mathrm{r}} / u$. From measurements by Stromeyer $\&$ Julesz (1972) about masking effects by nonwhite noise, which will be treated in the next section, the following empirical relation can be derived:

$$
\Psi\left(u, u_{\mathrm{n}}\right)=0.747 \mathrm{e}^{-2.2 \ln ^{2}\left(u_{\mathrm{n}} / u\right)}
$$

This expression has been obtained from the data after trying various other expressions to get a good fit with the data. The factor 0.747 has been chosen such that the condition of Eq. (6.3) is met. The function is shown in Fig. 6.1. It is a kind of Gaussian distribution function with a $50 \%$ width of nearly two octaves.

A special situation of nonwhite noise occurs when the spectral density of the noise is constant, but the frequency band of the noise is outside the spatial frequency of the signal. This situation is shown in Fig. 6.2. Then Eq. (6.2) can be simplified to

$$
\Phi_{\mathrm{d}}(u)=\Phi_{\mathrm{n}} \int_{u_{\min }}^{u_{\max }} \Psi\left(u, u_{\mathrm{n}}\right) \frac{\mathrm{d} u_{\mathrm{n}}}{u}
$$

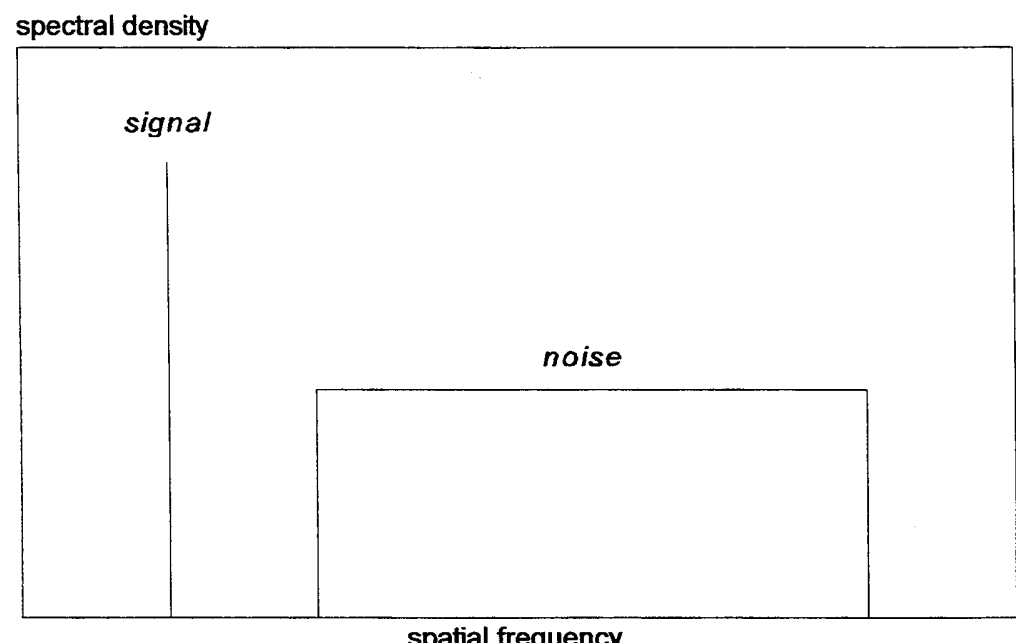

spatial frequency

Figure 6.2: Noise spectrum for the situation of one-dimensional white noise in a spatial frequency band outside the spatial frequency of the signal. 
where $u_{\min }$ and $u_{\max }$ are the minimum and maximum spatial frequency of the noise, respectively. Often the relative standard deviation $\sigma_{n}$ of the noise is given, instead of the spectral density. Then Eq. (2.41) of Chapter 2 can be used to calculate $\Phi_{n}$.

\subsection{Measurements with narrow noise bands by Stromeyer and Julesz}

Stromeyer \& Julesz (1972) measured the threshold elevation of a large series of spatial frequencies using a one-dimensional vertically oriented dynamic noise pattern of which the spatial frequency range of the noise band was varied. The spectral density was constant within the noise band. The edges of the bands had a steepness of $42 \mathrm{~dB} /$ octave and their position was determined by measuring the $-3 \mathrm{~dB}$ points. Noise intensity was determined by measuring $\sigma_{\mathrm{n}}$. The stimuli were vertically oriented sinusoidal gratings displayed on a high-resolution monitor provided with a white phosphor (P4). The frame rate was $60 \mathrm{~Hz}$ and the average luminance was $15.9 \mathrm{~cd} / \mathrm{m}^{2}$. In most of the experiments the viewing distance was $4 \mathrm{~m}$ and the stimulus field was $2.5^{\circ} \times 1^{\circ}$. The test field was surrounded by a dark area. Viewing was binocular with a natural pupil. The modulation threshold was determined by the method of adjustment. Two subjects out of three (MHW, RAP, and CFS, the first author) served as observer in different parts of the experiments. For the evaluation of the data, the average of the results of these two observers was used to reduce the experimental spread.

For the one-dimensional dynamic noise used in these experiments, Eq. (2.41) of Chapter 2 gives

$$
\Phi_{\mathrm{n}}=\frac{\sigma_{\mathrm{n}}^{2}}{2\left(u_{\max }-u_{\min }\right) 2 w_{\max }}
$$

so that Eq. (6.5) becomes

$$
\Phi_{\mathrm{d}}(u)=\frac{\sigma_{\mathrm{n}}^{2}}{2\left(u_{\max }-u_{\min }\right) 2 w_{\max }} \int_{u_{\min }}^{u_{\max }} \Psi\left(u, u_{\mathrm{n}}\right) \frac{\mathrm{d} u_{\mathrm{n}}}{u}
$$

For one-dimensional dynamic noise Eq. (6.1) becomes

$$
m_{n}(u)=2 \sqrt{\frac{\Phi_{\mathrm{d}}(u)}{X T}}
$$

The authors expressed the measurement results as a relative threshold elevation defined by 


$$
l=\frac{m_{\mathrm{t}}^{\prime}}{m_{\mathrm{t}}}-1
$$

From this relation follows with the aid of Eq. (2.50) of Chapter 2

$$
l=\sqrt{\frac{m_{\mathrm{n}}^{2}(u)}{\left(m_{\mathrm{t}} / k\right)^{2}}+1}-1
$$

In this expression $m_{\mathrm{t}} / k$ can be calculated with Eq. (3.26). The actual value of $k$ is irrelevant, because $m_{\mathrm{t}}$ is proportional with $k$.

With these equations the relative threshold elevation $l$ was calculated for the measurement conditions of the experiment, using Eq. (6.4) for the function $\Psi\left(u, u_{n}\right)$. Figs. 6.3 and 6.4 show the measurement data and the calculations for five different one-octave wide noise bands. The measurements for the lowest noise band in Fig. 6.3 were made at a viewing distance of $0.5 \mathrm{~m}$ with a field size of $20^{\circ} \times 8^{\circ}$, whereas the measurements for the lowest noise band in Fig. 6.4 were made at a viewing distance of $1 \mathrm{~m}$ with a field size of $10^{\circ} \times 4^{\circ}$. The other measurements in both figures were made at a viewing distance of $4 \mathrm{~m}$ with a field size of $2.5^{\circ} \times 1^{\circ}$. For most of the measurements the value of $\sigma_{n}$ was 0.059 except the lowest and the highest noise bands in Fig. 6.3 for which $\sigma_{n}$ was 0.042 and 0.047 , respectively. Subjects MHW and CFS were the observers. The average of their measurement result was used in the figures. The values of $\sigma_{0}$ and $\eta$ used for the calculation were 0.5 arc min and $4 \%$, respectively.

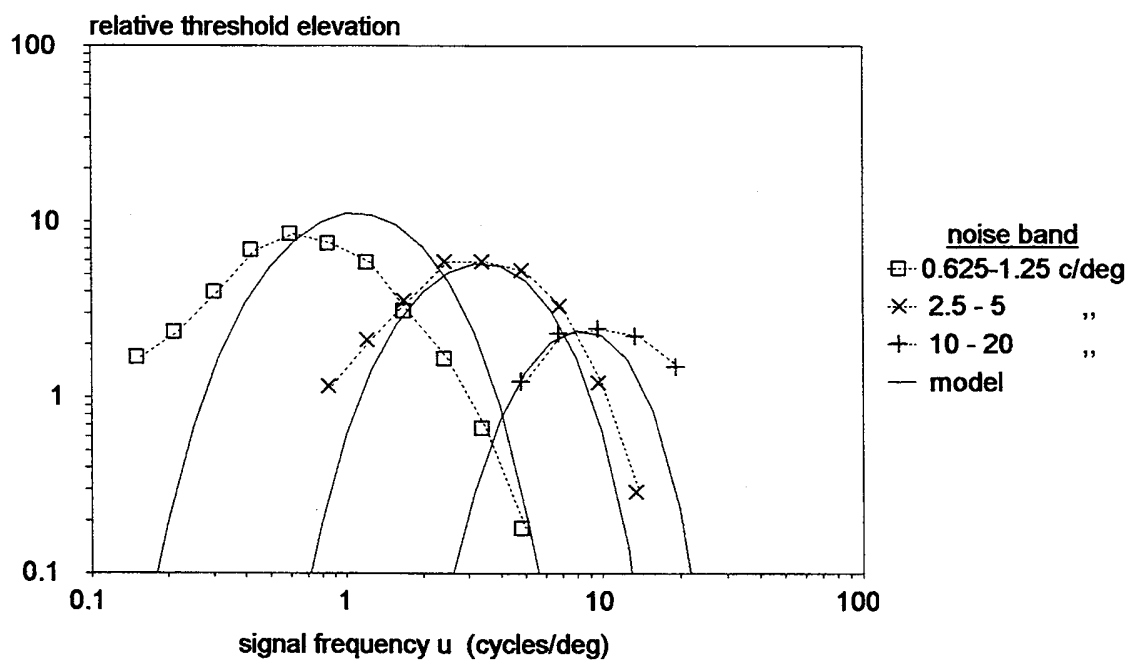

Figure 6.3: Relative threshold elevation by one-octave wide noise bands measured by Stromeyer \& Julesz (1972). Luminance $15.9 \mathrm{~cd} / \mathrm{m}^{2}$. Binocular viewing with a natural pupil. The solid curves have been calculated with Eqs. (6.7) through (6.10) and Eqs. (3.26) and (6.4). 


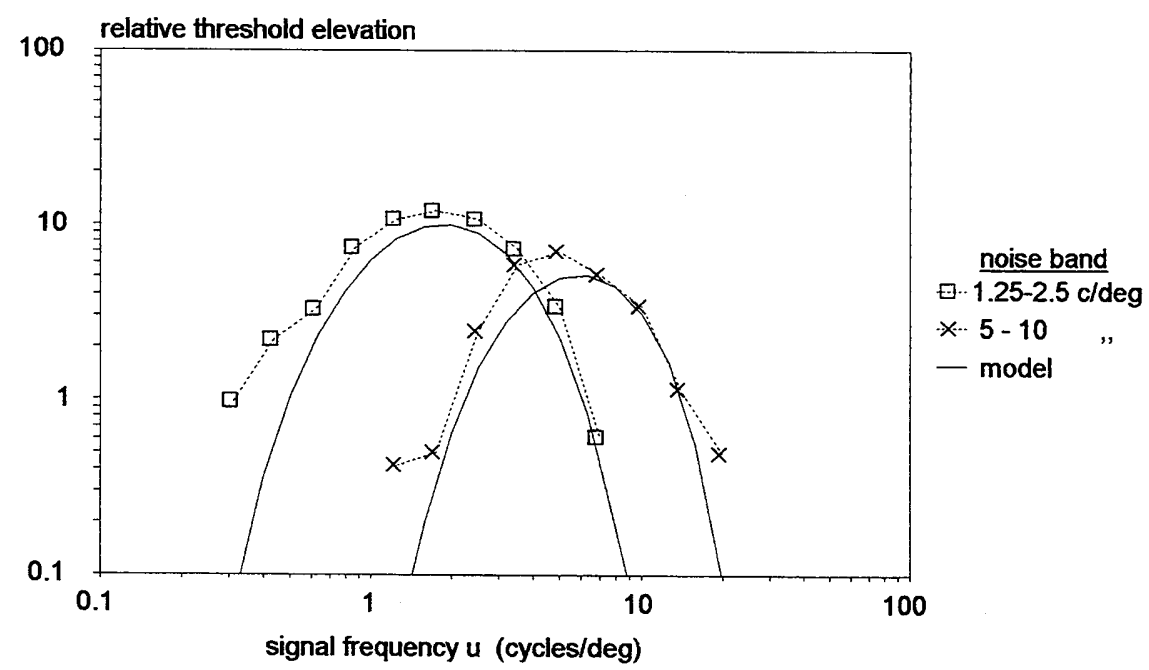

Figure 6.4: Same as Fig. 6.3 for two other one-octave wide noise bands.

Apart from measurements with narrow noise bands, Stromeyer and Julesz also made measurements with wide noise bands of variable size. Fig. 6.5 shows the threshold elevation by lowpass noise as a function of the maximum spatial frequency of the noise for three different spatial frequencies of the test signal. In this experiment

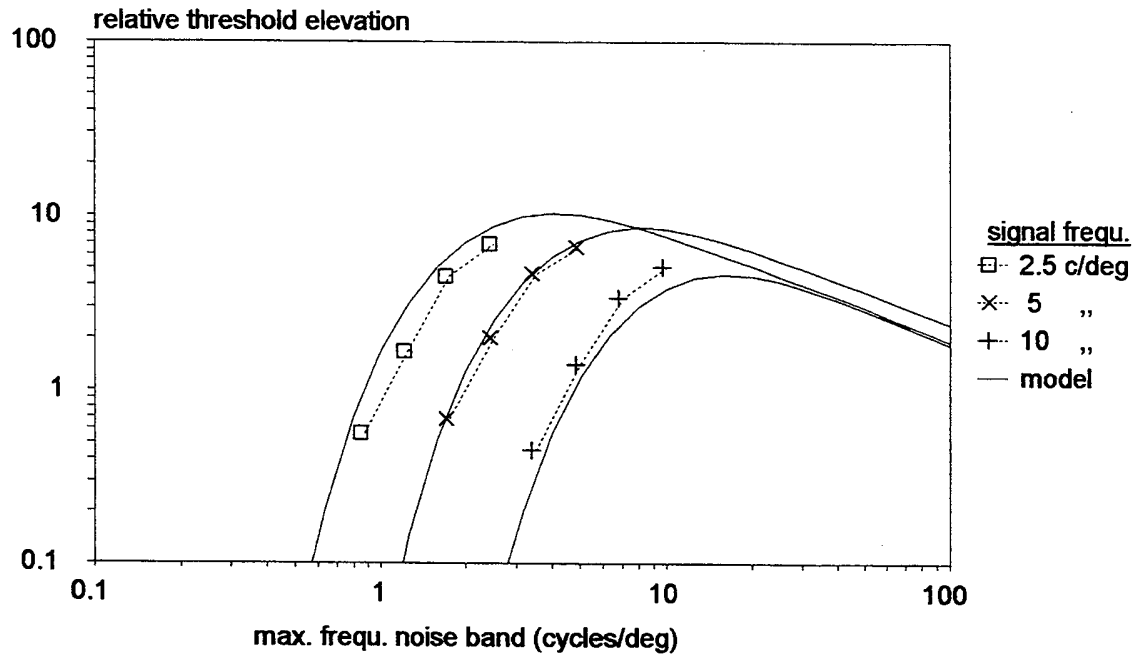

Figure 6.5: Relative threshold elevation by lowpass noise measured by Stromeyer \& Julesz (1972) as a function of the maximum frequency of the noise band for three different frequencies of the test signal. Luminance $5.19 \mathrm{~cd} / \mathrm{m}^{2}$. Binocular viewing with a natural pupil. The solid curves have been calculated with Eqs. (6.7) through (6.10) and Eqs. (3.26) and (6.4). 
$\sigma_{n}$ was maintained at a fixed value of 0.15 during the variation of the maximum frequency of the noise. The constant value of $\sigma_{n}$ causes a decrease of spectral noise density with increasing maximum frequency of the noise. This explains the decrease of the threshold elevation at the right-hand side of the curves. Subjects RAP and CFS were the observers. The average of their measurement results has been used in the figure. The solid curves have been calculated in the same way as for the measurements in Figs. 6.3 and 6.4. The values of $\sigma_{0}$ and $\eta$ used for the calculation were 0.5 arc min and $1.2 \%$, respectively. The calculated curves in Figs. 6.3 through 6.5 show that by using Eq. (6.4) for the function $\Psi\left(u, u_{n}\right)$ on the average a good agreement with the measurements can be obtained.

\subsection{Measurements with nonwhite noise by van Meeteren and Valeton}

To test if the function given by Eq. (6.4) is also valid for other measurements, an analysis has been made of measurements van Meeteren \& Valeton (1988). They measured the contrast sensitivity function without noise and with three types of nonwhite two-dimensional static noise that differed in bandwidth. Vertically oriented sinusoidal grating patterns with and without noise were generated on the screen of a video monitor. The patterns had a luminance of $100 \mathrm{~cd} / \mathrm{m}^{2}$ and were surrounded by a large field with the same luminance. They were observed from a distance of $3.5 \mathrm{~m}$. The field size was $1^{\circ} \times 1^{\circ}$. Viewing was binocular with a natural pupil. Two subjects with normal visual acuity (AVM and MV, the authors) took part in the experiments. The modulation threshold was determined by measurement of the psychometric function. The displayed picture consisted of $180 \times 180$ pixels. Random noise was generated with a computer in each pixel. In this way fine-grained noise was made. Apart from this fine-grained noise, medium-grained noise and coarse-grained noise was generated by assigning random luminance values to a rectangular grid of pixels. The spacing of this grid was 5 pixels and 20 pixels, respectively, whereas the luminance value of the remaining pixels was interpolated by using a first-order Bessel function. In this way the maximum spatial frequencies of the noise bands were 90 cycles/deg, 18 cycles/deg, and 4.5 cycles/deg in both directions for the three types of noise, respectively. The minimum spatial frequency of the noise band was 0.5 cycles/deg for the fine-grained noise, as follows from the size of the picture, but appeared to be 2 cycles/deg for the two other types of noise. The value of $\sigma_{n}$ was 0.45 for fine-grained noise and 0.22 for the two other types of noise. These last two values have to be multiplied with $\sqrt{ }(4 / \pi)$ for the calculation of $\Phi_{n}$ because of the circular spatial frequency limit caused by the interpolation with the Bessel function. The large bandwidth of the fine-grained noise and the medium-grained noise makes that these types of noise may practically be considered as white noise. Only the coarse-grained 
noise is clearly nonwhite noise.

For the two-dimensional static noise used in this experiment, Eq. (2.41) of Chapter 2 gives

$$
\Phi_{\mathrm{n}}=\frac{\sigma_{\mathrm{n}}^{2}}{2\left(u_{\max }-u_{\min }\right) 2\left(v_{\max }-v_{\min }\right)}
$$

so that Eq. (6.5) becomes

$$
\Phi_{\mathrm{d}}(u)=\frac{\sigma_{\mathrm{n}}^{2}}{2\left(u_{\max }-u_{\min }\right) 2\left(v_{\max }-v_{\min }\right)} \int_{u_{\min }}^{u_{\max }} \Psi\left(u, u_{\mathrm{n}}\right) \frac{\mathrm{d} u_{\mathrm{n}}}{n}
$$

For two-dimensional static noise Eq. (6.1) becomes

$$
m_{\mathrm{n}}(u)=2 \sqrt{\frac{\Phi_{\mathrm{d}}(u)}{X Y}}
$$

Figs. 6.6 and 6.7 show the measurements of the contrast sensitivity function without noise and with the three types of noise for subject AVM and subject MV, respectively. The solid curves have been calculated with Eqs. (6.12) and (6.13) and Eqs. (3.26) and (6.4). For Fig. 6.6 the values of $\sigma_{0}, \eta$, and $k$ used for the calculation were 0.6 arc $\mathrm{min}, 0.9 \%$ and 3.2 , respectively, and for Fig. 6.7 these values were 0.45 arc min, $0.9 \%$ and 3.7 , respectively. The general agreement between measurements and calculations is good. As expected, only the coarse-grained noise condition shows

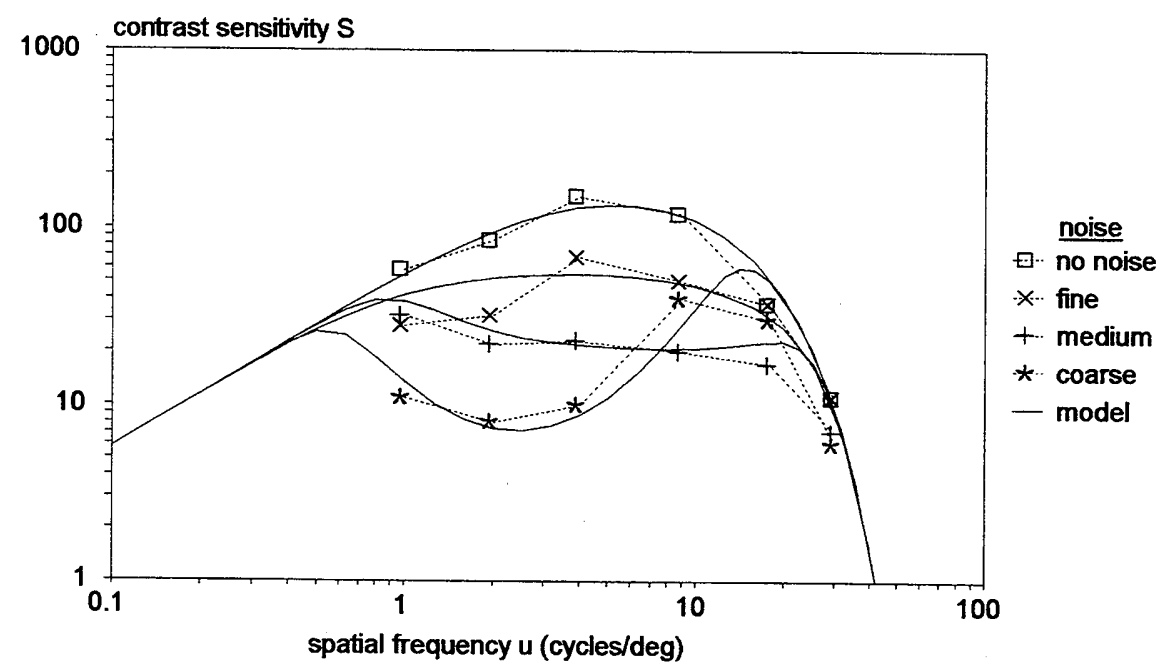

Figure 6.6: Contrast sensitivity function measured by van Meeteren \& Valeton (1988) without noise and with three types of noise that differ in bandwidth. Luminance 100 $\mathrm{cd} / \mathrm{m}^{2}$. Field size $1^{\circ} \times 1^{\circ}$. Binocular viewing with a natural pupil. Subject AVM. The solid curves have been calculated with Eqs. (6.12) and (6.13) and Eqs. (3.26) and (6.4). 


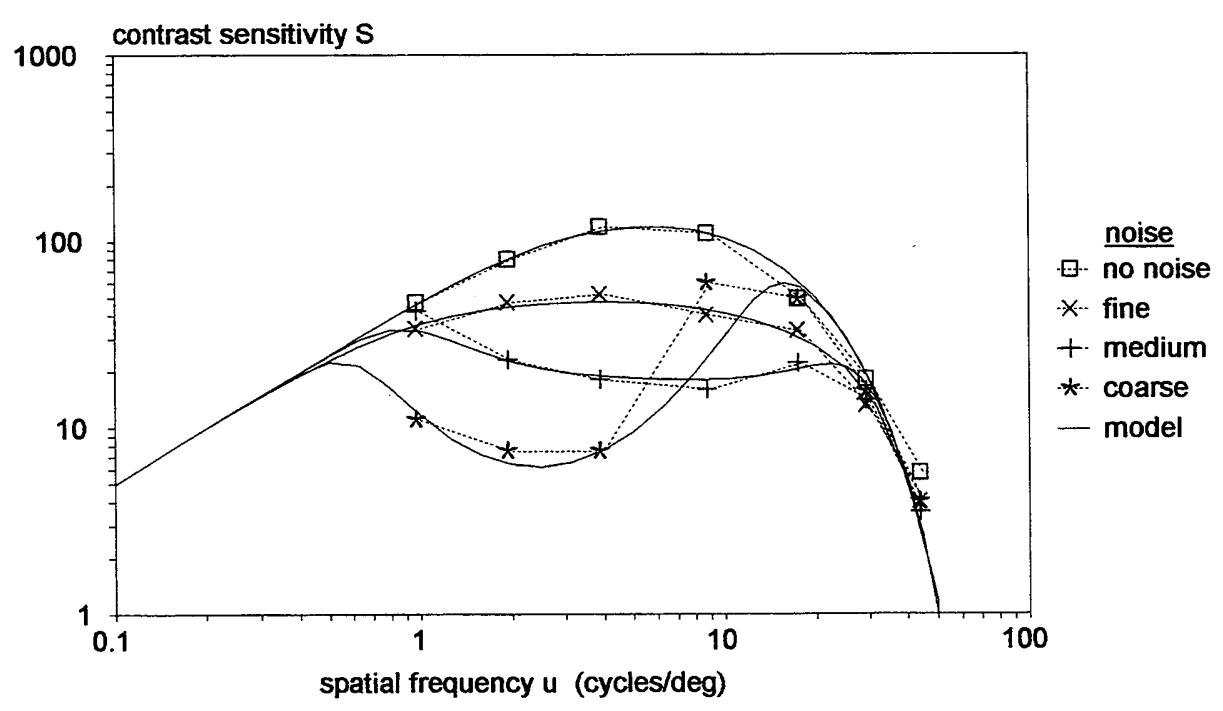

Figure 6.7: Similar as Fig. 6.6 , but for subject MV.

a clear effect of the bandwidth limitation. Although the maximum spatial frequency of this noise is 4.5 cycles/deg, the effect on the contrast sensitivity is still noticeable up to $10 \mathrm{cycles} / \mathrm{deg}$. The given formulae for nonwhite noise are principally also valid for the more simple situation of white noise. The agreement between measurements and calculations for fine-grained noise shows that this is indeed true.

\subsection{Summary and conclusions}

In this chapter a method has been given to calculate the effect of nonwhite spatial noise on contrast sensitivity. For this purpose the equations for white noise given in Chapter 2 have been generalized so that they can also be used for nonwhite noise. This was possible by the introduction of a function that describes the masking effect by noise components with one spatial frequency on the observation of a signal with a different spatial frequency. The shape of this function was derived from contrast sensitivity measurements by Stromeyer \& Julesz (1972) with narrow noise bands. This function is a log normal distribution function with a $50 \%$ width of nearly two octaves. The validity of this function has been confirmed by a comparison of the model with contrast sensitivity measurements by van Meeteren \& Valeton (1988) with and without white and nonwhite noise. These measurements also confirmed that formulae for nonwhite noise can principally also be used for white noise. 


\section{References}

Barten, P.G.J. (1995). Simple model for spatial frequency masking and contrast discrimination. Human Vision, Visual processing, and Digital Display VI, Proc. SPIE, 2411, 142-158.

van Meeteren, A \& Valeton, J. (1988). Effects of pictorial noise interfering with visual detection. Journal of the Optical Society of America A, 5, 438-444.

Stromeyer, C.F. \& Julesz, B. (1972). Spatial frequency masking in vision: critical bands and spread of masking. Journal of the Optical Society of America, 62, 12211232. 


\section{Chapter 7}

\section{Contrast discrimination model}

\subsection{Introduction}

The equations for nonwhite noise given in the previous chapter can also be used for the masking effect of one signal by the presence of a another signal. This application will be used at the development of a model for contrast discrimination that will be given in this chapter. At contrast discrimination, a difference has to be observed between two sinusoidal signals of the same luminance and spatial frequency that differ only in modulation. This situation is different from the situation at contrast detection where only a difference has to be observed between the presence and the absence of a signal. In contrast discrimination experiments one signal has a fixed modulation and the modulation of the other signal is varied until a difference in modulation is observed. The signal with the fixed modulation is called the reference signal and the signal with the variable modulation is called the test signal. Contrast detection can be considered as a form of contrast discrimination where the reference signal has zero amplitude. The contrast discrimination model gives the just-noticeable difference in modulation between two sinusoidal signals of the same spatial frequency as a function of the modulation of the reference signal.

For the development of this model first an evaluation will be made of the psychometric function occurring in contrast discrimination experiments. The psychometric function, which plays an important role at contrast detection, plays also an important role at contrast discrimination. From the psychometric function of contrast discrimination experiments, an equation will be derived for the functional parameter in the contrast discrimination process. This quantity will further be used in the evaluation of the given contrast discrimination model.

According to the theory of comparative judgment developed by Thurstone $(1927 \mathrm{a}, 1927 \mathrm{~b})$, the chance for the observation of a difference between two stimuli is a function of the ratio between this difference and the uncertainty caused by the magnitude of the stimuli. For the here given model, it will be assumed that this 
uncertainty can be considered as a form of noise where the spatial frequency pattern of the reference signal is considered as the hypothetical noise source. This noise masks the observation of the difference between test signal and reference signal. The amount of this noise can be calculated with the aid of the expressions for nonwhite noise given in the previous chapter applied on a noise source consisting of a single spatial frequency pattern. The so obtained model will be compared with published data of contrast discrimination experiments.

\subsection{Evaluation of the psychometric function}

The psychometric function can give important information about the fundamental behaviour of the visual system at contrast discrimination. Fig. 7.1 shows the psychometric function for a contrast discrimination experiment by Foley \& Legge (1981). In this figure the detection probability $p$ for the modulation difference between test signal and reference signal is plotted as a function of the modulation $m$ of the test signal. The spatial frequency of the test pattern and the reference pattern was 2 cycles/deg and the modulation of the reference pattern was $0.23 \%$. The detection probability was calculated from the percentage of correct response in a 2 AFC experiment of the original data. The data points were the result of about 200 trials made at each stimulus (method of constant stimuli). The patterns were vertically oriented sinusoidal gratings displayed on a CRT monitor provided with P31 phos-

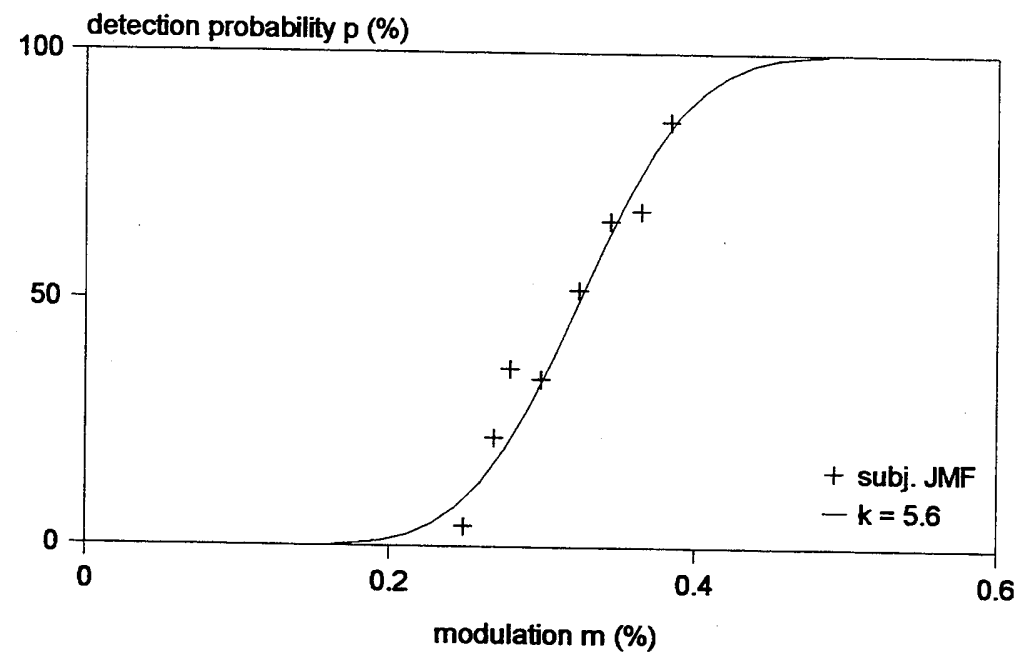

Figure 7.1: Psychometric function for a contrast discrimination experiment by Foley \& Legge (1981). Spatial frequency 2 cycles/deg. Modulation of the reference signal $0.23 \%$. The detection probability for the discrimination is plotted in the usual way as a function of the modulation $m$ of the test signal. For the curve through the data, $k=5.6$. 


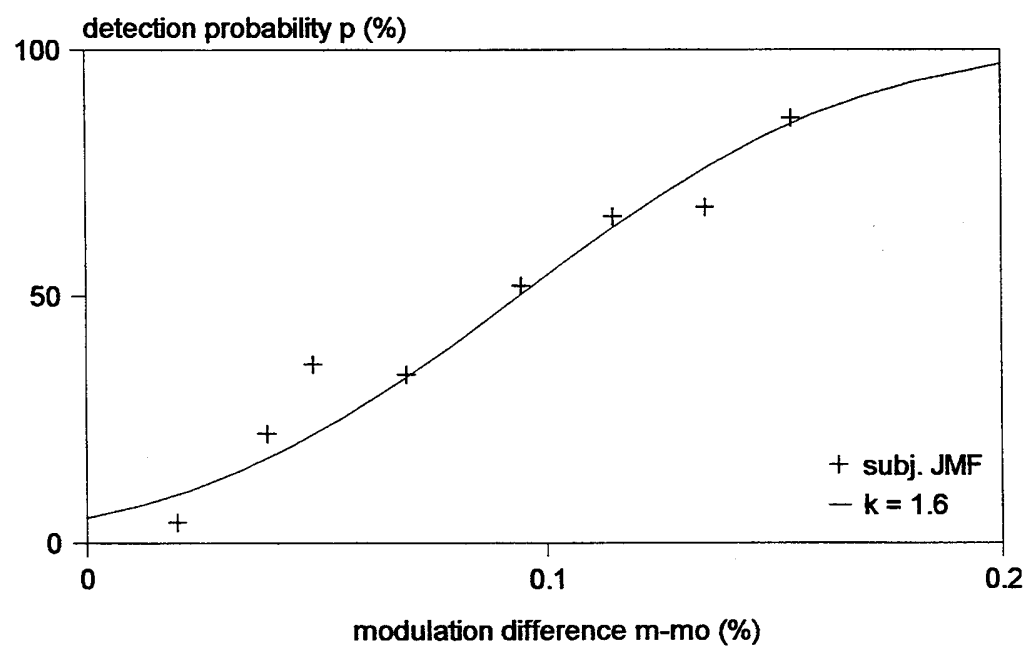

Figure 7.2: Psychometric function for the same data as Fig. 7.1, but plotted as a function of the modulation difference $m-m_{0}$ between test signal and reference signal. For the curve through the data, $k=1.6$.

phor. The luminance was $170 \mathrm{~cd} / \mathrm{m}^{2}$ and the field size was $6^{\circ} \times 6^{\circ}$. The test field was surrounded by a white surface area with a luminance that was approximately the same as the average luminance of the test field. Viewing was binocular with a natural pupil. The subject was JMF, the first author. The curve through the data has been calculated with the regression method mentioned in section 2.2 of Chapter 2 . The

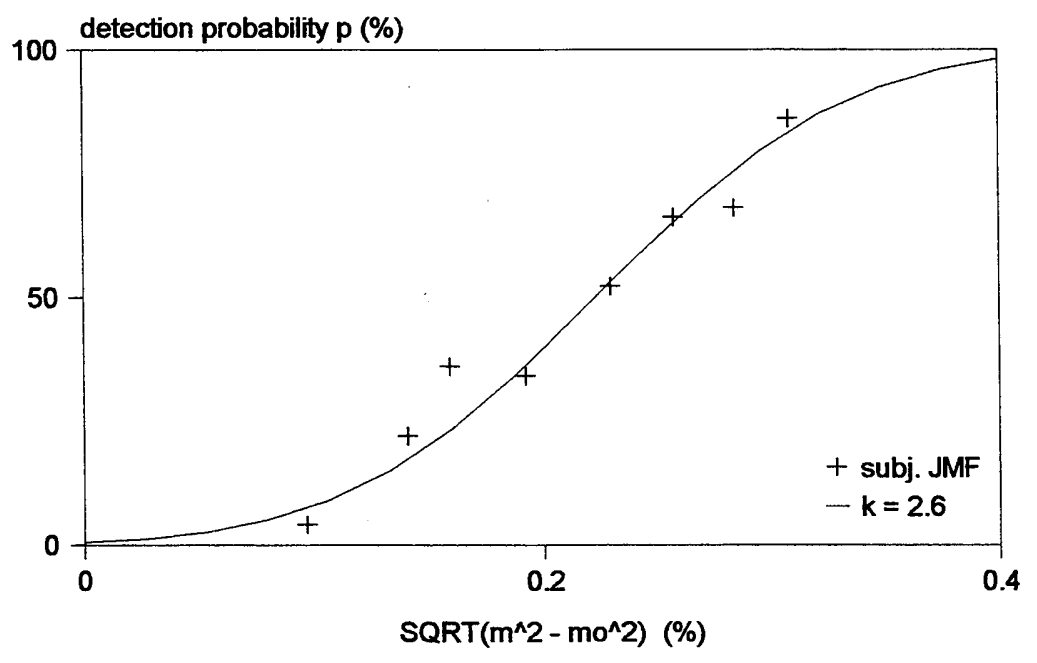

Figure 7.3: Psychometric function for the same data as Fig. 7.1, but plotted as a function of $\sqrt{ }\left(m^{2}-m_{0}{ }^{2}\right)$. For the curve through the data, $\mathrm{k}=2.6$. 
experiment was made under the same conditions and with the same subject as the detection experiment of Fig. 2.1 in Chapter 2. Compared with Fig. 2.1 the slope of the curve is much steeper. The $k$ value of this curve is 5.6 , which differs considerably from 3.0 that was found for the curve in Fig. 2.1. In Fig. 7.2 the same data are plotted as a function of the modulation difference $m-m_{0}$, where $m_{0}$ is the modulation of the reference signal. Here the slope of the curve through the data is much smaller and corresponds with a $k$ value 1.6. This value too differs considerably from that for the detection experiment of Fig. 2.1. The slope of the function obviously depends on the quantity used along the horizontal axis of the psychometric function. As it is assumed in this study that the $k$ value of the visual system must have a constant value of about 3, a different quantity for the horizontal axis of the psychometric function was sought, which would better represent the functional parameter of the contrast discrimination process. In Fig. 7.3 the same data are plotted as a function of $\sqrt{\left(m^{2}\right.}$ $\left.m_{0}{ }^{2}\right)$. Here the curve shows a slope corresponding with a $k$ value of 2.6 , which is close to the value 3.0 of Fig. 2.1.

As mentioned in the title of their paper, the measurements by Foley and Legge were made at a level of the reference signal close to the detection threshold. For higher levels of the reference signal, the same phenomena can be observed, but even in a more extreme way. Fig. 7.4 shows the psychometric function for a contrast discrimination experiment by Legge (1984a) at a spatial frequency of 0.5 cycles/deg with a modulation of the reference signal of $25 \%$. This modulation is about 50 times higher than the modulation threshold for detection. The detection probability was

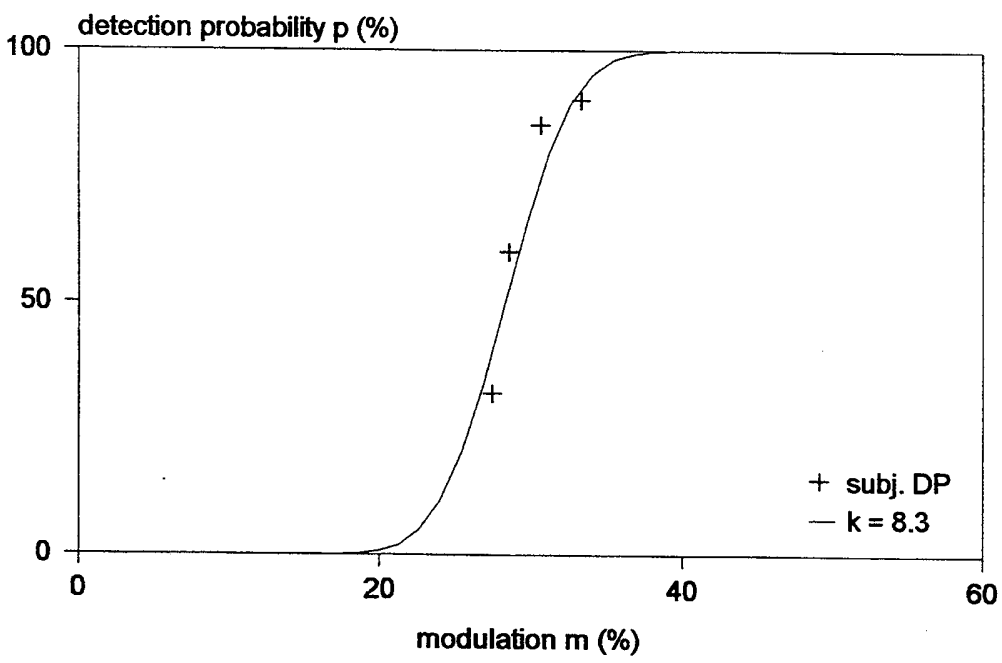

Figure 7.4: Psychometric function for a contrast discrimination experiment by Legge (1984a). Spatial frequency 0.5 cycles/deg. Modulation of the reference signal 25\%. The detection probability for the discrimination is plotted in the usual way as a function of the modulation $m$ of the test signal. For the curve through the data, $\mathrm{k}=8.3$. 


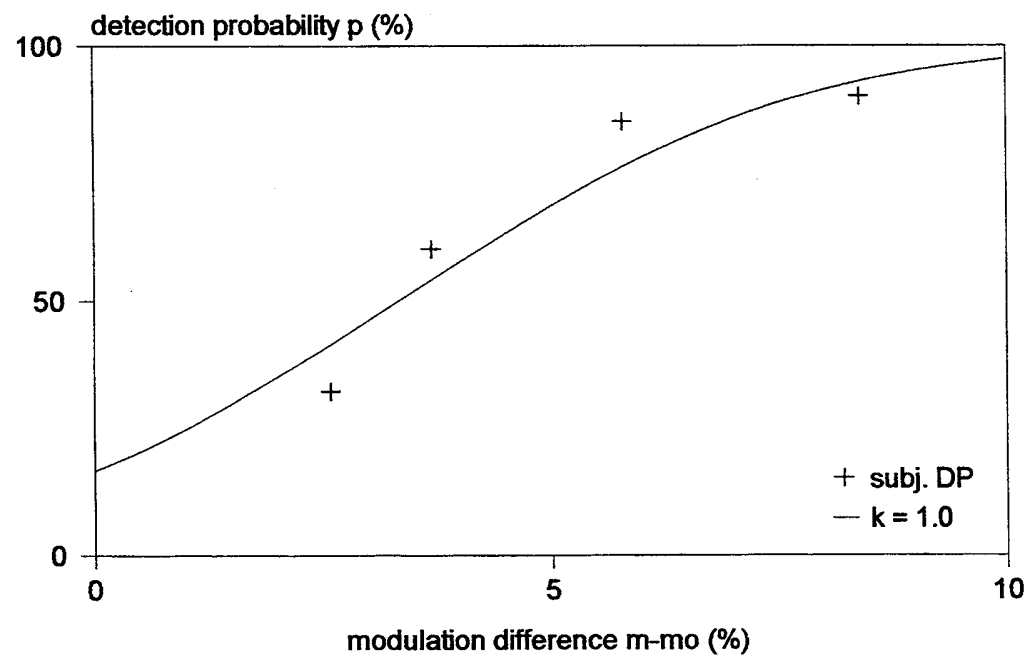

Figure 7.5: Psychometric function for the same data as Fig. 7.4, but plotted as a function of the modulation difference $m-m_{0}$ between test signal and reference signal. For the curve through the data, $k=1.0$.

calculated from the percentage of correct response in a 2AFC experiment of the original data. The data points were the result of about 240 trials made at each stimulus (method of constant stimuli). The patterns were vertically oriented sinusoidal gratings displayed on a CRT monitor provided with P31 phosphor. The luminance was $340 \mathrm{~cd} / \mathrm{m}^{2}$ and the field size was $11^{\circ} \times 6^{\circ}$. Viewing was binocular with a

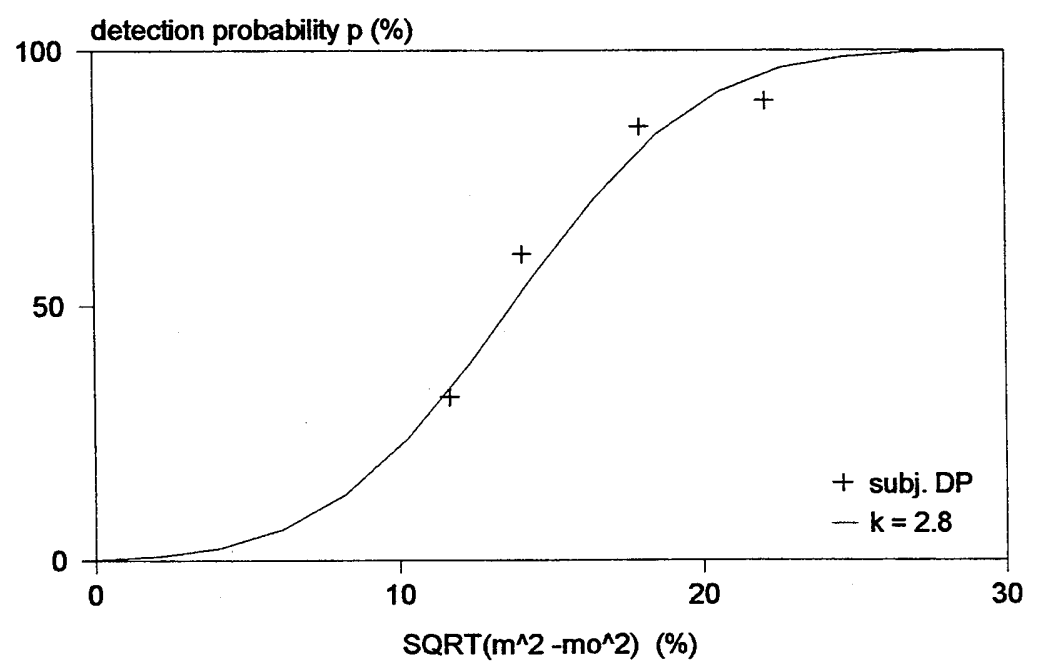

Figure 7.6: Psychometric function for the same data as Fig. 7.4, but plotted as a function of $\sqrt{ }\left(m^{2}-m_{0}{ }^{2}\right)$. For the curve through the data, $k=2.8$. 
natural pupil. The data are from one subject (DP) out of six observers, whom were all in their 20's. In Fig. 7.4 the psychometric function is plotted as a function of the modulation $m$ of the test signal. The slope of the curve corresponds with a $k$ value 8.3. In Fig. 7.5 the same data are plotted as a function of the modulation difference $m$ $m_{0}$, where $m_{0}$ is the modulation of the reference signal. The slope of the curve now corresponds with a $k$ value 1.0. In Fig 7.6 the same data are plotted as a function of $\sqrt{ }\left(m^{2}-m_{0}^{2}\right)$. Here the slope of the curve corresponds with a $k$ value 2.8 , which can be considered as the normal value.

From the given examples, it may be assumed that only if the psycho metric function is plotted as a function of $\sqrt{ }\left(m^{2}-m_{0}{ }^{2}\right)$ a normal value of $k$ is obtained. This means that for contrast discrimination, the quantity $\sqrt{ }\left(m^{2}-m_{0}\right)$ is in fact the functional parameter of the detection process.

\subsection{Evaluation of the contrast discrimination model}

The functional parameter $\sqrt{ }\left(m^{2}-m_{0}^{2}\right)$ for contrast discrimination given in the previous section can be written in the form $\sqrt{ }\left\{\left(m_{0}+\Delta m\right)^{2}-m_{0}{ }^{2}\right\}$ where $m_{0}$ is the modulation of the reference signal and $\Delta m$ is the modulation difference between the test signal and the reference signal. At the threshold $\Delta m=\Delta m_{\mathrm{t}}$, where $\Delta m_{\mathrm{t}}$ is the threshold of the modulation difference. This threshold appears to be a function of the modulation of the reference signal. It is assumed here that the influence of the reference signal on the threshold can be considered as a form of noise.

At detection, the modulation threshold in the presence of external noise is given by Eq. (2.50) of Chapter 2:

$$
m_{\mathrm{t}}^{\prime}=\sqrt{m_{\mathrm{t}}^{2}+k^{2} m_{\mathrm{n}}^{2}}
$$

where $m_{\mathrm{t}}$ is the modulation threshold without external noise, $m_{\mathrm{t}}^{\prime}$ is the increased modulation threshold with noise, and $m_{\mathrm{n}}$ is the average modulation of the noise wave components of the external noise. In analogy with this equation, the functional parameter for the threshold at contrast discrimination can be described by

$$
\sqrt{\left(m_{0}+\Delta m_{\mathrm{t}}\right)^{2}-m_{0}^{2}}=\sqrt{m_{\mathrm{t}}^{2}+k^{2} m_{\mathrm{n}}^{2}}
$$

where the left-hand side of the previous expression has been replaced by the functional parameter for contrast discrimination (Barten, 1995). This equation also holds when the modulation of the reference signal $m_{0}$ is zero. Then, $\Delta m_{\mathrm{t}}$ is equal to $m_{\mathrm{t}}{ }^{\prime}$, if there is external noise, or $\Delta m_{\mathrm{t}}$ is equal to $m_{\mathrm{t}}$, if there is no external noise. The equation can also be written in the form

$$
\Delta m_{\mathrm{t}}=\sqrt{m_{\mathrm{t}}^{2}+m_{0}^{2}+k^{2} m_{\mathrm{n}}^{2}}-m_{0}
$$


It is further assumed here that $m_{\mathrm{n}}$ is a function of $m_{0}$. The validity of this expression depends on the equation used for this function.

For this function, it is assumed that the reference signal can be considered as the noise source that influences the detection of a modulation difference between reference signal and test signal. The reference signal consists of a single sinusoidal spatial frequency component. It is assumed here that the bandwidth of this noise is very small and is equal to the just observable spatial frequency difference between two signals with different spatial frequency. For the calculation of the noise of this pattern, use can be made of the equations for nonwhite noise given in the previous chapter. For $m_{\mathrm{n}}$ follows from Eq. (6.1) after omitting $Y$ and $T$ because of the onedimensional static character of the noise:

$$
m_{\mathrm{n}}(u)=2 \sqrt{\frac{\Phi_{\mathrm{d}}(u)}{X}}
$$

The function $\Phi_{\mathrm{d}}(u)$ in this expression is given by Eq. (6.2). As the spatial frequency bandwidth of the noise source considered here is assumed to be small and the functions $\Psi\left(u, u_{n}\right)$ and $\Phi_{n}\left(u_{n}\right)$ are assumed to be constant within this frequency band (which means that $\Phi_{n}\left(u_{n}\right)$ is idealized as a rectangular distribution) Eq. (6.2) can be written in the form

$$
\Phi_{\mathrm{d}}(u)=\Psi\left(u, u_{\mathrm{n}}\right) \Phi_{\mathrm{n}}\left(u_{\mathrm{n}}\right) \frac{\Delta u_{\mathrm{n}}}{u}
$$

where $\Delta u$ is the bandwidth of the noise spectrum. For the situation considered here, $u_{n}$ is equal to $u$. For this situation, it follows from Eq. (6.4) that

$$
\Psi\left(u, u_{n}\right)=0.747
$$

It follows further from Eq. (2.43) for the spectral density of the reference signal that

$$
\Phi_{\mathrm{n}}=X\left(\frac{m_{0}}{2}\right)^{2}
$$

Insertion of these equations in Eq.(7.4) gives

$$
\Phi_{\mathrm{d}}(u)=0.747 X\left(\frac{m_{0}}{2}\right)^{2} \frac{\Delta u}{u}
$$

and insertion of this equation in Eq. (7.3) gives

$$
m_{\mathrm{n}}(u)=\sqrt{0.747 \frac{\Delta u}{u}} m_{0}
$$

The quantity $\Delta u$ in this expression is the bandwidth of the noise spectrum. This bandwidth was assumed to be equal to the just observable spatial frequency difference between two signals. Campbell et al. (1970) measured this difference for a large range of spatial frequencies extending from 0.6 to $30 \mathrm{cycles} / \mathrm{deg}$. They found a value of about $5-6 \%$ for $\Delta u / u$ independent of spatial frequency and field size. Similar results 
were found by Burbeck \& Regan (1983) and by Regan (1985) who varied also some additional conditions. This means that it may be assumed that $\Delta u / u \approx 0.055$. Insertion of this value in Eq. (7.8) gives

$$
m_{\mathrm{n}}=0.2 m_{0}
$$

and insertion of this expression in Eq. (7.2) gives

$$
\Delta m_{\mathrm{t}}=\sqrt{m_{\mathrm{t}}^{2}+\left(1+0.04 k^{2}\right) m_{0}^{2}}-m_{0}
$$

Carlson \& Cohen (1980) already suggested a similar equation. However, their model is based on the assumption that the detection process for the modulation difference is determined by a constant fraction of the reference modulation. This fraction depends on the spatial frequency and the average luminance and has to be found experimentally. According to the model given here, $\Delta m_{\mathrm{t}}$ is independent of spatial frequency and luminance, and depends only on $k$.

If $\Delta m_{\mathrm{t}}$ given by Eq. (7.10) is plotted as a function of the modulation of the reference signal, a dipper-shape curve is obtained, which is well known from experiments. See the measured data in Figs. (7.7) through (7.9). However, at high reference signal modulations, the calculated curve shows a linear increase with the modulation of the reference signal, whereas experimental data show a slight downward bend at these modulations. This bend is caused by the nonlinear behaviour of the visual system at high modulation levels. Wilson (1980) proposed to take the nonlinearity into account by using a transducer function. This method is, however, rather complicated. Here a simpler method will be followed. For this purpose Eq. (7.10) is first written in a form similar to Eq. (7.1):

$$
\sqrt{\left(m_{0}+\Delta m_{\mathrm{t}}\right)^{2}-m_{0}^{2}}=\sqrt{m_{\mathrm{t}}^{2}+0.04 k^{2} m_{0}^{2}}
$$

In this equation, the left-hand side represents the functional parameter of the contrast discrimination process. The right-hand side can now be divided by a factor $\sqrt{ }\left(1+c m_{0}\right)$ where $c$ is a constant describing the nonlinearity. This modifies the equation into

From this equation follows that

$$
\sqrt{\left(m_{0}+\Delta m_{\mathrm{t}}\right)^{2}-m_{0}{ }^{2}}=\sqrt{\frac{m_{\mathrm{t}}^{2}+0.04 k^{2} m_{0}^{2}}{1+c m_{0}}}
$$

$$
\Delta m_{\mathrm{t}}=\sqrt{\frac{m_{\mathrm{t}}^{2}+0.04 k^{2} m_{0}^{2}}{1+c m_{0}}+m_{0}{ }^{2}}-m_{0}
$$

It may be assumed that the nonlinearity constant $c$ is determined by the ratio between the modulation $m_{0}$ of the reference signal and the average modulation of the internal noise in the visual system. This average modulation is equal to $m_{\mathrm{t}} / k$. This means that $c$ is some numerical factor divided by $m_{\mathrm{t}} / k$. From a comparison with the measured data that will be given in the following sections, it appears that this factor 
is about 0.004 . This means that

$$
c \approx 0.004 \mathrm{k} / \mathrm{m}_{\mathrm{t}}
$$

With this value of $c$, the equation for the contrast discrimination threshold finally becomes

$$
\Delta m_{\mathrm{t}}=\sqrt{\frac{m_{\mathrm{t}}^{2}+0.04 k^{2} m_{0}^{2}}{1+0.004 k m_{0} / m_{\mathrm{t}}}+m_{0}{ }^{2}}-m_{0}
$$

In the following section, published data of contrast discrimination measurements will be compared with the results obtained with this equation.

\subsection{Comparison with contrast discrimination measurements}

Fig. 7.7 shows contrast discrimination measurements by Nachmias \& Sansbury (1974) for a vertically oriented sinusoidal grating pattern with a spatial frequency of 3 cycles/deg. The pattern was displayed on a CRT monitor provided with P31 phosphor. The field size of the stimulus was $2.2^{\circ} \times 3.2^{\circ}$ and was surrounded by an area with the same luminance and colour as the stimulus. The luminance was not mentioned but was probably about $100 \mathrm{~cd} / \mathrm{m}^{2}$. The threshold was determined with a temporal 2AFC method where the threshold corresponded with $79.4 \%$ correct

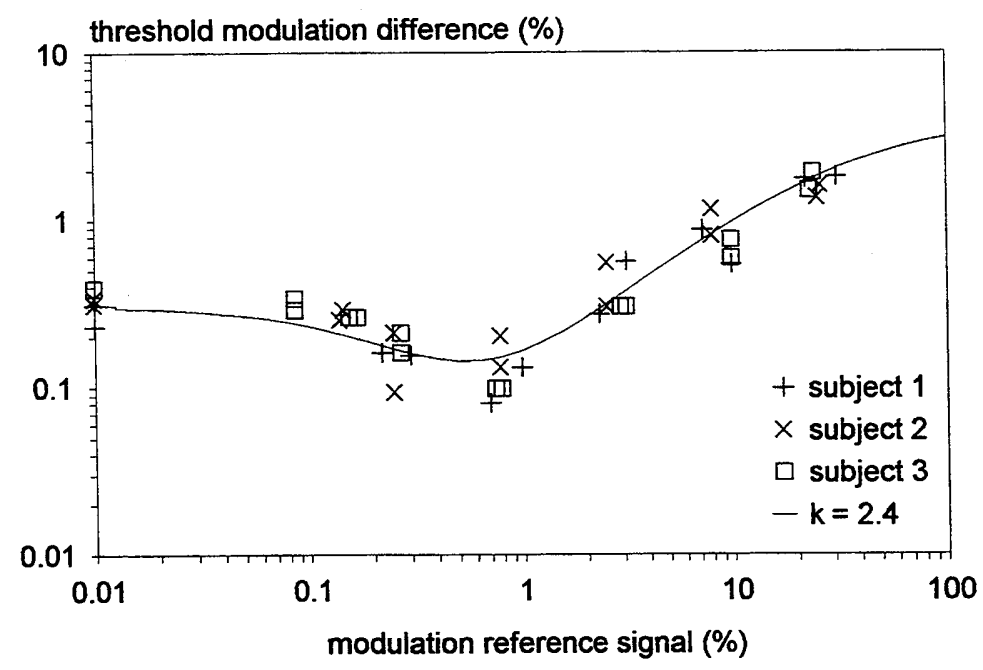

Figure 7.7: Contrast discrimination measurements by Nachmias \& Sansbury (1974) for a spatial frequency of 3 cycles/deg and a field size of $2.2^{\circ} \times 3.2^{\circ}$. The curve through the data has been calculated with Eq. (7.15) with $\mathrm{k}=2.4$. 
response. Three observers took part in the investigation. The curve through the data has been calculated with Eq. (7.15) using for $k$ a value of 2.4 after correcting the difference between $79.4 \%$ and $75 \%$ correct response with the aid of Eq. (2.14). The calculated curve agrees well with the average results of the data.

Figs. 7.8 and 7.9 show similar measurements by Legge \& Foley (1980) for a vertically oriented sinusoidal grating pattern with a spatial frequency of 2 cycles/deg. The pattern was displayed on a CRT monitor provided with P31 phosphor with a luminance of $200 \mathrm{~cd} / \mathrm{m}^{2}$. For the measurements given in Fig. 7.8, the field size was $0.75^{\circ} \times 6^{\circ}$, and for the measurements given in Fig. 7.9, the field size was $6^{\circ} \times 6^{\circ}$. The test field was surrounded by a white cardboard at the same luminance and viewed from a distance of $1.14 \mathrm{~m}$. Viewing was binocular with a natural pupil. The threshold was determined with a temporal 2AFC method where the threshold corresponded with $79 \%$ correct response. Three observers took part in the investigation. The reported data points were the geometric means of the measurement data. The curves through the data have been calculated with Eq. (7.15) using for $k$ a value of 3.0 in Fig. 7.8 and 4.0 in Fig. 7.9 after correcting the difference between $79 \%$ and $75 \%$ correct response with the aid of Eq. (2.14). Both figures show a good agreement between measurements and calculations.

For contrast discrimination experiments where the measurements are made with monocular vision, instead of binocular vision, the same expression is valid. The only difference is that for $m_{\mathrm{t}}$ the modulation threshold for monocular viewing must be used, which is a factor $\sqrt{2}$ higher. The difference between monocular and binocular

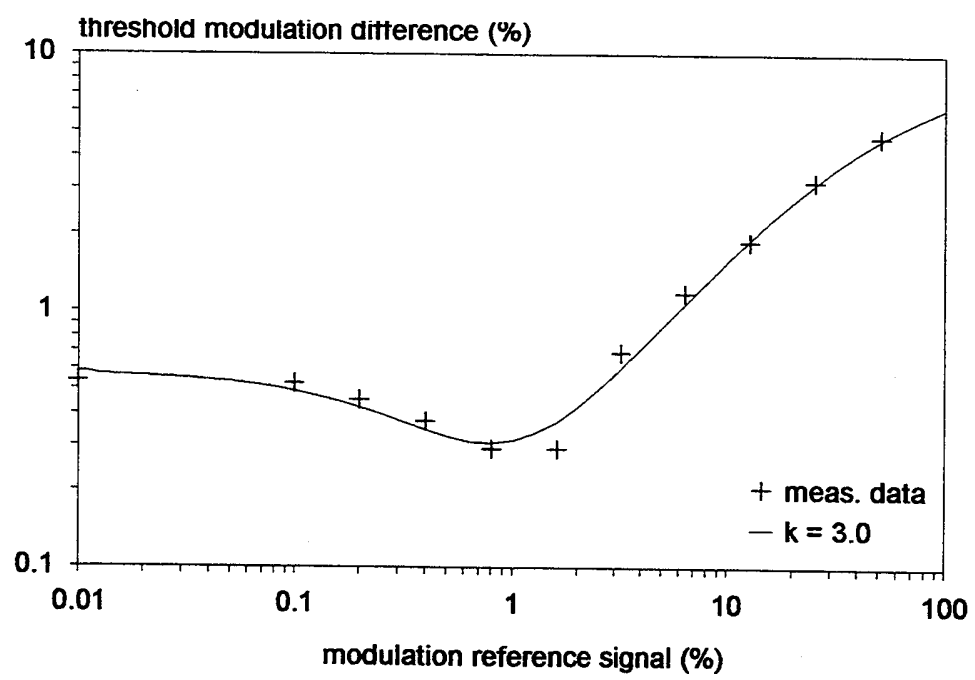

Figure 7.8: Contrast discrimination measurements by Legge \& Foley (1980) for a spatial frequency of $2 \mathrm{cycles} / \mathrm{deg}$ and a field size of $0.75^{\circ} \times 6^{\circ}$. Luminance $200 \mathrm{~cd} / \mathrm{m}^{2}$. Binocular viewing. The curve through the data has been calculated with Eq. (7.15) with $\mathrm{k}=3.0$. 


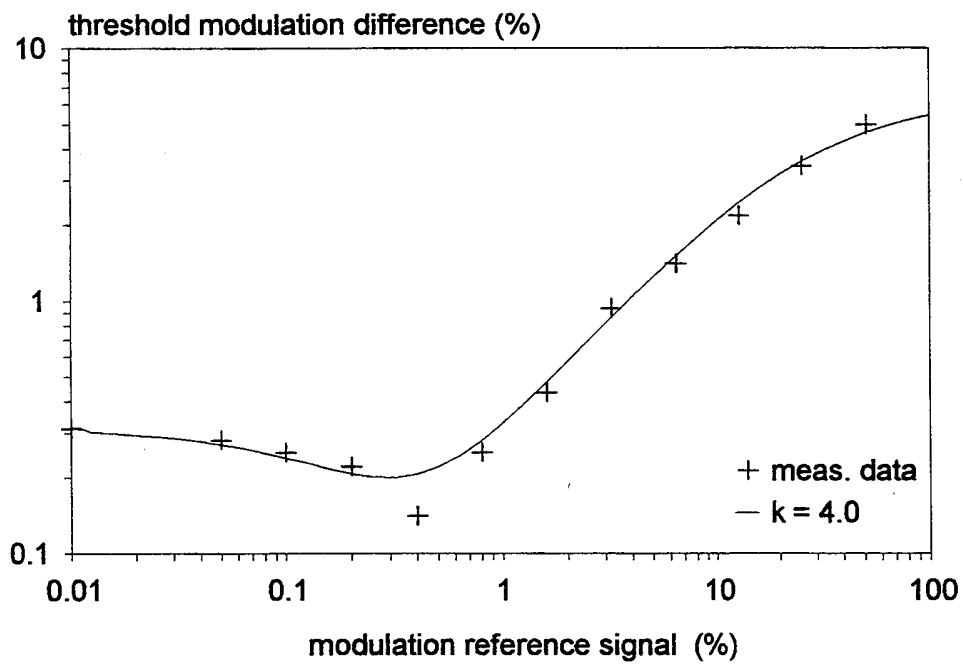

Figure 7.9: Same measurements as in Fig. 7.8 but for a field size of $6^{\circ} \times 6^{\circ}$. The continuous curve has been calculated with Eq. $(7.15)$ with $\mathrm{k}=4.0$.

vision only occurs at low modulations of the reference signal. At higher modulations, $m_{\mathrm{t}}$ is small compared with $m_{0}$ so that the effect of $m_{\mathrm{t}}$ disappears. See Eq. (7.15). This is illustrated in Fig. 7.10 where measurements by Legge (1984b) are shown made with monocular and binocular vision. The measurements were made in the same

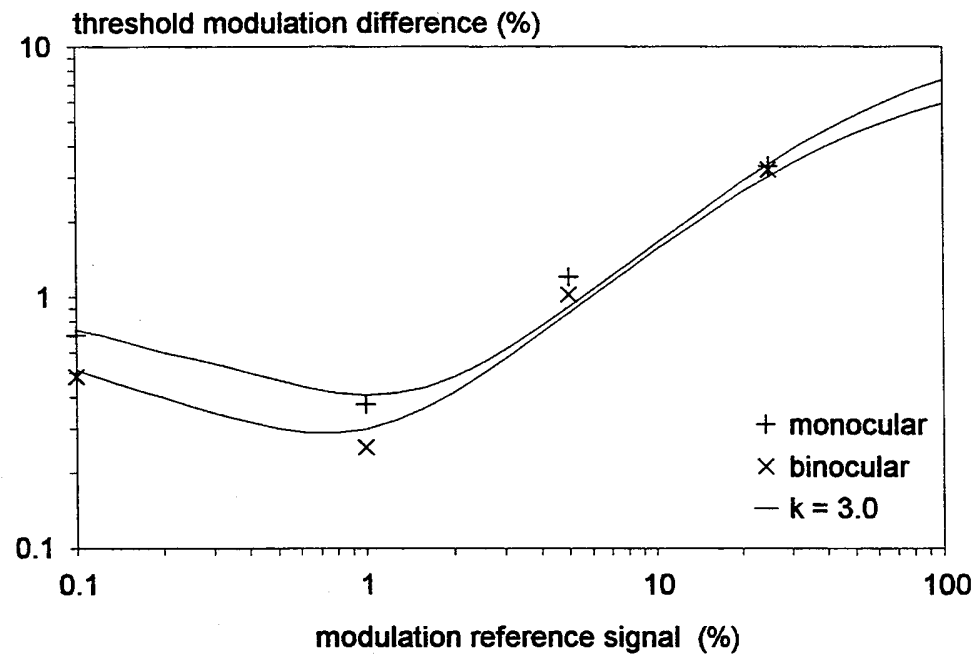

Figure 7.10: Monocular and binocular contrast discrimination measurements made by Legge $(1984 \mathrm{~b})$ for a spatial frequency of $0.5 \mathrm{cycles} / \mathrm{deg}$ and a field size of $11^{\circ} \times 6^{\circ}$. Luminance $340 \mathrm{~cd} / \mathrm{m}^{2}$. The curve through the data has been calculated with Eq. (7.15) with $\mathrm{k}=3.0$. 
investigation as the measurements of the psychometric function shown in Figs. 7.4 through 7.6. Although the number of measurement data is small, they clearly show the expected difference with a factor $\sqrt{2}$ at a low modulation of the reference signal.

If external noise is present, Eq. (7.15) has to be extended in the following way:

$$
\Delta m_{\mathrm{t}}=\sqrt{\frac{m_{\mathrm{t}}^{2}+k^{2} m_{\mathrm{n}}^{2}+0.04 k^{2} m_{0}^{2}}{1+0.004 k m_{0} / m_{\mathrm{t}}}+m_{0}^{2}}-m_{0}
$$

where $m_{n}$ is the average modulation of the external noise, which can be calculated from the noise data with Eqs. (2.41) and (2.43) given in Chapter 2. Fig. 7.11 shows the results of contrast discrimination measurements by Pelli (1985) with and without two-dimensional dynamic noise. He used a vertically oriented sinusoidal grating pattern with a spatial frequency of 4 cycles/deg and a luminance of $300 \mathrm{~cd} / \mathrm{m}^{2}$. The stimulus was vignetted by a Gaussian envelope to a field size of $4^{\circ} \times 4^{\circ}$ and an exposure time of $70 \mathrm{msec}$ in time (1/e value). Two noise levels were used, with a spectral density of $0.9 \times 10^{-6} \mathrm{sec} \mathrm{deg}^{2}$ and $9 \times 10^{-6} \mathrm{sec} \mathrm{deg}^{2}$, respectively. However, these values had to be corrected with a factor $2^{3}$ due to a different definition of spectral noise density. The measurements at zero modulation of the reference signal have already been mentioned in Table 2.1 of Chapter 2. The threshold was determined with a temporal 2 AFC method where the threshold corresponded with $82 \%$ correct response. The curves through the data have been calculated with Eq. (7.16)

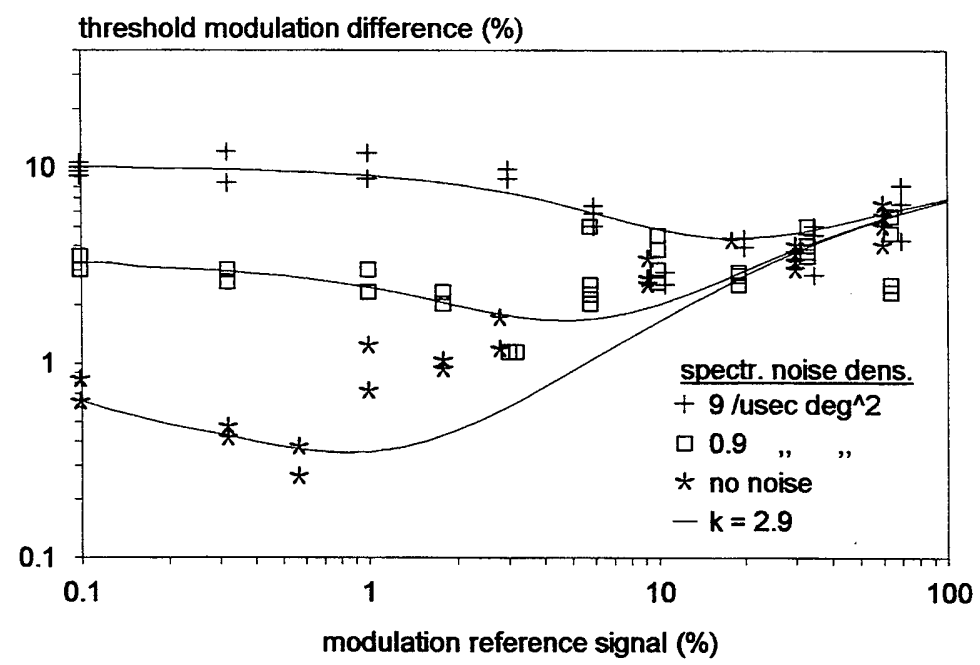

Figure 7.11: Contrast discrimination measurements by Pelli (1985) with and without two-dimensional dynamic noise for a spatial frequency 4 cycles/deg and a field size of $4^{\circ} \times 4^{\circ}$. Luminance $300 \mathrm{~cd} / \mathrm{m}^{2}$. The curves through the data have been calculated with Eq.(7.16) with $\mathrm{k}=2.9$. 
using for $k$ a value of 2.9 after correcting the difference between $82 \%$ and $75 \%$ correct response with the aid of Eq. (2.14). This value has already been mentioned in Table 2.1 of Chapter 2. For the noise-free situation, the agreement between measurements and calculations is less good than in the preceding figures. However, for the situation with noise the general behaviour is well described.

\subsection{Generalized contrast discrimination model}

The threshold of the modulation difference given by Eq. (7.15) depends apart from $m_{0}$ and $k$ also on $m_{\mathrm{t}}$. Contrast discrimination curves where $\Delta m_{\mathrm{t}}$ is plotted as a function of $m_{0}$ will therefore be different for different spatial frequencies. Legge (1979) noticed that contrast discrimination curves for different spatial frequencies coincide if they are plotted in a normalized way by dividing the modulation along both axes by the modulation threshold for detection. This is confirmed by the contrast discrimination model given here. If a relative threshold is introduced for the modulation difference defined by $\Delta m_{\text {trel }}=\Delta m_{\mathrm{t}} / m_{\mathrm{t}}$ and a relative modulation is introduced for the reference signal defined by $m_{\text {rel }}=m_{\delta} / m_{\mathrm{t}}$, Eq. (715) can be transformed in the following generalized expression:

$$
\Delta m_{\text {trel }}=\sqrt{\frac{1+0.04 k^{2} m_{\mathrm{rel}}^{2}}{1+0.004 k m_{\mathrm{rel}}}+m_{\mathrm{rel}}{ }^{2}}-m_{\mathrm{rel}}
$$

In this equation $\Delta m_{\text {trel }}$ depends apart from $m_{\text {rel }}$ only on $k$. Therefore, this generalized expression for contrast discrimination is independent from spatial frequency, luminance and field size.

Fig. 7.12 shows a plot of the generalized equation with contrast discrimination measurements made by Legge (1979) for four different spatial frequencies: $0.25,1$, 4 , and 16 cycles/deg. The grating patterns were displayed on a CRT monitor provided with P31 phosphor with a luminance of $200 \mathrm{~cd} / \mathrm{m}^{2}$. Viewing was monocular with a natural pupil. A split-screen arrangement was used in which the reference signal and the test signal were presented on the left half of the display and a uniform field of the same luminance on the right half of the display. A vertical cardboard divider stood between the center of the display and the observer's eyes so that the left and right halves of the screen were visible only to the left and right eyes, respectively. Both half-fields were $13 \mathrm{~cm} \times 20 \mathrm{~cm}$. For the gratings of 0.25 and 1 cycles $/ \mathrm{deg}$, the viewing distance was $0.57 \mathrm{~m}$ and the half-fields subtended $13^{\circ} \times 20^{\circ}$. For the gratings of 4 and $16 \mathrm{cycles} / \mathrm{deg}$ the viewing distance was $2.28 \mathrm{~m}$ and the half-fields subtended $3.25^{\circ} \times 5^{\circ}$. The threshold was determined with a temporal 2 AFC method where the threshold corresponded to $79 \%$ correct response. The observers were CF, a male in his early twenties and JG, a female with 19-years of age. The reported data points were the geometric means of 12 threshold estimates made by each of both observers. For 
rel. threshold modulation difference

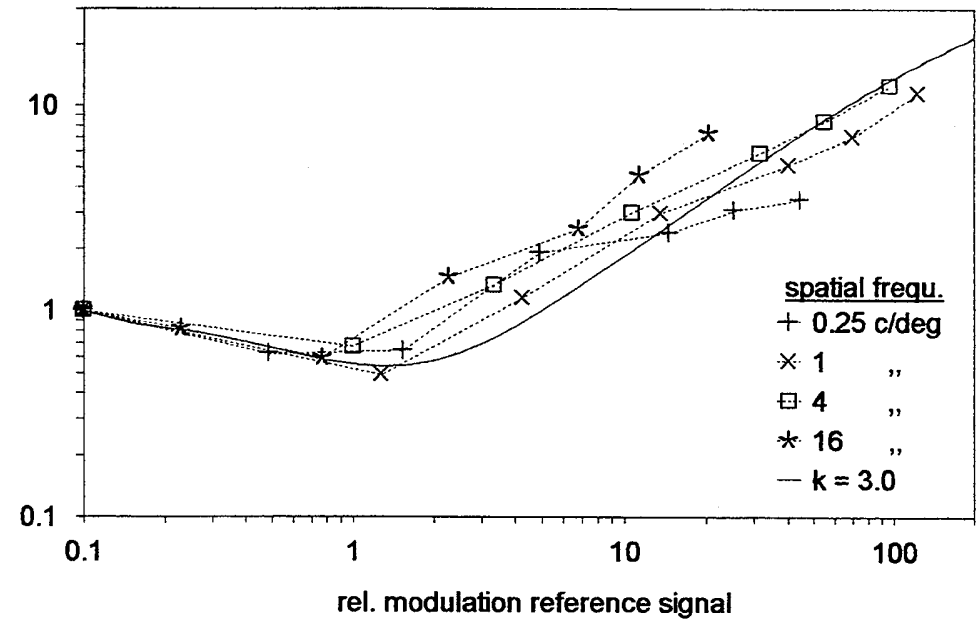

Figure 7.12: Contrast discrimination measurements by Legge (1979) for different spatial frequencies plotted with normalized coordinates. Luminance $200 \mathrm{~cd} / \mathrm{m}^{2}$. Monocular viewing with a natural pupil. The generalized curve through the data has been calculated with Eq. (7.17) with $\mathrm{k}=3.1$.

the calculation of the generalized curve in Fig. $7.12 \mathrm{a} k$ value of 3.0 was used after correcting the difference between $79 \%$ and $75 \%$ correct response with the aid of Eq. (2.14). Although the data points show a rather large spread the general agreement between measurements and calculations is reasonable.

In this investigation by Legge also an experiment was made with dichoptic viewing. In this experiment the reference signal was presented on the left half of the screen, whereas the test signal pattern was presented on the right half of the screen. This presentation was followed or preceded by a presentation where both halves of the screen contained only the reference signal. The observer had to identify which of the two intervals contained the test signal. Binocular fusion was aided by black fixation dots at the centres of the half-fields and by using prisms in front of the eyes.

The results of this dichoptic viewing experiment can probably be explained by modifying Eq. (7.1) that describes the functional parameter for contrast discrimination. This equation can be modified as follows:

$$
\sqrt{1 / 2\left\{\left(m_{0}+\Delta m_{\mathrm{t}}\right)^{2}-m_{0}{ }^{2}\right\}}=\sqrt{m_{\mathrm{t}}^{2}+2 k^{2} m_{\mathrm{n}}^{2}(u)}
$$

where a factor $1 / 2$ has been added at the left-hand side and a factor two has been added at the right-hand side. The factor $1 / 2$ at the left-hand side has been added because the test signal is presented to only one eye, so that only half of the difference is effective for the detection. The factor two in front of the second term at the right- 
hand side has been added because only one of the four fields contains an increase of the modulation of the reference signal, whereas otherwise one of the two fields had contained an increase of modulation. Although this last argument seems somewhat artificial, it appears to be justified by a comparison with the measurement data. Multiplication of both sides of the equation with $\sqrt{2}$ gives

$$
\sqrt{\left(m_{0}+\Delta m_{\mathrm{t}}\right)^{2}-m_{0}^{2}}=\sqrt{2 m_{\mathrm{t}}^{2}+4 k^{2} m_{\mathrm{n}}^{2}(u)}
$$

In this expression $m_{\mathrm{t}}$ is the detection threshold for binocular vision. If one replaces this threshold for numerical reasons by the detection threshold for monocular vision, which is a factor $\sqrt{2}$ higher, one obtains

$$
\sqrt{\left(m_{0}+\Delta m_{\mathrm{t}}\right)^{2}-m_{0}^{2}}=\sqrt{m_{\mathrm{t}}^{2}+4 k^{2} m_{\mathrm{n}}^{2}(u)}
$$

In this equation the term with $m_{\mathrm{n}}(u)^{2}$ is multiplied with a factor 4 compared with the situation in Eq. (7.1). If this factor is introduced in Eq. (7.17), one obtains as generalized expression for the dichoptic viewing conditions discussed here

$$
\Delta m_{\mathrm{trel}}=\sqrt{\frac{1+4 \times 0.04 k^{2} m_{\mathrm{rel}}^{2}}{1+0.004 k m_{\mathrm{rel}}}+m_{\mathrm{rel}}^{2}}-m_{\mathrm{rel}}
$$

Fig. 7.13 shows a plot of this equation with the results of the dichoptic viewing experiment by Legge. For $k$ a value 3.3 was used after correcting the difference between $79 \%$ and $75 \%$ correct response with the aid of Eq. (2.14). Contrary to Fig. 7.12 , the measurement data show here a small spread and a very good agreement with the calculated curve.

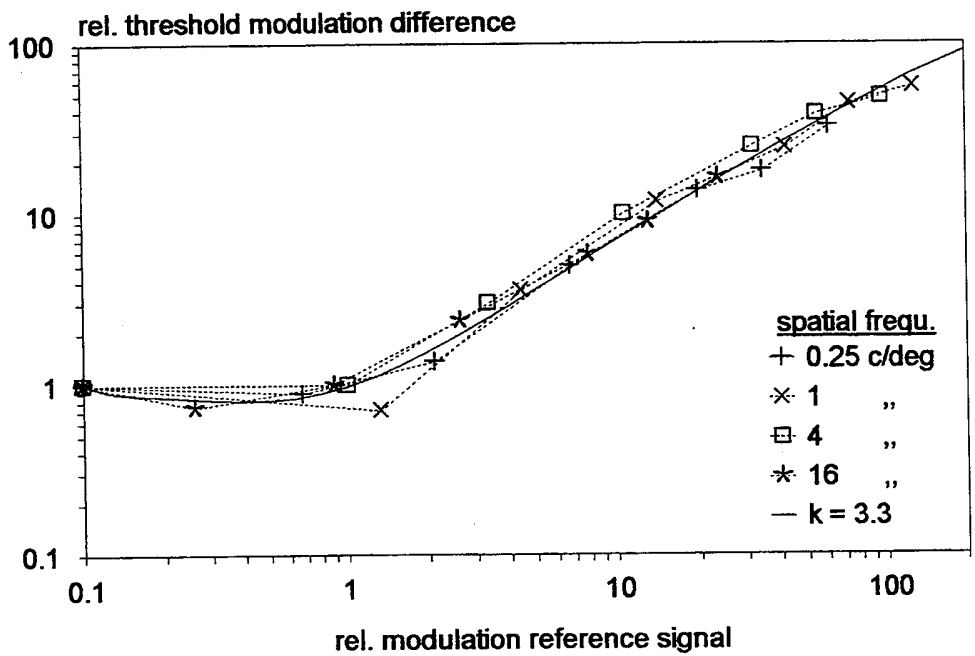

Figure 7.13: Same measurements as in Fig. 7.12 but for dichoptic viewing conditions. The generalized curve has been calculated with Eq. (7.21) with $\mathrm{k}=3.3$. 


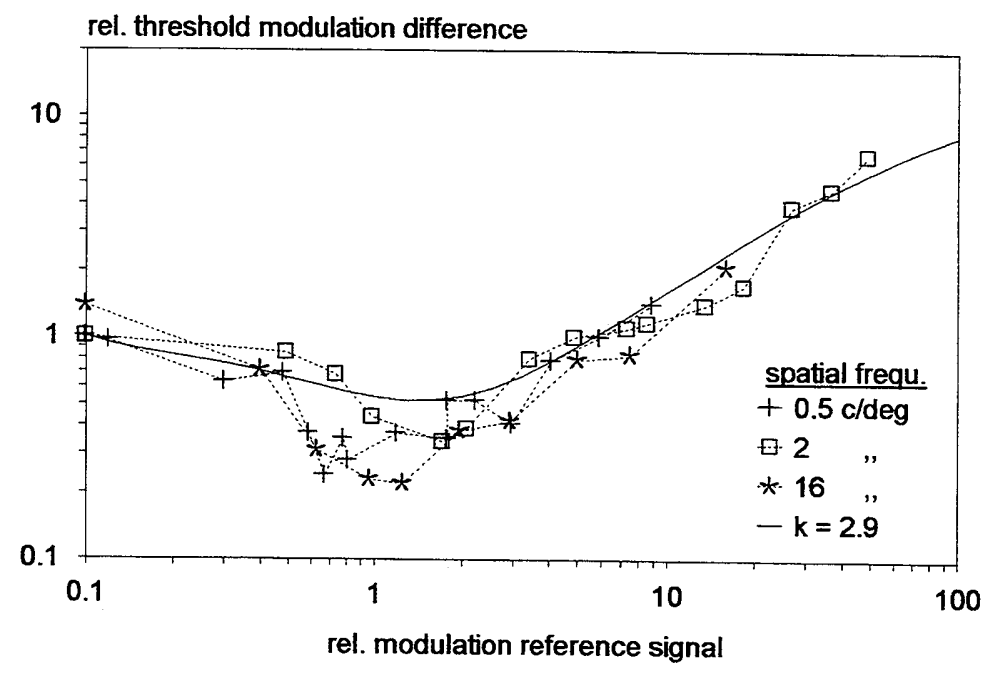

Figure 7.14: Contrast discrimination measurements made by Bradley \& Ohzawa (1986) for different spatial frequencies plotted with normalized coordinates. Luminance 250 $\mathrm{cd} / \mathrm{m}^{2}$. Monocular viewing with an artificial pupil of $2.5 \mathrm{~mm}$. The solid curve has been calculated with Eq.(7.17) with $\mathrm{k}=2.9$.

Fig. 7.14 shows measurements by Bradley \& Ohzawa (1986) for three different spatial frequencies: 0.5 cycles/deg, 2 cycles/deg and 16 cycles/deg, plotted with normalized coordinates. The stimuli were displayed on a CRT monitor with a luminance of $250 \mathrm{~cd} / \mathrm{m}^{2}$ and viewed through a mask with a circular aperture of $20 \mathrm{~cm}$. The mask had a uniform luminance of approximately $20 \mathrm{~cd} / \mathrm{m}^{2}$. The spatial frequency was varied by varying the number of cycles on the screen and by varying the viewing distance. The modulation threshold was determined with a $2 \mathrm{AFC}$ method where the threshold corresponded with $79 \%$ correct response. Viewing was monocular with an artificial pupil with a diameter of $2.5 \mathrm{~mm}$. Two observers participated in the experiments. The data are the average results of these two observers. The solid curve has been calculated with Eq. (7.17) using a $k$ value of 2.9 after correcting the difference between $79 \%$ and $75 \%$ correct response with the aid of Eq. (2.14). Similarly to the measurements by Legge shown in Fig. 7.12, the measurements show some spread around the generalized curve, but they do not show a systematic dependence on spatial frequency. Although the general agreement between measurements and calculations is reasonable, the measurements show a tendency to a deeper minimum. The average deviation of the measurements from the calculated curve is opposite to the deviation of the measurements shown in Fig. 7.12. 


\subsection{Summary and conclusions}

An analysis of the psychometric function for measurements of contrast discrimination given in this chapter has shown that the square root of the difference in squared modulation of the test signal and the reference signal is the functional parameter in the contrast discrimination process. With the aid of this parameter a model has been developed for the contrast discrimination between sinusoidal luminance patterns. In this model, the effect of the reference signal on the just observable modulation difference between the test signal and the reference signal has been described as an effect of noise, where the reference signal is considered as noise source. The bandwidth of this noise has been assumed to be equal to the just noticeable spatial frequency difference between two signals. With the aid of the equations for nonwhite noise given in the previous chapter, the amount of noise could be expressed in the modulation of the reference signal. Furthermore, an adaptation has been made for the nonlinearity of the eye at high modulation levels. In this way a contrast discrimination model has been obtained that appeared to be in good agreement with published measurements. Also the effect of noise on contrast discrimination could be explained, and the effects of binocular, monocular and dichoptic viewing.

By dividing the threshold of the modulation difference and the modulation of the reference signal by the modulation threshold for detection, a generalized expression for contrast discrimination has been obtained, which is independent from spatial frequency, luminance and field size. The existence of such a relation was already experimentally found by Legge (1979) and Bradley \& Ohzawa (1986), but remained until now without explanation.

\section{References}

Barten, P.G.J. (1995). Simple model for spatial frequency masking and contrast discrimination. Human Vision, Visual processing, and Digital Display VI, Proc. SPIE, $2411,142-158$.

Bradley, A. \& Ohzawa, I. (1986). A comparison of contrast detection and discrimination. Vision Research, 26, 991-997.

Burbeck, C.A. \& Regan, D. (1983). Independence of orientation and size in spatial discriminations. Journal of the Optical Society of America, 73, 1691-1694.

Campbell F.W., Nachmias J., and Jukes J. (1970). Spatial-frequency discrimination in human vision. Journal of the Optical Society of America, 60, 555-559.

Carlson, C.R. \& Cohen, RW. (1980). A simple psychophysical model for predicting 
the visibility of displayed information. Proceedings of the SID, 21, 229-246.

Foley, J.M. \& Legge, G.E. (1981). Contrast detection and near-threshold discrimination in human vision. Vision Research, 21, 1041-1053.

Legge, G.E. (1979). Spatial frequency masking in human vision: binocular interac tions. Journal of the Optical Society of America, 69, 838-847.

Legge, G.E. (1984a). Binocular contrast summation-I. Detection and discrimination. Vision Research, 24, 373-383.

Legge, G.E. (1984b). Binocular contrast summation-II. Quadratic summation. Vision Research, 24, 385-394.

Legge, G.E. \& Foley, J.M. (1980). Contrast masking in human vision. Journal of the Optical Society of America, 70, 1458-1471.

Nachmias, J. \& Sansbury, R.V. (1974). Grating contrast: discrimination may be better than detection. Vision Research, 14, 1039-1042.

Pelli, D.G. (1985). Uncertainty explains many aspects of visual contrast detection and discrimination. Journal of the Optical Society of America A, 2, 1508-1532.

Regan, D. (1985). Storage of spatial-frequency information and spatial-frequency discrimination. Journal of the Optical Society of America A, 2, 619-621.

Thurstone, L.L. (1927a). Psychophysical analysis. American Journal of Psychology, 38, 368-389.

Thurstone, L.L. (1927b). A law of comparative judgment. Psychological Review, 34, 273-286.

Wilson, H.R. (1980). A transducer function for threshold and supathreshold human vision. Biological Cybernetics, 38, 171-178. 


\section{Chapter 8}

\section{Image quality measure}

\subsection{Introduction}

The contrast discrimination model given in the previous chapter will be used in this chapter for the derivation of a measure for the perceived quality of an image. The quality of an image has always been an important aspect in the design of image forming systems. These systems can be cameras and printers for photographic systems, film projectors for movie systems, display units for television systems, imaging systems for medical and scientific applications, etc. For the design and judgment of these systems a quantified measure of the image quality is needed. However, the perceived quality of an image depends not only on the physical parameters of the system, like resolution and contrast, but also on the impression of the image received by the eye of the observer. Therefore, the judgment by a number of observers is often used as a quantitative measure for the obtained image quality. As this method is quite laborious and still rather subjective, investigators have searched for an objective measure for image quality in the form of a mathematical expression that contains a weighted combination of the physical parameters of the image and the psychophysical parameters of the human visual system. Such an expression is called an image quality metric. As physical parameter, the MTF of the imaging system is generally used, and as psychophysical parameter, the contrast sensitivity function of the human eye. The metrics differ from each other in the way these parameters are combined and in the way the image quality contributions of the different spatial frequency components are taken into account. In this chapter a survey will be given of the most important of these metrics.

Although the development of these metrics has contributed much to a better understanding of the effect of various parameters on image quality, they usually lack a good correlation with subjective image quality. This is partly caused by the fact that in most metrics, it is assumed that the perceived image quality is linearly related with the MTF of the imaging system and therefore with the modulation of the spatial frequency components of the image. A linear relation may be valid for modulations 
at threshold level, but the largest part of an image consists of modulations at suprathreshold level. At suprathreshold level the nonlinear behaviour of the visual system has to be taken into account. Therefore, first a model will be given for this nonlinear behaviour. This model will be based on the fact that perceived image quality appears to be linearly related with the number of discriminable levels of the modulations that occur in the image. For this model, use will be made of the generalized equation for contrast discrimination given in the previous section. This model appears also to give a good description of the subjectively perceived contrast of sinusoidal luminance patterns as a function of modulation. It will be used as basis for the here given image quality metric where the nonlinear behaviour of the eye is taken into account. This metric was already proposed by the author some years ago under the name square-root integral or SQRI (Barten, 1987, 1989, 1990), but without the complete background given here.

In this chapter, the validity of the various metrics will be tested by a principal analysis, where the functional parameters of these metrics are compared with the requirements for a good representation of perceived image quality. Furthermore, the metrics will also be tested by a comparison of the image quality predicted by these metrics for different MTFs with the image quality obtained with these MTFs from subjective image quality ratings.

\subsection{Nonlinear effect of modulation}

Granger \& Cupery (1972) noticed in an investigation on the image quality of photographic pictures, where several different pictures with the same content were compared, that there is a linear correlation between perceived image quality of these pictures and the number of just-noticeable differences between these pictures. As the difference between these pictures mainly consisted of a difference in the modulations occurring in these pictures, the equations for contrast discrimination given in the previous chapter can be used to calculate the number of discriminable modulation steps for these pictures. By using Eq. (7.15) for each possible modulation, starting from zero modulation, and adding the so obtained modulation difference to the previous modulation, etc., one can calculate the number of just-noticeable differences or jnds as a function of the modulation. If this is done with the aid of the generalized expression given by Eq. (7.17), where the modulation is expressed in normalized units, the results are independent of spatial frequency, luminance and field size. They are shown in Fig. 8.1 for the typical $k$ value 3 . The calculation gives only data for an integer number of discrimination levels, but they are connected in this figure by a continuous curve. Apart from the value chosen for $k$, for which the typical value was used, this curve has general validity. Fig. 8.2 shows the same curve, but plotted as a function of the square root of the normalized modulation. From this figure, it appears 


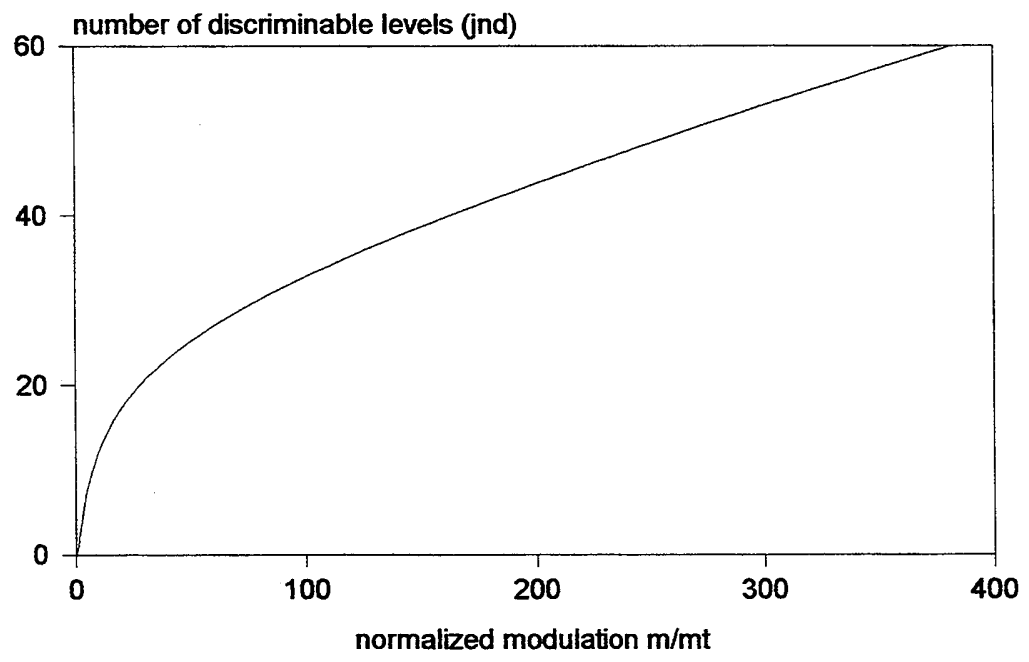

Figure 8.1: Number of discriminable modulation levels (jnds) of a sinusoidal luminance pattern as a function of the normalized modulation $\mathrm{m} / \mathrm{m}_{\mathrm{t}}$. The curve has been obtained by a step by step summation of Eq. (7.17) for $k=3$.

that the number of discriminable levels increases about linearly with the square root of the modulation. The dashed curve in this figure represents the approximation by a square-root relation. A power law for contrast discrimination was already mentioned

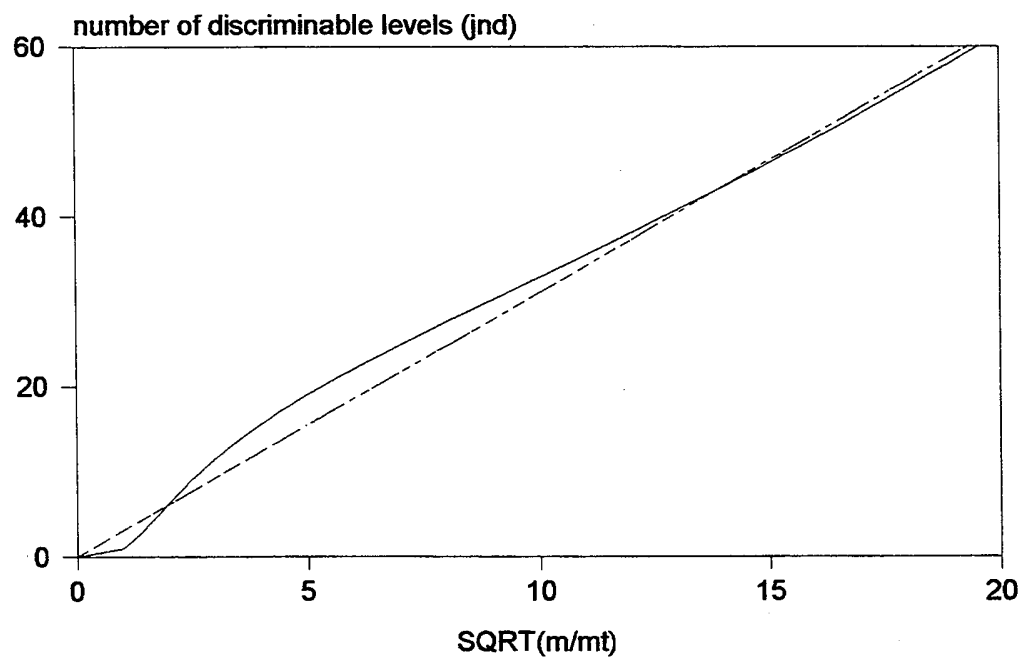

Figure 8.2: Solid curve: same as in Fig. 8.1, but plotted as a function of the square root of the normalized modulation. Dashed line: approximation with a linear relation between the number of discriminable modulation levels and the square root of the normalized modulation. 
by Legge (1981), based on measurements at low and medium modulation levels. From Fig. 8.2 where the modulation is extended to much higher modulation levels, it appears that the power law is approximately a square-root law. For normal images where the modulation of the spatial frequency components generally extends over a large range of modulations, the square-root relation forms a very good basis for an image quality metric. A more precise description has no sense, as the modulations of the various spatial frequency components of an image are arbitrarily distributed over the whole range, which cancels possible deviations. Therefore, the square root relation will be used here for the description of the nonlinear behaviour of the visual system above the threshold.

This relation is also valid for the visually perceived contrast of sinusoidally modulated luminance patterns. Published measurements of this relation give usually the exponent of the relation between the logarithm of the visually perceived contrast and the logarithm of the physical modulation. However estimating the perceived modulation is very difficult, because it is not very well defined. Furthermore, these estimations are sometimes also influenced by a knowledge of the observers about the physical contrast of the object. Therefore, considerable differences of this exponent occur in publications of experimental investigations. The most reliable experiments in this respect have been made by Cannon (1985). He used six logarithmic spaced modulation levels between $1 \%$ and $80 \%$ modulation and four different spatial frequencies: $2,4,8$, and 16 cycles/deg. The luminance was $100 \mathrm{~cd} / \mathrm{m}^{2}$ and the field size was circular with a diameter of $2^{\circ}$. The average of the perceived contrast was obtained from the estimates of nine observers at two presentations. Because each

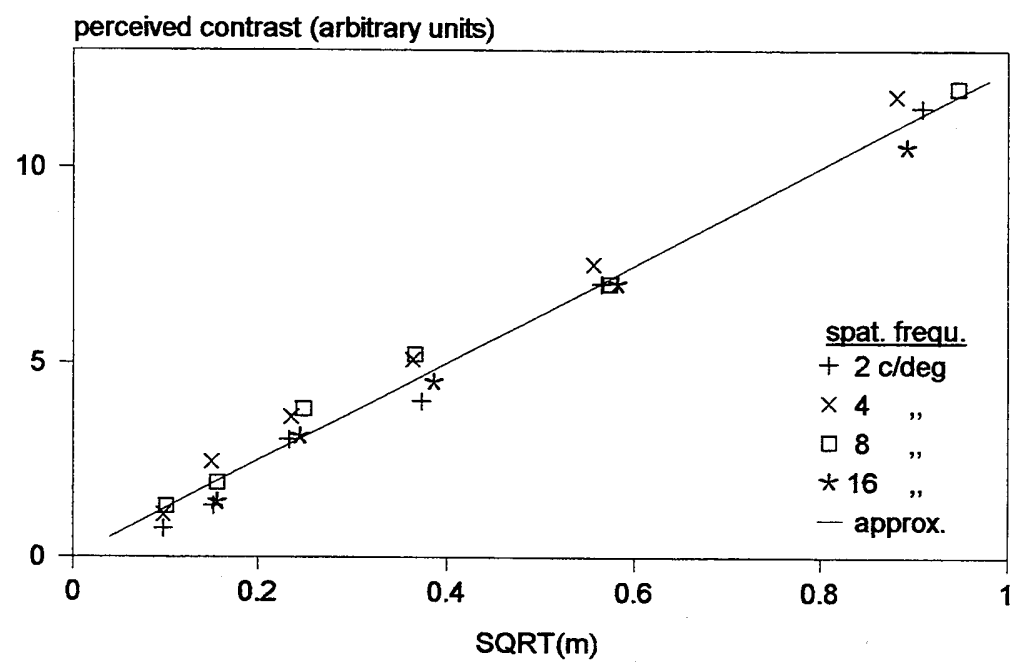

Figure 8.3: Perceived contrast as a function of the square root of the modulation for measurements by Cannon (1985) with four different spatial frequencies. The solid line shows the approximation with a linear relation between the quantities along both axes. 
subject could use a different scale for his subjective estimate, the estimates were first normalized to a common mean of all estimates. The so obtained results expressed in arbitrary units are shown in Fig. 8.3 where a linear scale is used for the perceived contrast and the modulation is plotted with a square root scale. Although the modulation threshold for the different spatial frequencies is different, they nearly coincide with a linear curve for the quantities along both axes. The perceived contrast appears to be about equal at the maximum modulation and about equal at the minimum modulation where it approaches to zero. This is in good agreement with measurements by Watanabe et al. (1968) who found similar results for measurements with equally perceived contrast at different spatial frequencies. If the perceived contrast would be plotted as a function of the normalized modulation, the data would no longer approximately coincide on a common curve because of the difference in modulation threshold for the different spatial frequencies. However, it appears that they can be brought to a nearly common curve again by dividing the perceived contrast by the square root of the modulation threshold. This is shown in Fig. 8.4. The coincidence of the data with a common linear curve for all data is even more pronounced than in Fig. 8.3. The data shown in Fig. 8.4 form an extra support for the use of the square root of the normalized modulation as the functional parameter for the judgment of image quality.

Contrary to measurements where the object consists of a single sinusoidal luminance pattern, normal images consist of a combination of sinusoidal luminance patterns with different modulations and spatial frequencies. From the data showed in

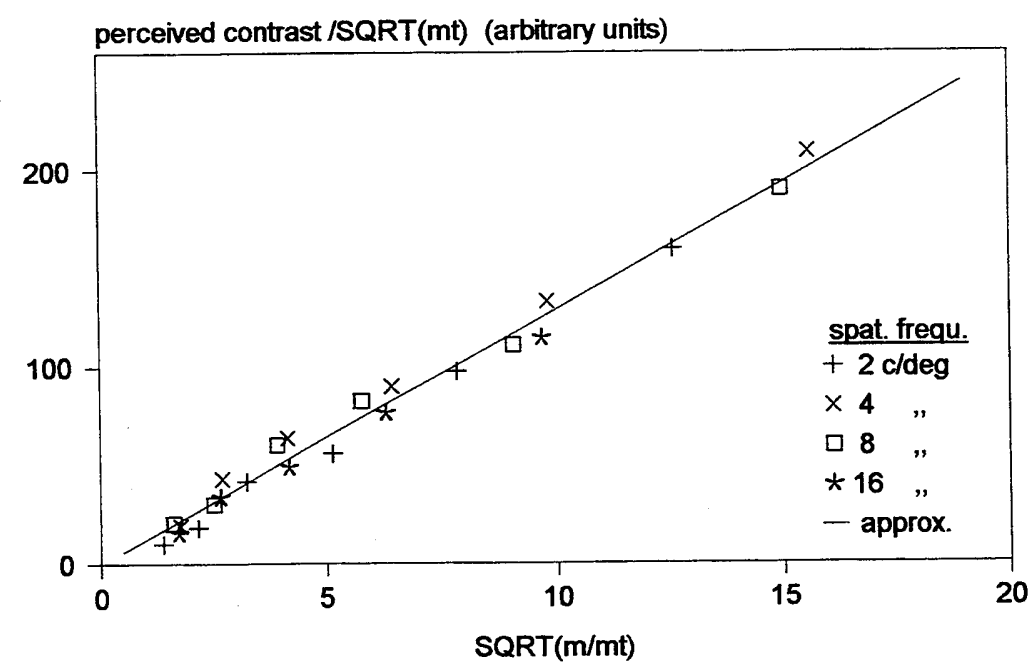

Figure 8.4: Perceived contrast divided by the square root of the modulation threshold as a function of the square root of the normalized modulation for the measurements given in Fig 8.3. The solid line shows the approximation with a linear relation between the quantities along both axes. 
Figs. 8.4, it may be concluded that the perceived contrast increases linearly with the square root of the normalized modulation. Furthermore, the calculated curve in Fig. 8.2 shows that also the number of discriminable modulation levels increases approximately linearly with the square root of the normalized modulation. As Granger and Cupery found a linear relation between perceived image quality and just-noticeable differences, it may be concluded that the perceived quality of an image is linearly related with the square root of the normalized modulation. This means that the perceived image quality is linearly related with the square root of the modulation divided by the modulation threshold or multiplied with the contrast sensitivity. Although the contrast sensitivity of the eye is only defined at threshold level, it appears to play also an important role at suprathreshold levels of modulation.

The modulations of the different spatial frequency components of an image are generally multiplied with the modulation transfer function or MTF of the imaging system. At low spatial frequencies the MTF is usually about 1 and at high spatial frequencies it decreases with spatial frequency. For the use of the square root of the normalized modulation in a metric for image quality, the contributions of the various spatial frequency components of an image have still to be integrated over the spatial frequency spectrum to obtain the totally perceived image quality. For the way in which these contributions have to be integrated, much can be learned from the various image quality metrics developed in the past decennia.

\subsection{Image quality metrics}

Image quality metrics are usually generic measures. This means that they are independent from the actual picture content. They do not contain the modulation of the different spatial frequency components of an actual image, but only the MTF by which these modulations are multiplied. This is almost remarkable, but in practice, it appears that the real amplitude of these components does not play an important role in the judgment of image quality. The amplitude of the components is obviously taken into account as a generic quantity in the distribution of the different spatial frequency components over the spatial frequency spectrum.

As sharpness has always been one of the most important aspects of image quality, early attempts for image quality metrics were concentrated on resolution. This resolution was usually expressed in a number of lines that could just be distinguished. As this number describes only the maximum resolution of an image, it can only be used as a comparative measure of resolution for types of images that are further similar in other aspects. A more precise definition of resolution became possible with the introduction of the MTF in the early fifties, which was mainly due to the pioneering work of Schade (1951-1955). With the aid of the MTF, a more 
exact description could be given of the sharpness reduction at high spatial frequencies.

\subsubsection{Modulation transfer area (MTFA)}

However, for a good description of image quality also the contrast sensitivity of the eye has to be taken into account. One of the first attempts to take also the contrast sensitivity of the eye into account was made by Charman \& Olin (1965). They introduced an image quality metric called modulation transfer area or MTFA. The use of this metric was further promoted by Snyder (1973). In this metric the surface area between the MTF and the modulation threshold as a function of spatial frequency is used as a measure of image quality. The MTFA is given by

$$
A=\int_{0}^{u_{\max }}\left\{M(u)-m_{t}(u)\right\} \mathrm{d} u
$$

where $M(u)$ is the MTF of the imaging system, $m_{\mathrm{t}}(u)$ is the modulation threshold of the eye, and $u_{\max }$ is given by the condition

$$
M\left(u_{\max }\right)=m_{t}\left(u_{\max }\right)
$$

The unit of this metric is spatial frequency. The metric is based on the idea that a modulation below the threshold cannot contribute to visual perception. Although the MTFA is widely used as a measure for image quality and is still used as official standard in the USA, it lacks a good correlation with subjectively perceived image quality. This is partly because it is linearly related with the modulation, instead of with the quare root of it, and that the modulation threshold is subtracted from the MTF, instead of dividing the MTF by it.

\subsubsection{Integrated contrast sensitivity (ICS)}

To overcome the last of these two disadvantages, van Meeteren (1973) proposed a metric called integrated contrast sensitivity or ICS. In this metric the modulation threshold is not subtracted from the MTF, but the MTF is multiplied by the contrast sensitivity of the eye, which means that it is divided by the modulation threshold. The ICS is given by

$$
I=\int_{0}^{\infty} M(u) S(u) \mathrm{d} u
$$

where $S(u)$ is the contrast sensitivity function. Similarly as with the MTFA, the image quality is expressed in spatial frequency units. Although the ICS is already an improvement over the MTFA, it is still depends linearly on modulation. 


\subsubsection{Subjective quality factor (SQF)}

In the MTFA and the ICS, the contribution of the different spatial frequency components to the total image quality is taken into account by linear integration over spatial frequency. Granger \& Cupery (1972) found that logarithmic integration shows a better correlation with the perceived image quality. They introduced a metric called subjective quality factor or SQF. In this metric a logarithmic integration over spatial frequency is used. Similarly as with the ICS, the MTF is multiplied by the contrast sensitivity function, but the contrast sensitivity function is simplified to a rectangular function with a value 1 between 3 and 12 cycles/deg and a value zero outside this range. The SQF is given by

$$
Q=K \int_{u_{1}}^{u_{2}} M(u) \mathrm{d}(\log u)
$$

where $u_{1}=3 \mathrm{cycles} / \mathrm{deg}, u_{2}=12 \mathrm{cycles} / \mathrm{deg}$, and $K$ is a normalizing constant equal to $1 / \log 4$ to normalize the SQF to a value 1 for the ideal situation that $M(u)=1$ over the total integration range. In this way, the image quality is expressed in dimensionless units. Although the logarithmic integration is an improvement over a linear integration, the dependence on modulation is still linear. Furthermore, the representation of the contrast sensitivity function by a rectangular function forms an oversimplification of the actual situation, as was found later by Higgins (1977).

\subsubsection{Discriminable difference diagram (DDD)}

As has already been mentioned in the section 8.2, Granger and Cupery also found in their investigation, that there is a linear correlation between subjective image quality and just-noticeable differences. Carlson \& Cohen (1980) used this principle for the development of an image quality metric based on the number of discriminable modulation differences in different spatial frequency areas. Instead of a logarithmic integration over the spatial frequencies, they applied a logarithmic summation by using logarithmic spaced spatial frequency channels with a width of a factor two. The discriminable modulation levels of each channel are indicated in a discriminabledifference diagram or DDD. The total number of just-noticeable differences under the MTF of the imaging system gives the resulting image quality. They assumed that a difference of one unit of this number is equal to a difference of one jnd in image quality. The results of their image quality metric are, therefore, expressed in units of just-noticeable differences. Although the use of the jnd as unit for image quality is an improvement over the use of other units, the method is not very practical. For every condition of luminance and field size, a different diagram has to be used. The constants required for the calculation of these diagrams have to be obtained from measurements at these conditions. Although the dependence on modulation is nonlinear, the DDD does not always show a good correlation with perceived image 
quality. Furthermore, the just-noticeable difference derived from the sum of the discriminable differences in the different spatial frequency channels appears not to correspond with the just-noticeable difference between two images.

\subsubsection{Square-root integral (SQRI)}

The assumptions made in section 8.2 will now be used here as basis for an image quality metric where the nonlinear behaviour of the eye is taken into account. This metric is called square-root integral or SQRI and was already proposed by the author some years ago (Barten 1987, 1989, 1990) without the background given in this chapter. This metric is based on the assumption that the perceived image quality is linearly related with the number of just-noticeable modulation differences and that this number is linearly related with the square root of the modulation divided by the threshold modulation (See section 8.2). Furthermore, use is made of the fact that the modulation of the different spatial frequencies of an image can be represented by the multiplication factor formed by the MTF of the imaging system, as is done in other metrics, and a logarithmic integration over the spatial frequencies is used, as was done by Granger and Cupery in their SQF metric (See section 8.3.3). The metric is expressed in units of just-noticeable differences or jnds, as was done by Carlson and Cohen in their DDD metric (See section 8.3.4). The so obtained SQRI metric is given by

$$
J=\frac{1}{\ln 2} \int_{u_{\min }}^{u_{\max }} \sqrt{\frac{M(u)}{m_{\mathrm{t}}(u)}} \frac{\mathrm{d} u}{u}
$$

where $\mathrm{d} u / u$ stands for the logarithmic integration over spatial frequency. The constant in front of the integral has been chosen so, that one unit of the SQRI corresponds indeed with one just-noticeable difference in image quality. To obtain this, the constant has been determined from a comparison with measurement data published by Carlson \& Cohen (1980). The constant appeared to be about 1.4 or about $1 / \mathrm{ln} 2$. See Barten (1987). Although the choice of $1 / \mathrm{ln} 2$ for this constant has no physical meaning, it may be useful for an interpretation of the results. It says that the SQRI increases with one unit when the integrand increases with one unit in a spatial frequency band with a width of a factor two. This is the width of the spatial frequency channels used by Carlson and Cohen in their DDD metric. The jnd, which was already introduced by Carlson and Cohen, is a very important unit for image quality, because it is a basic unit of perception. It has further the advantage over other possible units that it enables a good interpretation and comparison of the results. A difference of one jind corresponds with $75 \%$ correct response in a 2 AFC experiment.

In the SQRI, integration limits are used to restrict the integration to spatial frequency areas that can contribute to the image quality. For television images, for 
instance, the available spatial frequency area is limited by the bandwidth of the television system. Higher spatial frequencies cannot contribute to the perceived image quality. For the same reason also a lower limit has been introduced. This limit is caused by the finite spatial dimensions of the image. When the frequency spectrum is written as a Fourier sum, the lowest term of this sum has a frequency $1 / X$, where $X$ is the size of the image. With an assumed bandwidth $1 / X$ for each term in the continuous representation used here, the lowest frequency is $0.5 / X$. This means that for $u_{\text {min }}$, half the inverse of the image size has to be used.

\subsection{Two-dimensional aspects}

A remarkable aspect of the image quality metrics discussed in the previous section is, that they are expressed by a one-dimensional equation. One would have expected a two-dimensional equation because of the two-dimensional character of an image. The one-dimensional form of these metrics is, however, only apparent. They can also be written in a two-dimensional form. If the one-dimensional expression is represented by an integral of the form

this integral can be written as

$$
\int F(u) \mathrm{d} u
$$

$$
\frac{1}{2 \pi} \int F(u) \frac{2 \pi u \mathrm{~d} u}{u}
$$

One can now convert this in a two-dimensional expression by replacing $2 \pi u d u$ by $\mathrm{d} u \mathrm{~d} v$ and $1 / u$ by $1 / \sqrt{ }\left(u^{2}+\nu^{2}\right)$. This transforms the one-dimensional integral in

$$
\frac{1}{2 \pi} \iint F(u, v) \frac{\mathrm{d} u \mathrm{~d} v}{\sqrt{u^{2}+v^{2}}}
$$

where the function $F(u)$ has been replaced by the function $F(u, v)$ that may be different for the two dimensions. From Eq. (8.6) also a different two-dimensional expression can be derived by using polar coordinates. This gives

$$
\frac{1}{2 \pi} \int_{0}^{2 \pi}\left\{\int F(u, \theta) \mathrm{d} u\right\} \mathrm{d} \theta
$$

This expression is equivalent to an averaging of the one-dimensional integral over different directions. In practice, four directions are already sufficient: $0^{\circ}, 90^{\circ},+45^{\circ}$, and $-45^{\circ}$. Images are usually isotropic and the contrast sensitivity of the eye is also almost isotropic, so that usually the one-dimensional form of the metric can be used. 


\subsection{Functional analysis of image quality metrics}

By making use of the linear relation between perceived image quality and justnoticeable differences, different image quality metrics can be analyzed with respect to their effect on image quality. For this analysis, image quality measurements will be used where the image quality is varied in steps of one just-noticeable difference.

For this analysis, the equation for an image quality metric is written in the following general form:

$$
J=\int j(u) \mathrm{d}(\ln u)
$$

where $J$ stands for the total image quality expressed in jnds and $j(u)$ is the image quality contribution per logarithmic spatial frequency interval d(lnu). For practical reasons a logarithmic spatial frequency interval has been chosen, although a linear interval $\mathrm{d} u$ or a quadratic interval $\mathrm{d} u^{2}$ would also have been possible. From the equations for the various metrics given in the previous sections, the following expressions can be derived for the distribution function $j(u)$ :

for the MTFA

for the ICS

$$
j(u) \propto\{M(u)-1 / S(u)\} u
$$

for the SQF

$$
j(u) \propto M(u) S(u) u
$$

$$
j(u) \propto \frac{1}{\ln 4} M(u) \text { for } 3 \mathrm{c} / \mathrm{deg}<u<12 \mathrm{c} / \mathrm{deg}, \text { else } j(u)=0
$$

and for the SQRI

$$
j(u)=\frac{1}{\ln 2} \sqrt{M(u) S(u)}
$$

With exception of the SQRI, the functions $j(u)$ still have to be multiplied with a certain factor to express $J$ in jnds.

If the focus condition of an image is varied in steps of one just-noticeable difference, the function $j(u)$ can be determined and be compared with the above given expressions. In Fig. 8.5 a schematic drawing is given for the MTFs of two focus conditions that differ one jnd in image quality. The spatial frequencies where the MTFs have decreased with $50 \%$ are $u_{1}$ and $u_{2}$. If the MTFs are approximated by rectangular functions with a width given by these frequencies, the function $j(u)$ can be derived from a measurement of $u_{1}$ and $u_{2}$ with the aid of the following expression:

$$
j(u)=\frac{d J}{d(\ln u)} \approx \frac{\Delta J}{\Delta(\ln u)}=\frac{1}{\ln u_{2}-\ln u_{1}}=\frac{1}{\ln \left(u_{2} / u_{1}\right)}
$$




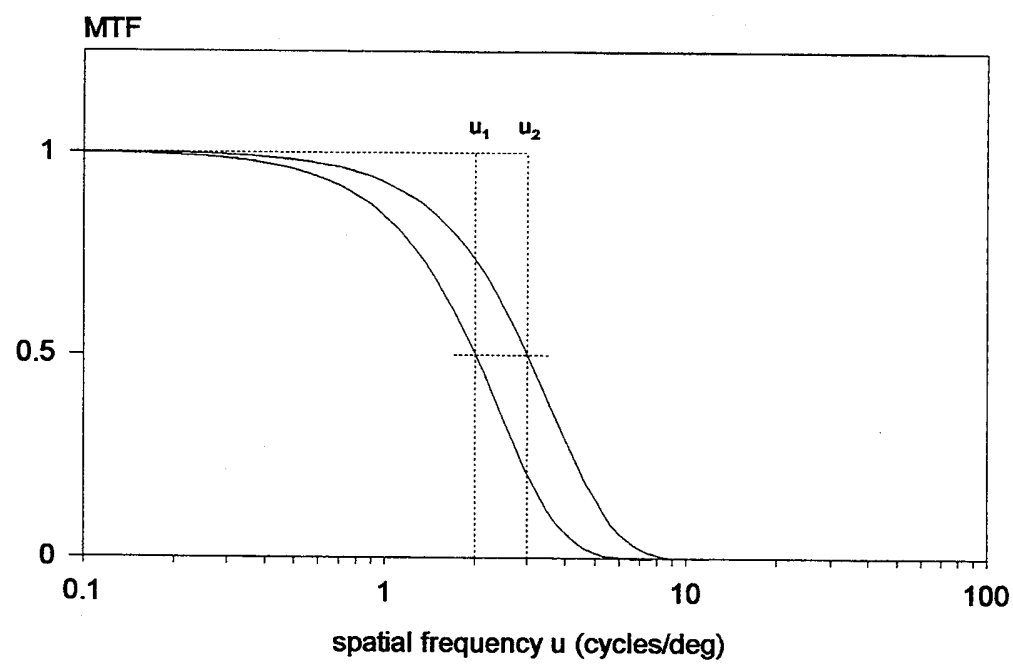

Figure 8.5: Schematic drawing of two MTFs that give a difference of one jnd in image quality. These MTFs can be idealized by rectangular functions with a width determined by the spatial frequencies $u_{1}$ and $u_{2}$ where the MTFs have decreased with $50 \%$.

For the spatial frequency $u$ in $j(u)$ the geometric mean of $u_{1}$ and $u_{2}$ may be used. This experiment has to be repeated for several values of $u_{I}$ to get the image quality contribution for a range of values of the spatial frequency spectrum.

Fig. 8.6 shows the results obtained from an experiment with this type of measurements made by Carlson \& Cohen (1980). They varied the focus condition of projected slides corresponding with a just-noticeable change in sharpness over a wide range of focus conditions and measured the spatial frequency where the MTFs had decreased to $50 \%$. The MTFs had a Gaussian shape. The average luminance was 111 $\mathrm{cd} / \mathrm{m}^{2}(35 \mathrm{~mL})$ and the field size was $25.6^{\circ} \times 25.6^{\circ}$. The images consisted of a crowd scene, a portrait of a woman's face in colour, and a monochrome version of the same picture. The first picture contained many small details, whereas the two other pictures had large smooth transitions. Although the data points in Fig. 8.6 show a considerable scattering, their general behaviour is consistent and shows no systematic difference between the different picture types. This confirms the assumption that the perceived image quality can be described by a generic quantity, which does not depend on the actual picture content. The solid curve through the data points gives the SQRI prediction calculated with the aid of Eq. (8.14). This curve agrees very well with the average of the data points.

The same type of measurements was made by Carlson \& Cohen (1978) with projected slides of an airplane cockpit display. The average luminance was $6.4 \mathrm{~cd} / \mathrm{m}^{2}$ $(2 \mathrm{~mL})$ and the field size was $7.2^{\circ} \times 7.2^{\circ}$. Fig. 8.7 shows the results obtained from 


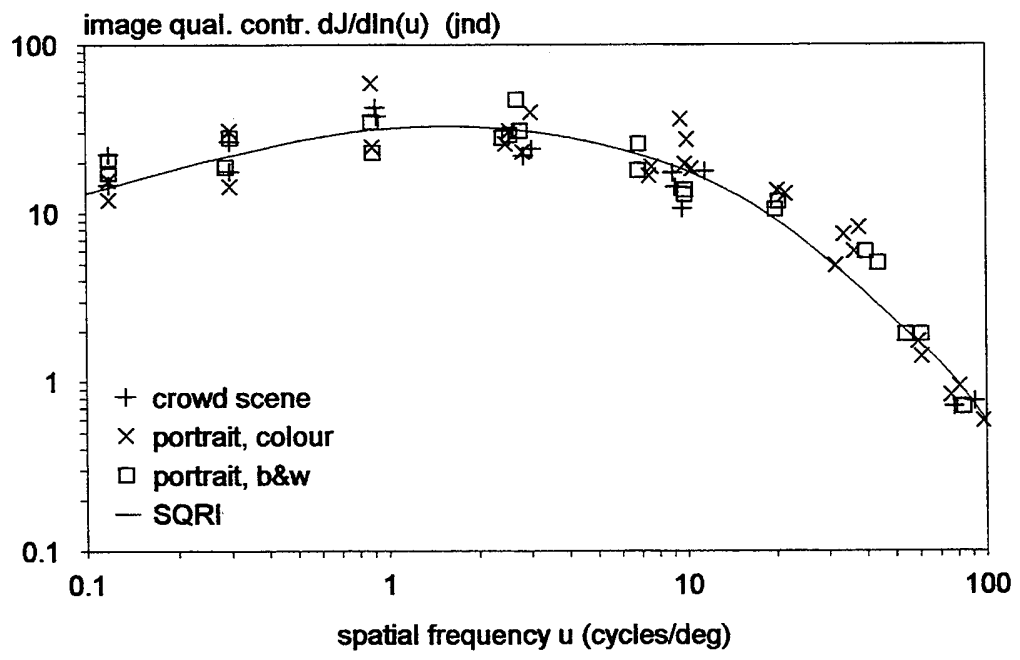

Figure 8.6: Image quality contribution of different spatial frequency areas calculated with Eq. (8.15) for measurements by Carlson \& Cohen (1980). The different symbols represent different types of images. The data show no systematic difference for the different types of images. The solid curve through the data points gives the SQRI prediction calculated with the aid of Eq. (8.14).

these measurements. Although the picture is the image of an artificial object, it shows about the same behaviour as the natural images in Fig. 8.5. The solid curve through

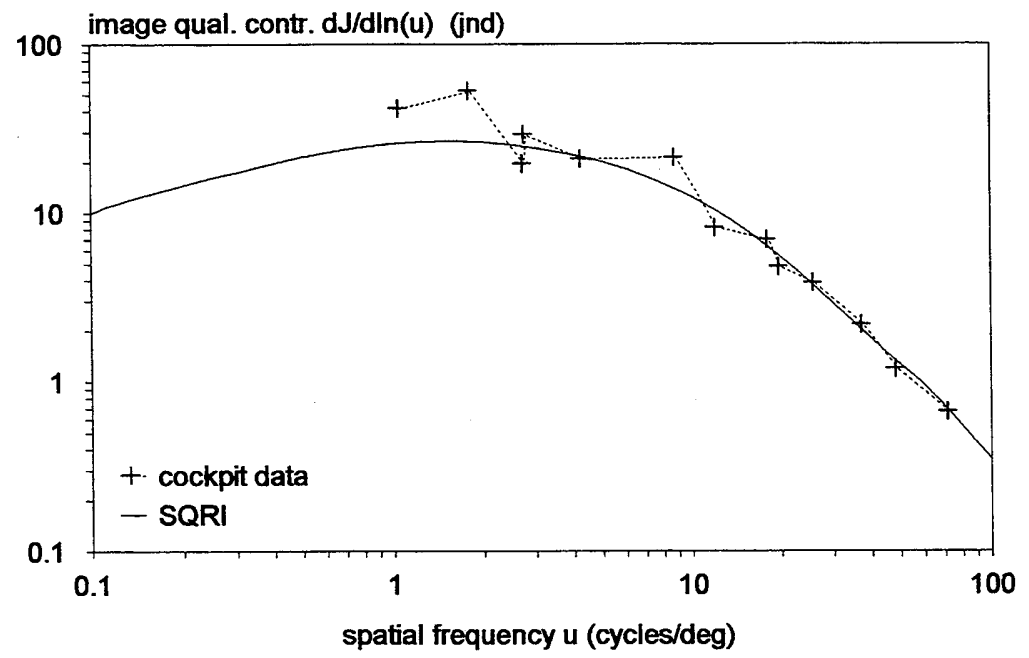

Figure 8.7: Same as Fig . 8.6, but for an image with artificial data of an airplane cockpit display. The solid curve through the data points gives the SQRI prediction calculated with the aid of Eq. (8.14). The similarity with Fig. 8.6 shows that there is no effect of image content on the perceived image quality. 
the data points gives the SQRI prediction calculated with the aid of Eq. (8.14). This curve too agrees very well with the data points.

In Fig. 8.8 the same data are shown as in Fig. 8.6, but plotted with a linear vertical scale, instead of a logarithmic scale and using only the geometric means of the data, as the spread of the data points would have increased considerably by plotting them on a linear scale. Besides the image quality contribution predicted by the SQRI, also the predictions by the MTFA, the ICS and the SQF metrics are shown. For these metrics arbitrary factors have been used to convert the units to jnds. The use of a linear vertical scale in this figure makes that the surface area under the curves corresponds with the total image quality. Therefore, the figure gives a good impression of the relative importance of the different spatial frequency areas. From the figure, it can be seen that the MTFA gives an underestimation of the image quality contribution of low spatial frequencies and a strong overestimation of the image quality contribution of high spatial frequencies. The ICS gives a much better estimate at high frequencies than the MTFA, but still gives an underestimation of the image quality contribution of low spatial frequencies. The SQF uses only a small part of the total spatial frequency range and gives, therefore, an underestimation of low spatial frequency areas and high spatial frequency areas. The SQRI clearly shows the best agreement with the results obtained from the measurements.

Only a few measurements are available where the image quality is changed in

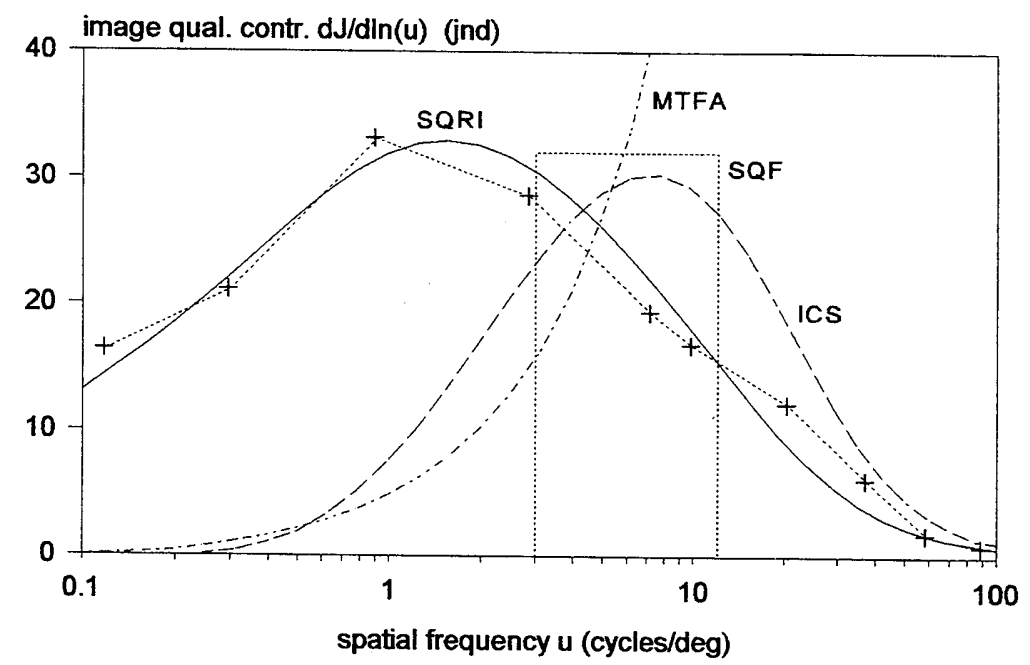

Figure 8.8: Same data as in Fig. 8.4, but plotted with a linear vertical scale and using only the geometric mean of the data. In addition to the image quality contribution predicted by the SQRI, also the predictions by the MTFA, ICS, and SQF metrics are shown. These contributions have been multiplied by arbitrary factors to convert the units to jnds. The area below the curves represents the total image quality. 
steps of one just-noticeable difference. In experiments by other investigators, the focus condition of the image was changed by an electronic blurring process. The difference in focus was measured by determining the change of the sigma of the linespread function used in the blurring process. For a Gaussian line-spread function with a standard deviation $\sigma$, the MTF is given by

$$
M(u)=e^{-2 \pi^{2} \sigma^{2} u^{2}}
$$

From this equation follows for the spatial frequency where the MTF is $50 \%$

$$
u_{0.5}=\sqrt{\frac{\ln 2}{2}} \frac{1}{\pi \sigma}
$$

With this equation the values of $u_{1}$ and $u_{2}$ used in Eq. (8.15) can be obtained. Replacing these values by the corresponding sigma values $\sigma_{1}$ and $\sigma_{2}$ gives

$$
j(u)=\frac{1}{\ln \left(\sigma_{1} / \sigma_{2}\right)}
$$

The value of $u$ in $j(u)$ can be calculated with Eq. (8.17) by using the geometric mean of $\sigma_{1}$ and $\sigma_{2}$.

Fig. 8.9 shows the so obtained data of the distribution function for measurements by Baldwin (1940). He measured the just-noticeable focus difference of projected movie pictures at different focus conditions. The average luminance was 7 $\mathrm{cd} / \mathrm{m}^{2}$ and the field size was $14.5^{\circ} \times 13.7^{\circ}$. The just-noticeable differences were obtained from a measurement of the psychometric function. The solid curve in the figure represents the SQRI prediction calculated with Eq. (8.14). This curve agrees

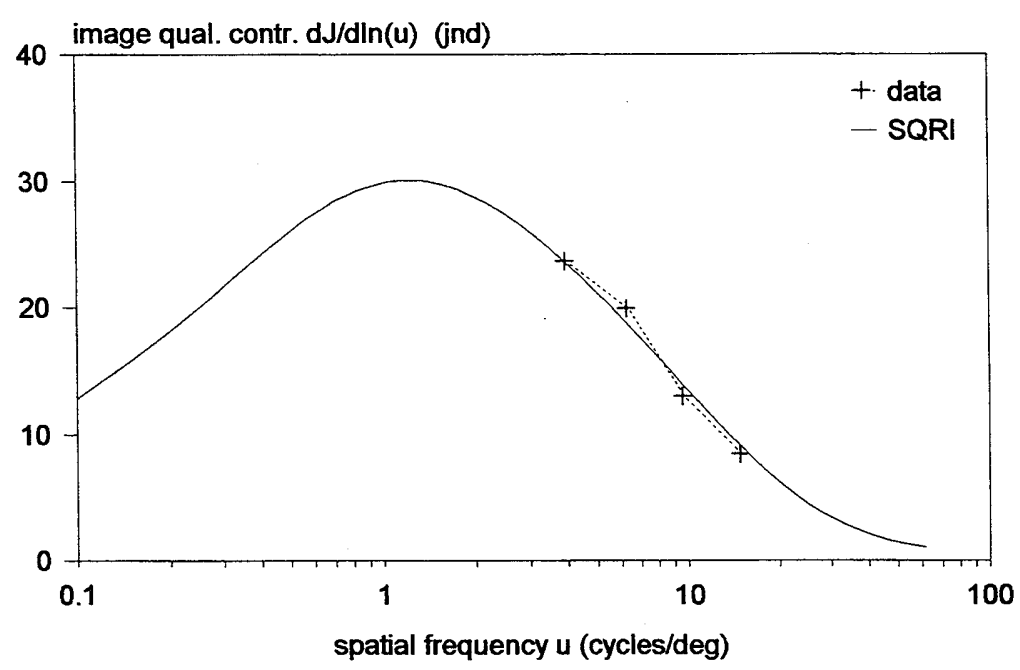

Figure 8.9: Image quality contribution of different spatial frequency areas calculated with Eq. (8.18) for measurements of defocused movie pictures by Baldwin (1940). The solid curve gives the SQRI prediction calculated with the aid of Eq. (8.14). 


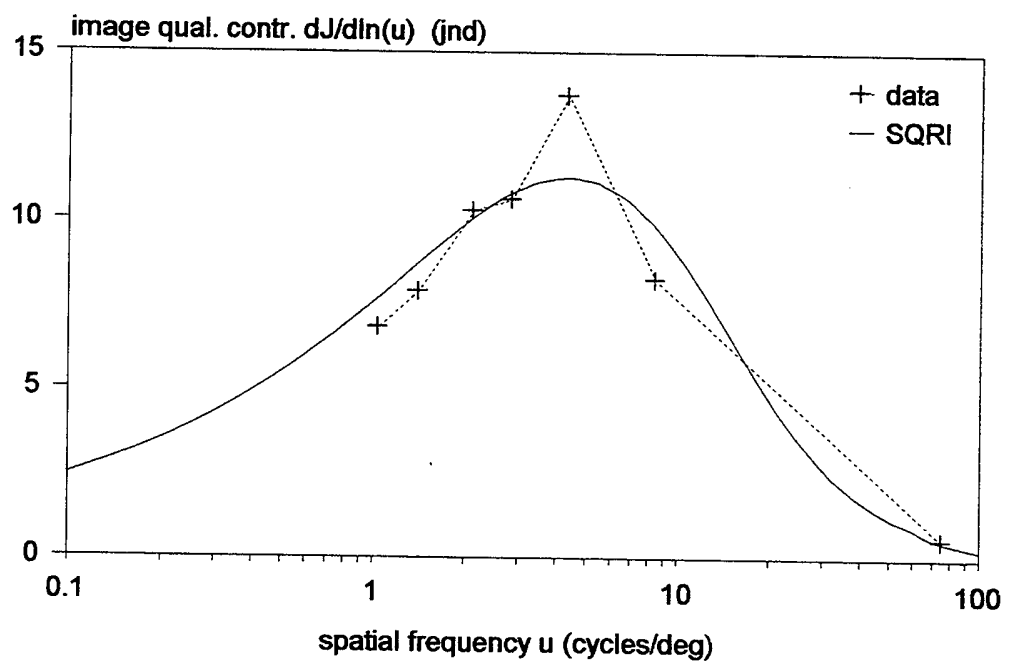

Figure 8.10: Image quality contribution of different spatial frequency areas calculated with Eq. (8.18) for measurements of a blurred edge transition by Watt \& Morgan (1983). The solid curve gives the SQRI prediction calculated with the aid of Eq. (8.14).

very well with the data. The use of the psychometric function for the measurements has probably caused that the deviations are very small. Predictions by the other image quality metrics have been omitted, because they show the same kind of deviations as in Fig. 8.8.

Fig. 8.10 shows similar results for measurements made by Watt \& Morgan (1983). They used band-shaped images consisting of a single edge transition displayed on a CRT monitor. The average luminance was $292 \mathrm{~cd} / \mathrm{m}^{2}$ and the field size was $3^{\circ} \times 0.2^{\circ}$. The images were electronically blurred in the direction of the transition. The just-noticeable differences were determined with a 2AFC method. The data points are the average for two subjects. In this figure too, the solid curve through the data points has been calculated only for the SQRI. The different shape of the curve compared with the shapes of the curves in Figs. 8.5 and 8.6 is caused by the small field size in vertical direction. With a few exceptions, the position of the data points agrees well with the SQRI prediction.

In the given experiments only the spatial frequency was changed. At these frequencies, it was assumed that the MTF dropped from a value $I$ to a value zero for the hypothetical situation of a rectangular MTF. The question remains how the image quality would vary if the spatial frequency is constant and only the modulation or the MTF is changed. In the MTFA, ICS and SQF metrics a linear relation is assumed, whereas in the SQRI a square-root relation is assumed. 


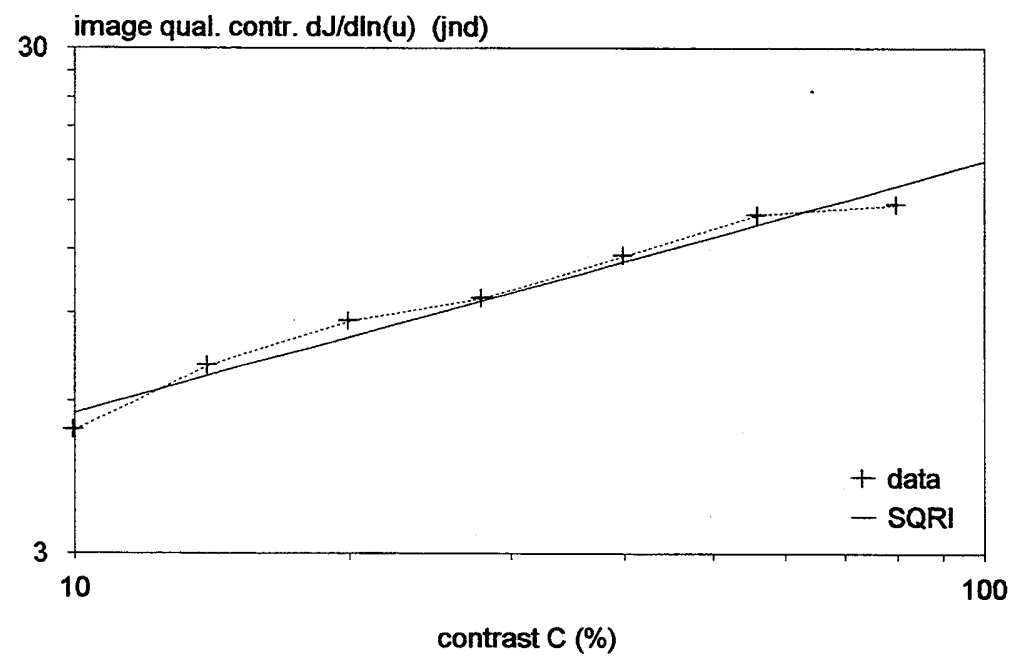

Figure 8.11: Image quality contribution as a function of contrast calculated with Eq. (8.18) for measurements of a blurred edge transition by Watt \& Morgan (1983). The data show a square-root dependence on contrast. The solid line through the data gives the SQRI prediction calculated with Eq. (8.14) after multiplying $M$ with the contrast $C$.

Watt and Morgan made an experiment in a second part of their investigation, where they changed the contrast at a fixed focus condition and measured the justnoticeable change in focus as a function of contrast. In the same way as for their other experiment, the image quality contribution $j(u)$ could be derived from these measurements, but now as a function of contrast at a fixed spatial frequency. This spatial frequency could be calculated from the average sigma of the blur and appeared to be $4.5 \mathrm{cycles} / \mathrm{deg}$. The results for the average data of two observers are shown in Fig. 8.11. The data points clearly show a square-root dependence on contrast. The solid line through the data points represents the prediction by the SQRI calculated with Eq. (8.14) after multiplying $M$ with the contrast factor $C$. The SQRI prediction shows a very good agreement with the data. The square-root dependence on contrast of the data points over a large range of contrast values confirms the validity of the square-root approximation. The MTFA, ICS and SQF predictions are not given in the figure. They would have shown a linear dependence on contrast.

\subsection{Effect of differently shaped MTFs}

In the previous section, different image quality metrics have been compared with respect to their effect on image quality by making use of the linear relation between perceived image quality and the number of just-noticeable differences. From the given analysis, it might be clear that most image quality metrics will not show a good 
agreement with actually perceived image quality. One may wonder why these metrics sometimes still can show a good correlation with perceived image quality. The reason is, that MTFs often have a similar, for instance, Gaussian shape, so that the comparison between different images is limited to a small type of variations. Under these conditions, even the worst image quality metric can show a good correlation with perceived image quality. If the spread of the measurement data is very large, even a fundamentally better metric can hardly improve the correlation. Correlation coefficients say often more about the spread in the measurement data than about the quality of the metric.

However, more difference between different metrics occurs, when images are compared that are displayed with MTFs with different shapes. Experiments with such MTFs have been made by Higgins (1977). He measured the subjective image quality of four photographic images that were each displayed with 22 different MTFs. Some of these MTFs had a quite irregular shape, like the examples shown in Fig. 8.12. The images had a size of $10 \times 10 \mathrm{~cm}$ and were viewed at a distance of $0.38 \mathrm{~m}$, corresponding with a field size of $15^{\circ}$. The reported data are the averages of the judgments by 20 observers. Figs. 8.13 through 8.16 show comparisons of these image quality judgments with the image quality calculated with the MTFA, ICS, SQF, and SQRI, respectively. For all four metrics a linear regression has been made between the measurements and the calculations. The solid lines in the figures are the regression lines. The $\mathrm{R}^{2}$ value of the correlation between measurements and calculations was $63.0 \%$ for the MTFA, $93.3 \%$ for the ICS, $89.3 \%$ for the SQF and $99.5 \%$ for the SQRI. This order of succession roughly corresponds with the difference in image

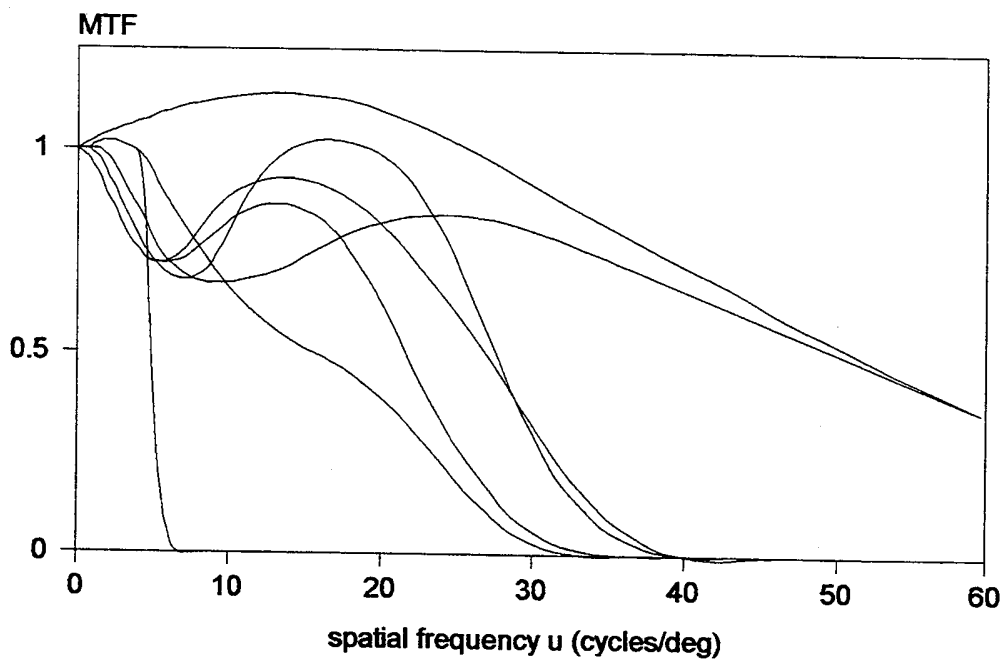

Figure 8.12: Seven of the 22 different MTFs used by Higgins (1977) in his investigation for a comparison of image quality metrics. From the examples shown here, some have a very irregular shape. 


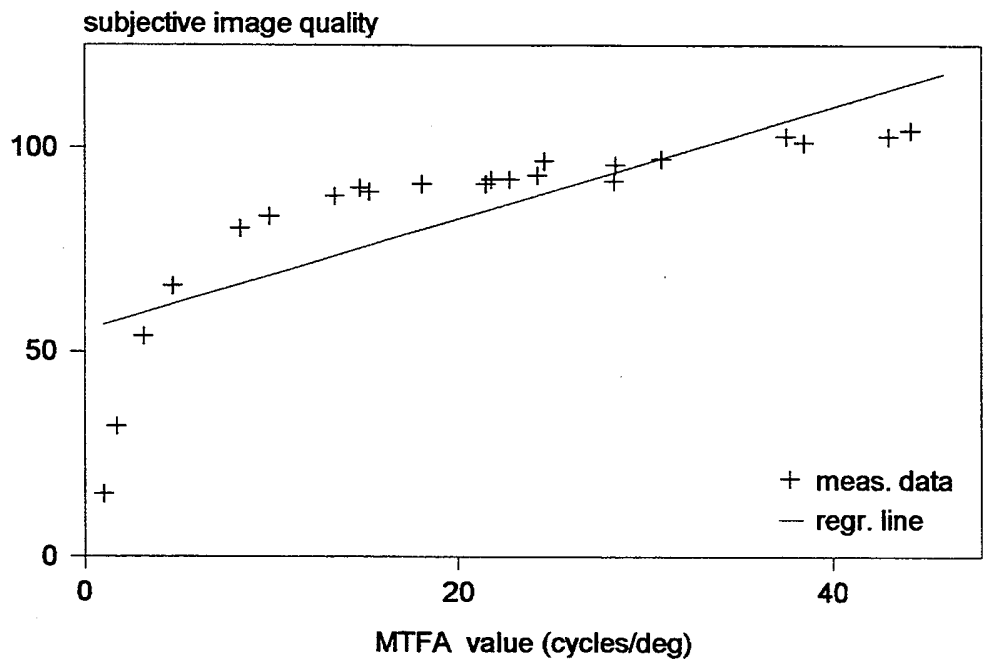

Figure 8.13: Measured subjective image quality as a function of MTFA value for photographs reproduced with 22 different MTFs from an investigation by Higgins (1977). The solid line is the linear regression line. Correlation between measurements and calculations $63.0 \%$.

quality contribution shown in Fig. 8.8. Fig 8.16 shows that the relation between measurements and calculations for the SQRI is strictly linear over the very large variation of the image quality used in this experiment. The fit with the data can

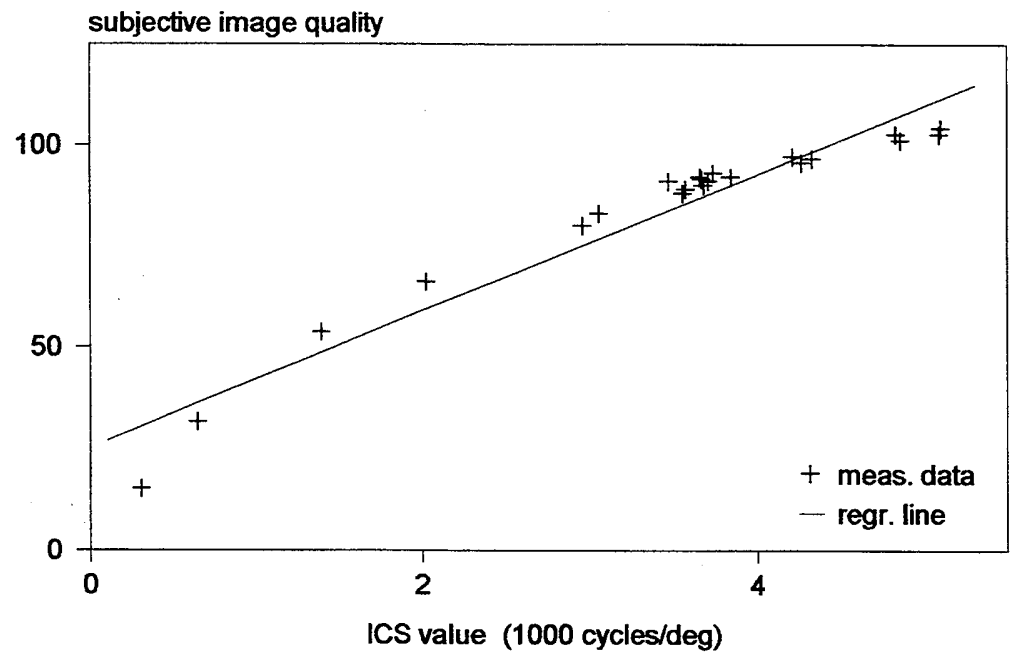

Figure 8.14: Measured subjective image quality as a function of ICS value for photographs reproduced with 22 different MTFs from an investigation by Higgins (1977). The solid line is the linear regression line. Correlation between measurements and calculations $93.3 \%$. 


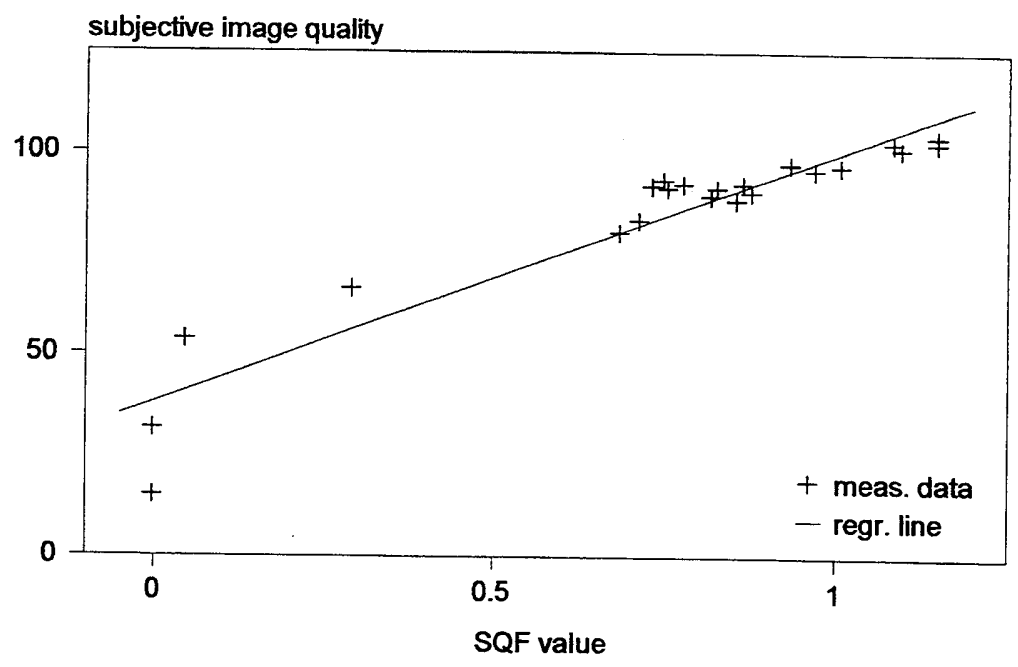

Figure 8.15: Measured subjective image quality as a function of SQF value for photographs reproduced with 22 different MTFs from an investigation by Higgins (1977). The solid line is the linear regression line. Correlation between measurements and calculations $89.3 \%$.

hardly be improved. The remaining spread is probably caused by a spread of the measurement data.

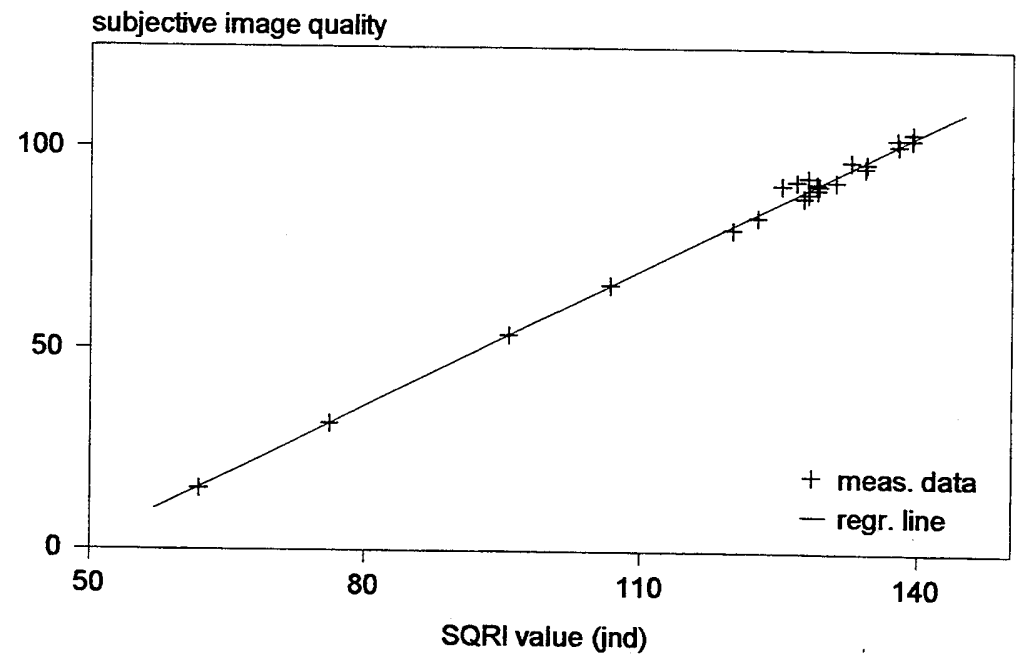

Figure 8.16: Measured subjective image quality as a function of SQRI value for photographs reproduced with 22 different MTFs from an investigation by Higgins (1977). The solid line is the regression line. Correlation between measurements and calculations $99.5 \%$. 


\subsection{Summary and conclusions}

In this chapter a model has been given for the nonlinear behaviour of the eye at suprathreshold levels of modulation. This model is important for a good description of perceived image quality, as the components of an image largely consist of modulations at suprathreshold level. According to this model, the perceived quality of an image is linearly related with the square root of the normalized modulation. This is based on the fact that image quality is linearly related with the number of discriminable levels, and that from the expressions for contrast discrimination given in the previous chapter follows that this number is linearly related with the square root of the normalized modulation. Furthermore, it has been shown that also the perceived contrast of sinusoidal luminance patterns is linearly proportional with the square root of the normalized modulation.

The perceived quality of an image is usually described with the aid of an image quality metric. In this chapter a survey has been given of various image quality metrics developed during the past decennia. From these metrics much can be learned about the contribution of different spatial frequencies to image quality. Also an image quality metric has been given that is based on the square root of the normalized modulation, according to the model given in the first part of this chapter. This metric is called SQRI or square-root integral.

In a principal test, the functional parameters of these metrics have been compared with the requirements for a good description of perceived image quality. For this analysis use has been made of experiments where the image quality was varied in steps of one just-noticeable difference. From the analysis, it appeared that the SQRI metric shows the best agreement with the requirements for a good description of image quality.

The metrics have also been tested by a comparison of the predicted image quality with the image quality obtained from subjective quality ratings for images of photographic pictures displayed with different MTFs. In this test, the SQRI showed a strictly linear correlation with the subjective measurements over a large range of image qualities.

\section{References}

Baldwin, M.W. (1940). The subjective sharpness of simulated television images. Bell System Technical Journal, 19, 563-587. 
Barten, P.G.J. (1987). The SQRI method: a new method for the evaluation of visible resolution on a display. Proceedings of the SID, 28, 253-262.

Barten, P.G.J. (1989). The square root integral (SQRI): a new metric to describe the effect of various display parameters on perceived image quality. Human Vision, Visual Processing, and Digital Display I, Proc. SPIE, 1077, 73-82.

Barten, P.G.J. (1990). Evaluation of subjective image quality with the square-root integral method. Journal of the Optical Society of America A, 7, 2024-2031.

Cannon, M.W. (1985). Perceived contrast in the fovea and the periphery. Journal of the Optical Society of America A, 2, 1760-1768.

Carlson, C.R. \& Cohen, R.W. (1972). Visibility of displayed information: image descriptors for displays. Report ONR-CR213-120-4F, Office of Naval Research, Arlington, VA.

Carlson, C.R \& Cohen, R.W. (1980). A simple psychophysical model for predicting the visibility of displayed information. Proceedings of the SID, 21, 229-246.

Charman, W.N. \& Olin, A. (1965). Image quality criteria for aerial camera systems. Photographic Science and Engineering, 9, 385-397.

Granger, E.M. \& Cupery, KN. (1972). An optical merit function (SQF), which correlates with subjective image judgments. Photographic Science and Engineering,
16, 221-230. Higgins, G.C. (1977). Image quality criteria. Journal of Applied Photographic Engineer
ing, 3, 53-60.

Legge, G.E. (1981) A power law for contrast discrimination. Vision Research, 21, 457-467.

van Meeteren, A. (1973). Visual aspects of image intensification. Ph. D. Thesis, University of Utrecht, Utrecht, The Netherlands.

Schade, O. (1951-1955). Image gradation, graininess, and sharpness in television and motion picture systems. Yournal of the SMPTE, 56, 137-171, 58, 181-222, 61, 97-164, and 64, 593-617.

Snyder, H.L. (1973). Image quality and observer performance. Perception of displayed information, L.M. Biberman, Editor, Chapter 3, Plenum Press, New York and
London.

Watanabe, A., Mori, T., Nagata S., and Hiwatashi, K (1968) Spatial sine-wave responses of the human visual system. Vision Research, 9, 1245-1263.

Watt, RJ. \& Morgan, M.J. (1983) The recognition and representation of edge blur: evidence for spatial primitives in human vision. Vision Research, 23, 14641477. 


\section{Chapter 9}

\section{Effect of various parameters on image quality}

\subsection{Introduction}

In the previous chapter various image quality metrics have been analyzed for the description of image quality. In these metrics, use was made of the MTF of the imaging system and the contrast sensitivity of the eye. From these metrics, the SQRI appeared to have the best relation with perceived image quality. The SQRI was originally developed for the evaluation of the effect of resolution on perceived image quality (Barten, 1987), but later, it appeared that it could also be used for the description of the effect of many other parameters on image quality (Barten, 1989a, 1990a). In this chapter the effect of a number of parameters on image quality will be treated. They will be analyzed with the aid of the SQRI and will largely be compared with published measurements. For the contrast sensitivity of the eye Eq. (3.26) in Chapter 3 will be used with the given typical values of the constants.

Beyond the contrast sensitivity of the eye, also the MTF of the imaging system plays an important role in the perception of image quality. Generally, some parameters influence the MTF, other parameters influence contrast sensitivity, and still other parameters influence both MTF and contrast sensitivity. In the SQRI the following parameters can be taken into account: Resolution is taken into account in the MTF. Addressability or addressable resolution is taken into account in the upper limit of the integration. Addressability is determined by the number of lines or the number of pixels with which the image is reproduced. Luminance, defined as the average luminance of the image, influences the contrast sensitivity and is therefore taken into account in the contrast sensitivity function. Contrast, defined by the size of the luminance variations divided by the average luminance, is taken into account in the MTF, because contrast changes the MTF with a certain factor. Gamma (see section 9.7) is also taken into account in the MTF, as it influences image quality in a similar way as contrast. Viewing distance is both taken into account in the contrast sensitivity function, because of its effect on field size, and in the MTF, because of its effect on angular spatial frequency. Image size at a constant viewing distance has the 
same effects as viewing distance and can therefore be treated in the same way. Noise in an image increases the modulation threshold and is therefore taken into account in the contrast sensitivity function. Luminance quantization used in digital images causes small local deviations from the original luminance and can therefore also be considered as a form of noise. Pixel geometry used in matrix displays influences the addressable resolution and can therefore be taken into account in the upper limit of
the integration.

All these effects will be treated in this chapter and the predicted results will mostly be compared with published measurements of image quality. The measurement results are usually obtained from the judgments by a panel of observers that observe the images under carefully standardized viewing conditions. For the judgment generally a category scaling method is used. In some cases, the subjects have to express their opinion in a scale number, and in other cases a standard series of verbal expressions is used, like "bad," "poor," "fair," "good," and "excellent." These expressions are then later transformed in a number. The results of the category scaling are often modified to transform them into a linear perceptual scale by using a technique based on Thurstone's law of categorical judgment (Togerson, 1958). By this technique, the scale is linearized with respect to equal subjective differences. This is comparable with the jnd scale used in the SQRI. In some measurements, two or more parameters are varied simultaneously. It appears that subjects still can give a consistent judgement of the total image quality. Under these conditions the visual system appears to weight the different parameters in a fixed relative proportion to each other. For these measurements the validity of a metric is of special importance, as it has to weight the different parameters in the same relative proportion as the visual
system.

For a comparison of the measured data with the calculated data, a linear regression will be made and the correlation coefficient $R^{2}$ will be calculated. This coefficient has the meaning that $1-R^{2}$ is the part of the variance of the results that has to be attributed to the sum of the inaccuracy of the experiment and the systematic deviation between theory and practice. The constants of the regression equation will be used to convert the calculated SQRI values in the image quality units used for the measurements. This enables an easy comparison between measurements and calculations. Both scale units will be used in the graphical representation of the results.

\subsection{Resolution and image size}

The effect of resolution on image quality was measured by Westerink \& Roufs (1989) together with the effect of image size in an investigation where both parameters were varied simultaneously. For this investigation projected colour-slides were 


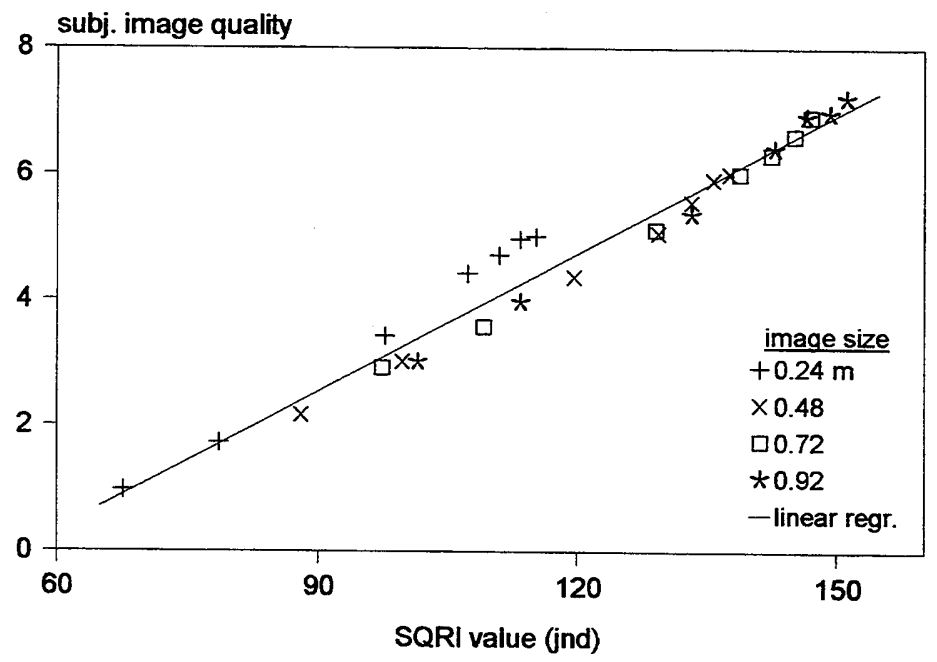

Figure 9.1: Linear regression between subjective image quality and SQRI value for measurements by Westerink \& Roufs (1989) with colour slides projected with different resolutions and sizes. Average luminance $30 \mathrm{~cd} / \mathrm{m}^{2}$. Viewing distance $2.9 \mathrm{~m}$. The SQRI values have been calculated with Eq. (8.5). The correlation between measurements and calculations is $96.3 \%$.

used with different resolutions and sizes. The images were square pictures of five different pictorial scenes. They were projected with an average luminance of $30 \mathrm{~cd} / \mathrm{m}^{2}$,

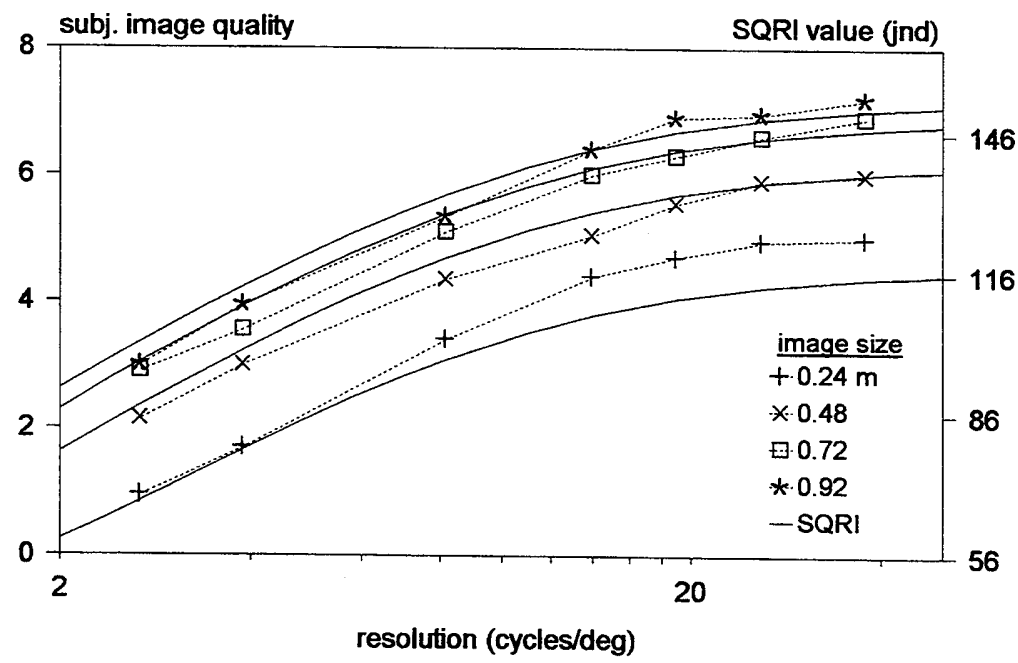

Figure 9.2: Measurement data of Fig. 9.1 plotted as a function of resolution with the image size as parameter. The resolution is expressed in the spatial frequency where the MTF has decreased with $50 \%$. The solid curves have been calculated with the SQRI with the aid of Eq. (8.5). 
and viewed from a distance of $2.9 \mathrm{~m}$. The size of the image was varied from $0.24 \mathrm{~m}$ to $0.92 \mathrm{~m}$, corresponding with a field size ranging from $4.7^{\circ}$ to $18^{\circ}$. The variation was obtained by using copies of the slides in four different sizes. The resolution was varied by defocusing the projector with the aid of a stepper motor. The MTF of the projector had a Gaussian shape. The resolution was expressed in the spatial frequency where the MTF has decreased with $50 \%$. The image quality was determined by using a 10-point numerical category scale. Twenty subjects took part in the experiment. The average rating was used for the results. Fig. 9.1 shows a linear regression between the measured and calculated data. The correlation is $96.3 \%$. In Fig. 9.2 the measurements and calculations are plotted as a function of resolution with the image size as parameter. In this figure, the resolution scale is expressed in the spatial frequency where the MTF has decreased to $50 \%$. The curves for the different image sizes are approximately parallel and show a saturation at a resolution of about 20 cycles/deg.

\subsection{Luminance and image size}

The effect of luminance on image quality together with the effect of image size was measured by Van der Zee \& Boesten (1980) of the same laboratory in an investigation where both parameters were varied simultaneously. For this investigation also projected colour-slides were used of which the luminance was varied with the aid of

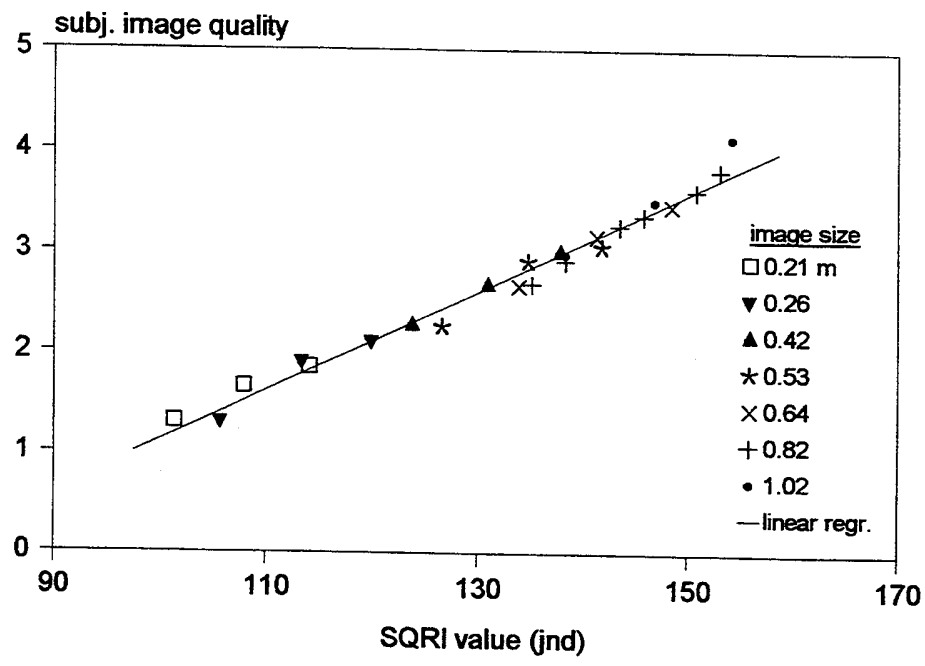

Figure 9.3: Linear regression between subjective image quality and SQRI value for measurements by van der Zee \& Boesten (1980) with colour slides projected with different luminance and sizes. Viewing distance $2.9 \mathrm{~m}$. The SQRI values have been calculated with Eq. (8.5). The correlation between measurements and calculations is $97.7 \%$. 


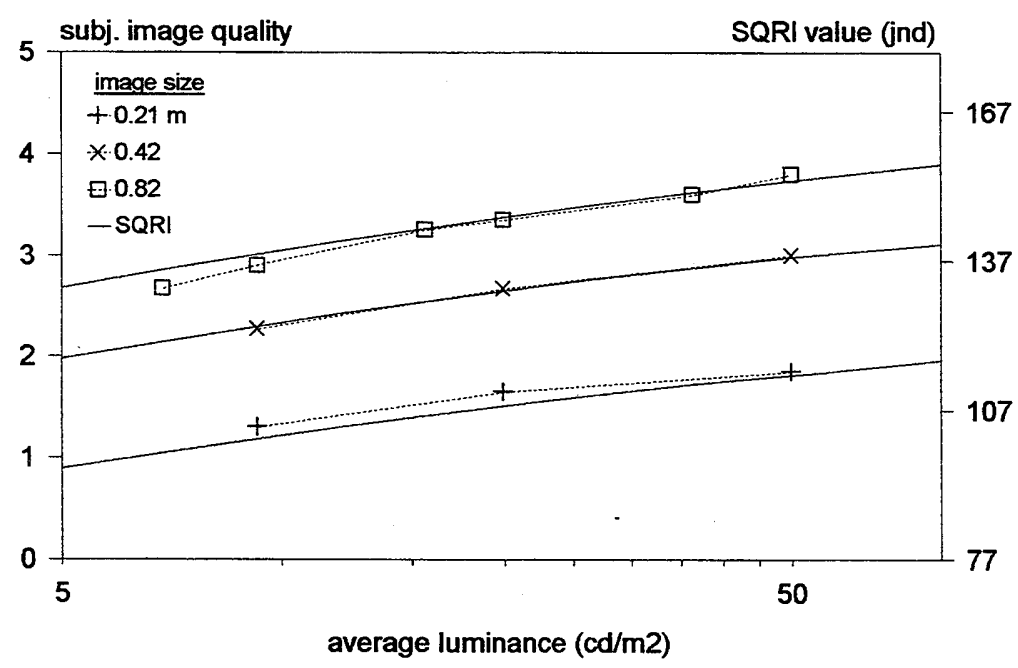

Figure 9.4: Measurement data of Fig. 9.3 plotted as a function luminance with image size as parameter. For clearness the results for only three image sizes are shown. The solid curves have been calculated with the SQRI with the aid of Eq. (8.5).

two projectors. For this investigation the same equipment with largely the same slides was used as was used later in the investigation by Westerink and Roufs treated in the previous section. Two images of the same scene - differing only in luminance and size - were projected simultaneously with the aid of the two projectors. The viewing distance was $2.9 \mathrm{~m}$. The projected image size was varied in seven steps from $0.21 \mathrm{~m}$ to $1.02 \mathrm{~m}$, corresponding with a field size ranging from $4.2^{\circ}$ to $20^{\circ}$. The luminance was varied with the aid of neutral density filters and was determined by measuring the open gate luminance. The average luminance may be assumed to be one tenth of the open gate luminance, similarly as in the experiments by Westerink and Roufs with the same slides. The perceived image quality was determined by using a 5-point category rating scale. Twenty-nine subjects took part in the experiments. The quality ratings were averaged over the 29 subjects. Fig. 9.3 shows a linear regression between measured and calculated data. The correlation is $97.7 \%$. In Fig. 9.4 the measurements and calculations are plotted as a function of luminance with the image size as parameter. For clearness only the results for three image sizes are shown. Curves for the other sizes are similar. The agreement between measurements and calculations is very good, certainly if it is considered that two parameters were varied simultaneously. The calculated curves are approximately parallel to each other. They show a saturation at a level of about $100 \mathrm{~cd} / \mathrm{m}^{2}$. 


\subsection{Anisotropic resolution}

Although the resolution of displayed images is usually equal in all directions, this need not always be so. If the resolution of an image is different in different directions, the two-dimensional extension given by Eqs. (8.8) or (8.9) can be used. This can be illustrated with measurements by Nijenhuis (1993) made on the same laboratory as where the experiments of the two previous sections were made. He measured the perceived image quality of a blurred image on a CRT monitor at several combinations of horizontal and vertical blur. The blur was electronically generated and was characterized by the standard deviation $\sigma$ of the Gaussian spread function used in the blurring process. The image was an artificial picture consisting of an evenly lit square area of $10 \mathrm{~cm} \times 10 \mathrm{~cm}$ with a luminance of $45 \mathrm{~cd} / \mathrm{m}^{2}$ on a background of $28 \mathrm{~cm} \times 28$ $\mathrm{cm}$ with a luminance of $10 \mathrm{~cd} / \mathrm{m}^{2}$. The average luminance of this image was 15.7 $\mathrm{cd} / \mathrm{m}^{2}$ and the viewing distance was $4 \mathrm{~m}$, which corresponds with a field size of $4^{\circ} \times 4^{\circ}$. For the evaluation of the results, the SQRI has been calculated for four directions: the horizontal direction, the vertical direction, and the two diagonal directions. The average of these four directions was used for the results. For the diagonal direction, it was assumed that the sigma of the blur can be derived from the sigma of the blur in horizontal and vertical direction by using the following equation:

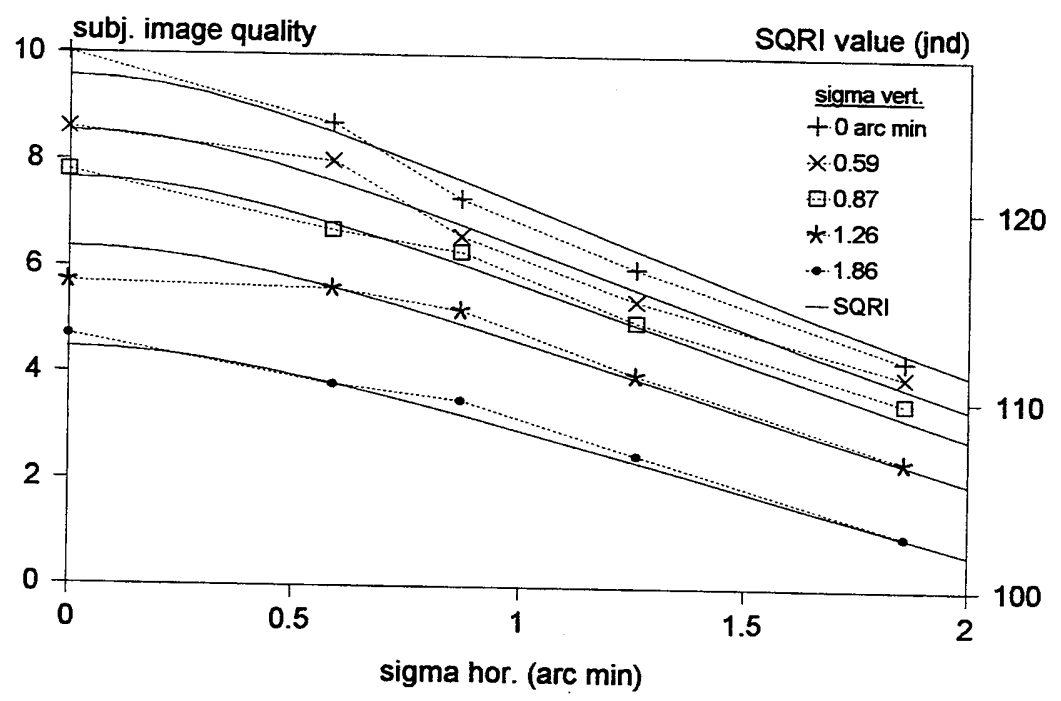

Figure 9.5: Subjective image quality of a blurred image measured by Nijenhuis (1993) as a function of the horizontal blur with the vertical blur as parameter. The blur is expressed in the standard deviation sigma of the spread function used in the blurring process. Average luminance $15.7 \mathrm{~cd} / \mathrm{m}^{2}$. Field size $4^{\circ} \times 4^{\circ}$. The solid curves have been calculated with the SQRI averaged over four directions, using Eqs. (8.5), (8.9), and (9.1). The correlation between measurements and calculations is $98.7 \%$. 


$$
\sigma_{\mathrm{dia}}=\sqrt{1 / 2 \sigma_{\mathrm{hor}}^{2}+1 / 2 \sigma_{\mathrm{ver}}^{2}}
$$

The measurement data and the calculation results are shown in Fig. 9.5 as a function of the horizontal blur with the vertical blur as parameter. The agreement between the measurements and calculations is very good. The correlation amounts to $98.7 \%$. From the figure, it can be seen that the curves of a constant vertical blur are not parallel but converge slightly at increasing horizontal blur. If the SQRI had been calculated by taking only the average over the horizontal and vertical directions, the curves had been parallel and the correlation between measurements and calculations would have been worse.

\subsection{Viewing distance, display size, and number of scan lines}

The perceived quality of an image is often influenced by the viewing distance. For the 625 lines PAL television system, the optimum viewing distance is assumed to be six times the picture height. For the 525 lines NTSC television system, this distance is assumed to be eight times the picture height. These values are based on practical experience and are confirmed by CCIR recommandations. At a given image size, a variation of the viewing distance means a variation of the field size. Furthermore, the angular resolution of the image is changed. Both factors have an opposite effect on image quality. This causes that there is an optimum distance where the image quality is maximum. The same holds for a chance in display size at a fixed viewing distance. This makes that there is also an optimum display size at a given viewing distance. The optimum viewing distance or the optimum image size of a television system can be calculated with the SQRI in the following way (Barten, 1990b):

For a television system, the resolution of the image is limited by the bandwidth of the system. This limitation can be taken into account in the SQRI in the upper limit of the integration. For the maximum spatial frequency of a television system holds

$$
u_{\max }=\frac{K N_{\mathrm{v}}}{2 h}
$$

where $N_{\mathrm{v}}$ is the visible number of scan lines, $h$ is the height of the image expressed in the angular size for the eye, and $K$ is the Kell factor. This is a factor by which the vertical resolution has to be reduced to avoid interference effects between the scan lines of the system and high spatial frequency components of the original image. For $K$ generally a value 0.7 is used. The bandwidth of the television signal is generally chosen such that the maximum spatial frequency in horizontal direction is equal to that in vertical direction. This means that the resolution is equal in both directions. 


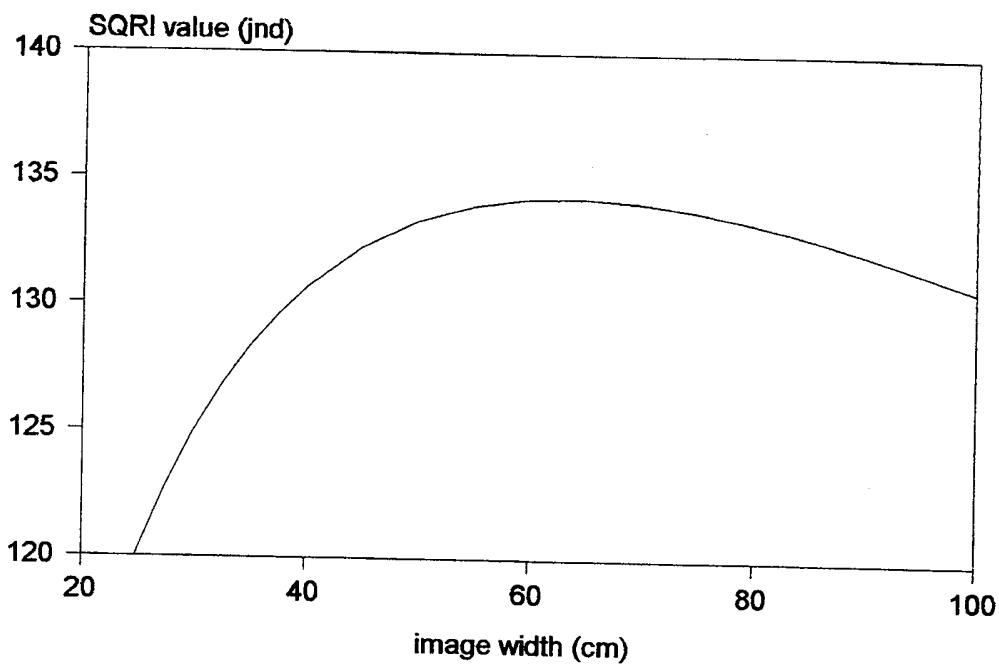

Figure 9.6: Perceived image quality as a function of image width, calculated with the $\mathrm{SQRI}$ for a PAL television picture viewed at a distance of $2.5 \mathrm{~m}$. The calculation has been made with the aid of Eqs. (8.5) and (9.2). A maximum occurs at a width of about $60 \mathrm{~cm}$. At this width, the viewing distance is about six times the image height.

The visible number of scan lines can be calculated from the total number of scan lines by subtracting the losses by vertical retracing (usually $8 \%$ ) and the losses by vertical overscan (usually 6\%). For the NTSC and PAL colour television systems furthermore a resolution loss of $29 \%$ and $26 \%$, respectively, has to be taken into account because of the presence of the colour sub-carrier. For a CRT display a Gaussian MTF may be assumed that is mainly influenced by the size of the electron spot. The width of this spot may be assumed to be about $0.4 \%$ of the picture width.

Under these conditions, the SQRI has been calculated for a PAL television picture with an average luminance of $100 \mathrm{~cd} / \mathrm{m}^{2}$ seen and a viewing distance of $2.5 \mathrm{~m}$. This calculation was made for various sizes of the image. The results are shown in Fig. 9.6 as a function of the image width. From the figure, it can be seen that the calculated curve shows indeed a maximum. At a lower size, the image quality decreases because of a decrease in field size, and at a higher size, the image quality decreases because of a decrease in angular resolution. The maximum occurs at an image width of about $60 \mathrm{~cm}$, which corresponds with an image height of about 45 $\mathrm{cm}$. At this size, the viewing distance is about six times the image height, which is considered as the optimum viewing distance for the PAL television system. 


\subsection{Contrast}

Contrast is defined here as the difference between two luminance values divided by the sum of them. According to this definition the maximum contrast is one. Stray light or reflected ambient light generally causes an equal increase of maximum and minimum luminance, whereas the difference remains the same. This gives a reduction of contrast. For the sinusoidal components of an image, this means, that the average luminance is increased, whereas the amplitude of the luminance variation has remained the same. This means that the modulation of each spatial frequency component of an image is multiplied with a factor $C$ given by

$$
C=\frac{L}{L+\Delta L}=\frac{1}{1+\frac{\Delta L}{L}}
$$

where $L$ is the average luminance without added light and $\Delta L$ is the amount of added light (Barten, 1989b). This factor is the same for all spatial frequencies. The reduction of the modulation with this factor also means that the MTF of the imaging system has to be multiplied by this factor. As the SQRI is proportional to the square root of the MTF, the SQRI has to be multiplied by the square root of $C$. So that

$$
J^{\prime}=\sqrt{C} J
$$

where $J$ is the SQRI value without contrast loss and $J^{\prime}$ is the SQRI value with contrast loss. A contrast loss of, for instance, $6 \%$ corresponding with $C=0.94$ would lead to a reduction of the SQRI value with $3 \%$, which is a substantial loss of image quality of about three jnds. The loss of image quality is often perceived as a loss of sharpness. This is caused by the multiplication of the MTF by the contrast factor, which appears to have the same effect on the perceived sharpness as a normal reduction of the MTF.

For most types of displays, the contrast loss by ambient light can be reduced by dark tinted screen glass. This goes, however, at the cost of the average luminance of the image. From one hand the image quality increases by the increase of the contrast, but from the other hand the image quality decreases by the decrease of the luminance. There is, however, an optimum glass transmission where the image quality reaches a maximum. This optimum can be calculated with the SQRI by using the above given expressions (Barten, 1991c).

For a reflective image, like a photograph, the contrast factor $C$ is generally equal to the reflectivity of the photographic material used for the print of the photograph. Here the amplitude of the luminance variations is multiplied by the reflectivity of the material, whereas the average luminance is usually determined by the luminance of the surrounding area.

An investigation where contrast loss in photographic images appeared to play 


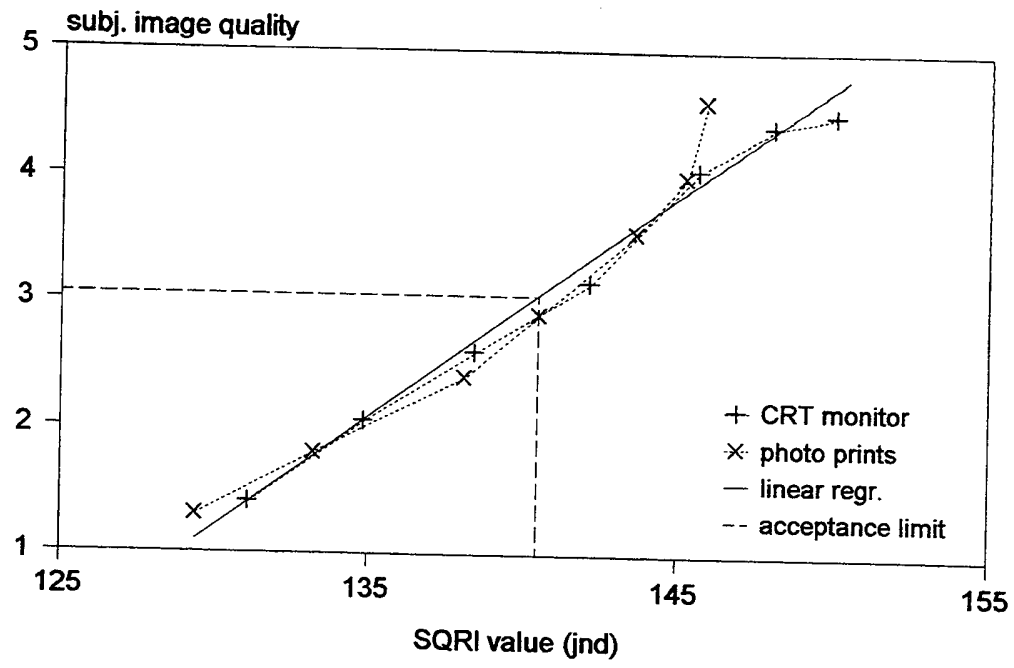

Figure 9.7: Linear regression between subjective image quality and SQRI value for measurements by Feng and Østberg $(1990,1991)$ with text images displayed on a CRT and on photo prints. Luminance $100 \mathrm{~cd} / \mathrm{m}^{2}$. Field size $23.7^{\circ} \times 31.3^{\circ}$. The dashed line represents the acceptance limit. The SQRI has been calculated with the aid of Eq. (8.5). In the calculation, it has been taken into account that the photo prints had a lower contrast. The correlation between measurements and calculations is $96.5 \%$ for the combined results of the two types of images.

an important role was made by Feng and Östberg (Feng, Östberg, and Lindström, 1990, Östberg \& Feng, 1991). They measured the perceived image quality of text images that were displayed with different focus conditions on a CRT together with images on photographic material that were copies of the same images. The luminance was $100 \mathrm{~cd} / \mathrm{m}^{2}$ and the viewing distance was $0.5 \mathrm{~m}$, corresponding with a field size of $23.7^{\circ} \times 31.3^{\circ}$. The photo prints were viewed under the same conditions as the CRT images, but the reflectivity of the material for the photographic prints was not $100 \%$ but $86 \%$ (Barten, 1992). Fig. 9.7 shows a linear regression between the subjective image quality and the calculated SQRI value for the results of both types of images. In the calculations, the contrast reduction of the photo prints was taken into account. The correlation between the measurements and the calculations for the combined results of the two types of images is $96.5 \%$. The dashed line represents the acceptance limit that the authors found in their investigation. In the figure no systematic difference can be seen between the two types of images. Östberg \& Feng (1991) found, however, unexplained systematic differences between the curves for the two types of images. However, they did not take the contrast loss of the photo prints into account and used, furthermore, the MTFA as a measure for image quality. If the contrast loss was not taken into account in the calculations for Fig. 9.7, the curve for the photo prints would have been shifted to the right over about nine jnds. 


\subsection{Gamma}

The relation between input and output luminance of an imaging system is not always linear. If it is nonlinear, it can often be described by an exponential relation given by

$$
L^{\prime}=\text { const. } L^{\gamma}
$$

where $L^{\prime}$ and $L$ are the output luminance and the input luminance, respectively, and $\gamma$ is the exponent of the exponential relation. The relation can also be written in the form

$$
\log L^{\prime}=\text { const. }+\gamma \log L
$$

From this equation follows, that if $L^{\prime}$ is plotted as a function of $L$ with a double logarithmic scale, a straight line is obtained with a slope equal to gamma.

For a gamma different from one, the modulation transfer from input to output cannot be described by an MTF, as the MTF concept is based on Fourier analysis that can only be applied to linear systems. With an exponential relation between the input and the output luminance, the output modulation depends on the luminance in the concerning part of the image. If gamma is higher than one, luminance variations increase in light parts of the image but decrease in dark parts. If gamma is lower than one, the inverse takes place. However, from a statistical analysis, it appears that the modulation increases linearly with gamma, if it is averaged over all luminance levels occurring in an image (Barten, 1996). This means that the average effect of gamma can be described by a multiplication of the MTF by gamma. As the SQRI is proportional with the square root of the MTF, the SQRI value and consequently also the image quality will increase linearly with the square root of gamma.

In practice, it appears that this is indeed so at low gamma values. However, at gamma values above an optimum of about 1.2 , the perceived image quality decreases again at a further increase of gamma. This decrease can be explained by a loss of discriminable details that disappear in the dark and light areas of an image. From measurements by van Hateren \& van der Schaaf (1996, Fig. 2), it appears that the luminance distribution of natural scenes can roughly be described by a rectangular function of the logarithm of the luminance. Fig. 9.8 shows a schematic representation of this distribution. From Eq. (9.6) follows that the width of this distribution on logarithmic scale increases proportionally with gamma. If gamma becomes higher than the optimum value, a part of the image is lost. From the probability density distribution shown in Fig. 9.8, it can be seen that the remaining part of the image is equal to $\gamma d \gamma$, if $\gamma_{0}$ is the optimum value of gamma. From experiments, it appears that for gamma values higher than $\gamma_{0}$, the perceived image quality decreases linearly with this remaining part, besides an increase with the square root of gamma due to the further increase of the modulation in this remaining part. The variation of the perceived image quality at a variation of gamma can, therefore, be described by the 


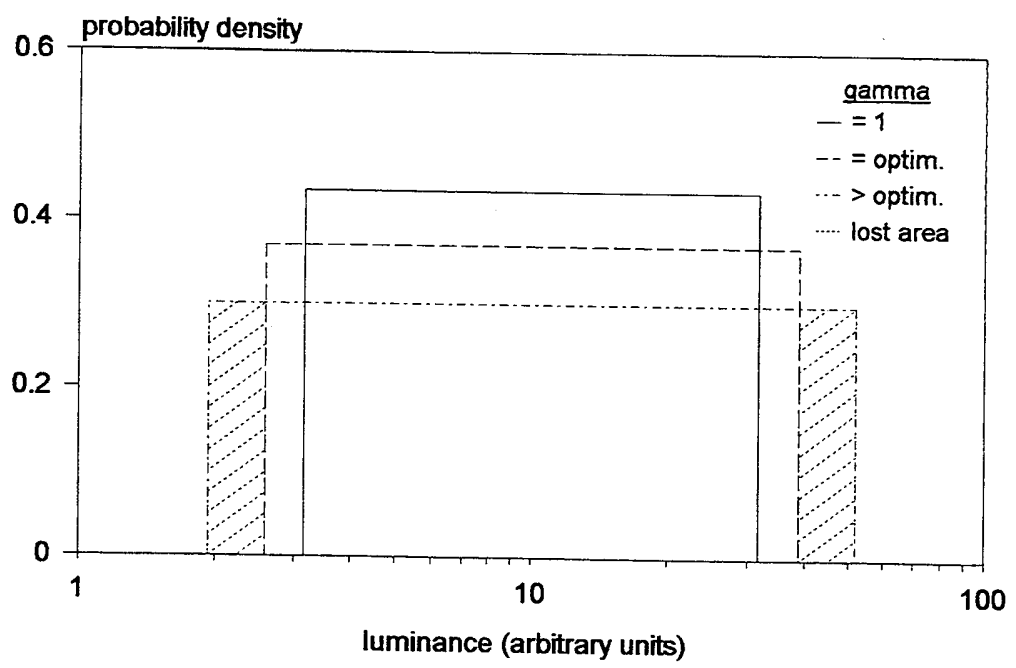

Figure 9.8: Schematic representation of the probability density distribution of the luminance in natural images and the change of this distribution at an increase of gamma. If gamma becomes higher than the optimum value, image details are lost in dark and light parts of the image.

following equations:

and

$$
J^{\prime}=\gamma^{0.5} J \quad \text { for } \gamma<\gamma_{0}
$$

$$
J^{\prime}=\gamma^{0.5}\left(\frac{\gamma_{0}}{\gamma}\right) J=\left(\frac{\gamma_{0}}{\gamma^{0.5}}\right) J \quad \text { for } \gamma \geq \gamma_{0}
$$

where $J$ is the SQRI value for $\gamma=1$, and $J^{\prime}$ is the SQRI value for other values of $\gamma$. According to this model given by the author (Barten, 1996), the image quality increases with the square root of gamma up to an optimum gamma value and decreases again at higher gamma values inversely with the square root of gamma.

Fig. 9.9 shows an evaluation of measurements by Roufs et al. (1994) with the aid of Eqs. (9.7) and (9.8). They measured the subjectively perceived image quality as a function of gamma over a large range of gamma values using three natural images: a town hall, a terrace scene, and a woman's portrait. The original images were colour photographs taken with accompanying luminance bars for a calibration of the gamma of the total system. The images were displayed on a video monitor of which the gamma could be electronically varied, while keeping the average luminance constant. The luminance was about $25 \mathrm{~cd} / \mathrm{m}^{2}$. The size of the pictures was $28 \mathrm{~cm} \times$ $28 \mathrm{~cm}$ and they were viewed at a distance of $2.1 \mathrm{~m}$, corresponding with a field size of $6.8^{\circ} \times 6.8^{\circ}$. The ratings were made with a 10-point numerical scale. The measurement data are the averages of the ratings by four observers. The optimum gamma was 1.35. 


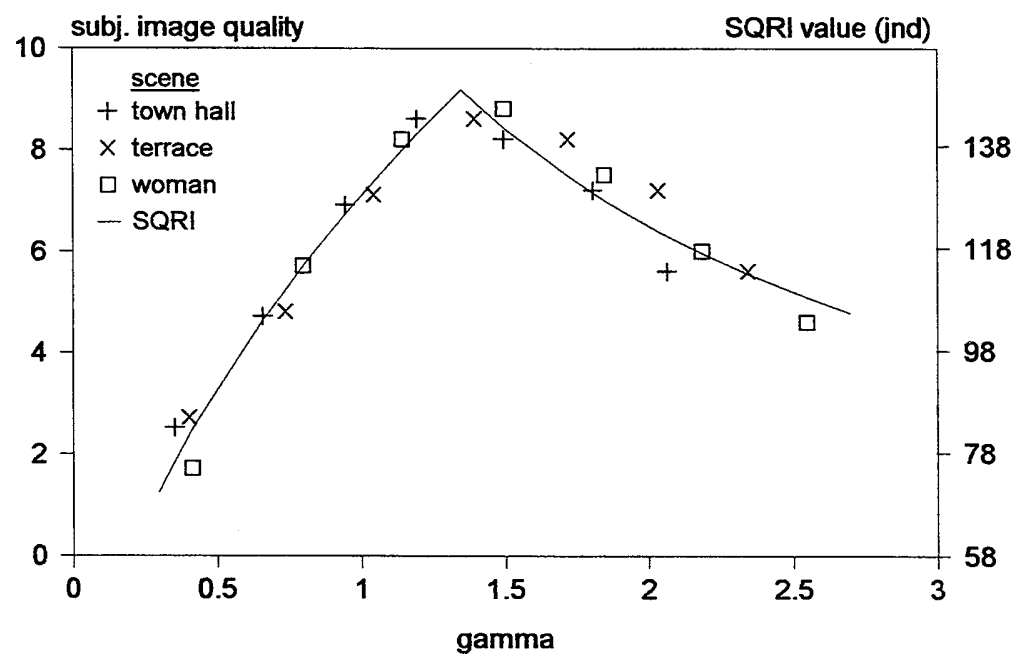

Figure 9.9: Subjective image quality as a function of gamma measured by Roufs et al. (1994) for three natural images displayed on a CRT monitor. Luminance $25 \mathrm{~cd} / \mathrm{m}^{2}$. Field size $6.8^{\circ} \times 6.8^{\circ}$. The curve through the data has been calculated with the SQRI with the aid of Eq.( 8.5), using Eqs. (9.7) and (9.8) for the effect of gamma. Optimum gamma 1.35. The correlation between measurements and calculations $95.3 \%$.

The correlation between the measurements and calculations is $95.3 \%$.

Fig. 9.10 shows a similar evaluation of measurements by Shimodaira et al. (1995). They measured the perceived image quality at ten different gamma values for five digital standard test charts of the ITEJ (Institute of Television Engineers of Japan). The images were displayed on an 8.6-inch TFT-LCD (thin film transistor liquid crystal display) and viewed at a distance of six times the screen height, which corresponds with a field size of $12.7^{\circ} \times 9.5^{\circ}$. The average luminance was about 14 $\mathrm{cd} / \mathrm{m}^{2}$. The image quality judgments were made with a 5-point rating scale. The given data are the averages of the ratings by 15 observers. The optimum gamma was 1.13. The optimum gamma for each of the separate pictures differed slightly from each other. This causes a rounding of the maximum for the average of the pictures. The correlation between the measurements and calculations is $98.2 \%$.

Fig. 9.11 shows similar results for measurements by Mitsubayashi et al. (1996) with largely the same team of authors as at for the previous investigation. They measured the perceived image quality at ten different gamma values for five digital standard test charts of the ITEJ (Institute of Television Engineers of Japan). The images were now displayed on a 14-inch CRT monitor used at three different luminance levels: 6,14 , and $40 \mathrm{~cd} / \mathrm{m}^{2}$. As the results for these luminance levels did not differ much from each other, only the data for $14 \mathrm{~cd} / \mathrm{m}^{2}$ were used for the evaluation given here. The images were viewed at a distance of six times the screen 


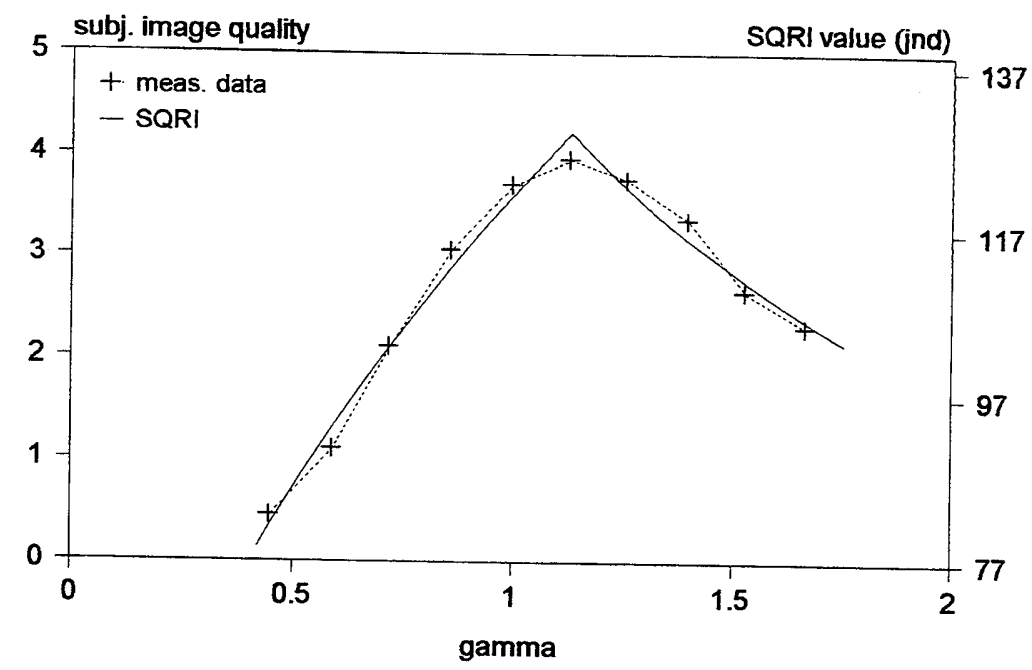

Figure 9.10: Subjective image qualityas a function of gamma measured by Shimodaira et al. (1995) for the average of five test images displayed on a TFT-LCD. Luminance 14 $\mathrm{cd} / \mathrm{m}^{2}$. Field size $12.7^{\circ} \times 9.5^{\circ}$. The curve through the data has been calculated with the SQRI with the aid of Eq. (8.5), using Eqs. (9.7) and (9.8) for the effect of gamma. Optimum gamma 1.13. The correlation between measurements and calculations is $98.2 \%$.

height, which corresponds with a field size of $12.7^{\circ} \times 9.5^{\circ}$. The image quality judg-

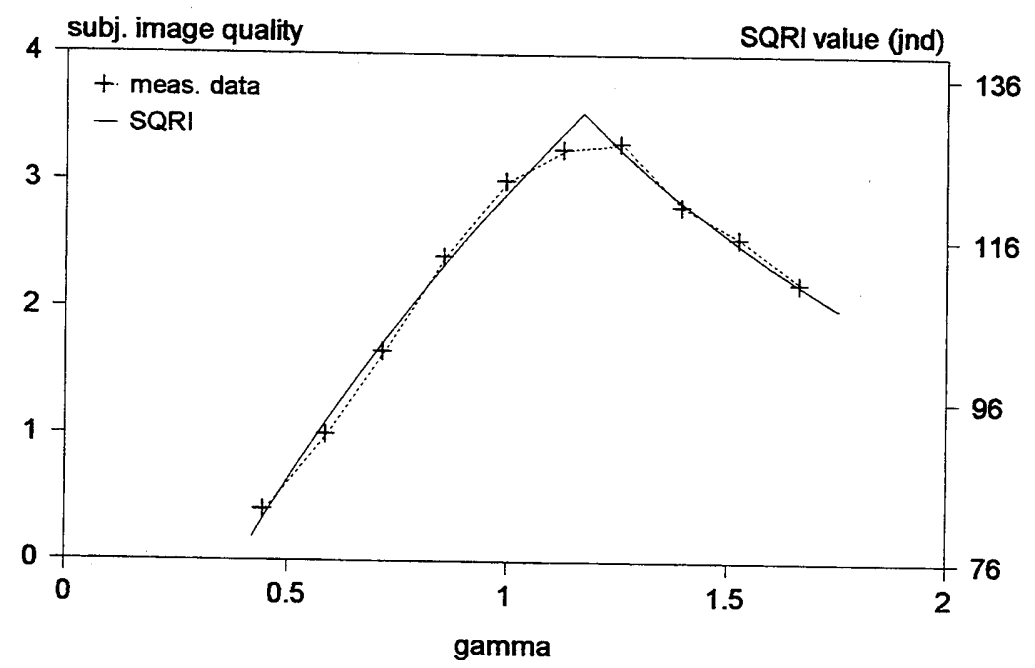

Figure 9.11: Subjective image quality as a function of gamma measured by Mitsubayashi et al. (1996) for the average of five test images displayed on a CRT monitor. Luminance $14 \mathrm{~cd} / \mathrm{m}^{2}$. Field size $12.7^{\circ} \times 9.5^{\circ}$. The curve through the data has been calculated with the SQRI with the aid of Eq. (8.5), using Eqs. (9.7) and (9.8) for the effect of gamma. Optimum gamma 1.17. The correlation between measurements and calculations is $99.3 \%$. 
ments were made with a 5-point scale. The data were averaged over observers and images. The optimum gamma value was 1.17. The correlation between the measurements and calculations is $99.3 \%$. From Figs. 9.9 through 9.11 , it can be seen that the used model for the effect of gamma on perceived image quality agrees very well with the measurements.

\subsection{Noise}

From the experience obtained with the SQRI, it appears that the effect of noise on image quality can best be described by the increase of the modulation threshold due to the presence of noise (Barten, 1991a). This increase can be calculated with the aid of Eqs. (2.50) and (2.43) given in Chapter 2. These expressions will further be used here for the analysis of the effect of noise on image quality given by the examples of the following measurements.

Besides the image quality measurements of photographic images with different MTFs described in section 8.6 of the previous chapter, Higgins (1977) also made measurements with different amounts of noise. These measurements were made for three resolution levels by using three different MTFs in the photographic reproduc-

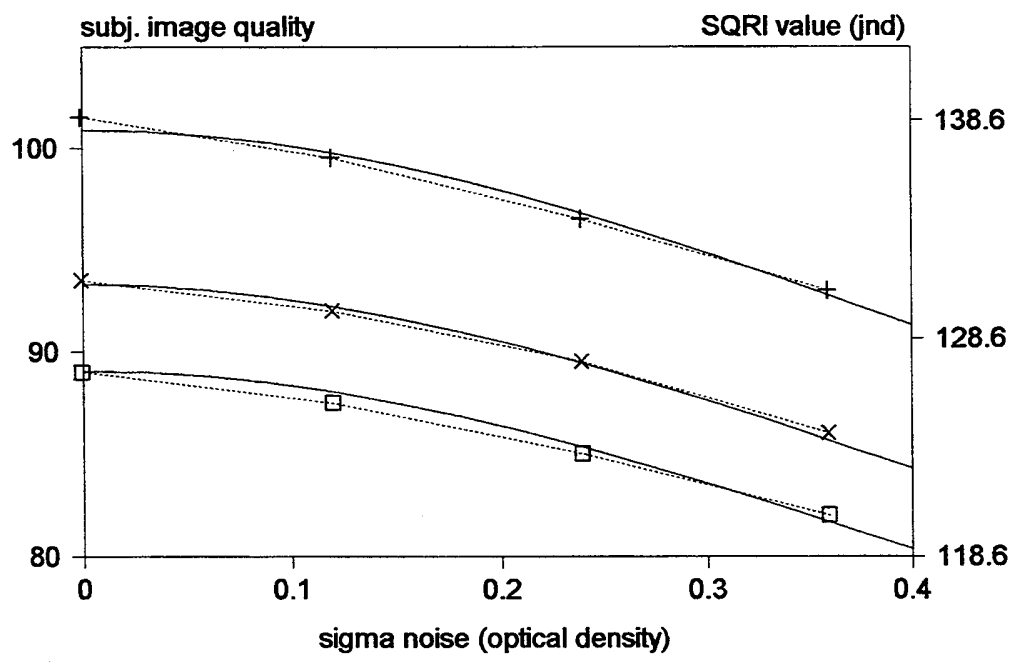

Figure 9.12: Subjective image quality measured by Higgins (1977) as a function of the sigma of the noise for photographic images with three different resolution levels. The sigma of the noise is expressed in units of optical density. The solid curves have been calculated with the SQRI with the aid of Eq. (8.5), using Eqs. (2.50), (2.43) and (2.41) for the dependence on noise. The correlation between measurements and calculations is $99.7 \%$. 
tion process before adding the noise. The further conditions were the same as for the investigation described in section 8.6. By the simultaneous variation of noise and resolution a good impression can be obtained of the relative weight of these parameters on image quality. Measurements and calculations are shown in Fig. 9.12. In this experiment the sigma of the noise was expressed in units of optical density. The correlation between measurements and calculations is $99.7 \%$. The good agreement between measurements and calculations confirms the validity of the metric used for the evaluation of image quality.

Kayargadde (1995) made a study in which different aspects of noise were investigated. He did not measure the image quality in the presence of noise, but the perceived noisiness. However, the inverse of the noisiness can be considered as a measure for image quality. The reported scaled noisiness is, therefore, compared here with the calculated SQRI value after adding a negative sign to the data, which is shown in the figures as negatively scaled noisiness. Furthermore, the original data of the sigma of the noise are transformed in relative units with respect to the average luminance. This relative sigma is here shortly called sigma noise.

In one experiment noise was added to a pure white field displayed on a CRT monitor. The images had a size of $0.17 \mathrm{~m} \times 0.17 \mathrm{~m}$ and were viewed from a distance of $1.4 \mathrm{~m}$ corresponding with a field size of $7^{\circ} \times 7^{\circ}$. Two different luminance levels were used: $20 \mathrm{~cd} / \mathrm{m}^{2}$ and $30 \mathrm{~cd} / \mathrm{m}^{2}$. Subjects were asked to judge the noisiness of the

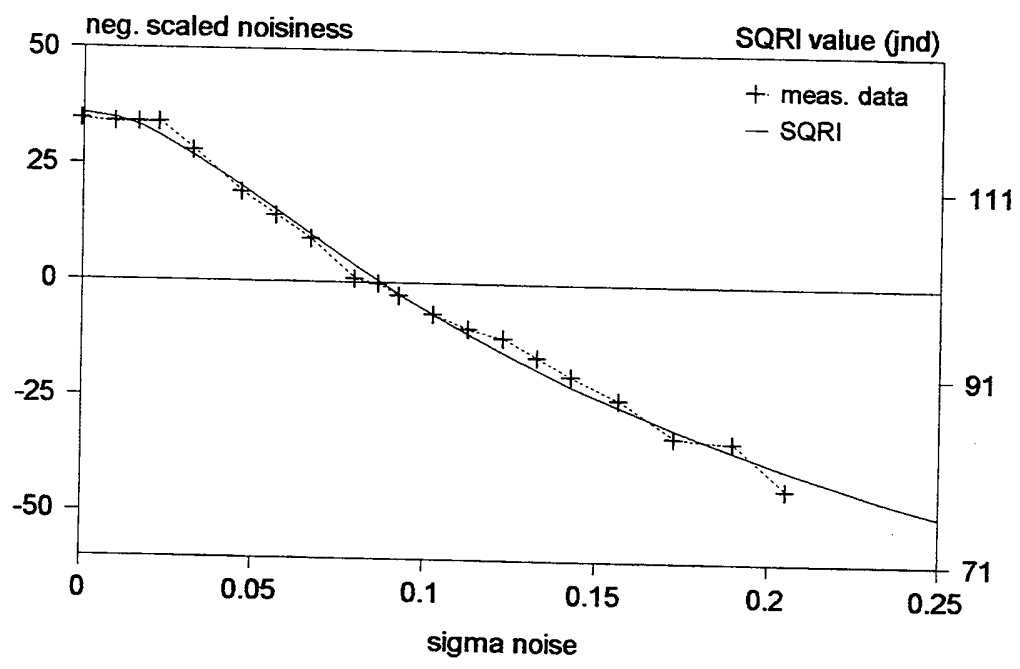

Figure 9.13: Negatively scaled noisiness as a function of the sigma of the noise measured by Kayargadde (1995) for a white field on a CRT monitor. Luminance $30 \mathrm{~cd} / \mathrm{m}^{2}$. Field size $7^{\circ} \times 7^{\circ}$. The solid curve has been calculated with the SQRI with the aid of Eq. (8.5), using Eqs. (2.50), (2.43) and (2.41) for the dependence on noise. The correlation between measurements and calculations is $99.4 \%$. 
picture. The data are the average results from eight subjects. Fig. 9.13 shows the so obtained data with a negative sign as a function of the sigma of the noise for the luminance level of $30 \mathrm{~cd} / \mathrm{m}^{2}$. The results for $20 \mathrm{~cd} / \mathrm{m}^{2}$ are similar. The curve through the data has been calculated with the SQRI. The correlation between the measurements and calculations is $99.4 \%$. The agreement between measurements and calculations is somewhat remarkable, as the image just consists of an evenly lit white field. In this field no pictorial information is present that could be masked by the noise. The observed noisiness is obviously perceived as a degrading of the flat image.

It is well known that noise in a dark image is more annoying than noise in a light image. One would therefore expect that noise in a dark part of an image is also more annoying than noise in a light part. As far as the total image is darker or lighter, the effect on image quality is taken into account in the sigma of the noise, as this is expressed in relative units. This relative sigma of the noise is equal to the absolute sigma of the noise expressed in luminance units divided by the average luminance. Within an image, the relative sigma is the same for the dark and light parts, because the average luminance of the total image is the same for both areas. The question rises if this is the right way to treat the effect of noise on image quality. Kayargadde analyzed this aspect of noise by measuring the perceived noisiness as a function of the local luminance in a part of the image, while keeping the average luminance in the image constant. The image consisted of nine square blocks with five different

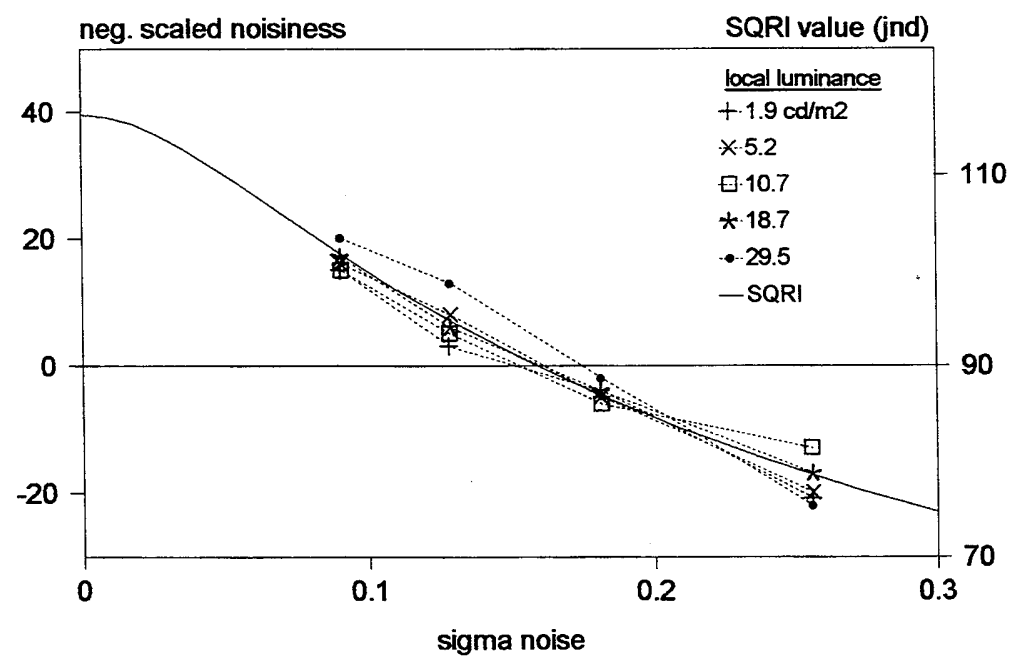

Figure 9.14: Negatively scaled noisiness as a function of the sigma of the noise measured by Kayargadde (1995) for the central part of an image consisting of blocks with different luminance. The local luminance of the central block was varied. Average luminance of the total image $12.8 \mathrm{~cd} / \mathrm{m}^{2}$. Field size $8.2^{\circ} \times 82^{\circ}$. The solid curve has been calculated with the SQRI with the aid of Eq. (8.5), using Eqs. (2.50), (2.43) and (2.41) for the dependence on noise. The correlation between measurements and calculations is $95.9 \%$. The measurement data show no systematic dependence on local luminance. 
luminance levels ranging from 1.9 to $29.5 \mathrm{~cd} / \mathrm{m}^{2}$. The average luminance was 12.8 $\mathrm{cd} / \mathrm{m}^{2}$. The total size of the image was $0.2 \mathrm{~m} \times 0.2 \mathrm{~m}$ and the viewing distance was $1.4 \mathrm{~m}$, corresponding with a field size of $8.2^{\circ} \times 8.2^{\circ}$. The noise was added only in the central block of the image. Subjects were asked to judge the noisiness in this part. The luminance of this block was changed to each of the five mentioned luminance levels by exchanging the luminance of this block with one other block of the image. The measurement data are the average results from eight subjects. Fig. 9.14 shows the so obtained data as a function of the (relative) sigma of the noise with the local luminance as parameter. The solid curve through the data has been calculated with the SQRI. For the calculation of the relative sigma of the noise used in the SQRI, the average luminance of the total image was used. The correlation between the measurements and calculations is $95.9 \%$. The measured data show no systematic difference between the different local luminance levels, although these differ up to a factor 15 from each other. Only the results for the highest luminance level seem to differ systematically from the other luminance levels, but this difference inverses at the highest noise level. From the results of this experiment, it may be concluded that only the average luminance of an image plays a role for the effect of noise on image quality, even if the different parts of an image differ considerably in luminance.

The luminance deviations caused by noise usually show a Gaussian distribution. However, this distribution needs not always to be Gaussian. Kayargadde

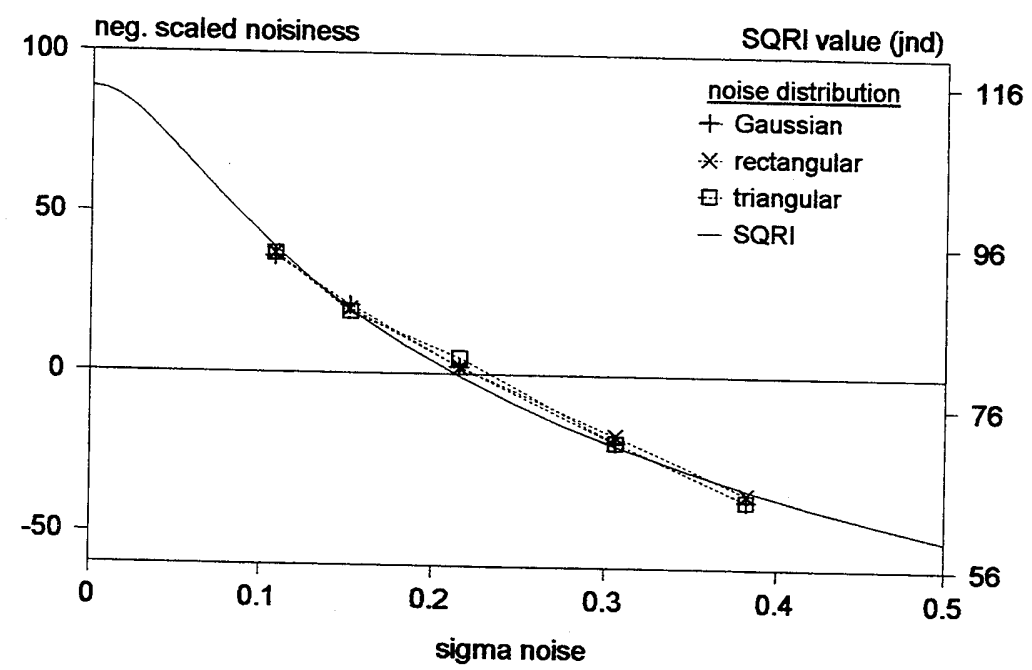

Figure 9.15: Negatively scaled noisiness as a function of the sigma of the noise measured by Kayargadde (1995) for a white field with three different noise distributions. Luminance $20 \mathrm{~cd} / \mathrm{m}^{2}$. Field size $7^{\circ} \times 7^{\circ}$. The solid curve has been calculated with the SQRI with the aid of Eq. (8.5), using Eqs. (2.50), (2.43) and (2.41) for the dependence on noise. The correlation between measurements and calculations is $99.4 \%$. There is no systematic difference between the different noise distributions. 
investigated the effect of different distributions of the luminance deviations of the noise on the perceived noisiness. Besides the Gaussian distribution, he also used a rectangular and a triangular distribution. The amount of noise was in all these situations characterized by the standard deviation of these distributions. Also in the SQRI the sigma of the noise is determined by the standard deviation of the luminance deviations, as follows from Eq. (2.31) given in Chapter 2. The noise was added to a white field with a luminance of $20 \mathrm{~cd} / \mathrm{m}^{2}$. The size of the image was $0.17 \mathrm{~m} \times$ $0.17 \mathrm{~m}$ and the viewing distance was $1.4 \mathrm{~m}$, corresponding with a field size of $7^{\circ} \times 7^{\circ}$. Subjects were asked to judge the noisiness of the image. The measurement data are the average results from eight subjects. Fig. 9.15 shows the so obtained data for the three different distributions as a function of the sigma of the noise. The solid curve through the data has been calculated with the SQRI. The correlation between the measurements and calculations is $99.4 \%$. The measured data show no systematic difference for the different distributions. This means that for the calculation of the effects of noise, only the sigma derived from the standard deviation of the luminance deviations is important, regardless the type of distribution.

Kayargadde also measured the effect of noise on natural images: a terrace scene and a woman's portrait. The size of the image was $0.24 \mathrm{~m} \times 0.24 \mathrm{~m}$ and the viewing distance was $1.4 \mathrm{~m}$, corresponding with a field size of $9.8^{\circ} \times 9.8^{\circ}$. Subjects were asked to judge the noisiness of the image. The measured data are the average results from eight subjects. Fig. 9.16 shows the so obtained data as a function of the sigma of the

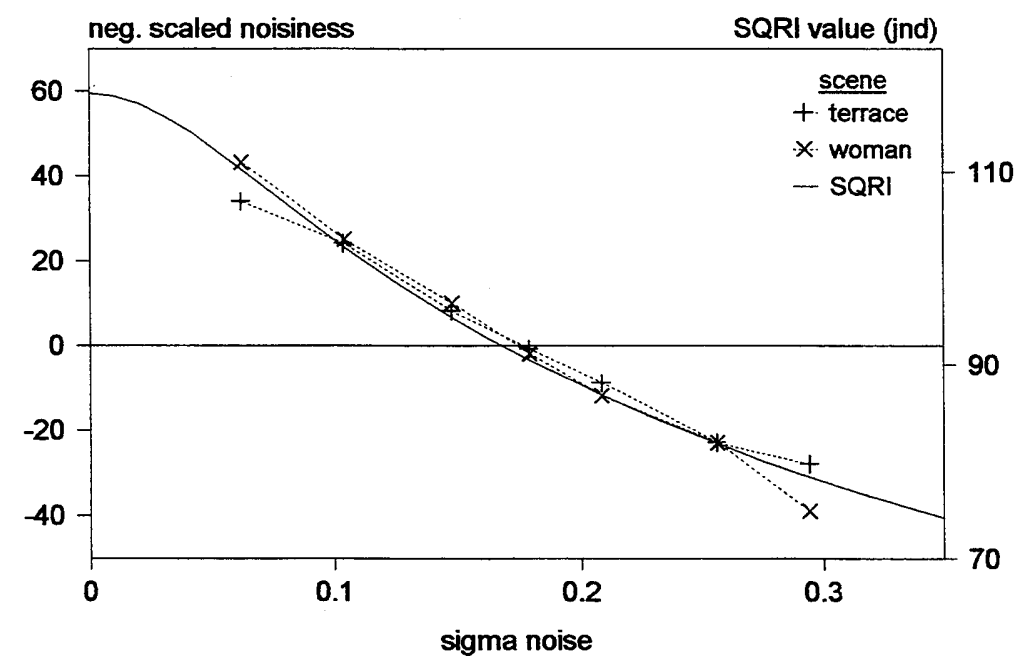

Figure 9.16: Negatively scaled noisiness as a function of the sigma of the noise measured by Kayargadde (1995) for two natural scenes. Luminance $9 \mathrm{~cd} / \mathrm{m}^{2}$. Field size $9.8^{\circ} \times 9.8^{\circ}$. The solid curve has been calculated with the SQRI with the aid of Eq. (8.5), using Eqs. (2.50), (2.43) and (2.41) for the dependence on noise. The correlation between measurements and calculations is $97.9 \%$. 
noise. The solid curve has been calculated with the SQRI. As expected, the results for both pictures do not show a systematic difference. The correlation between the measurements and calculations is $97.9 \%$ for the combined results of both pictures.

\subsection{Pixel density and luminance quantization}

Nowadays images are often stored and transferred in digital form. For the conversion in digital format, the images are spatially sampled and the luminance information is quantified. Also at the reproduction of an image on a matrix display, like a LCD (liquid crystal display), ELD (electro luminescence display) or PDP (plasma display panel), the information is spatially sampled. Spatial sampling sets a limit to the obtainable resolution and luminance quantization causes local deviations from the original luminance.

In the SQRI, the resolution limit caused by spatial sampling can be taken into account in the upper limit of the integration. The resolution limit is given by the Nyquist frequency of the pixel structure

$$
u_{\max }=u_{\mathrm{N}}=\frac{1}{2 p}
$$

where $u_{\mathrm{N}}$ is the Nyquist frequency and $p$ is the centre-to-centre distance of the pixels expressed in angular size for the eye. However, sampling does not only influence the integration limit, but also the part of the MTF below this limit. The MTF below this limit can be described by the following sinc function (Barten, 1991b):

$$
M(u)=\left|\frac{\sin (\pi p u)}{\pi p u}\right|
$$

These expressions are valid for a monochrome display. For a colour display, $p$ has to be multiplied with a certain factor to replace $p$ by the average sampling distance of the three colours.

The luminance deviations caused by the luminance quantization can be considered as a form of noise (Barten, 1993). The luminance distribution of this noise is in principle rectangular over the quantization distance $\Delta L$ between two luminance levels. As the measurements by Kayargadde given in the previous section have shown, this type of distribution of the luminance deviations is completely comparable with the usual Gaussian distribution, if the standard deviation of the distribution is used to characterize the noise. For the so obtained standard deviation of this noise can be derived (Barten, 1993)

$$
\sigma_{n}=\frac{1}{\sqrt{12}} \frac{\Delta L}{L}
$$


where $L$ is the average luminance. The luminance levels used for the quantization are mostly equally spaced between zero and maximum luminance. For this situation

$$
\Delta L=\frac{L_{\max }}{N-1}
$$

where $L_{\max }$ is the maximum luminance and $N$ is the number of luminance levels. From the last two equations follows

$$
\sigma_{n}=\frac{1}{\sqrt{12}} \frac{L_{\max } / L}{N-1}
$$

In practice the ratio $L_{\max } / L$ in this expression can vary from about 1.25 for text images of black letters on a white background to 10 or more for natural images.

The spectral noise density can be calculated from $\sigma_{n}$ with the aid of Eq. (2.41) given in Chapter 2:

$$
\Phi_{\mathrm{n}}=\frac{\sigma_{\mathrm{n}}{ }^{2}}{2 u_{\text {max }} 2 v_{\text {max }}}
$$

where $u_{n \max }$ and $v_{\text {nmax }}$ are the maximum spatial frequencies of the noise in $x$ and $y$ directions. These spatial frequencies are much higher than the maximum spatial frequencies $u_{\max }$ and $v_{\max }$ that can be derived from the pixel size $p$ over which the luminance level is digitized. The truncation that takes place in the digitizing process creates higher harmonics of these frequencies. These frequencies are not visible in the displayed image but have to be taken into account in the calculation of the spectral noise density. From a mathematical analysis of this process can be found that in practice approximately the following relations hold (Barten, 1993):

$$
u_{\text {mmax }}=3 u_{\max } \text { and } v_{\text {max }}=3 v_{\max }
$$

where $u_{\mathrm{nmax}}$ and $v_{\mathrm{n} \max }$ are given by $\mathrm{l} /(2 p)$. From these equations follows for the spectral noise density caused by the luminance quantization

$$
\Phi_{\mathrm{n}}=\frac{\sigma_{\mathrm{n}}^{2} p^{2}}{3^{2}}
$$

With these equations the SQRI has been calculated for an experiment by Silverstein et al. (1990) where the subjective image quality was measured as a function of the number of quantization levels. The images were graphical images with different pixel geometries and different combinations of colours generated on a high resolution colour CRT to simulate the effects that would appear on a matrix display. For this type of pictures a factor three may be assumed for the ratio between the maximum luminance and the average luminance. The viewing distance was $0.81 \mathrm{~m}$ and the field size was $1.5^{\circ} \times 1.5^{\circ}$. The simulated pixel density $1 / p$ was 55 pixels $/ \mathrm{cm}$, so that $p$ was 0.77 arc min. Ten subjects took part in the experiment. The measurement data are averaged over subjects, image types, colours, and two pixel geometries: 


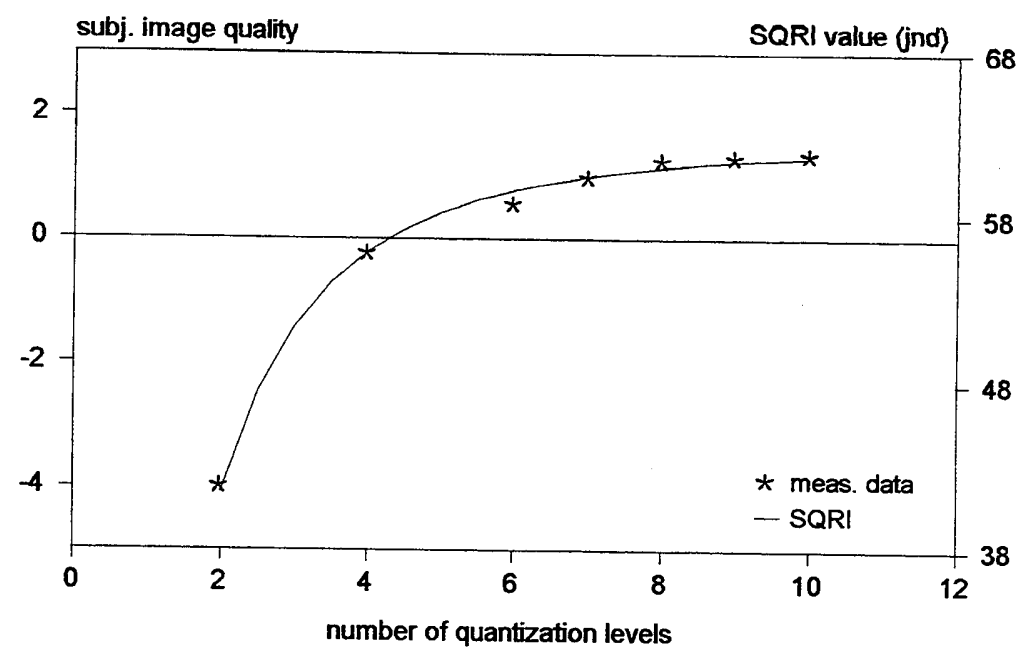

Figure 9.17: Subjective image quality as a function of the number of quantization levels measured by Silverstein et al. (1990) for graphical images simulated on a high resolution colour CRT. Field size $1.5^{\circ} \times 1.5^{\circ}$. The curve through the data has been calculated with the SQRI. The correlation between measurements and calculations is $99.7 \%$. The image quality saturates at a number of about eight quantization levels.

triad and quad. To take the geometric effect of these colour configurations into account, the value of $p$ in Eqs. (9.9) and (9.10) had to be multiplied with a factor 1.83 to obtain the average sampling distance for the three colours. Measurement data and calculation results are shown in Fig. 9.17. The correlation between the measure ments and calculations is $99.7 \%$. From the figure, it appears that the dependence of the image quality on the number of quantization levels is well described by the given model. It is further almost surprising to see that the image quality already saturates at about eight quantization levels (three bits).

In the experiment shown in Fig. 9.17, the pixel density $1 / p$ was constant. In another expeximent made by the same investigators (Kranz \& Silverstein, 1990) the pixel density was varied besides the number of quantization levels. Twelve subjects took part in this experiment. Other conditions were the same as in the previous experiment. Measurements and calculations are shown in Fig. 9.18. The number of quantization levels is expressed here in bits. The measurement data are again averaged over subjects, image types, colours, and two pixel geometries: triad and quad. The correlation between the measurements and calculations is $96.6 \%$. The figure shows a good agreement between measurements and calculations.

In the design of an electronic imaging system, bits of luminance levels can often be exchanged with pixel density. For an optimum design, a good knowledge of the effects of both parameters on image quality is important. The analysis given here 


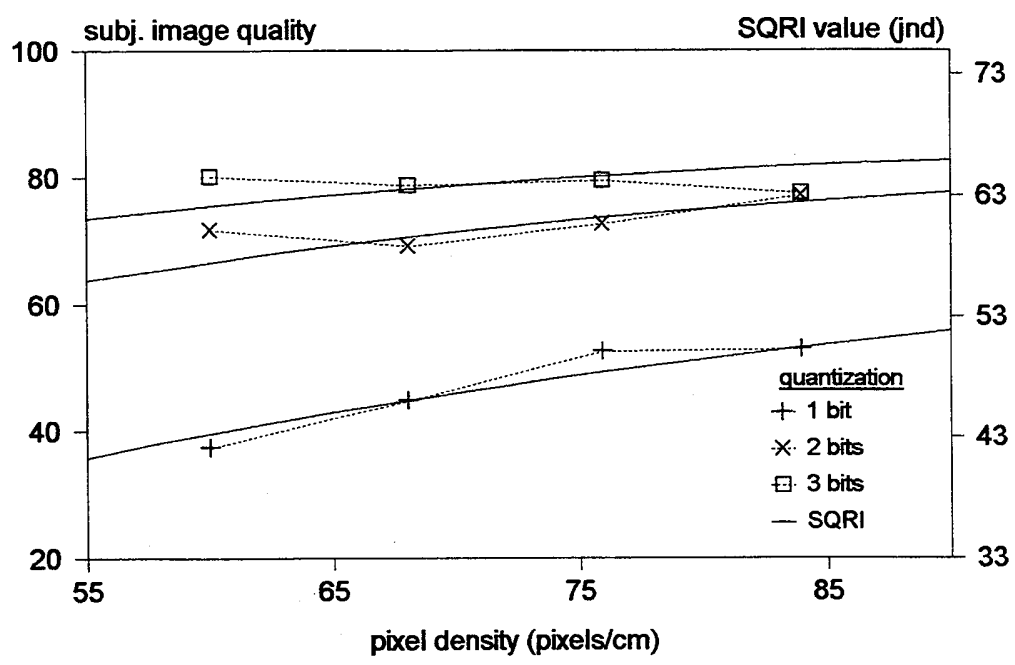

Figure 9.18: Subjective image quality as a function of pixel density measured by Kranz \& Silverstein (1990) for graphical images simulated on a high resolution colour CRT with three different numbers of quantization levels. The number of quantization levels is expressed in bits. Field size $1.5^{\circ} \times 1.5^{\circ}$. The solid curves have been calculated with the SQRI. The correlation between measurements and calculations is $96.6 \%$.

can be used to find an optimum tradeoff between pixel density and bits of luminance levels. It must, however, be remarked that contouring effects can occur in gradual luminance transitions, like skies, etc. in natural images. Contouring effects are not taken into account in the model. Therefore, usually a much higher number of quantization levels is used than the number of three bits that appears already to be sufficient for the example given here. In practice generally eight bits are used.

\subsection{Summary and conclusions}

In this chapter a survey has been given of the effect of various parameters on image quality. They cover areas of resolution, image size, viewing distance, luminance, contrast, gamma, noise, pixel density and luminance quantization. The effects have been analyzed with the aid of the SQRI metric given in the previous chapter. This metric appears to have a very good relation with subjectively perceived image quality. For the contrast sensitivity of the eye used in this metric, use has been made of the general expression given by Eq. (3.26) in Chapter 3. In most cases the calculated effect on image quality could be compared with published measurements. Measurements and calculations showed a very good agreement, also in situations where two parameters were varied simultaneously. 
For the application on special areas, like gamma, pixel density and luminance quantization, additional models had to be introduced. The results obtained with these models appeared also to be in good agreement with the measurement data.

\section{References}

Barten, P.G.J. (1987). The SQRI method: a new method for the evaluation of visible resolution on a display. Proceedings of the SID, 28, 253-262.

Barten, P.G.J. (1989a). The square root integral (SQRI): a new metric to describe the effect of various display parameters on perceived image quality. Human Vision, Visual Processing, and Digital Display I, Proc. SPIE, 1077, 73-82.

Barten, P.G.J. (1989b). Evaluation of CRT displays with the SQRI method. Proceedings of the SID, 30, 9-14.

Barten, P.G.J. (1990a). Evaluation of subjective image quality with the square-root integral method. Journal of the Optical Society of America A, 7, 2024-2031.

Barten, P.G.J. (1990b). Subjective image quality of high-definition television pictures. Proceedings of the SID, 31, 239-243.

Barten, P.G.J. (1991a). Evaluation of the effect of noise on subjective image quality. Human Vision, Visual Processing, and Digital Display II, Proc. SPIE, 1453, 2-15.

Barten, P.G.J. (1991b). Resolution of liquid-crystal displays. SID Digest, 22, 772 775.

Barten, P.G.J. (1991c). The effect of glass transmission on the subjective image quality of CRT pictures. Proceedings of the SID, 32, 285-288.

Barten, P.G.J. (1992). The SQRI as a measure for VDU image quality. SID Digest, 23, 867-870.

Barten, P.G.J. (1993). Effects of quantization and pixel structure on the image quality of color matrix displays. Journal of the SID, 1, 147-153.

Barten, P.G.J. (1996). Effect of gamma on subjective image quality. SID Digest, 27, 421-424.

Feng, Y., Östberg, O., and Lindström B. (1990). MTFA as a measure for computer display image quality. Displays, 11 , 186-192.

van Hateren, J.H. \& van der Schaaf, A. (1996). Temporal properties of natural scenes. Human Vision and Electronic Imaging, Proc. SPIE, 2657, 139-143.

Kayargadde, V. (1995). Feature extraction for image quality prediction. Ph. D. Thesis, Technical University of Eindhoven, Eindhoven, The Netherlands.

Kranz, J.H. \& Silverstein, L.D. (1990). Color matrix display image quality: the effects of luminance and spatial sampling. SID Digest, $21,29-32$. 
Mitsubayashi, T., Shimodaira, Y., Washio, H., Ikeda, H.; Muraoka, T., Mizushina, S. (1996). Gamma range of reproduced pictures having the quakity above acceptable limits. Proceedings Eurodisplay '96, 483-486.

Nijenhuis, M.R.M. (1993). Sampling and interpolation of static images: a perceptual view. Ph. D. Thesis, Technical University of Eindhoven, Eindhoven, The Netherlands.

Östberg, O. \& Feng, Y. (1991). The MTFA stick - an inexpensive measuring gauge for quick assessment of VDT display image resolution. SID Digest, 22, 779 780.

Roufs, J.A.J., Koselka, V.J.F., and van Tongeren, A.A.A.M. (1994). Global brightness contrast and the effect on perceptual image quality. Human Vision, Visual Processing, and Digital Display V, Proc. SPIE, 2179, 80-89.

Shimodaira, Y., Muraoka, T., Mizushina, S., Washio, H., Yamane, Y., Awane, K. (1995). Acceptable limits of gamma for a TFT-liquid crystal display on subjective evaluation of picture quality. IEEE Transactions on Consumer Electronics, 41, 550-554.

Silverstein, L.D., Kranz, J.H., Gomer, F.E., Yeh, Y.Y., and Monty, R.W. (1990). Effects of spatial sampling and luminance quantization on the image quality of color matrix displays. Journal of the Optical Society of America A, 7, 1955-1968.

Togerson, W.S. (1958). Theory and methods of scaling. John Wiley and Sons, New York.

Westerink, J.H.D.M. \& Roufs, J.A.J. (1989). Subjective image quality as a function of viewing distance, resolution, and picture size. SMPTE Journal, 98, 113-119.

van der Zee, E. \& Boesten, M.H.W.A. (1980). The influence of luminance and size on the image quality of complex scenes. IPO Annual Progress Report, 15, 69-75. 
196 


\section{Chapter 10}

\section{Epilogue}

In this study, a model has been given for the contrast sensitivity of the eye and its effect on perceived image quality. The model is based on the assumption that the contrast sensitivity is determined by internal noise in the visual system. In the different chapters, the model has been extended to various aspects of the visual system. In all cases, the model predictions have been compared with published measurement data. These measurements confirm the validity of the model.

For practical reasons, the model has been restricted to photopic vision. An extension of the model to scotopic vision would be useful. Although the contrast sensitivity of the eye and the perceived image quality is mainly determined by the achromatic properties of vision, an investigation of the possibilities for an extension to colour vision would also be useful.

Remarkable are some properties of the visual system that were met during the development of the model:

1. The constancy of the signal-to-noise ratio $k$ and the reason of its rather high value of 3 (See section 2.2).

2. The low value of the quantum efficiency $\eta$ of $3 \%$ (See section 3.4).

3. The limitation of the effective integration area of the eye by a maximum number of 15 cycles (See sections 2.4 and 4.4.2).

These properties should be investigated more deeply to get a better insight in the behavior of the visual system.

The constants used in the model are typical values that are largely based on contrast sensitivity measurements with young adult observers between 18 and 28 years of age. It would be interesting to investigate how these constants change with age from childhood to old age. Furthermore, it would be interesting to investigate if the effect of visual defects, like cataract, glaucoma, macular degeneration, etc. on contrast sensitivity can be described by changes of some of these constants. This might give a better insight in these defects

For future experiments with measurements of the contrast sensitivity function 
the following measures could be advised:

1. Using the psychometric for determining the modulation threshold. This gives the most accurate measurement results.

2. Applying also external noise. This gives extra information of the constants that play a role in the contrast sensitivity.

3. Using a constant field size and a constant viewing distance, instead of keeping the number of cycles constant by changing the field size and the viewing distance. By using a constant field size, other factors that could influence the results, like pupil size, the used area of the retina, etc. are constant.

For the given models a large number of assumptions had to be made about the biological structure of the retina and the functioning of retinal elements and nerve fibres. It would be useful, if these assumptions could be checked by biological and anatomical measurements. This would give a deeper insight in the visual system and could probably support the validity of the models. 


\section{Summary}

The observation of images by the eye is to a large amount determined by the sensitivity of the eye for luminance differences. This sensitivity is called contrast sensitivity and is defined by the reciprocal of the modulation of a sinusoidal luminance pattern that can just be observed. This modulation is called the modulation threshold.

In Chapter 2, it has been described how the modulation threshold can be determined in a well-defined way with the aid of the psychometric function, which represents the probability of detection as a function of the modulation. The modulation threshold is defined as the modulation for which the detection probability is $50 \%$. This modulation appears to be a fixed factor $k$ larger than the standard deviation of the probability distribution that forms the underlaying function of the psychometric function. In this study, it is assumed that this probability distribution is caused by internal noise in the visual system and that the standard deviation of it is equal to the average modulation of the noise. The factor $k$ can be considered as the signal-to-noise ratio and is about 3 . In this chapter also a principal analysis of the noise has been given with equations for the calculation of the modulation threshold from the data of this noise. For the use of these equations in the following chapters, it has been taken into account that the visual system is limited by maximum sizes of the spatial and temporal dimensions at the treatment of information.

In Chapter 3, the equations given in the previous chapter have been used for the presentation of a model for the contrast sensitivity of the eye based on internal noise in the visual system. This noise consists partly of photon noise and partly of neural noise. The contrast sensitivity is further also limited by the optical modulation transfer function of the eye and by the lateral inhibition. The first reduces the contrast sensitivity of the eye at high spatial frequencies, whereas the second reduces the contrast sensitivity at low spatial frequencies. The optical modulation transfer function is determined by the optical properties of the eye lens and by the mutual distance between the retinal elements and the lateral inhibition 
is determined by a spatial filtering of the information in the neural system. The so obtained model gives the contrast sensitivity of the eye as a function of the spatial frequency and as a function of the luminance and the field size. In the equation for this model, nine constants are used of which six can be considered as fixed and three can vary per subject or per experiment. For practical reasons, the use of the model is limited to photopic light levels. At the end of the chapter, a large number of published measurements of the contrast sensitivity function have been given in comparison with calculations obtained with the model.

In Chapter 4, the model given in the previous chapter, which is valid for the normally used central part of the retina, has been extended for use in the more outside located part of the retina. This part is important for the observation of objects outside the object on which the eye is directly concentrated. The extension has been obtained by varying the constants used in the model as a function of the retinal eccentricity. Various published measurements of the contrast sensitivity for different places of the retina were compared with calculations obtained with the model. The model was also used to calculate the density of on-centre $M$ ganglion cells from the measurements for different locations of the retina. The results were compared with data of biological measurements.

In Chapter 5, the spatial contrast sensitivity model given in Chapter 3 has been extended to the temporal domain. For the so obtained spatiotemporal model, it was assumed that the lateral inhibition signal undergoes also temporal filtering besides spatial filtering. With this model, the remarkable spatiotemporal behaviour of the visual system can be explained. A large number of published measurements of the contrast sensitivity as a function of the temporal frequency have been compared with calculations obtained with the model.

An important aspect of the temporal behaviour of the eye is the sensitivity for flicker. The flicker can, for instance, occur at images of television and computer systems, where flicker can be visible in an annoying way if the repetition frequency of the images is too low. With the aid of the given spatiotemporal model, the critical flicker frequency was calculated for television and computer images as a function of the luminance. By taking the interruption time of the image during the vertical retracing into account, a very good agreement was obtained with published measurements of the observation of flicker at these images.

The spatiotemporal contrast sensitivity model has also been used to calculate the temporal impulse response function of the eye. The so obtained impulse response function has a triphasic shape that differs from the twophasic shape obtained with other published models. This triphasic shape leads to a sharpening of temporal luminance variations in the observed image and improves in this way the sensitivity of the eye for these changes, just like the well-known Mach-band effect 
does for local luminance differences.

In Chapter 6, the equations given in Chapter 2 for the effect of the normal type of noise on the contrast sensitivity of the eye, have been extended to the effect of nonwhite noise. The normal type of noise is white noise, where the noise is equally distributed over the considered frequency range. At nonwhite noise, the distribution is not equal, or limited to a certain part of this range. The generalization of the equations is obtained by the assumption of a distribution function that describes the masking by one spatial frequency on the observation of another. The so calculated contrast sensitivity has been compared with measurements for situations with and without white and nonwhite noise.

In Chapter 7, a model has been given for contrast discrimination. At contrast discrimination, a difference has to be observed between two sinusoidally modulated signals with a small difference in modulation. The given model is based on the assumption that contrast discrimination can be considered as a special form of masking of one signal by the presence of another signal. With the equations for nonwhite noise given in the previous chapter, the masking can be expressed in the modulation of the signal used as reference signal. In the so obtained model an adaptation has been made for the nonlinearity of the eye at modulations above the threshold level. Various published measurements of contrast discrimination have been compared with calculations obtained with the model, where also the typical dipper shape of the measurements could be explained as a function of the modulation of the reference signal.

The contrast discrimination model has further been generalized by dividing the modulation threshold for contrast discrimination and the modulation of the reference signal by the modulation threshold for detection. This makes the results independent from spatial frequency, luminance and field size. The so obtained generalized model is important for the description of the behaviour of the visual system at modulations above the threshold level.

In Chapter 8, a measure has been given for the description of the perceived image quality. The quality of the image is for a large part determined by luminance modulations above the threshold level. With the aid of the generalized contrast discrimination model, it was derived that the perceived image quality is proportional with the square root of the normalized modulation. An image quality measure called SQRI (square-root integral) has been given, which is based on this principle. Various image quality measures have been compared on their functional suitability for the description of image quality. From this comparison, it appears, that the SQRI is most suitable for this purpose.

In Chapter 9, a survey has been given of the effect of different parameters 
on image quality. They concern resolution, image size, viewing distance, luminance, contrast, gamma, noise, pixel density and luminance quantization. These effects have been analyzed with the SQRI and have been compared with image quality measurements for the effect of these parameters. Also in cases where two parameters were varied simultaneously, the calculated image quality appears to show a very good correlation with the measured image quality,

The central idea behind all models given in this study is the assumption that the contrast sensitivity of the eye is limited by the presence of noise in the visual system. With this starting point and some additional assumptions, many aspects of the visual system could be explained. 


\section{Samenvatting}

De waarneming van beelden door het oog wordt in belangrijke mate bepaald door de gevoeligheid van het oog voor luminantieverschillen. Deze gevoeligheid wordt genoemd de contrastgevoeligheid en is gedefinieerd door de reciproque van de modulatie van een sinusvormig luminantiepatroon dat juist kan worden waargenomen. Deze modulatie wordt genoemd de drempelmodulatie.

In Hoofdstuk 2 is beschreven hoe de drempelmodulatie op een goed gedefinieerde wijze kan worden bepaald met behulp van de psychometrische functie, die de detectiewaarschijnlijkheid weergeeft als functie van de modulatie. De drempelmodulatie is gedefinieerd als de modulatie waarvoor de detectiewaarschijnlijkheid $50 \%$ is. Deze drempel blijkt een vaste factor $k$ groter te zijn dan de standaarddeviatie van de kansverdeling die de onderliggende functie vormt van de psychometrische functie. In deze studie is angenomen dat deze kansverdeling wordt veroorzaakt door inwendige ruis in het visuele systeem en dat de standaarddeviatie hiervan gelijk is aan de gemiddelde modulatie van de ruis. De factor $k$ kan worden beschouwd als de signaal- ruisverhouding en is ongeveer 3. In dit hoofdstuk is ook een principiële analyse van de ruis gegeven met formules voor de berekening van de drempelmodulatie uit gegevens van deze ruis. Voor het gebruik van deze formules in de volgende hoofdstukken is verder in aanmerking genomen dat het visuele systeem wordt beperkt door maximale afmetingen van de ruimtelijke en tijdelijke dimensies bij de bewerking van informatie.

In Hoofdstuk 3 zijn de in het vorige hoofdstuk gegeven formules gebruikt voor het geven van een model voor de contrastgevoeligheid van het oog gebaseerd op interne ruis in het visuele systeem. Deze ruis bestaat gedeeltelijk uit fotonruis en gedeeltelijk uit neurale ruis. De contrastgevoeligheid wordt verder ook nog beperkt door de optische modulatieoverdrachtsfunctie van het oog en de laterale inhibitie. De eerste vermindert de contrastgevoeligheid van het oog bij hoge spatiale frequenties, terwijl de tweede de contrastgevoeligheid vermindert bij lage spatiale frequenties. De optische modulatieoverdrachtsfunctie word bepaald door de optische eigenschappen van de ooglens en de onderlinge afstand tussen de netvliesele- 
menten en de laterale inhibitie wordt bepaald door een spatiale filtering van de informatie in het neurale systeem. Het zo verkregen model geeft de contrastgevoeligheid van het oog als functie van de spatiale frequentie en als functie van de luminantie en de grootte van het gezichtsveld. In de vergelijking voor dit model worden negen constanten gebruikt, waarvan er zes als vast kunnen worden beschouwd en er drie per individu of per experiment kunnen verschillen. Om praktische redenen is het gebruik van het model beperkt tot fotopische lichtniveaus. Aan het eind van het hoofdstuk zijn een groot aantal in de literatuur gepubliceerde metingen van de contrastgevoeligheidsfunctie gegeven in vergelijking met berekeningen verkregen met het model.

In Hoofdstuk 4 is het in het vorige hoofdstuk gegeven model, dat geldt voor het normaal gebruikte centrale deel van het netvlies, uitgebreid tot gebruik in het daarbuiten gelegen deel van het netvlies. Dit deel is van belang voor het waarnemen van objecten buiten die waarop het oog direct is geconcentreerd. De uitbreiding werd bereikt door een variatie van de in het model gebruikte constanten als functie van de excentriciteit op het netvlies. Verschillende in de literatuur gepubliceerde metingen van de contrastgevoeligheid voor verschillende plaatsen op het netvlies werden vergeleken met berekeningen verkregen met het model. Het model werd ook gebruikt om de dichtheid van on-centre $M$ ganglion cellen te berekenen uit de metingen voor verschillende plaatsen van het netvlies. De resultaten werden vergeleken met gegevens van biologische metingen.

In Hoofdstuk 5 is het in Hoofdstuk 3 gegeven model voor het spatiale domein uitgebreid tot het tijdsdomein. Voor het op deze wijze verkregen spatiotemporele model werd aangenomen dat het laterale inhibitiesignaal naast een spatiale filtering ook een temporele filtering ondergaat. Met dit model kan het merkwaardige spatiotemporele gedrag van het visuele systeem worden verklaard. Een groot aantal in de literatuur gepubliceerde metingen van de contrastgevoeligheid als functie van de temporele frequentie is vergeleken met berekeningen verkre-
gen met het model.

Een belangrijk aspect van het temporele gedrag van het oog is de gevoeligheid voor flikker. Flikker kan bijvoorbeeld optreden bij beelden van televisie- en computersystemen waarin flikker op een hinderlijke wijze waarneembaar is als de herhalingsfrequentie van de beelden te laag is. Met behulp van het gegeven spatiotemporele contrastgevoeligheidsmodel werd de kritische flikkerfrequentie berekend voor televisie- en computerbeelden als functie van de luminantie. Door het in aanmerking nemen van de onderbrekingstijd van het beeld tijdens de verticale terugslag werd een zeer goede overeenstemming verkregen met gepubliceerde metingen over de waarneembaarheid van flikker bij deze beelden.

Het spatiotemporele contrastgevoeligheidsmodel werd ook gebruikt om de 
temporele responsfunctie van het oog te berekenen. De zo verkregen impulsresponsfunctie heeft een driefasige verloop, dat verschilt van het tweefasige verloop dat met andere in de literatuur gepubliceerde modellen wordt verkregen. Dit driefasige verloop leidt tot een verscherping van temporele luminantieveranderingen in het waargenomen beeld en verbetert hierdoor de gevoeligheid van het oog voor deze veranderingen op een zelfde wijze als het bekende Mach-band effect dit doet voor plaatselijke luminantieverschillen.

In Hoofdstuk 6 zijn de in Hoofdstuk 2 gegeven formules voor het effect van het normale type ruis op de contrastgevoeligheid van het oog uitgebreid tot het effect van niet-witte ruis. Het normale type van ruis is witte ruis, waarbij de ruis gelijk is verdeeld over het beschouwde frequentiegebied. Bij niet-witte ruis is de verdeling ongelijk of beperkt tot een bepaald gedeelte van dit gebied. De generalisatie van de formules is verkregen door de aanname van een verdelingsfunctie die de maskering door één spatiale frequentie beschrijft op de waarneming van een andere. De op deze wijze berekende contrastgevoeligheid is vergeleken met metingen voor situaties met en zonder witte en niet-witte ruis.

In Hoofdstuk 7 is een model gegeven voor contrastdiscriminatie. Bij contrastdiscriminatie moet een verschil worden waargenomen tussen twee sinusvormig gemoduleerde luminantiesignalen met een klein verschil in modulatie. Het gegeven model is gebaseerd op de aanname dat contrastdiscriminatie kan worden beschouwd als een speciale vorm van maskering van één signaal door de aanwezigheid van een ander signaal. Met de in het vorige hoofdstuk gegeven formules voor nietwitte ruis kan deze maskering worden uitgedrukt in de modulatie van het signaal dat als referentiesignaal wordt gebruikt. In het zo verkregen model is ook een aanpassing gemaakt voor de nietlineariteit van het oog bij modulaties boven het drempelniveau. Verscheidene in de literatuur gepubliceerde metingen van contrastdiscriminatie zijn vergeleken met berekeningen verkregen met het model, waarbij ook de typische zeemeeuwvorm van de metingen als functie van de modulatie van het referentiesignaal kon worden verklaard.

Het contrastdiscriminatiemodel werd verder gegeneraliseerd door de modulatiedrempel voor de contrastdiscriminatie en de modulatie van het referentiesignaal te delen door de drempelmodulatie voor detectie. Dit maakt de resultaten onafhankelijk van spatiale frequentie, luminantie en beeldgrootte. Het zo verkregen gegeneraliseerde model is van belang voor de beschrijving van het gedrag van het visuele systeem bij modulaties boven de drempelwaarde.

In Hoofdstuk 8 is een maat gegeven voor de beschrijving van de waargenomen beeldkwaliteit. De kwaliteit van het beeld wordt in belangrijke mate bepaald door luminantiemodulaties boven het drempelniveau. Met behulp van het in het vorige hoofdstuk gegeven gegeneraliseerde contrastdiscriminatiemodel werd afge- 
leid dat de waargenomen beeldkwaliteit evenredig is met de wortel uit de genormaliseerde modulatie. Een beeldkwaliteitsmaat genaamde SQRI (square-root integral) is gegeven, die op dit principe is gebaseerd. Verschillende beeldkwaliteitsmaten zijn vergeleken op hun functionele geschiktheid voor het beschrijven van beeldkwaliteit. Hieruit blijkt dat de SQRI hiervoor het meest geschikt is.

In Hoofdstuk 9 is een overzicht gegeven van het effect van verschillende parameters op beeldkwaliteit. Zij betreffen resolutie, beeldgrootte, kijkafstand, luminantie, contrast, gamma, ruis, pixeldichtheid en kwantisering van de luminantie. Deze effecten zijn geanalyseerd met de SQRI en vergeleken met beeldkwaliteitsmetingen voor het effect van deze parameters. Ook in gevallen waarbij twee parameters gelijktijdig werden gevarieerd blijkt de berekende beeldkwaliteit een zeer goede correlatie te vertonen met de gemeten beeldkwaliteit,

De centrale gedachte achter alle in deze studie gegeven modellen is de veronderstelling dat de contrastgevoeligheid van het oog wordt begrensd door de aanwezigheid van ruis in het visuele systeem. Met dit uitgangspunt en enkele toegevoegde veronderstellingen konden veel eigenschappen van het visuele systeem worden verklaard. 


\section{Acknowledgement}

The author would like to express his thanks to Dr. A. Van Meeteren, Director of the Institute for Perception TNO, Soesterberg, for the fruitful and stimulating discussions at the start of this investigation, to Prof.Dr.Ir. J.A.J. Roufs, Prof.Dr.Ir. F.L. van Nes, and Dr.Ir. F.J.J. Blommaert of the Institute for Perception Research (IPO) of the Technical University of Eindhoven, and Prof.Dr. F.N. Hooge and Prof.Dr.Ing. H.J. Butterweck of the Department of Electrical Engineering of the same university for their useful advices for the manuscript, and to Dr. C.W. Tyler, Associate Director of the Smith-Kettlewell Eye Research Institute, San Francisco, for his useful comments on Chapter 5. 
208 


\section{Curriculum vitae}

Peter G.J. Barten was born in Amsterdam on December 1, 1927. In 1946 he obtained the Gymnasium $\beta$ certificate at the St. Ignatius college in Amsterdam. In 1953 he graduated in physics from the Technical University of Delft. After military service at the Royal Dutch Air Force, where he worked at the Department of Scientific Research, he joined the Research Laboratory of Philips in Eindhoven in 1956, where he was engaged in the development of storage tubes. In 1961 he transferred to the CRT Division of Philips, where he became in charge of the development of color picture tubes. At the end of 1987 he retired from Philips and started as an independent consultant, with a special emphasis on image quality. He developed the SQRI method for the evaluation of perceived image quality and a model for the spatiotemporal contrast sensitivity for the human eye. He is the author of about 30 technical papers and a chapter on Electron Optics in the TV \& Video Engineer's Reference Book. 
210 


\section{Stellingen}

behorende bij het proefschrift

Contrast sensitivity of the human eye and its effects on image quality van Peter Barten

1. De contrastgevoeligheid van het oog is grotendeels bepaald door interne ruis in het visuele systeem.

Dit proefschrift.

2. Door naast ruisvrije signalen ook signalen met ruis toe te passen bij metingen van de contrastgevoeligheid van het oog kan extra informatie worden verkregen over de interne ruis van het visuele systeem.

Dit proefschrift, hoofdstuk 3.

E.g. : Rovamo, J., Franssila, R., and Näsänen, R. (1992). Contrast sensitivity as a function of spatial frequency, viewing distance and eccentricity with and without spatial noise. Vision Research, 32, 631637.

3. De zichtbaarheid van flikker in televisie- en computerbeelden wordt behalve door de luminantie, de herhalingsfrequentie en de grootte van het beeld in belangrijke mate bepaald door de onderbrekingstijd tijdens de verticale terugslag. Deze wordt door Farrell e.a. ten onrechte niet meegenomen bij de bepaling van de flikkerdrempel.

Dit proefschrift, hoofdstuk 5 .

Farrell, J.E., Benson, B.E., and Haynie, C.R. (1987). Predicting flicker thresholds for visual displays. Proceedings of the SID, 28, 449-453.

4. De in de Amerikaanse ANSI standaard gebruikte MTFA (modulation transfer area) als maat voor de beeldkwaliteit is een onjuiste maat voor de werkelijk waargenomen beeldkwaliteit.

Dit proefschrift, hoofdstuk 8 .

ANSI/HFS 100, \$6.1.1.

5. De wet van de Vries-Rose voor de toename van de contrastgevoeligheid van het oog bij lage lichtniveaus met de wortel uit de luminantie zou naar zijn ontdekker, onze landgenoot de Vries, de wet van de Vries moeten worden 
genoemd. De bijdrage van Rose bestaat slechts uit de toepassing van deze wet enkele jaren later na kennisname van de publicatie van de Vries.

de Vries, H. (1943). The quantum character of light and its bearing upon threshold of vision, the differential sensitivity and visual acuity of the eye. Physica, 10, 553-564.

Rose, A (1948). The sensitivity performance of the human eye on an absolute scale. Joumal of the Optical Society of America, 38, 196-208.

6. Het effect van ruimteladingsafstoting op de grootte van een electronspot wordt niet verkregen door een kwadratische combinatie met die van andere effecten, zoals door Moss wordt aangenomen, maar door een lineaire optelling hierbij.

Moss, H. (1968). Narrow angle electron guns and cathode ray tubes, pp. 68 \& 89, Academic Press., New York \& London.

Barten, P.G.J. (1991). Electron optics in cathode ray tubes. TV \& Video Engineer's Reference Book, K.G. Jackson and G.B. Townsend, editors, Chapter 8, pp. 8/14-8/15, Butterworth-Heinemann, Oxford.

7. Het gebruik van de $50 \%$ breedte in plaats van de $5 \%$ breedte van de intensiteitsverdeling van een electronenspot in de Amerikaanse ANSI standaard voor beeldschermen, leidt tot een onjuist oordeel over de spotscherpte voor het algemene geval van een niet-Gaussische intensiteitsverdeling.

ANSI/HFS 100, $\$ 9.3$

Barten, P.G.J. (1986). Resolution of projection TV systems. SID Digest, 17, 455-458.

8. Door het uitschakelen van de natuurlijke selectie met behulp van de medische wetenschap breekt de mensheid bij zichzelf in enkele tientallen jaren af wat het evolutieproces in duizenden jaren heeft opgebouwd.

9. De uitdrukking "haaks staan op" wordt vaak ten onrechte gebruikt wanneer "lijnrecht tegenover" wordt bedoeld.

10. Het is met sommige theorieën als met ruis. $\mathrm{Zij}$ kunnen het zicht op de werkelijkheid belemmeren. 